ES/ER/TM-86/R1

\title{
Toxicological Benchmarks for Wildlife: 1994 Revision
}

\author{
D. M. Opresko ${ }^{1}$ \\ B. E. Sample ${ }^{2}$ \\ G. W. Suter ${ }^{2}$
}

Date Issued-September 1994

\begin{abstract}
Prepared by
Health Sciences Research Division ${ }^{1}$ and Environmental Sciences Division ${ }^{2}$

Oak Ridge National Laboratory under direction from the

Environmental Restoration Risk Assessment Council

Prepared for

United States Department of Energy

Office of Environmental Restoration and Waste Management

under budget and reporting code EW 20
\end{abstract}

OAK RIDGE NATIONAL LABORATORY

Oak Ridge, Tennessee 37831-6285

managed by

MARTIN MARIETTA ENERGY SYSTEMS, INC.

for the

U.S. DEPARTMENT OF ENERGY

under contract DE-AC05-84OR21400 
THIS PAGE INTENTIONALLY LEFT BLANK 


\section{DISCLAIMER}

This report was prepared as an account of work sponsored by an agency of the United States Government. Neither the United States Government nor any agency thereof, nor any of their employees, make any warranty, express or implied, or assumes any legal liability or responsibility for the accuracy, completeness, or usefulness of any information, apparatus, product, or process disclosed, or represents that its use would not infringe privately owned rights. Reference herein to any specific commercial product, process, or service by trade name, trademark, manufacturer, or otherwise does not necessarily constitute or imply its endorsement, recommendation, or favoring by the United States Government or any agency thereof. The views and opinions of authors expressed herein do not necessarily state or reflect those of the United States Government or any agency thereof. 


\section{DISCLAIMER}

Portions of this document may be illegible electronic image products. Images are produced from the best available original document. 


\section{CONTENTS}

EXECUTIVE SUMMARY $\ldots \ldots \ldots \ldots \ldots \ldots \ldots \ldots \ldots \ldots \ldots \ldots$

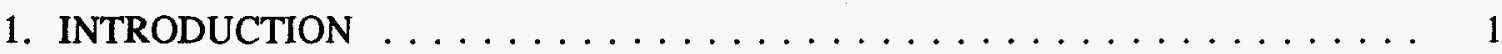

2. AVAILABILITY AND LIMITATIONS OF TOXICITY DATA $\ldots \ldots \ldots \ldots$

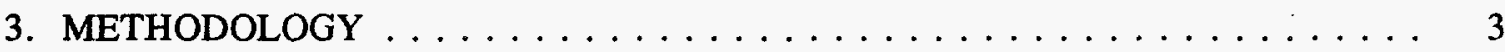

3.1 ESTIMATING NOAELS FOR WILDLIFE $\ldots \ldots \ldots \ldots \ldots \ldots \ldots$

3.2 DERIVING A CHRONIC NOAEL FROM OTHER ENDPOINTS $\ldots \ldots$

3.3 NOAEL EQUIVALENT CONCENTRATION IN FOOD . . . . . . . . . 7

3.4 NOAEL EQUIVALENT CONCENTRATION IN DRINKING WATER . . . 9

3.5 COMBINED FOOD AND WATER BENCHMARKS FOR AQUATIC FEEDING SPECIES $\ldots \ldots \ldots \ldots \ldots \ldots \ldots \ldots$

4. APPLICATION OF THE METHODOLOGY $\ldots \ldots \ldots \ldots \ldots \ldots \ldots \ldots \ldots$

4.1 INORGANIC TRIVALENT ARSENIC $\ldots \ldots \ldots \ldots \ldots \ldots \ldots$

4.1 .1 Toxicity to Wildlife . . . . . . . . . . . . . . 15

4.1 .2 Toxicity to Domestic Animals . . . . . . . . . . . . 15

4.1.3 Toxicity to Laboratory Animals (Rodents) . . . . . . . . . . . . 15

4.1.4 Extrapolations to Wildlife Species . . . . . . . . . . . 18

4.2 POLYCHLORINATED BIPHENYLS $\ldots \ldots \ldots \ldots \ldots \ldots \ldots$

4.2 .1 Toxicity to Wildlife . . . . . . . . . . . . 20

4.2 .2 Toxicity to Domestic Animals ............... 20

4.2 .3 Toxicity to Laboratory Animals . . . . . . . . . . . . 20

4.2 .4 Extrapolations to Wildlife Species . . . . . . . . . . 20

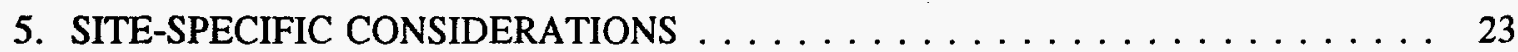

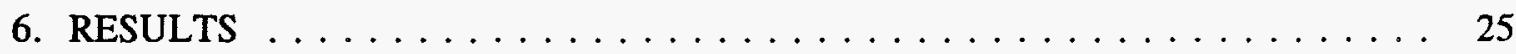

7. APPLICATION OF THE BENCHMARKS $\ldots \ldots \ldots \ldots \ldots \ldots \ldots \ldots \ldots \ldots$

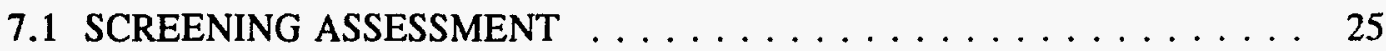

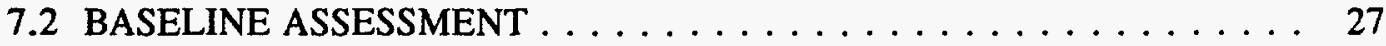

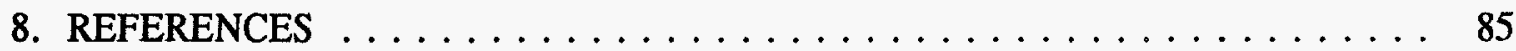

APPENDIX A Descriptions of Studies Used to Calculate Benchmarks . . . . . . . A-1

APPENDIX B Body Weights, Food and Water Consumption Rates for Selected Avian and Mammalian Wildlife Endpoint Species . . . . . . . . . . B-1

APPENDIX C Selected Toxicity Data for Avian and Mammalian Wildlife ....... C-1 


\section{TABLES}

Table 1. Reference values for mammalian species $\ldots \ldots \ldots \ldots \ldots \ldots \ldots$

Table 2. Aquatic food chain multiplying factors $\ldots \ldots \ldots \ldots \ldots \ldots \ldots \ldots \ldots$

Table 3. Octanol-water partition coefficients, bioconcentration factors, and bioaccumulation

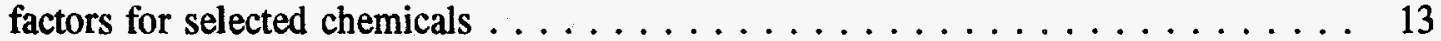

Table 4. Toxicity of trivalent arsenic compounds to wildlife $\ldots \ldots \ldots \ldots \ldots \ldots$

Table 6. Toxicity of trivalent arsenic compounds to laboratory animals $\ldots \ldots \ldots \ldots$

Table 7. Selected wildlife toxicity values for trivalent inorganic arsenic . . . . . . . . 19

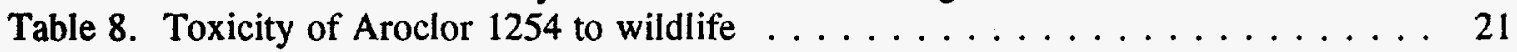

Table 9. Toxicity of Aroclor 1254 to laboratory animals . . . . . . . . . . . . 21

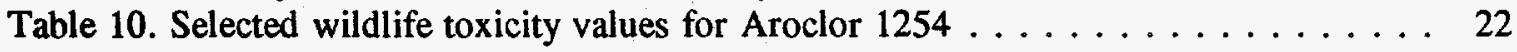

Table 11. Body size scaling factors . . . . . . . . . . . . . . . . . . . . 29

Table 12. Toxicological benchmarks for selected avian and mammalian wildlife species . . 30

Table 13. Use of benchmarks in a screening assessment . . . . . . . . . . . . . 84

Table 14. Use of benchmarks in a baseline assessment $\ldots \ldots \ldots \ldots \ldots$. . . . . . 84 


\section{ACRONYMS and ABBREVIATIONS}

$\begin{array}{ll}\text { BAF } & \text { Bioaccumulation Factor } \\ \text { BCF } & \text { Bioconcentration Factor } \\ \text { bw } & \text { Body Weight } \\ \text { COPC } & \text { Contaminant of Potential Concern } \\ \text { DOE } & \text { United States Department of Energy } \\ \text { EPA } & \text { United States Environmental Protection Agency } \\ \text { FCM } & \text { Food Chain Multiplier } \\ \text { FEL } & \text { Frank Effects Level } \\ \text { HQ } & \text { Hazard Quotient } \\ \text { LD } & \text { Lethal Dose to 50 percent of the population } \\ \text { LC } & \text { Lethal Concentration to 50 percent of the population } \\ \text { LOAEL } & \text { Lowest Observed Adverse Effects Level } \\ \text { NOAEL } & \text { No Observed Adverse Effects Level } \\ \text { Pot } & \text { Octanol/Water Partition Coefficient } \\ \text { PCB } & \text { Polychlorinated Biphenyl } \\ \text { RfD } & \text { Reference Dose } \\ \text { RTECS } & \text { Registry of Toxic Effects of Chemical Substances } \\ \text { TCDD } & \text { Tetrachlorodibenzodioxin } \\ \text { TCDF } & \text { Tetrachlorodibenzofuran } \\ \text { TWA } & \text { Time Weighted Average }\end{array}$





\section{ACKNOWLEDGEMENTS}

This manuscript has benefitted from the review comments of Tom Ashwood, Bob Young, Ruth Hull, and Bobette Nourse. We are also grateful for the assistance of Kit Lash in the preparation of this document. 
THIS PAGE INTENTIONALLY LEFT BLANK. 


\section{EXECUTIVE SUMMARY}

The process by which ecological risks of environmental contaminants are evaluated is twotiered. The first tier is a screening assessment where concentrations of contaminants in the environment are compared to toxicological benchmarks which represent concentrations of chemicals in environmental media (water, sediment, soil, food, etc.) that are presumed to be nonhazardous to the surrounding biota. The second tier is a baseline ecological risk assessment where toxicological benchmarks are one of several lines of evidence used to support or refute the presence of ecological effects.

The report presents toxicological benchmarks for assessment of effects of 76 chemicals on 8 representative mammalian wildlife species and 31 chemicals on 9 avian wildlife species. The chemicals are some of those that occur at United States Department of Energy waste sites; the wildlife species were chosen because they are widely distributed and provide a representative range of body sizes and diets. Further descriptions of the chosen wildlife species and chemicals are provided in the report. The benchmarks presented in this report represent values believed to be nonhazardous for the listed wildlife species. These benchmarks only consider contaminant exposure through oral ingestion of contaminated media; exposure through inhalation or direct dermal exposure are not considered in this report. 


\section{INTRODUCTION}

The process by which the ecological risks of environmental contaminants is evaluated is twotiered. In the first tier, a screening assessment is performed where concentrations of contaminants in the environment are compared to toxicological benchmarks. These benchmarks represent concentrations of chemicals in environmental media (water, sediment, soil, food, etc.) that are presumed to be nonhazardous to the biota. While exceedance of these benchmarks does not indicate any particular level or type of risk, concentrations below the benchmarks should not result in significant effects. In practice, when contaminant concentrations in food or water resources are less than these toxicological benchmarks, these contaminants may be excluded from further consideration. If, however, the concentration of a contaminant exceeds a benchmark, that contaminant should be retained as a contaminant of potential concern (COPC) and be subject to further investigation.

Toxicological benchmarks may also be used as part of a weight-of-evidence approach (Suter, 1993) in a baseline ecological risk assessment, the second tier in ecological risk assessment. Under this approach, toxicological benchmarks are one of several lines of evidence used to support or refute the presence of ecological effects. Other sources of evidence include media toxicity tests, surveys of biota (abundance and diversity), measures of contaminant body burdens, and biomarkers.

This report presents toxicological benchmarks for assessment of effects of 76 chemicals on 8 representative mammalian wildlife species (short-tailed shrew, little brown bat, meadow vole, white-footed mouse, cottontail rabbit, mink, red fox, and whitetail deer) and 31 chemicals on 9 avian wildlife species (American robin, American woodcock, wild turkey, belted kingfisher, great blue heron, barred owl, barn owl, Cooper's hawk, and red-tailed hawk) (scientific names are presented in Appendix B). These species were chosen because they are widely distributed and provide a representative range of body sizes and diets. The chemicals are some of those that occur at United States Department of Energy (DOE) waste sites. The benchmarks presented in this report represent values believed to be nonhazardous for the listed wildlife species. These benchmarks only consider contaminant exposure through oral ingestion of contaminated media. Exposure through inhalation or direct dermal exposure are not considered in this report.

\section{AVAILABILITY AND LIMITATIONS OF TOXICITY DATA}

Information on the toxicity of environmental contaminants to terrestrial wildlife can be obtained from several sources including the United States Environmental Protection Agency (EPA) Terrestrial Toxicity Data Base (TERRE-TOX, see Meyers and Schiller, 1986); U. S. Fish and Wildlife Service reports, EPA assessment and criteria documents, and Public Health Service toxicity profiles. In addition, many referred journals (e.g., Environmental Toxicology and Chemistry, Archives of Environmental Contamination and Toxicology, Journal of Wildlife Management, etc.) regularly publish studies concerning contaminant effects on wildlife. Selected data from these sources are presented in tabular form in Appendix C. Pesticides were excluded 
from this compilation except for those considered to be likely contaminants on DOE reservations, such as the persistent organochlorine compounds (e.g., Chlordane, DDT, Endrin, etc.). Most of the available information on the effects of environmental contaminants on wildlife pertains to agricultural pesticides and little to industrial and laboratory chemicals of concern to DOE. Furthermore, the toxicity data that are available are often limited to severe effects of acute exposures [e.g., concentration or dose levels causing $50 \%$ mortality to a test population $\left(\mathrm{LC}_{50}\right.$ and $\mathrm{LD}_{\mathrm{s0}_{0}}$ )]. Relatively few studies have determined safe exposure levels (no-observed-adverseeffect-levels, or NOAELs) for situations in which wildlife have been exposed over an entire lifetime or over several generations. [In this document, NOAEL refers to both dose (mg contaminant per $\mathrm{kg}$ animal body weight per day) and concentration (mg contaminant per $\mathrm{kg}$ of food or L of drinking water)]. Consequently, for nearly all wildlife species, a NOAEL for chronic exposures to a particular chemical must be estimated from toxicity studies of the same chemical conducted on a different species of wildlife or on domestic or laboratory animals or from less than ideal data (e.g., $L_{\text {so }}$ values). In many cases, the only available information is from studies on laboratory species (primarily rats and mice). These studies may be of short-term or subchronic duration and may only identify a lowest-observed-adverse-effect-level (LOAEL) and not a NOAEL. Estimating a NOAEL for a chronic exposure from such data can introduce varying levels of uncertainty into the calculation (see Subsect. 3.2); however, such laboratory studies represent a valuable resource whose use should be maximized.

Wildlife NOAELs estimated from data on laboratory animals must be evaluated carefully, bearing in mind the possible limitations of the data. Variations may exist among species in physiological or biochemical factors such as uptake, metabolism, and disposition, which can alter the potential toxicity of a contaminant to a particular species. Inbred laboratory strains may have an unusual sensitivity or resistance to the tested compound. Behavioral and ecological parameters (e.g., stress factors such as competition, seasonal changes in temperature or food availability, diseased states, or exposure to other contaminants) may make a wildlife species' sensitivity to an environmental contaminant different from that of a laboratory or domestic species.

Available studies on wildlife or laboratory species may not include evaluations of all significant endpoints for determining long-term effects on natural populations. Important data that may be lacking are potential effects on reproduction, development, and population dynamics following multigeneration exposures. In this report, endpoints such as reproductive and developmental toxicity, and reduced survival were used whenever possible; however, for some contaminants, limitations in the available data necessitated the use of endpoints such as organspecific toxic effects. It should be emphasized that in such cases the resulting benchmarks represent very conservative values whose relationship to potential population level effects is uncertain. These benchmarks will be recalculated if and when more appropriate toxicity data become available.

The fewer steps in the extrapolation process, the lower the uncertainty in estimating the wildlife NOAEL. For example, extrapolating from a NOAEL for an appropriate toxic endpoint (i.e., reproductive or population effects) for white laboratory mice to white-footed mice that are relatively closely related and of comparable body size would have a high level of reliability. Conversely, extrapolating from a LOAEL for organ-specific toxicity (e.g., liver or kidney damage) in laboratory mice to a non-rodent wildlife species such as mink or fox would have a low level of reliability in predicting population effects among these species. Because of the 
differences in avian and mammalian physiology and to reduce extrapolation uncertainty, studies performed on mammalian test species are used exclusively to estimate NOAELs for mammalian wildlife and studies performed on avian test species are used exclusively to estimate NOAELs for avian wildlife; interclass extrapolations were not performed.

In this report, benchmarks for mammalian species of wildlife have been estimated from studies conducted primarily on laboratory rodents, and benchmarks for avian species have been estimated from studies on domestic and wild birds. Very few experimental toxicity data are available for other groups of wildlife such as reptiles and amphibians, and it is not considered appropriate to apply benchmarks across different groups. Models for such wildlife extrapolations have not been developed as they have for aquatic biota (Suter, 1993).

\section{METHODOLOGY}

The general method used in this report is one based on EPA methodology for deriving human toxicity values (e.g., Reference Values, Reportable Quantities, and unit risks for carcinogenicity) from animal data (EPA, 1986a, 1986b, 1988b, 1989). In the method used herein experimentally derived NOAELs or LOAELs are used to estimate NOAELs for wildlife by adjusting the dose according to differences in body size. The concentrations of the contaminant in the wildlife species' food or drinking water that would be equivalent to the NOAEL are then estimated from the species' rate of food consumption and water intake. For wildlife species that feed primarily on aquatic organisms, a benchmark that combines exposure through both food and water is also calculated based on the potential of the contaminant to bioconcentrate and bioaccumulate through the food chain.

NOAELs and LOAELs for mammals and domestic and wild birds were obtained from the primary literature, EPA review documents, and secondary sources such as the Registry of Toxic Effects of Chemical Substances and the Integrated Risk Information System (IRIS). These studies are briefly described and the rationale for their use in deriving benchmarks is discussed in Appendix A. The selection of a particular study and a particular toxicity endpoint and the identification of NOAELs and LOAELs was based on our evaluation of the data. Emphasis was placed on those studies in which reproductive and developmental endpoints were considered (endpoints that may be directly related to potential population-level effects), multiple exposure levels were investigated, and the reported results were evaluated statistically to identify significant differences from control values. It is recognized that other interpretations of the same data may be possible and future research may provide more comprehensive data from which benchmarks

might be derived. Therefore, it is anticipated that the development of these screening benchmarks will be an ongoing process and, consequently, the values presented in this report are subject to change.

\subsection{ESTIMATING NOAELS FOR WILDLIFE}

NOAELs and LOAELs are daily dose levels normalized to the body weight of the test animals (e.g., milligrams of chemical per kilogram body weight per day). The presentation of 
toxicity data on a $\mathrm{mg} / \mathrm{kg} / \mathrm{day}$ basis allows comparisons across tests and across species with appropriate consideration for differences in body size. Studies have shown that numerous physiological functions such as metabolic rates, as well as responses to toxic chemicals, are a function of body size. Smaller animals have higher metabolic rates and are usually more resistant to toxic chemicals because of more rapid rates of detoxification. (However, this may not be the case if the toxic effects of the compound are produced primarily by a metabolite). It has been shown that the best measure of differences in body size is one based on body surface area which, for lack of direct measurements, can be expressed in terms of body weight (bw) raised to the $2 / 3$ power $\left(\mathrm{bw}^{2 / 3}\right)$ (EPA, 1980a). If the dose (d) itself has been calculated in terms of unit body weight (i.e., $\mathrm{mg} / \mathrm{kg}$ ), then the dose per unit body surface area (D) equates to:

$$
D=\frac{d x b w}{b w^{2 / 3}}=d x b w^{1 / 3}
$$

The assumption is that the dose per body surface area (Equation 1) for species "a" and "b" would be equivalent:

$$
d_{a} \times b w_{a}^{1 / 3}=d_{b} \times b w_{b}^{1 / 3}
$$

Therefore, knowing the body weights of two species and the dose $\left(d_{b}\right)$ producing a given effect in species " $b$," the dose $\left(d_{a}\right)$ producing the same effect in species " $a$ " can be determined:

$$
d_{a}=d_{b} \times \frac{b w_{b}^{1 / 3}}{b w_{a}^{1 / 3}}=d_{b} x\left(\frac{b w_{b}}{b w_{a}}\right)^{1 / 3}
$$

This is the methodology that EPA uses in carcinogenicity assessments and reportable quantity documents for adjusting from animal data to an equivalent human dose (EPA, 1985a, 1988b). The same approach has been proposed for use in extrapolating from one animal species to another. However, it should be noted that this method has not been applied to wildlife by the EPA and that wildlife toxicologists commonly scale dose to body weight without incorporating the exponential factor of $2 / 3$. The exponent has been retained for this report because no reason exists why different methods should be used to extrapolate from mice to humans and mice to foxes. The issue of appropriate scaling models for wildlife should be investigated.

For developing reference doses (RfDs), EPA uses a default factor of 0.1 to adjust an animal dose to an equivalent human dose. Using the body size scaling method outlined previously results in an adjustment factor of about 0.07 when deriving an equivalent human dose from data 
for mice (using the standard body weight of $0.03 \mathrm{~kg}$ for mice and $70 \mathrm{~kg}$ for humans) and a factor of about 0.17 when deriving an equivalent human dose from data for rats (standard body weight $0.35 \mathrm{~kg})$.

The ideal data set to use in the calculation would be the actual average body weights of the test animals used in the bioassay. When this information is not available, standard reference body weights for laboratory species can be used as indicated previously (EPA, 1985a, see Table 1). Body weight data for wildlife species are available from several secondary sources [i.e., the Mammalian Species series, published by the American Society of Mammalogists, Burt and Grosseneider, 1976; Dunning, 1984; Whitaker, 1980]. Often, only a range of adult body weight values is available for a species, in which case an average value must be estimated. A timeweighted average body weight for the entire life span of a species would be the most appropriate data set to use for chronic exposure situations; however, such data are usually not available. Body weight of a species can also vary geographically, as well as by sex. Sex-specific data may be needed depending on the toxicity endpoints used. Body weight data for the mammalian wildlife species considered in this report are given in Table 1.

Table 1. Reference values for mammalian species

\begin{tabular}{|c|c|c|c|c|c|}
\hline Species & $\begin{array}{l}\text { bw } \\
(\mathbf{k g})\end{array}$ & $\begin{array}{c}\text { Food Intake } \\
\text { (kg/day) }\end{array}$ & $\begin{array}{c}\text { Food factor" } \\
\quad f \\
\end{array}$ & $\begin{array}{c}\text { Water Intake } \\
\text { (L/day) })^{(199}\end{array}$ & $\begin{array}{c}\text { Water factor } \\
\omega \\
\end{array}$ \\
\hline rat & $0.35^{\circ}$ & $0.028^{d}$ & 0.08 & $0.046^{e}$ & 0.13 \\
\hline mouse & $0.03^{c}$ & $0.0055^{d}$ & 0.18 & $0.0075^{e}$ & 0.25 \\
\hline rabbit & $3.8^{\mathrm{c}}$ & $0.135^{d}$ & 0.034 & $0.268^{e}$ & 0.070 \\
\hline $\operatorname{dog}$ & $12.7^{\circ}$ & $0.301^{\mathrm{d}}$ & 0.024 & $0.652^{e}$ & 0.051 \\
\hline short-tailed shrew & $0.015^{\mathrm{f}}$ & $0.009^{f}$ & 0.6 & $0.0033^{f}$ & 0.22 \\
\hline meadow vole & $0.044^{f}$ & $0.005^{f}$ & 0.114 & $0.006^{8}$ & 0.136 \\
\hline white-footed mouse & $0.022^{f}$ & $0.0034^{8}$ & 0.155 & $0.0066^{f}$ & 0.3 \\
\hline cotton rat & 0.15 & $0.010^{\mathrm{h}}$ & 0.07 & $0.018^{\mathrm{g}}$ & 0.12 \\
\hline cottontail rabbit & $1.2^{f}$ & $0.237^{f}$ & 0.198 & $0.116^{8}$ & 0.013 \\
\hline $\operatorname{mink}$ & $1.0^{f}$ & $0.137^{f}$ & 0.137 & $0.099 \mathrm{~g}$ & 0.099 \\
\hline red fox & $4.5^{f}$ & $0.45^{f}$ & 0.1 & $0.38^{8}$ & 0.084 \\
\hline whitetail deer & $56.5^{r}$ & $1.74^{f}$ & 0.031 & $3.7^{8}$ & 0.065 \\
\hline
\end{tabular}

- The food factor is the daily food intake divided by the body weight.

- The water factor is the daily water intake divided by the body weight.

c EPA reference values (EPA, 1985a).

d Calculated using reference body weight and Equation 10.

- Calculated using reference body weight and Equation 21.

${ }^{f}$ see Appendix B for data source.

B Calculated according to Calder and Braun, 1983; see Equation 24.

' Calculated using Equation 14. 
If a NOAEL is available for the test species (NOAEL), then the equivalent NOAEL for a species of wildlife (NOAEL ${ }_{w}$ ) can be calculated by using the adjustment factor for differences in body size:

$$
N O A E L_{w}=N O A E L_{t}\left(\frac{b w_{t}}{b w_{w}}\right)^{1 / s}
$$

\subsection{DERIVING A CHRONIC NOAEL FROM OTHER ENDPOINTS}

In cases where a NOAEL for a specific chemical is not available for either wildlife or laboratory species, but a LOAEL has been determined experimentally, the NOAEL can be estimated by applying an uncertainty factor (UF) to the LOAEL. In the EPA methodology, the LOAEL can be reduced by a factor of up to 10 to derive the NOAEL.

$$
N O A E L=\frac{L O A E L}{\leq 10}
$$

Although a factor of 10 is usually used in the calculation, the true NOAEL may be only slightly lower than the experimental LOAEL, particularly if the observed effect is of low severity. A thorough analysis of the available data for the dose-response function may reveal whether a LOAEL to NOAEL uncertainty factor of $<10$ should be used. No data were found for any of the contaminants considered suggesting the use of a LOAEL-NOAEL adjustment factor of less than 10 .

If the only available data consist of a NOAEL (or a LOAEL) for a subchronic exposure, then the equivalent NOAEL or LOAEL for a chronic exposure can be estimated by applying a UF of $\leq 10$ :

$$
\text { chronic NOAEL }=\frac{\text { subchronic NOAEL }}{\leq 10}
$$

EPA has no clear guidance on the dividing line between a subchronic exposure and a chronic exposure. For studies on laboratory rodents, EPA generally accepts a 90-day exposure duration as a standard for a subchronic exposure. In the guidance for the proposed Great Lakes Water Quality Criteria, EPA (1993d) indicates that a chronic exposure would be equivalent to at least $50 \%$ of a species lifespan. Since most of the NOAELS and LOAELS available for calculated benchmarks for mammalian wildlife are from studies on laboratory rodents (with lifespans of approximately 2 years), we have selected 1 year as the minimum required exposure duration for 
a chronic exposure (approximately one-half of the lifespan). There is little information concerning the lifespans of birds used in toxicity tests and little standardization of study duration for avian toxicity tests. In addition, few long-term, multigeneration avian toxicity tests have been performed. Therefore avian studies where exposure duration was 10 weeks or less were considered to be subchronic and those where the exposure duration was greater than 10 weeks were considered chronic studies.

In addition to duration of exposure, the time when contaminant exposure occurs is critical. Reproduction is a particularly sensitive lifestage due to the stressed condition of the adults and the rapid growth and differentiation occurring within the embryo. For many species, contaminant exposure of a few days to as little as a few hours during gestation and embryo development may produce severe adverse effects. Because these benchmarks are intended to evaluate the potential for adverse effects on wildlife populations and impaired reproduction is likely to affect populations, contaminant exposures that are less than one year or 10 weeks but occur during reproduction were considered to represent chronic exposures.

If the available data are limited to acute toxicity endpoints (FEL, frank-effects level) or to exposure levels associated with lethal effects $\left(L D_{s 0} s\right)$, the estimation of NOAELs for chronic exposures are likely to have a wide margin of error because no standardized mathematical correlation exists between FEL or $\mathrm{LD}_{s 0}$ values and NOAELs that can routinely be applied to all chemicals (i.e., exposure levels associated with NOAELs may range from $1 / 10$ to $1 / 10,000$ of the acutely toxic dose, depending on the chemical and species). However, if both an $\mathrm{LD}_{50}$ and a NOAEL have been determined for a related chemical $a$, then this ratio could be used to estimate a NOAEL $L_{w}$ using the $\left(\mathrm{LD}_{50}\right)_{\mathrm{w}}$ for the compound of interest.

$$
N O A E L_{w}=\left(L D_{50}\right)_{w} \frac{N O A E L_{a}}{\left(L D_{50}\right)_{a}}
$$

\subsection{NOAEL EQUIVALENT CONCENTRATION IN FOOD}

The dietary level or concentration in food $\left(\mathrm{C}_{\mathrm{f}}\right.$, in $\mathrm{mg} / \mathrm{kg}$ food $)$ of a contaminant that would result in a dose equivalent to the NOAEL (assuming no other exposure through other environmental media) can be calculated from the food factor $f$ :

$$
C_{f}=\frac{N O A E L_{w}}{f}
$$


The food factor, $f$, is the amount of food consumed ( $\mathrm{F}$, in $\mathrm{g} /$ day or $\mathrm{kg} /$ day) per unit body weight (bw, in $\mathrm{g}$ or $\mathrm{kg}$ ):

$$
f=\frac{F}{b w}
$$

In the absence of empirical data, rates of food consumption ( $F$, in $\mathrm{kg} / \mathrm{day}$ ) for laboratory mammals can be estimated from allometric regression models based on body weight (in $\mathrm{kg}$ ) (EPA, 1988a):

$$
\begin{gathered}
F=0.056(b w)^{0.6611} \quad \text { (laboratory mammals) } \\
F=0.054(b w)^{0.9451} \quad(\text { moist diet }) \\
F=0.049(b w)^{0.6087} \quad(\text { dry diet })
\end{gathered}
$$

In the absence of specific information on the body weights of the test animals, EPA (1985a) uses default values (see Table 1). In this report, $F$ was estimated using Equation 10 and the default body weights. Reference body weights for particular strains of laboratory animals and for specific age groups corresponding to subchronic or chronic exposures are available (EPA, 1988a), and these can also be used in the equations. Default values for food consumption and food factors for common laboratory species (rats, mice, dogs, rabbits, etc.) have also been used by EPA (1988b) for estimating equivalent dose levels for laboratory studies in which the exposure is reported only as a dietary concentration. Generally, the rates of food consumption for laboratory species, as derived from Equations 10-12, are higher then the EPA default values.

Food consumption rates are available for some species of wildlife (EPA, 1993a, 1993b Table 1). In the absence of experimental data, $F$ values (g/day) can be estimated from allometric regression models based on metabolic rate and expressed in terms of body weight ( $g$ ) (Nagy, 1987):

$$
\begin{gathered}
F=0.235(b w)^{0.822} \quad \text { (placental mammals) } \\
F=0.621(b w)^{0.564} \quad \text { (rodents) } \\
F=0.577(b w)^{0.727} \quad \text { (herbivores) }
\end{gathered}
$$




$$
\begin{gathered}
F=0.492(b w)^{0.673} \quad(\text { marsupials) } \\
F=0.648(b w)^{0.651} \quad(\text { birds) } \\
F=0.398(b w)^{0.850} \quad \text { (passerine birds) }
\end{gathered}
$$

\subsection{NOAEL EQUIVALENT CONCENTRATION IN DRINKING WATER}

The concentration of the contaminant in the drinking water of an animal $\left(\mathrm{C}_{w}\right.$, in $\left.\mathrm{mg} / \mathrm{L}\right)$ resulting in a dose equivalent to a $\mathrm{NOAEL}_{w}$ can be calculated from the daily water consumption rate ( $\mathrm{W}$, in $\mathrm{L} /$ day) and the average body weight $\left(\mathrm{bw}_{\mathrm{w}}\right)$ for the species:

$$
C_{w}=\frac{N O A E L_{w} \times b w_{w}}{W}
$$

If known, the water factor $\omega$ (= the rate of water consumption per unit body weight $(\mathrm{W} / \mathrm{bw})$ can be used in a manner identical to that for the food factor.

$$
C_{w}=\frac{N O A E L_{w}}{\omega}
$$

If empirical data are not available, $W$ (in L/day) can be estimated from allometric regression models based on body weight (in $\mathrm{kg}$ ) (EPA, 1988a):

$$
\begin{aligned}
& W=0.10(b w)^{0.7377} \quad \text { (laboratory mammals) } \\
& W=0.009(b w)^{1.2044} \text { (mammals, moist diet) } \\
& W=0.093(b w)^{0.7584} \text { (mammals, dry diet) }
\end{aligned}
$$


In the absence of specific information on the body weights of the test animals, EPA (1985a) uses default values (see Table 1). In this report, W was estimated using Equation 21 and the default body weights. Reference body weights for particular strains of laboratory animals and for specific age groups corresponding to subchronic or chronic exposures are available (EPA, 1988a), and these can also be used in the equations. Default values for water consumption and $\omega$ for common laboratory species have been used by EPA (1988b) for estimating equivalent dose levels for laboratory studies in which the exposure was given only as a concentration in the animals' drinking water. Generally, the rates of water consumption for laboratory species, as derived from Equations 21-23, are higher then the EPA default values.

Water consumption rates are available for some species of mammalian wildlife (Table 1). Water consumption rates (in L/day) can also be estimated from allometric regression models based on body weight (in $\mathrm{kg}$ ) (Calder and Braun, 1983):

$$
W=0.099(b w)^{0.90}
$$

A similar model has also been developed for birds (Calder and Braun, 1983):

$$
W=0.059(b w)^{0.67}
$$

\subsection{COMBINED FOOD AND WATER BENCHMARKS FOR AQUATIC FEEDING SPECIES}

If a wildlife species (such as mink, belted kingfisher, or great blue heron) feeds primarily on aquatic organisms and the concentration of the contaminant in the food is proportional to the concentration in the water, then the food consumption rate $(\mathrm{F}$, in $\mathrm{kg} /$ day) and the aquatic life bioaccumulation factor can be used to derive a $\mathrm{C}_{\mathrm{w}}$ value that incorporates both water and food consumption (EPA, 1993c, 1993d, 1993e):

$$
C_{w}=\frac{N O A E L_{w} \times b w_{w}}{W+(F \times B A F)}
$$

The BAF is the ratio of the concentration of a contaminant in tissue $(\mathrm{mg} / \mathrm{kg}$ ) to its concentration in water $(\mathrm{mg} / \mathrm{L})$, where both the organism and its prey are exposed, and is expressed as L/kg. Bioaccumulation factors may be predicted by multiplying the bioconcentration factor for the contaminant $[\mathrm{BCF}$, ratio of concentration in food to concentration in water; i.e., $(\mathrm{mg} / \mathrm{kg}) /(\mathrm{mg} / \mathrm{L})=\mathrm{L} / \mathrm{kg}$ ] by the appropriate food chain multiplying factor (FCM) (see Table 2).

For most inorganic compounds, BCFs and BAFs are assumed to equal; however, an FCM may be applicable for some metals if the organometallic form biomagnifies (EPA, 1993c). 
11

Table 2. Aquatic food chain multiplying factors ${ }^{2}$

\begin{tabular}{|c|c|c|c|}
\hline \multirow[b]{2}{*}{$\log P_{\propto c t}$} & \multicolumn{3}{|c|}{ Prey Trophic Level } \\
\hline & 2 & 3 & 4 \\
\hline$\leq 3.9$ & 1.0 & 1.0 & 1.0 \\
\hline 4.0 & 1.1 & 1.0 & 1.0 \\
\hline 4.1 & 1.1 & 1.1 & 1.1 \\
\hline 4.2 & 1.1 & 1.1 & 1.1 \\
\hline 4.3 & 1.1 & 1.1 & 1.1 \\
\hline 4.4 & 1.2 & 1.1 & 1.1 \\
\hline 4.5 & 1.2 & 1.2 & 1.2 \\
\hline 4.6 & 1.2 & 1.3 & 1.3 \\
\hline 4.7 & 1.3 & 1.4 & 1.4 \\
\hline 4.8 & 1.4 & 1.5 & 1.6 \\
\hline 4.9 & 1.5 & 1.8 & 2.0 \\
\hline 5.0 & 1.6 & 2.1 & 2.6 \\
\hline 5.1 & 1.7 & 2.5 & 3.2 \\
\hline 5.2 & 1.9 & 3.0 & 4.3 \\
\hline 5.3 & 2.2 & 3.7 & 5.8 \\
\hline 5.4 & 2.4 & 4.6 & 8.0 \\
\hline 5.5 & 2.8 & 5.9 & 11.0 \\
\hline 5.6 & 3.3 & 7.5 & 16.0 \\
\hline 5.7 & 3.9 & 9.8 & 23.0 \\
\hline 5.8 & 4.6 & 13.0 & 33.0 \\
\hline 5.9 & 5.6 & 17.0 & 47.0 \\
\hline 6.0 & 6.8 & 21.0 & 67.0 \\
\hline 6.1 & 8.2 & 25.0 & 75.0 \\
\hline 6.2 & 10.0 & 29.0 & 84.0 \\
\hline 6.3 & 13.0 & 34.0 & 92.0 \\
\hline 6.4 & 15.0 & 39.0 & 98.0 \\
\hline
\end{tabular}


Table 2. (continued)

\begin{tabular}{|c|c|c|c|}
\hline \multirow[b]{2}{*}{$\log P_{o c t}$} & \multicolumn{3}{|c|}{ Prey Trophic Level } \\
\hline & 2 & 3 & 4 \\
\hline$\leq 3.9$ & 1.0 & 1.0 & 1.0 \\
\hline 6.5 & 19.0 & 45.0 & 100.0 \\
\hline$>6.5$ & $\left({ }^{c}\right)$ & $(\mathbf{c})$ & $\left(c^{c}\right)$ \\
\hline
\end{tabular}

'From U.S. EPA 1993c.

Trophic level: $\mathbf{2}=$ zooplankton; 3 = small fish; $\mathbf{4}=$ piscivorous fish, including top predators.

'For chemicals with $\log P_{\text {oet }}>6.5$, FCM can range from 0.1-100. Such chemicals should be evaluated individually. Without chemical-specific data, an FCM of 1.0 should be used

(EPA 1993c).

In cases where the BCF for a particular compound is not available, it can be estimated from the octanol-water partition coefficient of the compound by the following relationship (Lyman et al., 1982):

$$
\log B C F=0.76 \log P_{\text {oct }}-0.23
$$

The BCF can also be estimated from the water solubility of a compound by the following regression equation (Lyman et al., 1982):

$$
\log B C F=2.791-0.564 \log W S
$$

where WS is the water solubility in $\mathrm{mg} / \mathrm{L}$ water.

Log $P_{\text {oct }}$ values, reported or calculated BCF values, and estimated BAF values for chemicals for which benchmarks have been derived are included on Table 3. Reported BCFs represent the maximum value listed for fish. A FCM of 1 was applied to all reported BCFs for inorganic compounds (EPA, 1993c). Because all wildlife (mink, belted kingfisher, great blue heron), for which combined food and water benchmarks were calculated, consume small fish, the trophic level 3 FCM appropriate for the $\log \mathrm{P}_{\text {oct }}$ of the chemical was applied to all calculated BCFs. 
Table 3. Octanol-water partition coefficients, bioconcentration factors, and bioaccumulation factors for selected chemicals

\begin{tabular}{|c|c|c|c|c|c|}
\hline Chemical and Form & $\log P_{\text {ect }}$ & BCF & $\begin{array}{c}\text { Trophic } \\
\text { Level } 3 \\
\text { FCM }\end{array}$ & $\begin{array}{c}\text { Trophic } \\
\text { Level } 3 \\
\text { BAF }\end{array}$ & Source \\
\hline Acetone & -0.24 & $0.39^{2}$ & 1.0 & 0.39 & USAF 1989 \\
\hline Aluminum & & 231 & 1.0 & 231.00 & EPA $1988 c$ \\
\hline Antimony & & 1 & 1.0 & 1.00 & EPA $1980 b$ \\
\hline Aroclor 1016 & 5.6 & $10616.9^{\circ}$ & 7.5 & 79627.17 & ATSDR 1989 \\
\hline Aroclor 1242 & 5.6 & $10616.9^{*}$ & 7.5 & 79627.17 & ATSDR 1989 \\
\hline Aroclor 1248 & 6.2 & $30338.9^{\mathrm{a}}$ & 29.0 & 879828.44 & ATSDR 1989 \\
\hline Aroclor 1254 & 6.5 & $51286.1^{*}$ & 45.0 & 2307876.23 & ATSDR 1989 \\
\hline Arsenic (arsenite) & & 17.00 & 1.0 & 17.00 & EPA 1985g \\
\hline Benzene & 2.13 & $24.48^{\circ}$ & 1.0 & 24.48 & EPA 1992 \\
\hline BHC-mixed isomers & 5.31 & $6391.46^{\circ}$ & 3.7 & 23648.40 & EPA 1992 \\
\hline Benzo(a)pyrene & 6.1 & $25468.3^{2}$ & 25.0 & 636707.56 & EPA 1992 \\
\hline Beryllium & & 19.00 & 1.0 & 19.00 & EPA $1980 c$ \\
\hline Bis(2-ethylhexyl)phthalate & 5.11 & $4504.0^{\circ}$ & 2.5 & 11260.04 & EPA 1992 \\
\hline Cadmium & & 12400.00 & 1.0 & 12400.00 & EPA $1985 f$ \\
\hline Carbon Tetrachloride & 2.83 & $83.33^{\circ}$ & 1.0 & 83.33 & EPA 1992 \\
\hline Chlordane & 5.54 & $9558.73^{\circ}$ & 5.9 & 56396.48 & EPA 1992 \\
\hline Chloroform & 1.97 & $18.5^{*}$ & 1.0 & 18.50 & EPA 1992 \\
\hline Chromium $(\mathrm{Cr}+6)$ & & 3.00 & 1.0 & 3.00 & EPA 1985d \\
\hline Copper & & 290.00 & 1.0 & 290.00 & EPA 1985e \\
\hline o-Cresol & 1.95 & $17.86^{2}$ & 1.0 & 17.86 & EPA 1992 \\
\hline Cyanide & & 0.00 & 1.0 & 0.00 & EPA $1985 c$ \\
\hline DDT (and metabolites) & 6.36 & $40142.1^{3}$ & 39.0 & 1565541.58 & EPA 1992 \\
\hline 1,2-Dichloroethane & 1.48 & $7.85^{*}$ & 1.0 & 7.85 & EPA 1992 \\
\hline 1,1-Dichloroethylene & 2.13 & $24.48^{\circ}$ & 1.0 & 24.48 & EPA 1992 \\
\hline 1,2-Dichloroethylene & 1.86 & $15.26^{\circ}$ & 1.0 & 15.26 & EPA 1992 \\
\hline Dieldrin & 4.56 & $1720.28^{2}$ & 1.3 & 2236.37 & EPA 1992 \\
\hline Diethylphthalate & 2.47 & $44.38^{\circ}$ & 1.0 & 44.38 & EPA 1992 \\
\hline Di-n-butyl phthalate & 4.13 & $810.59^{\prime \prime}$ & 1.1 & 891.65 & EPA 1992 \\
\hline 1,4-Dioxane & -0.27 & $0.37^{\circ}$ & 1.0 & 0.37 & EPA 1992 \\
\hline Endrin & 4.56 & $1720.28^{*}$ & 1.3 & 2236.37 & EPA 1992 \\
\hline Ethanol & -0.31 & $0.34^{2}$ & 1.0 & 0.34 & EPA 1992 \\
\hline Formaldehyde & 0.35 & $1.09^{\mathrm{a}}$ & 1.0 & 1.09 & EPA 1992 \\
\hline
\end{tabular}


14

Table 3. (continued)

\begin{tabular}{|c|c|c|c|c|c|}
\hline Chemical and Form & $\log P_{\text {eet }}$ & BCF & $\begin{array}{c}\text { Trophic } \\
\text { Level } 3 \\
\text { FCM }\end{array}$ & $\begin{array}{c}\text { Trophic } \\
\text { Level } 3 \\
\text { BAF }\end{array}$ & Soturce \\
\hline Acetone & -0.24 & $0.39^{2}$ & 1.0 & 0.39 & USAF 1989 \\
\hline Heptachlor & 4.27 & $1035.62^{\circ}$ & 1.1 & 1139.18 & EPA 1992 \\
\hline Lead & & 45.00 & 1.0 & 45.00 & EPA $1985 b$ \\
\hline Lindane (Gamma-BHC) & 3.72 & $395.55^{\circ}$ & 1.0 & 395.55 & EPA 1992 \\
\hline Mercury (Methyl Mercury Chloride) & & & & 60000.00 & EPA $1993 \mathrm{e}$ \\
\hline Methanol & -0.77 & $0.15=$ & 1.0 & 0.15 & EPA 1992 \\
\hline Methylene Chloride & 1.25 & $5.25^{\circ}$ & 1.0 & 5.25 & EPA 1992 \\
\hline Methyl Ethyl Ketone & 0.29 & $0.98^{\circ}$ & 1.0 & 0.98 & EPA 1992 \\
\hline 4-Methyl 2-Pentanone & 1.19 & $4.72^{n}$ & 1.0 & 4.72 & EPA 1992 \\
\hline Nickel & & 106.00 & 1.0 & & EPA $1986 f$ \\
\hline Pentachloronitrobenzene & 4.64 & $1978.79^{\mathrm{s}}$ & 1.3 & 2572.43 & EPA 1992 \\
\hline Selenium & & & & 2600.00 & $\begin{array}{c}\text { Peterson and Nebeker } \\
1992\end{array}$ \\
\hline 2,3,7,8-Tetrachloro Dibenzodioxin & 6.8 & $86696.2^{2}$ & 1.0 & 86696.19 & EPA 1992 \\
\hline 1,1,2,2-Tetrachloroethylene & 3.4 & $225.94^{\circ}$ & 1.0 & 225.94 & EPA 1992 \\
\hline Thallium & & 34.00 & 1.0 & 34.00 & EPA 1980d \\
\hline Toluene & 2.73 & $69.95^{a}$ & 1.0 & 69.95 & EPA 1992 \\
\hline Toxaphene & 4.82 & $2711.44^{\mathrm{a}}$ & 1.5 & 4067.16 & EPA 1992 \\
\hline 1,1,1-Trichloroethane & 2.49 & $45.96^{\circ}$ & 1.0 & 45.96 & EPA 1992 \\
\hline Trichloroethylene & 2.42 & $40.66^{\circ}$ & 1.0 & 40.66 & EPA 1992 \\
\hline Vinyl Chloride & 1.36 & $6.36^{\circ}$ & 1.0 & 6.36 & EPA 1992 \\
\hline Xylene (mixed isomers) & 3.2 & $159.22^{x}$ & 1.0 & 159.22 & EPA 1992 \\
\hline Zinc & & 966.00 & 1 & 966.00 & EPA 1987 \\
\hline
\end{tabular}

- Values estimated using Equation 27

\section{APPLICATION OF THE METHODOLOGY}

Two examples will be given illustrating the application of the methodology for deriving NOAELs and screening benchmarks. In one example (inorganic trivalent arsenic), the estimated values were derived primarily from data on laboratory species. In the second example (Aroclor 1254, a polychlorinated biphenyl), experimental data were available for two species of mammalian wildlife. While the examples focus on mammals, derivation of NOAELs and screening benchmarks for birds is performed in an identical manner. 


\subsection{INORGANIC TRIVALENT ARSENIC}

The toxicity of inorganic compounds containing arsenic depends on the valence or oxidation state of the arsenic as well as on the physical and chemical properties of the compound in which it occurs. Trivalent $\left(\mathrm{As}^{+3}\right)$ compounds such as arsenic trioxide $\left(\mathrm{As}_{2} \mathrm{O}_{3}\right)$, arsenic trisulfide $\left(\mathrm{As}_{2} \mathrm{~S}_{3}\right)$, and sodium arsenite $\left(\mathrm{NaAsO}_{2}\right)$, are generally more toxic than pentavalent $\left(\mathrm{As}^{+5}\right)$ compounds such as arsenic pentoxide $\left(\mathrm{As}_{2} \mathrm{O}_{5}\right)$, sodium arsenate $\left(\mathrm{Na}_{2} \mathrm{HAsO}_{4}\right)$, and calcium arsenate $\left[\mathrm{Ca}_{3}\left(\mathrm{AsO}_{4}\right)_{2}\right]$. The relative toxicity of the trivalent and pentavalent forms may also be affected by factors such as water solubility; the more toxic compounds are generally more water soluble. In this analysis, the effects of the trivalent form of arsenic in water soluble inorganic compounds will be evaluated. In many cases, only total arsenic concentrations are reported so the assessor must conservatively assume that it is all trivalent.

\subsubsection{Toxicity to Wildlife}

The only wildlife toxicity information available for trivalent inorganic arsenic compounds pertains to acute exposures (Table 4; the values listed are those reported in the literature except where noted).

For whitetail deer, the estimated lethal dose is $34 \mathrm{mg}$ sodium arsenite $/ \mathrm{kg}$ or $19.5 \mathrm{mg} \mathrm{As} / \mathrm{kg}$ (NAS, 1977). For birds, estimated $L_{50}$ values for sodium arsenite range from 47.6 to $386 \mathrm{mg} / \mathrm{kg}$ body weight. Median lethal ity was also reported at a dietary level of $500 \mathrm{mg} / \mathrm{kg}$ food for mallard ducks. No information was found in the available literature regarding chronic toxicity or reproductive or developmental effects.

\subsubsection{Toxicity to Domestic Animals}

The toxicity of inorganic trivalent arsenic to domestic animals is summarized in Table 5 (the values listed are those given in the source). For assessment purposes, the most useful study is the one identifying a dietary NOAEL of $50 \mathrm{ppm}$ As in dogs following a 2 year exposure to sodium arsenite. This dietary concentration was estimated to be equivalent to $1.2 \mathrm{mg} / \mathrm{kg} \mathrm{bw} /$ day.

\subsubsection{Toxicity to Laboratory Animals (Rodents)}

Selected acute and chronic toxicity data for trivalent arsenic in rats and mice are summarized in Table 6 (dietary or drinking water concentrations were converted to daily dose levels using reference body weights and Equations 8 and 20). For assessment purposes, the studies of Byron et al. (1967) and that of Schroeder and Mitchener (1971) provide the most useful data. In the study of Bryon et al. (1967), a dietary concentration of $62.5 \mathrm{ppm}$ As for 2 years caused no adverse effects in rats other than a slight reduction in growth of females. This dietary level, which can be considered a NOAEL, is equivalent to a daily dose of $5 \mathrm{mg} \mathrm{As} / \mathrm{kg}$ bw/day. In the Schroeder and Mitchener (1971) study, a concentration of $5 \mathrm{mg} \mathrm{As} / \mathrm{L}$ in the drinking water of mice over three generations was associated with a decrease in litter size and therefore is considered a potential population level LOAEL. The equivalent dose was estimated to be $1.26 \mathrm{mg} / \mathrm{kg}$ bw/day; therefore, using Equation 5, the NOAEL is estimated to be $0.126 \mathrm{mg} / \mathrm{kg}$ bw/day. 
Table 4. Toxicity of trivalent arsenic compounds to wildlife

\begin{tabular}{|c|c|c|c|c|c|}
\hline Species & Chemical & $\begin{array}{l}\text { Conc. in Diet } \\
\text { (mg/kg food) }\end{array}$ & $\begin{array}{c}\text { Dose } \\
\text { (mg/kg) }\end{array}$ & Effect & Reference \\
\hline $\begin{array}{l}\text { Whitetail deer } \\
\text { (Odocollews virginianus) }\end{array}$ & $\begin{array}{l}\text { sodium } \\
\text { arsenite }\end{array}$ & NR & 34 & Lethal dose & NAS, 1977 \\
\hline \multirow[t]{2}{*}{$\begin{array}{l}\text { Mallard duck } \\
\text { (Anas platyrhynchos) }\end{array}$} & $\begin{array}{l}\text { sodium } \\
\text { arsenite }\end{array}$ & NR & 323 & $\begin{array}{l}\mathrm{LD}_{s 0} \\
\text { (single dose) }\end{array}$ & NAS, 1977 \\
\hline & $\begin{array}{l}\text { sodium } \\
\text { arsenite }\end{array}$ & 500 & NR & 32-day $L D_{s 0}$ & NAS, 1977 \\
\hline $\begin{array}{l}\text { California quail } \\
\text { (Callipepla californica) }\end{array}$ & $\begin{array}{l}\text { sodium } \\
\text { arsenite }\end{array}$ & NR & 47.6 & $\mathrm{LD}_{30}$ & Hudson et al., 1984 \\
\hline $\begin{array}{l}\text { Ring-necked pheasant } \\
\text { (Phasianus colchicus) }\end{array}$ & $\begin{array}{l}\text { sodium } \\
\text { arsenite }\end{array}$ & NR & 386 & $\begin{array}{l}\mathrm{LD}_{50} \\
\text { (single dose) }\end{array}$ & Hudson et al., 1984 \\
\hline
\end{tabular}

- Source of data and references: Eisler, 1988.

NR. Not reported.

Table 5. Toxicity of trivalent arsenic compounds to domestic animals

\begin{tabular}{|c|c|c|c|c|c|}
\hline Species & Chemical & $\begin{array}{l}\text { Conc. in Diet } \\
\text { or Water }\end{array}$ & Dose $^{d}$ & Effect & Reference \\
\hline \multirow[t]{2}{*}{ Cattle } & arsenic trioxide & NR & $\begin{array}{l}33-55 \mathrm{mg} / \mathrm{kg} \\
\text { (single dose) }\end{array}$ & toxic & $\begin{array}{l}\text { Robertson } \\
\text { et al., } 1984\end{array}$ \\
\hline & sodium arsenite & NR & $1-4$ g/animal & lethal & NRCC, 1978 \\
\hline \multirow[t]{2}{*}{ Sheep } & sodium arsenite & NR & $\begin{array}{l}5-12 \mathrm{mg} / \mathrm{kg} \\
\text { (single dose) }\end{array}$ & acutely toxic & NRCC, 1978 \\
\hline & "total arsenic" & $\begin{array}{l}58 \mathrm{mg} \mathrm{As} / \mathrm{kg} \text { food } \\
(3 \mathrm{wk})\end{array}$ & NR & $\begin{array}{l}\text { no adverse } \\
\text { effects }\end{array}$ & Woolson, 1975 \\
\hline Horse & sodium arsenite & NR & $\begin{array}{l}2-6 \mathrm{mg} / \mathrm{kg} / \mathrm{day} \\
(14 \mathrm{wk})\end{array}$ & lethal & NRCC, 1978 \\
\hline Pig & sodium arsenite & $500 \mathrm{mg} \mathrm{As} / \mathrm{L}$ & $100-200 \mathrm{mg} / \mathrm{kg}$ & lethal & NAS, 1977 \\
\hline Cat & arsenite & NR & $1.5 \mathrm{mg} / \mathrm{kg} /$ day & $\begin{array}{l}\text { chronic toxic } \\
\text { effects }\end{array}$ & $\begin{array}{l}\text { Pershagen and } \\
\text { Vahter, } 1979\end{array}$ \\
\hline \multirow[t]{3}{*}{ Dog } & sodium arsenite & NR & $\begin{array}{l}50-150 \\
\text { mg/animal }\end{array}$ & lethal & NRCC, 1978 \\
\hline & sodium arsenite & $\begin{array}{l}125 \mathrm{mg} \mathrm{As} / \mathrm{kg} \\
\text { food ( } 2 \text { year) }\end{array}$ & $\begin{array}{l}3.0 \mathrm{mg} \\
\mathrm{As} / \mathrm{kg} / \mathrm{day}^{\mathrm{e}}\end{array}$ & $\begin{array}{l}\text { reduced } \\
\text { survival }\end{array}$ & $\begin{array}{l}\text { Byron et al., } \\
1967\end{array}$ \\
\hline & sodium arsenite & $\begin{array}{l}50 \mathrm{mg} \mathrm{As} / \mathrm{kg} \text { food } \\
\text { (2 year) }\end{array}$ & $\begin{array}{l}1.2 \mathrm{mg} \\
\mathrm{As} / \mathrm{kg} / \mathrm{day}\end{array}$ & NOAEL & $\begin{array}{l}\text { Byron et al., } \\
1967\end{array}$ \\
\hline
\end{tabular}


Table 5. (continued)

\begin{tabular}{|c|c|c|c|c|c|}
\hline Species & Chemical & $\begin{array}{l}\text { Conc, in Diet } \\
\text { or Water }\end{array}$ & Dose ${ }^{d}$ & Effect & Reference \\
\hline & sodium arsenite & NR & $\begin{array}{l}4 \mathrm{mg} / \mathrm{kg} / \text { day } \\
\text { (58 days) } \\
+8 \mathrm{mg} / \mathrm{kg} \\
(125 \text { days })\end{array}$ & $\begin{array}{l}\text { LOAEL; } \\
\text { liver enzyme } \\
\text { changes }\end{array}$ & $\begin{array}{l}\text { Neiger and } \\
\text { Osweiler, } 1989\end{array}$ \\
\hline Mammals & arsenic trioxide & NR & $3-250 \mathrm{mg} / \mathrm{kg}$ & lethal & NAS, 1977 \\
\hline Mammals & sodium arsenite & NR & $1-25 \mathrm{mg} / \mathrm{kg}$ & lethal & NAS, 1977 \\
\hline \multirow{2}{*}{$\begin{array}{l}\text { Chicken } \\
\text { (Gallus } \\
\text { gallus) }\end{array}$} & arsenite & NR & $\begin{array}{l}0.01-1.0 \mu \mathrm{g} \\
\text { As/embryo }\end{array}$ & $\leq 34 \%$ dead & NRCC, 1978 \\
\hline & arsenite & NR & $\begin{array}{l}0.03-0.3 \mu \mathrm{g} \\
\text { As/embryo }\end{array}$ & malform. & NRCC, 1978 \\
\hline
\end{tabular}

- Sources of data and references: USAF, 1990; Eisler, 1988 . NR Not reported.

- Dietary level given as $\mathrm{mg} / \mathrm{kg}$ food.

- Concentration in water given as mg/L.

- Dose, in $\mathrm{mg} / \mathrm{kg}$ bw/day, refers to compound unless otherwise stated.

- Calculated using body weight of $12.7 \mathrm{~kg}$ and Equations 8,9 and 10.

Table 6. Toxicity of trivalent arsenic compounds to laboratory animals

\begin{tabular}{|c|c|c|c|c|c|}
\hline Species & Chemical & $\begin{array}{l}\text { Conc. in Diet } \\
\text { or Water }\end{array}$ & $\begin{array}{c}\text { Dose } \\
\text { (mg As/kg) }\end{array}$ & Effect & Reference \\
\hline \multirow[t]{5}{*}{ Rat } & arsenic trioxide & NR & 15.1 (1 dose $)$ & $\mathrm{LD}_{50}$ & Harrison et al., 1958 \\
\hline & sodium arsenite & $\begin{array}{l}125 \mathrm{mg} \mathrm{As} / \mathrm{kg} \text { food } \\
\text { (2 year) }\end{array}$ & $10^{\circ}$ & $\begin{array}{l}\text { FEL, bile duct } \\
\text { enlargement }\end{array}$ & Byron et al., 1967 \\
\hline & sodium arsenite & $\begin{array}{l}62.5 \mathrm{mg} \mathrm{As} / \mathrm{kg} \text { food } \\
(2 \text { year) }\end{array}$ & $5^{c}$ & $\begin{array}{l}\text { reduced growth in } \\
\text { females; no effect on } \\
\text { survival }\end{array}$ & Byron et al., 1967 \\
\hline & sodium arsenite & $\begin{array}{l}31.25 \mathrm{mg} \mathrm{As} / \mathrm{kg} \text { food } \\
\text { (2 year) }\end{array}$ & $2.5^{\mathrm{c}}$ & NOAEL & Byron et al., 1967 \\
\hline & sodium arsenite & $\begin{array}{l}5 \mathrm{mg} \mathrm{As} / \mathrm{L} \\
\text { (lifetime) }\end{array}$ & $0.65^{d}$ & NOAEL & $\begin{array}{l}\text { Schroeder et al., } \\
1968 \mathrm{a}\end{array}$ \\
\hline \multirow[t]{4}{*}{ Mouse } & arsenic trioxide & NR & 39.4 (1 dose $)$ & $\mathbf{L D}_{50}$ & Harrison et al., 1958 \\
\hline & sodium arsenite & NR & $\begin{array}{l}\text { a. } 23 \text { (1 dose }) \\
\text { b. } 11.5 \text { (1 dose })\end{array}$ & $\begin{array}{l}\text { a. Fetal mortality } \\
\text { b. NOAEL }\end{array}$ & Baxley et al., 1981 \\
\hline & arsenic trioxide & $\begin{array}{l}75.8 \mathrm{mg} \mathrm{As} / \mathrm{L} \\
\text { (lifetime) }\end{array}$ & $18.95^{\mathrm{d}}$ & $\begin{array}{l}\text { LOAEL; mild } \\
\text { hyperkeratosis/epi- } \\
\text { dermal hyperplasia }\end{array}$ & Baroni et al., 1963 \\
\hline & soluble arsenite & $\begin{array}{l}5 \mathrm{mg} \mathrm{As} / \mathrm{L}+ \\
0.06 \mathrm{mg} \mathrm{As} / \mathrm{kg} \text { food } \\
\text { ( } 3 \text { generations) }\end{array}$ & $1.26^{\mathrm{cod}}$ & $\begin{array}{l}\text { LOAEL; incr. in } \\
\text { male to female ratio; } \\
\text { decr. in litter size }\end{array}$ & $\begin{array}{l}\text { Schroeder and } \\
\text { Mitchener, } 1971\end{array}$ \\
\hline
\end{tabular}


18

Table 6. (continued)

\begin{tabular}{|c|c|c|c|c|c|}
\hline Species & Chemical & $\begin{array}{l}\text { Conc. in Diet" } \\
\text { or Water }\end{array}$ & $\begin{array}{c}\text { Dose } \\
\text { (mg As/kg) }\end{array}$ & Effect & Reference \\
\hline & sodium arsenite & $\begin{array}{l}5 \mathrm{mg} \mathrm{As} / \mathrm{L}+ \\
0.46 \mathrm{mg} \mathrm{As} / \mathrm{kg} \text { food } \\
\text { (lifetime) }\end{array}$ & $0.44^{c, d}$ & $\begin{array}{l}\text { LOAEL; slight decr. } \\
\text { in median life span; } \\
\text { no effect on growth }\end{array}$ & $\begin{array}{l}\text { Schroeder and } \\
\text { Balassa, } 1967\end{array}$ \\
\hline & sodium arsenite & $\begin{array}{l}0.5 \mathrm{mg} \mathrm{As} / \mathrm{L} \\
(3 \text { weeks) }\end{array}$ & $0.125^{\mathrm{d}}$ & $\begin{array}{l}\text { LOAEL; } \\
\text { immunosuppressive } \\
\text { effects }\end{array}$ & Blakely et al., 1980 \\
\hline
\end{tabular}

- Dietary level in $\mathrm{mg} / \mathrm{kg}$ food.

- Concentration in water given as $\mathrm{mg} / \mathrm{L}$.

- Estimated using reference body weight (see Table 1) and Equations 8, 9, and 10.

d Estimated using reference body weight (see Table 1) and Equations 19, 20 and 21.

\subsubsection{Extrapolations to Wildlife Species}

Estimates of benchmarks for wildlife are shown in Table 7. The values derived from laboratory studies are shaded. The NOAELs for dose $(\mathrm{mg} / \mathrm{kg}$ bw/day) were estimated using Equation 4. Concentrations in food $\left(\mathrm{C}_{f}\right)$ equivalent to the NOAEL were calculated using the food factors listed in Table 1 and Equation 8. Similarly, concentrations in water $\left(\mathrm{C}_{w}\right)$ equivalent to the NOAELs were estimated from the water factors given in Table 1 and Equation 20.

Three of the toxicity values listed in Tables 5 and 6 were used to estimate benchmarks for wildlife; the drinking water LOAEL of $5 \mathrm{mg} / \mathrm{L}$ for mice (Schroeder and Mitchener, 1971); the dietary NOAEL of $62.5 \mathrm{ppm}$ for rats (Byron et al., 1967); and a dietary NOAEL of $50 \mathrm{ppm}$ for dogs (Bryon et al., 1967). These values were used to estimate NOAELs, $C_{f}$, and $C_{w}$ for the white-footed mouse, cotton rat, red fox, and whitetail deer (Table 7). As expected, benchmarks derived from related species are similar because of similarities in body weight and food and water consumption. Wildlife benchmarks derived from the mouse study are substantially lower than the corresponding NOAELs, $C_{f} s$, and $C_{w} s$ derived from the rat or dog studies. There may be several explanations for these differences. Mice may be unusually sensitive to trivalent arsenic; however, the $\mathrm{LD}_{\mathrm{so}}$ data for rats and mice suggest a similar level of tolerance. The mouse study was a three-generation bioassay in which reproductive effects (reduced litter size) were identified. Although both the rat and dog studies involved chronic exposure durations, neither evaluated potential reproductive effects. Therefore, it is possible that reproductive effects similar to those seen in mice might occur in rats and dogs at or below the experimental NOAELs for these species if multigeneration studies were conducted. Another possibility is that trivalent arsenic may be relatively more toxic in drinking water than food, which might be the case if there were significant differences in rates of gastrointestinal absorption. If this can be shown to be the case, 
19

Table 7. Selected wildlife toxicity values for trivalent inorganic arsenic, a,b

\begin{tabular}{|c|c|c|c|c|c|c|c|c|c|}
\hline \multirow[b]{2}{*}{ Species } & \multirow[b]{2}{*}{$\begin{array}{l}\text { BW } \\
(\mathrm{kg})\end{array}$} & \multirow[b]{2}{*}{$\begin{array}{c}\text { Food } \\
\text { factor } f^{z}\end{array}$} & \multirow[b]{2}{*}{$\begin{array}{c}\text { Water factor } \\
\qquad \omega^{\sigma}\end{array}$} & \multirow[b]{2}{*}{ LOAEL } & \multicolumn{3}{|c|}{ NOAEL (as As) } & \multirow[b]{2}{*}{$\begin{array}{c}\mathbf{L D}_{\text {s0 }} \\
\text { (mg As/kg) }\end{array}$} & \multirow[b]{2}{*}{$\frac{\text { NOAEL }}{\mathbf{L D}_{30}}$} \\
\hline & & & & & $\begin{array}{c}\text { Dose } \\
(\mathrm{mg} / \mathrm{kg})\end{array}$ & $\underset{(\mathrm{mg} / \mathrm{kg})}{\mathrm{C}_{\mathrm{f}}^{\left({ }^{\circ}\right.}}$ & $\begin{array}{c}\mathrm{C}_{w}^{(\infty)} \\
(\mathrm{mg} / \mathrm{L})\end{array}$ & & \\
\hline Mouse & 0.030 & 0.18 & 0.25 & $0 \%$ & $0.126^{(20)}$ & 0.7 & $0.5^{(s)}$ & $\% \%$ & 0.002 \\
\hline White-footed mouse & 0.022 & 0.155 & 0.3 & & & & & & \\
\hline Rat & 0.35 & 0.05 & 0.13 & & $5^{(\S)}$ & 62 & 38.5 & \% & 0.21 \\
\hline \multirow[t]{3}{*}{ Cotton rat } & 0.15 & $0.070^{(14,5)}$ & $0.12^{211}$ & & & & & & \\
\hline & \multicolumn{4}{|c|}{ Extrapolated from data for laboratory rat $\rightarrow$} & $6.6^{(4)}$ & 95 & 55 & & \\
\hline & \multicolumn{4}{|c|}{ Extrapolated from data for laboratory mouse $\rightarrow$} & $0.07^{(4)}$ & 1.0 & 0.6 & & \\
\hline Dog & 12.7 & 0.024 & 0.051 & & $1.2^{\circ}$ & 30 & 26 & & \\
\hline \multirow[t]{3}{*}{ Red fox } & 4.5 & 0.1 & 0.084 & & & & & & \\
\hline & \multicolumn{4}{|c|}{ Extrapolated from data for dog $\rightarrow$} & $1.7^{(4)}$ & 17 & 20 & & \\
\hline & \multicolumn{4}{|c|}{ Extrapolated from data for laboratory mouse $\rightarrow$} & $0.024^{(4)}$ & 0.24 & 0.28 & & \\
\hline \multirow[t]{4}{*}{ Whitetail deer } & 56.5 & 0.031 & 0.065 & & & & & $\% 10 \%$ & \\
\hline & \multicolumn{4}{|c|}{ Extrapolated from data for laboratory rat $\rightarrow$} & $0.9^{(4)}$ & 29 & 13.8 & & \\
\hline & \multicolumn{4}{|c|}{ Extrapolated from data for $\mathrm{dog} \rightarrow$} & $0.73^{(4)}$ & 23.5 & 11.2 & & \\
\hline & \multicolumn{4}{|c|}{ Extrapolated from data for laboratory mice $\rightarrow$} & $0.01^{(4)}$ & 0.32 & 0.15 & & \\
\hline
\end{tabular}

- Numbers in parentheses refer to equations in text used to derive the values.

- Shaded values are experimentally derived. 'soe Table 1. 
then benchmarks based on media-specific studies would be appropriate. Because there is insufficient information to determine which of these factors is responsible, the conservative approach would be to use the mouse data to estimate the benchmarks for the wildlife species.

\subsection{POLYCHLORINATED BIPHENYLS}

Polychlorinated biphenyls occur in a variety of different formulations consisting of mixtures of individual compounds. The most well-known of these formulations is the Aroclor series (i.e., Aroclor 1016, Aroclor 1242, Aroclor 1248, Aroclor 1254, etc.). The Aroclor formulations vary in the percent chlorine, and, generally, the higher the chlorine content the greater the toxicity. This analysis will focus on Aroclor 1254 for which chronic toxicity data are available for two species of wildlife.

\subsubsection{Toxicity to Wildlife}

Toxicity data for Aroclor 1254 are available for two species of wildlife: white-footed mice and mink (Table 8). In both species, the reproductive system and developing embryos are adversely affected by both acute and chronic exposures. A dietary LOAEL of $10 \mathrm{ppm}$ was reported for white-footed mice (Linzey, 1987). Using Equation 5, a body weight of $0.22 \mathrm{~kg}$ (Table 1) and a food consumption rate of $3.4 \mathrm{~g} /$ day (Table 1), the estimated NOAEL for this species would be $\geq 0.155 \mathrm{mg} / \mathrm{kg}$ bw/day. A dietary NOAEL of $1 \mathrm{ppm}$ was reported for mink (Aulerich and Ringer, 1977). Using a time-weighted average body weight of $0.8 \mathrm{~kg}$ (Bleavins et al. 1980) and a food consumption rate of $110 \mathrm{~g} /$ day $(137 \mathrm{~g} / \mathrm{kg}$ bw/day $\times 0.8 \mathrm{~kg}$ bw; Bleavins and Aulerich 1981), the NOAEL is $0.137 \mathrm{mg} / \mathrm{kg} / \mathrm{day}$.

\subsubsection{Toxicity to Domestic Animals}

No information was found in the available literature on the toxicity of Aroclor 1254 to domestic animals.

\subsubsection{Toxicity to Laboratory Animals}

As shown in Table 9, laboratory studies have identified a dietary NOAEL of $5 \mathrm{ppm}(=0.4$ $\mathrm{mg} / \mathrm{kg} \mathrm{bw/day)} \mathrm{for} \mathrm{rats} \mathrm{exposed} \mathrm{to} \mathrm{Aroclor} 1254$ over two generations (Linder et al., 1974). Reported LOAELs are 4-10 times higher than the NOAEL, and the single-dose LD $_{s 0}$ is about 4000-fold higher than the NOAEL. As shown by the dose levels that produce fetotoxicity during gestation, rabbits appear to be less sensitive than rats.

\subsubsection{Extrapolations to Wildlife Species}

Experimentally derived and extrapolated toxicity values for Aroclor 1254 for representative wildlife species are shown in Table 10. Empirical data are available for three species: laboratory rat (Linder et al., 1974), white-footed mouse (Linzey, 1987) and mink (Aulerich and Ringer, 1977). Reproductive and/or developmental changes were the endpoints evaluated in each of these studies. 
21

Table 8. Toxicity of Aroclor 1254 to wildlife

\begin{tabular}{|c|c|c|c|c|c|}
\hline Species & $\begin{array}{c}\text { Concentration } \\
\text { in Food }\end{array}$ & $\begin{array}{l}\text { Daily Dose } \\
\text { (mg/kg) }\end{array}$ & $\begin{array}{l}\text { Expos. } \\
\text { Period }\end{array}$ & Effect & Reference \\
\hline \multirow[t]{3}{*}{$\begin{array}{l}\text { White-footed } \\
\text { mouse }\end{array}$} & 400 ppm & $62^{a}$ & $2-3$ wk & FEL, reprod. & $\begin{array}{l}\text { Sanders and } \\
\text { Kirkpatrick, } 1975\end{array}$ \\
\hline & $200 \mathrm{ppm}$ & $31^{\circ}$ & $60 d$ & $\begin{array}{l}\text { LOAEL, } \\
\text { reproduction }\end{array}$ & $\begin{array}{l}\text { Merson and } \\
\text { Kirkpatrick, } 1976\end{array}$ \\
\hline & $10 \mathrm{ppm}$ & $1.55^{\mathrm{a}}$ & $18 \mathrm{mo}$ & $\begin{array}{l}\text { LOAEL, } \\
\text { reproduction }\end{array}$ & Linzey, 1987 \\
\hline \multirow[t]{3}{*}{$\operatorname{mink}$} & $6.5 \mathrm{ppm}$ & $0.89^{a}$ & $9 \mathrm{mo}$ & $\mathrm{LC}_{50}$ & $\begin{array}{l}\text { Ringer et al., 1981; } \\
\text { ATSDR, } 1989\end{array}$ \\
\hline & $2 \mathrm{ppm}$ & $\begin{array}{l}0.38^{b} \\
0.28^{c}\end{array}$ & $9 \mathrm{mo}$ & $\begin{array}{l}\text { FEL/LOAEL, } \\
\text { fetotoxicity }\end{array}$ & $\begin{array}{l}\text { Aulerich and Ringer, } \\
1977\end{array}$ \\
\hline & $1 \mathrm{ppm}$ & $0.137^{c}$ & $5 \mathrm{mo}$ & NOAEL & $\begin{array}{l}\text { Aulerich and Ringer, } \\
1977\end{array}$ \\
\hline
\end{tabular}

Estimated from Equation 8 using a food factor of 0.155 .

beported by ATSDR (1989); based on food intake of $150 \mathrm{~g} /$ day and mean body weight of $0.8 \mathrm{~kg}$

' Estimated a food consumption rate of $110 \mathrm{~g} / \mathrm{d}$ and a body weight of $0.8 \mathrm{~kg}$ (as reported by Bleavins et al., 1980).

Table 9. Toxicity of Aroclor 1254 to laboratory animals

\begin{tabular}{|c|c|c|c|c|c|}
\hline Species & $\begin{array}{c}\text { Concentration } \\
\text { in Diet }\end{array}$ & $\begin{array}{c}\text { Daily Dose } \\
\text { (mg/kg) }\end{array}$ & $\begin{array}{c}\text { Exposure } \\
\text { Period }\end{array}$ & Effect & Reference \\
\hline \multirow[t]{5}{*}{ Rat } & & 1010 & 1 day & $\mathrm{LD}_{50}$ & Garthoff et al., 1981 \\
\hline & $50 \mathrm{ppm}$ & $4^{a}$ & During gestation & $\begin{array}{l}\text { LOAEL, for } \\
\text { fetotoxicity }\end{array}$ & Collins and Capen, 1980 \\
\hline & 25. ppm & $2^{a}$ & 104 week & $\begin{array}{l}\text { LOAEL, reduced } \\
\text { survival }\end{array}$ & $\begin{array}{l}\text { NCI, 1978; } \\
\text { ATSDR, 1989a }\end{array}$ \\
\hline & $20 \mathrm{ppm}$ & $1.6^{\mathrm{a}}$ & 2 generations & $\begin{array}{l}\text { FEL/LOAEL, } \\
\text { reduced litter size }\end{array}$ & Linder et al., 1974 \\
\hline & 5 ppm & $0.4^{\mathrm{a}}$ & 2 generations & NOAEL & Linder et al., 1974 \\
\hline \multirow[t]{2}{*}{ Rabbit } & & 10.0 & $\begin{array}{l}\text { During gestation } \\
\text { ( } 28 \text { days) }\end{array}$ & $\begin{array}{l}\text { NOAEL for } \\
\text { fetoxicity }\end{array}$ & Villeneuve et al., 1971 \\
\hline & & 12.5 & $\begin{array}{l}\text { During gestation } \\
\text { ( } 28 \text { days) }\end{array}$ & FEL, fetal deaths & Villeneuve et al., 1971 \\
\hline
\end{tabular}

- Calculated using a food factor of 0.08 (see Table 1) and Equation 8. 
Table 10. Selected wildlife toxicity values for Aroclor 1254,b

\begin{tabular}{|c|c|c|c|c|c|c|c|c|c|}
\hline \multirow[b]{2}{*}{ Species } & \multirow[b]{2}{*}{$\begin{array}{r}\text { bw } \\
(\mathbf{k g})\end{array}$} & \multirow[b]{2}{*}{$\begin{array}{c}\text { Food factor } \\
f f\end{array}$} & \multirow[b]{2}{*}{$\begin{array}{c}\text { Water factor } \\
\omega \\
\end{array}$} & \multirow[b]{2}{*}{$\begin{array}{c}\begin{array}{c}\text { LOAEL } \\
\text { (ppm diet) }\end{array} \\
\end{array}$} & \multirow[b]{2}{*}{$\begin{array}{c}\text { NOAEL } \\
(\mathrm{mg} / \mathrm{kg} / \mathrm{d})\end{array}$} & \multicolumn{2}{|c|}{ Benchmarks } & \multirow[b]{2}{*}{$\begin{array}{c}\mathbf{L D}_{s 0} \\
(\mathbf{m g} / \mathbf{k g})\end{array}$} & \multirow[b]{2}{*}{$\begin{array}{c}\text { NOAEL/ } \\
\text { LD }_{30}\end{array}$} \\
\hline & & & & & & $\begin{array}{c}C_{f} \\
\text { (mg/kg food) }\end{array}$ & $\begin{array}{c}\mathrm{C}_{\mathrm{w}} \\
(\mathrm{mg} / \mathrm{L})\end{array}$ & & \\
\hline Rat (lab) & 0.35 & 0.08 & 0.13 & & $0.4^{(n)}$ & $\$ 8$ & 3.1 & \%olo & 0.0004 \\
\hline \multirow[t]{3}{*}{ White-footed mouse } & 0.022 & 0.155 & 0.3 & $\%$ & $\geq 0.155^{(1)}$ & 1.0 & 0.52 & & \\
\hline & \multicolumn{4}{|c|}{ Extrapolated from rat data $\rightarrow$} & $1.01^{(0)}$ & $6.5^{(\mathrm{)})}$ & 3.35 & & \\
\hline & \multicolumn{4}{|c|}{ Extrapolated from mink data $\rightarrow$} & $0.45^{(0)}$ & $2.9^{(n)}$ & 1.50 & & \\
\hline \multirow[t]{3}{*}{ Mink } & $0.80^{c}$ & 0.137 & 0.099 & & $0.137^{\infty}$ & \% & 0.71 & $1 \%$ & 0.06 \\
\hline & \multicolumn{4}{|c|}{ Extrapolated from mouse data $\rightarrow$} & $\geq 0.05^{(1)}$ & $0.34^{m}$ & $0.47^{(20)}$ & & \\
\hline & \multicolumn{4}{|c|}{ Extrapolated from rat data $\rightarrow$} & $0.30^{(4)}$ & $2.22^{m}$ & $3.08^{201}$ & & \\
\hline \multirow[t]{4}{*}{ Cotton rat: } & 0.15 & 0.07 & 0.12 & & & & & & \\
\hline & \multicolumn{4}{|c|}{ Extrapolated from mouse data $\rightarrow$} & $\geq 0.08^{(4)}$ & $1.17^{\mathrm{m}}$ & $0.68^{(20)}$ & & \\
\hline & \multicolumn{4}{|c|}{ Extrapolated from rat data $\rightarrow$} & $0.53^{(4)}$ & $7.56^{\circ}$ & $4.41^{(20)}$ & & \\
\hline & \multicolumn{4}{|c|}{ Extrapolated from mink data $\rightarrow$} & $0.24^{(4)}$ & $3.4^{(x)}$ & $1.98^{200}$ & & \\
\hline \multirow[t]{4}{*}{ Whitetail deer: } & 56.5 & 0.031 & 0.065 & & & & & & \\
\hline & \multicolumn{4}{|c|}{ Extrapolated from mouse data $\rightarrow$} & $\geq 0.012^{(4)}$ & $0.37^{n}$ & $0.17^{(00)}$ & & \\
\hline & \multicolumn{4}{|c|}{ Extrapolated from rat data $\rightarrow$} & $0.075^{(4)}$ & $2.43^{(*)}$ & $1.14^{(00)}$ & & \\
\hline & \multicolumn{4}{|c|}{ Extrapolated from mink data $\rightarrow$} & $0.034^{(4)}$ & $1.09^{(n)}$ & $0.51^{(20)}$ & & \\
\hline
\end{tabular}

- Numbers in parentheses refer to equations in text.

- Shaded values are experimentally derived.

- TWA bw for females to 10 mo (reproductive maturity) (EPA, 1988a). 
The calculated NOAELs are $0.4 \mathrm{mg} / \mathrm{kg}$ bw/day for the rat, $0.155 \mathrm{mg} / \mathrm{kg}$ bw/day for the white-footed mouse, and $0.137 \mathrm{mg} / \mathrm{kg} \mathrm{bw} / \mathrm{day}$ for mink. These data indicate that the laboratory rat is less sensitive to the toxicity of Aroclor 1254 than either the white-footed mouse or the mink.

The most conservative benchmark for Aroclor 1254 would be the NOAEL for whitetail deer $(0.012 \mathrm{mg} / \mathrm{kg} \mathrm{bw} /$ day) extrapolated from the data for the white-footed mouse. The NOAEL derived from the mink data $(0.034 \mathrm{mg} / \mathrm{kg})$ may be more reliable because it was based on an experimentally derived NOAEL whereas the white-footed mouse value was based on an experimentally derived LOAEL. However, because metabolism and physiology are more likely to be similar between an omnivore (mouse) and a herbivore (deer) than between a carnivore (mink) and herbivore, the white-footed mouse NOAEL may be a better estimate of toxicity to whitetail deer than the mink NOAEL.

For mink, a combined water quality benchmark for Aroclor 1254 can be derived from Equation 26. Using a $\log P_{\text {oct }}$ of 6.5 (ATSDR, 1989), the bioconcentration factor (BCF) for Aroclor 1254 was estimated from Equation 27 to be 51,286. Conservatively, the diet of mink is assumed to consist entirely of small fish (trophic level 3, FCM $=45.0$; Table 2); therefore, the BAF was estimated to be 2,307,876. For mink weighing $0.8 \mathrm{~kg}$ and a NOAEL of $0.137 \mathrm{mg} / \mathrm{kg}$, the combined food and water benchmark for Aroclor 1254 is calculated to be $0.43 \mathrm{ng} / \mathrm{L}$.

\section{SITE-SPECIFIC CONSIDERATIONS}

The examples given in this report for trivalent inorganic arsenic and Aroclor 1254 illustrate the extent of the analysis that is required for an understanding of the toxicity of environmental contaminants to wildlife and for the development of benchmark values. For a complete risk assessment at a particular site, similar analyses would be needed for all the chemicals present, as well as information on their physical and chemical state, their concentration in various environmental media, and their bioavailability. The last factor is especially important in estimating environmental impacts. For example, insoluble substances tightly bound to soil particles are unlikely to be taken up by organisms even if ingested. In addition, the chemical or valence state of a contaminant may alter its toxicity such that the different chemical or valence states may have to be treated separately as in the case of trivalent arsenic. Similar problems can be encountered with formulations consisting of mixtures of compounds such as the Aroclors, and each may have to be evaluated separately, unless the relative potency of each of the components can be determined.

For a site-specific assessment, information on the types of wildlife species present, their average body size, and food and water consumption rates would also be needed for calculating NOAELs and environmental criteria. Use of observed values for food and water consumption (if available) are recommended over rates estimated by allometric equations. A list of pertinent exposure parameters (body weights, food and water consumption rates) for selected avian and mammalian species for the DOE Oak Ridge site is given in Appendix B. Exposure information for additional wildlife species may be found in Wildlife Exposure Factors Handbook (EPA, 1993a and 1993b). Since body size of some species can vary geographically, the more specific the data 
are to the local population, the more reliable will be the estimates. Data on body size are especially important in the extrapolation procedure, particularly if calculations of the NOAEL and environmental concentrations are based solely on the adjustment factor as shown in Equation 4. In such cases the lowest NOAEL will be derived from the species with the largest body size. Estimates of average body weights for wildlife species used herein were obtained from the available literature (Appendix B, see also Table 1). These were used to calculate body surface area scaling factors from Equation 4 (Table 11) and also to derive food factors from Equation 10 and water factors from Equations 21 (see Table 1).

Information on physiological, behavioral, or ecological characteristics of these species can also be of special importance in determining if certain species are particularly sensitive to a particular chemical or groups of chemicals. If one species occurring at a site is known to be unusually sensitive to a particular contaminant, then the criteria should be based on data for that species (with exceptions noted in the following paragraphs). Similarly, extrapolations from studies on laboratory animals should be based on the most sensitive species unless there is evidence that this species is unusually sensitive to the chemical.

Physiological and biochemical data may be important in determining the mechanism whereby a species' sensitivity to a chemical may be enhanced or diminished. Such information would aid in determining whether data for that species would be appropriate for developing criteria for other species.

For example, if the toxic effects of a chemical are related to the induction of a specific enzyme system, as is the case with PCBs, then it would be valuable to know whether physiological factors (enzyme activity levels per unit mass of tissue or rates of synthesis of the hormones affected by the induced enzymes) in the most sensitive species are significantly different from those of other species of wildlife. Furthermore, if the most sensitive species, or closely related species, do not occur at a particular site, then a less stringent criterion might be acceptable.

Physiological data may also reveal how rates of absorption and bioavailability vary with exposure routes and/or exposure conditions. Gastrointestinal absorption may be substantially different depending on whether the chemical is ingested in the diet or in drinking water. Therefore, a NOAEL based on a laboratory drinking water study may be inappropriate to use in extrapolating to natural populations that would only be exposed to the same chemical in their diet. The diet itself may affect gastrointestinal absorption rates. In the case of the mink exposed to PCBs, a diet consisting primarily of contaminated fish in which the PCBs are likely to be concentrated in fatty tissues may result in a different rate of gastrointestinal absorption than that occurring in laboratory rodents dosed with PCBs in dry chow.

Behavioral and ecological data might also explain differences in sensitivity between species. Certain species of wildlife may be more sensitive because of higher levels of environmental stress to which they are subjected. This may be especially true of populations occurring at the periphery of their normal geographic range. Conversely, laboratory animals maintained under stable environmental conditions of low stress may have higher levels of resistance to toxic chemicals. 
As a first step in developing wildlife criteria for chemicals of concern at DOE sites, relevant toxicity data for wildlife and laboratory animals have been compiled (Appendixes $\mathrm{A}$ and $\mathrm{C}$ ). These data consist primarily of NOAELs, LOAELs, and $\mathrm{LD}_{50}$ s for avian and mammalian species. No methodology is currently available for extrapolating from avian or mammalian studies to reptiles and amphibians, and no attempt has been made to do so in this report. No pertinent data on nonpesticide chemicals were found for amphibians, reptiles, or terrestrial invertebrates. Additional chronic exposure studies are needed before toxicological benchmarks can be developed for these groups.

\section{RESULTS}

The results of the analyses are presented in Table 12. Because of the consistency of the body weight differences for the selected mammalian wildlife species, the calculated NOAELs exhibit about a 15-fold range between the species of smallest body size (little brown bat) and that of the largest body size (whitetail deer). In terms of dietary intake, the range in values is much less (2-3 fold) thereby indicating that equivalent dietary levels of a chemical result in nearly equivalent doses between species because food intake is a function of metabolic rate which, in turn, is a function of body size (EPA, 1980a). However, according to EPA, the correlation is not exact because food intake also varies with moisture and caloric content of the food, and it should be noted that in laboratory feeding experiments, the test animals are usually dosed with the chemical in a dry chow. Therefore, it would be expected that the food factor for a species of wildlife would be relatively higher than that of a related laboratory species of comparable body size, resulting in a lower dietary benchmark for wildlife species as compared to that for the related laboratory species.

\section{APPLICATION OF THE BENCHMARKS}

As stated in Sect. 1, ecological risk assessment is a tiered process. As part of the first tier or screening assessment, toxicological benchmarks are used to identify Contaminants of Potential Concern (COPCs) and to focus future data collection. In the second tier or baseline assessment, toxicological benchmarks are one of several lines of evidence used to determine if environmental contaminant concentrations are resulting in ecological effects. In a screening assessment, general, conservative assumptions are made so that all chemicals that may be present at potentially hazardous levels in the environment are retained for future consideration. In contrast, in a baseline assessment, more specific assumptions are made so that an accurate estimate of the contaminant exposure that an individual may experience and potential effects that may result from that exposure may be made.

\subsection{SCREENING ASSESSMENT}

Screening assessments serve to identify those contaminants whose concentrations are sufficiently high such that they may be hazardous to wildlife. The primary emphasis of a screening assessment is to include all potential hazards while eliminating clearly insignificant hazards. To prevent any potential hazards from being overlooked, assumptions made in a screening assessment are conservative. 
Questions that drive a screening assessment include: 1) Which media (water, soil, etc.) are contaminated such that they may be toxic?, 2) What chemicals are involved? (Which contaminants are COPCs)?, 3) What are the concentrations and spatial and temporal distributions of these contaminants?, and 4) What organisms are expected to be significantly exposed to the chemicals? To answer these questions, diet, water, and combined food and water (for aquatic feeding species) benchmark values are compared to the contaminant concentrations observed in the media from the site. If the concentration of a contaminant exceeds the benchmark, it should be retained as a COPC. By comparing contaminant concentrations from several locations within a site to benchmarks for several endpoint species, the spatial extent of potentially hazardous contamination, which media are contaminated, and the species potentially at risk from contamination may be identified.

In a screening assessment, it is generally assumed that wildlife species reside and therefore forage and drink exclusively from the contaminated site. That is, approximately $100 \%$ of the food and water they consume is contaminated. While this assumption simplifies the assessment, due to the mobility and the diverse diets of most wildlife, it is likely to overestimate the actual exposure experienced. It should be remembered, however, that the purpose of the screening assessment is to identify potential risks and data gaps to be filled. Once these data gaps are filled, a definitive evaluation of risk may be made as part of the baseline assessment.

In most screening assessments, because they rely on existing data, available data are likely to be restricted to contaminant concentration in abiotic media (e.g., soil and water). Contaminant concentrations in wildlife foods may need to be estimated using contaminant uptake models such as those described in Baes et al. (1984), Travis and Arms (1988), or Menzies et al. (1992).

Table 13 provides a simplified example of the use of benchmarks in a screening assessment. The purpose of the assessment in this example is to identify the contaminants and media with concentrations sufficiently high to present a hazard to a representative endpoint species (meadow vole). This information will be used to identify gaps in data needed for the baseline assessment. Data consists of the concentrations of four metals in soil and water. These data were compared to values observed at a representative background location and found to be higher. (Screening contaminant concentrations against background helps provide a regional context for the data and aids in identifying anthropogenic contamination. This is particularly important in areas where metal concentrations in native soils are naturally high.) Because dietary exposure cannot be evaluated directly from soil concentrations, metal concentrations in the voles' food (plant foliage) was estimated using plant uptake factors for foliage from Baes et al. (1984). To determine which contaminants pose a risk, a hazard quotient (HQ) was calculated, where HQ $=$ media concentration/benchmark. If $\mathrm{HQ}$ is greater or equal to 1 , contaminant concentrations are sufficiently high that they may produce adverse effects. Contaminants with HQs greater or equal to 1 should be retained as COPCs. In this example, while metal concentrations in water did not exceed any water benchmarks, estimated concentrations of $\mathrm{As}$ and $\mathrm{Hg}$ in plant foliage exceeded dietary benchmarks. These metals should therefore be retained as COPCs in food but not in water. Because contaminant concentrations in plant foliage were estimated, one data need for the baseline assessment consists of actual, measured concentrations in plants. In addition, the form of the metals (i.e., inorganic vs methyl mercury) should be identified so the most appropriate benchmark may be used in the baseline assessment. 


\subsection{BASELINE ASSESSMENT}

In contrast to the screening assessment that defines the scope of the assessment, the baseline assessment uses new and existing data to evaluate the risk of leaving the site unremediated. The purposes of the baseline assessment are to determine 1) if significant ecological effects are occurring at the site, 2) the causes of these effects, 3) the source of the causal agents, and 4) the consequences of leaving the system unremediated. The baseline assessment provides the ecological basis for determining the need for remediation.

Because the baseline assessment focuses on a smaller number of contaminants and species than the screening assessment, it can provide a higher level of characterization of toxicity to the species and communities at the site. In the baseline ERA, a weight-of-evidence approach (Suter, 1993) is employed to determine if and to what degree ecological effects are occurring or may occur. The lines of evidence used in a baseline assessment consist of 1) toxicity tests using ambient media from the site, 2) biological survey data from the site, and 3) comparison of contaminant exposure experienced by endpoint species at the site to wildlife NOAELs.

Estimating the contaminant exposure experienced by wildlife at a waste site consists of summing the exposure received from each separate source. While wildlife may be exposed to contaminants through oral ingestion, inhalation, and dermal absorption, the benchmarks in this document are only applicable to the most common exposure route-oral ingestion. Exposure through inhalation and dermal absorption are special cases that must be considered independently.

The primary routes of oral exposure for terrestrial wildlife are through ingestion of food (either plant or animal) and surface water. In addition, some species may ingest soil incidentally while foraging or purposefully to meet nutrient needs. The total exposure experienced by terrestrial wildlife is represented by the sum of the exposures from each individual source. Total exposure may be represented by the following generalized equation:

where:

$$
E_{\text {total }}=E_{\text {food }}+E_{\text {water }}+E_{\text {soil }}
$$

$\mathrm{E}_{\text {total }}=$ exposure from all sources

$\mathrm{E}_{\text {food }}=$ exposure from food consumption

$\mathrm{E}_{\text {water }}=$ exposure from water consumption

$\mathrm{E}_{\text {soil }} \quad=$ exposure through consumption of soil (either incidental or deliberate)

Building on the screening assessment example, Table 14 provides an example of the use of benchmarks in a baseline assessment. The purpose of the assessment in this example is to ascertain the level of exposure and risk experienced by a representative endpoint species (meadow vole). In addition to soil and water contaminant data, concentrations of $\mathrm{As}, \mathrm{Pb}, \mathrm{Hg}$, and $\mathrm{Se}$ were 
measured in plants on which meadow voles forage. Exposure parameters for each medium were calculated according to the following equation:

$$
E_{\text {media }}=\frac{\text { MCR }(\mathrm{kg} \text { or } \mathrm{L} / \mathrm{d}) \times \mathrm{ACM}(\mathrm{mg} / \mathrm{kg} \text { or } \mathrm{mg} / \mathrm{L})}{\text { Body Weight }(\mathrm{kg})}
$$

where $\mathrm{E}_{\text {medi }}$ = estimated exposure (mg analyte/kg body weight/d) for each medium (e.g., food, water, and soil); $\mathrm{MCR}=$ medium consumption rate; and $\mathrm{ACM}=$ analyte concentration in media. Body weight $(0.044 \mathrm{~kg})$, food $(0.005 \mathrm{~kg} / \mathrm{d})$ and water $(0.006 \mathrm{~L} / \mathrm{d})$ consumption rates for meadow voles were obtained from Appendix B. Beyer et al. (1992) states that soil consumption by meadow voles is $2 \%$ of food consumption. Therefore, soil consumption was estimated to be $2 \%$ of $0.005 \mathrm{~kg} / \mathrm{d}$ or $0.0001 \mathrm{~kg} / \mathrm{d}$. As in the screening assessment, an HQ was calculated in which total exposure was compared to the NOAEL for each contaminant. Total exposure from all sources exceeded NOAELs for both As and Se.

By comparing the exposure from each source (e.g., water, soil, diet) to the NOAEL, the relative contribution of each to the total can be determined. For example, virtually all $\mathrm{Se}$ exposure (98.6\%) was obtained through food consumption; Se exposures from soil and water were both less then the NOAEL. In contrast, As exposure from soil and food both exceeded the NOAEL and accounted for $59 \%$ and $40 \%$ of As exposure, respectively. This information serves not only to identify contaminants that present a risk but by identifying the media that account for the majority of exposure, these data may be used to guide remediation.

In the preceding example, the species used has a small home range $(<1$ ha) and a diet restricted to grassy and herbaceous plant material (Reich, 1981). Therefore, it was assumed that voles would reside and forage exclusively on the hypothetical waste site and that $100 \%$ of the food, water, and soil consumed would be contaminated. Because most wildlife are mobile and many species have varied diets, it is not likely that all food, water, or soil ingested by individuals of other wildlife endpoint species would be obtained from contaminated sources. In the case of species with large home ranges, because they may spend only a portion of their time on a contaminated site (and may receive exposure from multiple, spatially separate locations), their exposure should be represented by the proportion of food, water, or soil obtained from contaminated sources. For species with diverse diets, the contaminant concentrations in the different food types consumed is likely to differ. Dietary exposure for these species would be represented by the sum of the contaminant concentrations in each food type multiplied by the proportion of each food type in the species diet. Ideally, site-specific information on home ranges, diet composition, and use of waste sites by endpoint species should be collected. In the absence of site specific data, information to estimate exposure for selected wildlife species may be found in the Wildlife Exposure Factors Handbook (EPA, 1993a and 1993b)or in other published literature. 
Table 11. Body size scaling factors

\begin{tabular}{|c|c|c|c|c|}
\hline \multicolumn{2}{|c|}{ Experimental Animals } & \multicolumn{2}{|c|}{ Wildife } & \multirow[b]{2}{*}{$\begin{array}{l}\text { Scaling factor } \\
\left(\mathbf{b} w_{t} /\left(b w_{\nabla}\right)^{1 / 3}\right.\end{array}$} \\
\hline Species & $\begin{array}{c}\text { Body Weight" } \\
\left(\mathrm{bw}_{\mathrm{t}} \text {, in kg) }\right.\end{array}$ & Species & $\begin{array}{c}\text { Body weight } \\
\left(\mathrm{bw}_{w} \text { in } \mathbf{k g}\right)\end{array}$ & \\
\hline rat & 0.35 & short-tailed shrew & 0.015 & 2.86 \\
\hline rat & 0.35 & white-footed mouse & 0.022 & 2.52 \\
\hline rat & 0.35 & meadow vole & 0.044 & 2.00 \\
\hline rat & 0.35 & cottontail rabbit & 1.2 & 0.66 \\
\hline rat & 0.35 & mink & 1.0 & 0.70 \\
\hline rat & 0.35 & red fox & 4.5 & 0.43 \\
\hline rat & 0.35 & whitetail deer & 56.5 & 0.18 \\
\hline mouse & 0.03 & short-tailed shrew & 0.015 & 1.26 \\
\hline mouse & 0.03 & white-footed mouse & 0.022 & 1.11 \\
\hline mouse & 0.03 & meadow vole & 0.004 & 0.88 \\
\hline mouse & 0.03 & cottontail rabbit & 1.2 & 0.29 \\
\hline mouse & 0.03 & mink & 1.0 & 0.31 \\
\hline mouse & 0.03 & red fox & 4.5 & 0.19 \\
\hline mouse & 0.03 & whitetail deer & 56.5 & 0.08 \\
\hline
\end{tabular}

- Standard reference values used by EPA.

brom Appendix B. 
Table 12. Toxicological benchmarks for selected avian and mammalian wildlife species

\begin{tabular}{|c|c|c|c|c|c|c|c|}
\hline \multirow{2}{*}{$\begin{array}{l}\text { Contaminant } \\
\text { and Form }\end{array}$} & \multirow{2}{*}{ Test Species } & \multirow{2}{*}{$\begin{array}{c}\text { Test } \\
\text { Species } \\
\text { NOAEL } \\
(\mathbf{m g} / \mathbf{k g} \cdot \mathbf{d})\end{array}$} & \multirow{2}{*}{$\begin{array}{l}\text { Endpoint } \\
\text { Species }^{b}\end{array}$} & \multirow{2}{*}{$\begin{array}{c}\text { Estimated } \\
\text { Wildlife } \\
\text { NOAELc } \\
\left(\mathrm{mg} / \mathbf{k g}^{\circ} \mathrm{d}\right)\end{array}$} & \multicolumn{3}{|c|}{ Toxicological Benchmarks } \\
\hline & & & & & $\begin{array}{c}\text { Diet }^{d} \\
\text { (mg/kg) }\end{array}$ & $\begin{array}{l}\text { Water } \\
\text { (mg/L) }\end{array}$ & $\begin{array}{c}\text { Aquatic } \\
\text { Feeding } \\
\text { Species } \\
(\mathrm{mg} / \mathrm{L})\end{array}$ \\
\hline \multirow[t]{8}{*}{ Acetone } & Rat & 10 & $\begin{array}{l}\text { Short-tailed } \\
\text { Shrew }\end{array}$ & 28.277 & 47.128 & 128.531 & \\
\hline & & & Little Brown Bat & 35.545 & 106.634 & 222.153 & \\
\hline & & & $\begin{array}{c}\text { White-footed } \\
\text { Mouse }\end{array}$ & 24.920 & 161.245 & 83.066 & \\
\hline & & & Meadow Vole & 19.825 & 174.456 & 145.380 & \\
\hline & & & Cottontail Rabbit & 6.659 & 33.717 & 68.887 & \\
\hline & & & Mink & 7.072 & 51.620 & 71.434 & $4.64 e+01$ \\
\hline & & & Red Fox & 4.305 & 43.051 & 50.981 & \\
\hline & & & Whitetail Deer & 1.868 & 60.656 & 28.525 & \\
\hline \multirow[t]{8}{*}{ Aldrin } & Rat & 0.2 & $\begin{array}{l}\text { Short-tailed } \\
\text { Shrew }\end{array}$ & 0.566 & 0.943 & 2.571 & \\
\hline & & & Little Brown Bat & 0.711 & 2.133 & 4.443 & \\
\hline & & & $\begin{array}{l}\text { White-footed } \\
\text { Mouse }\end{array}$ & 0.498 & 3.225 & 1.661 & \\
\hline & & & Meadow Vole & 0.396 & 3.489 & 2.908 & \\
\hline & & & Cottontail Rabbit & 0.133 & 0.674 & 1.378 & \\
\hline & & & Mink & 0.141 & 1.032 & 1.429 & \\
\hline & & & Red Fox & 0.086 & 0.861 & 1.020 & \\
\hline & & & Whitetail Deer & 0.037 & 1.213 & 0.571 & \\
\hline
\end{tabular}


Table 12. (continued)

\begin{tabular}{|c|c|c|c|c|c|c|c|}
\hline \multirow[b]{2}{*}{$\begin{array}{l}\text { Contaminant } \\
\text { and Form }\end{array}$} & \multirow{2}{*}{ Test Species } & \multirow{2}{*}{$\begin{array}{c}\text { Test } \\
\text { Species } \\
\text { NOAEL" } \\
\left(\mathbf{m g} / \mathbf{k g}^{\circ} \mathbf{d}\right)\end{array}$} & \multirow[b]{2}{*}{$\begin{array}{l}\text { Endpoint } \\
\text { Species }\end{array}$} & \multirow{2}{*}{$\begin{array}{c}\text { Estimated } \\
\text { Wildlife } \\
\text { NOAEL }^{c} \\
(\mathrm{mg} / \mathrm{kg} \cdot \mathrm{d})\end{array}$} & \multicolumn{3}{|c|}{ Toxicological Benchmarks } \\
\hline & & & & & $\begin{array}{c}\text { Diet }^{d} \\
(\mathbf{m g} / \mathbf{k g})\end{array}$ & $\begin{array}{c}\text { Watere } \\
\text { (mg/L) }\end{array}$ & $\begin{array}{l}\text { Aquatic } \\
\text { Feeding } \\
\text { Species' } \\
\text { (mg/L) }\end{array}$ \\
\hline Aluminum & Mouse & 1.93 & $\begin{array}{l}\text { Short-tailed } \\
\text { Shrew }\end{array}$ & 2.426 & 4.043 & 11.027 & \\
\hline \multirow[t]{7}{*}{$\mathrm{AlCl}_{3}$} & & & Little Brown Bat & 3.050 & 9.149 & 19.060 & \\
\hline & & & $\begin{array}{c}\text { White-footed } \\
\text { Mouse }\end{array}$ & 2.138 & 13.834 & 7.127 & \\
\hline & & & Meadow Vole & 1.701 & 14.967 & 12.473 & \\
\hline & & & Cottontail Rabbit & 0.571 & 2.893 & 5.910 & \\
\hline & & & Mink & 0.607 & 4.429 & 6.129 & $1.991 \mathrm{e}-02$ \\
\hline & & & Red Fox & 0.369 & 3.694 & 4.374 & \\
\hline & & & Whitetail Deer & 0.160 & 5.204 & 2.447 & \\
\hline Aluminum & Ringed Dove & 111.4 & American Robin & 140.331 & 116.188 & 1019.383 & \\
\hline \multirow[t]{8}{*}{$\mathrm{A} 12\left(\mathrm{SO}_{4}\right) 2$} & & & $\begin{array}{l}\text { American } \\
\text { Woodcock }\end{array}$ & 102.753 & 135.634 & 1017.256 & \\
\hline & & & Wild Turkey & 33.711 & 1123.692 & 1029.065 & \\
\hline & & & $\begin{array}{c}\text { Belted } \\
\text { Kingfisher }\end{array}$ & 113.112 & 223.208 & 1046.288 & $9.65 \mathrm{e}-01$ \\
\hline & & & $\begin{array}{c}\text { Great Blue } \\
\text { Heron }\end{array}$ & 45.167 & 257.022 & 1020.316 & $1.11 \mathrm{e}+00$ \\
\hline & & & Barred Owl & 67.201 & 1029.553 & 1025.172 & \\
\hline & & & Barn Ow1 & 77.469 & 577.606 & 1031.440 & \\
\hline & & & Cooper's Hawk & 79.009 & 1020.150 & 1020.150 & \\
\hline & & & Red-tailed Hawk & 57.901 & 71.645 & 1018.700 & \\
\hline
\end{tabular}


32

Table 12. (continued)

\begin{tabular}{|c|c|c|c|c|c|c|c|}
\hline \multirow[b]{2}{*}{$\begin{array}{l}\text { Contaminant } \\
\text { and Form }\end{array}$} & \multirow{2}{*}{ Test Species } & \multirow{2}{*}{$\begin{array}{c}\text { Test } \\
\text { Species } \\
\text { NOAEL" } \\
\left(\mathbf{m g} / \mathbf{k g}^{\circ} \mathbf{d}\right)\end{array}$} & \multirow[b]{2}{*}{$\begin{array}{l}\text { Endpoint } \\
\text { Species }^{\mathrm{b}}\end{array}$} & \multirow{2}{*}{$\begin{array}{c}\text { Estimated } \\
\text { Wildlife } \\
\text { NOAEL } \\
\left(\mathrm{mg} / \mathrm{kg}^{-} \mathrm{d}\right)\end{array}$} & \multicolumn{3}{|c|}{ Toxicological Benchmarks } \\
\hline & & & & & $\underset{(\mathbf{m g} / \mathbf{k g})}{\mathrm{Diet}^{d}}$ & $\begin{array}{l}\text { Water } \\
\text { (mg/L) }\end{array}$ & $\begin{array}{l}\text { Aquatic } \\
\text { Feeding } \\
\text { Species } \\
\text { (mg/L) }\end{array}$ \\
\hline Antimony & Mouse & 0.125 & $\begin{array}{l}\text { Short-tailed } \\
\text { Shrew }\end{array}$ & 0.157 & 0.262 & 0.714 & \\
\hline \multirow{7}{*}{$\begin{array}{l}\text { Antimony Potassium } \\
\text { Tartrate } \\
\end{array}$} & & & Little Brown Bat & 0.198 & 0.593 & 1.234 & \\
\hline & & & $\begin{array}{l}\text { White-footed } \\
\text { Mouse }\end{array}$ & 0.138 & 0.896 & 0.462 & \\
\hline & & & Meadow Vole & 0.110 & 0.969 & 0.808 & \\
\hline & & & Cottontail Rabbit & 0.037 & 0.187 & 0.383 & \\
\hline & & & Mink & 0.039 & 0.287 & 0.397 & $1.67 e-01$ \\
\hline & & & Red Fox & 0.024 & 0.239 & 0.283 & \\
\hline & & & Whitetail Deer & 0.010 & 0.337 & 0.159 & \\
\hline \multirow[t]{8}{*}{ Aroclor 1016} & Mink & 1.37 & $\begin{array}{l}\text { Short-tailed } \\
\text { Shrew }\end{array}$ & 5.478 & 9.130 & 24.899 & \\
\hline & & & Litule Brown Bat & 6.886 & 20.657 & 43.036 & \\
\hline & & & $\begin{array}{l}\text { White-footed } \\
\text { Mouse }\end{array}$ & 4.827 & 31.237 & 16.092 & \\
\hline & & & Meadow Vole & 3.840 & 33.796 & 28.163 & \\
\hline & & & Cottontail Rabbit & 1.290 & 6.532 & 13.345 & \\
\hline & & & Mink & 1.370 & 10.000 & 13.838 & $1.26 \mathrm{e}-04$ \\
\hline & & & Red Fox & 0.834 & 8.340 & 9.876 & \\
\hline & & & Whitetail Deer & 0.362 & 11.750 & 5.526 & \\
\hline
\end{tabular}


33

Table 12. (continued)

\begin{tabular}{|c|c|c|c|c|c|c|c|}
\hline \multirow{2}{*}{$\begin{array}{l}\text { Contaminant } \\
\text { and Form }\end{array}$} & \multirow{2}{*}{ Test Species } & \multirow{2}{*}{$\begin{array}{c}\text { Test } \\
\text { Species } \\
\text { NOAEL" } \\
\left(\mathbf{m g} / \mathbf{k g}^{\circ} \mathbf{d}\right)\end{array}$} & \multirow{2}{*}{$\begin{array}{l}\text { Endpoint } \\
\text { Species }^{b}\end{array}$} & \multirow{2}{*}{$\begin{array}{c}\text { Estimated } \\
\text { Wildlife } \\
\text { NOAEL } \\
\left(\mathrm{mg} / \mathrm{kg}^{\bullet} \mathrm{d}\right)\end{array}$} & \multicolumn{3}{|c|}{ Toxicological Benchmarks } \\
\hline & & & & & $\begin{array}{c}\text { Diet }^{d} \\
(\mathrm{mg} / \mathrm{kg})\end{array}$ & $\begin{array}{c}\text { Watere } \\
\text { (mg/L) }\end{array}$ & $\begin{array}{l}\text { Aquatic } \\
\text { Feeding } \\
\text { Species } \\
\text { (mg/L) }\end{array}$ \\
\hline \multirow[t]{8}{*}{ Aroclor 1242} & Mink & 0.0685 & $\begin{array}{c}\text { Short-tailed } \\
\text { Shrew }\end{array}$ & 0.274 & 0.456 & 1.245 & \\
\hline & & & Little Brown Bat & 0.344 & 1.033 & 2.152 & \\
\hline & & & $\begin{array}{l}\text { White-footed } \\
\text { Mouse }\end{array}$ & 0.241 & 1.562 & 0.805 & \\
\hline & & & Meadow Vole & 0.192 & 1.690 & 1.408 & \\
\hline & & & Cottontail Rabbit & 0.065 & 0.327 & 0.667 & \\
\hline & & & Mink & 0.069 & 0.500 & 0.692 & $6.28 \mathrm{e}-06$ \\
\hline & & & Red Fox & 0.042 & 0.417 & 0.494 & \\
\hline & & & Whitetail Deer & 0.018 & 0.587 & 0.276 & \\
\hline \multirow[t]{9}{*}{ Aroclor 1242} & Screech OwI & 0.41 & American Robin & 0.544 & 0.450 & 3.949 & \\
\hline & & & $\begin{array}{l}\text { American } \\
\text { Woodcock }\end{array}$ & 0.398 & 0.525 & 3.941 & \\
\hline & & & Wild Turkey & 0.131 & 4.353 & 3.986 & \\
\hline & & & $\begin{array}{c}\text { Belted } \\
\text { Kingfisher }\end{array}$ & 0.438 & 0.865 & 4.053 & $1.09 \mathrm{e}-05$ \\
\hline & & & $\begin{array}{c}\text { Great Blue } \\
\text { Heron }\end{array}$ & 0.175 & 0.996 & 3.952 & $1.25 \mathrm{e}-0.05$ \\
\hline & & & Barred Owl & 0.260 & 3.988 & 3.971 & \\
\hline & & & Barn Owl & 0.300 & 2.237 & 3.995 & \\
\hline & & & Cooper's Hawk & 0.306 & 3.952 & 3.952 & \\
\hline & & & Red-tailed Hawk & 0.224 & 0.278 & 3.946 & \\
\hline
\end{tabular}


34

Table 12. (continued)

\begin{tabular}{|c|c|c|c|c|c|c|c|}
\hline \multirow{2}{*}{$\begin{array}{l}\text { Contaminant } \\
\text { and Form }\end{array}$} & \multirow{2}{*}{ Test Species } & \multirow{2}{*}{$\begin{array}{c}\text { Test } \\
\text { Species } \\
\text { NOAEL } \\
(\mathbf{m g} / \mathbf{k g} \bullet \mathrm{d})\end{array}$} & \multirow{2}{*}{$\begin{array}{l}\text { Endpoint } \\
\text { Species }^{b}\end{array}$} & \multirow{2}{*}{$\begin{array}{l}\text { Estimated } \\
\text { Wildlife } \\
\text { NOAELc } \\
\text { (mg/kg • d) }\end{array}$} & \multicolumn{3}{|c|}{ Toxicological Benchmarks } \\
\hline & & & & & $\begin{array}{c}\text { Diet }^{d} \\
(\mathbf{m g} / \mathbf{k g})\end{array}$ & $\begin{array}{l}\text { Watere } \\
(\mathrm{mg} / \mathrm{L})\end{array}$ & $\begin{array}{l}\text { Aquatic } \\
\text { Feeding } \\
\text { Species' } \\
\text { (mg/L) }\end{array}$ \\
\hline \multirow[t]{8}{*}{ Aroclor 1248} & $\begin{array}{l}\text { Rhesus } \\
\text { Monkey }\end{array}$ & 0.01 & $\begin{array}{l}\text { Short-tailed } \\
\text { Shrew }\end{array}$ & 0.068 & 0.113 & 0.309 & \\
\hline & & & Little Brown Bat & 0.085 & 0.256 & 0.534 & \\
\hline & & & $\begin{array}{c}\text { White-footed } \\
\text { Mouse }\end{array}$ & 0.060 & 0.388 & 0.200 & \\
\hline & & & Meadow Vole & 0.048 & 0.420 & 0.350 & \\
\hline & & & Cottontail Rabbit & 0.016 & 0.081 & 0.166 & \\
\hline & & & Mink & 0.017 & 0.124 & 0.172 & $1.41 \mathrm{e}-07$ \\
\hline & & & Red Fox & 0.010 & 0.104 & 0.123 & \\
\hline & & & Whitetail Deer & 0.004 & 0.146 & 0.069 & \\
\hline \multirow[t]{6}{*}{ Aroclor 1254} & $\begin{array}{l}\text { White-footed } \\
\text { mouse }\end{array}$ & 0.135 & $\begin{array}{c}\text { Short-tailed } \\
\text { Shrew }\end{array}$ & 0.148 & 0.247 & 0.675 & \\
\hline & & & Little Brown Bat & 0.187 & 0.560 & 1.166 & \\
\hline & & & $\begin{array}{c}\text { White-footed } \\
\text { Mouse }\end{array}$ & 0.131 & 0.846 & 0.436 & \\
\hline & & & Meadow Vole & 0.104 & 0.916 & 0.763 & \\
\hline & & & Cottontail Rabbit & 0.035 & 0.177 & 0.362 & \\
\hline & & & Whitetail Deer & 0.010 & 0.319 & 0.150 & \\
\hline \multirow[t]{2}{*}{ Aroclor 1254} & Mink & 0.137 & Mink & 0.137 & 1 & 1.384 & $4.33 e-07$ \\
\hline & & & Red Fox & 0.083 & 0.834 & 0.988 & \\
\hline
\end{tabular}


35

Table 12. (continued)

\begin{tabular}{|c|c|c|c|c|c|c|c|}
\hline \multirow{2}{*}{$\begin{array}{l}\text { Contaminant } \\
\text { and Form }\end{array}$} & \multirow{2}{*}{ Test Species } & \multirow{2}{*}{$\begin{array}{c}\text { Test } \\
\text { Species } \\
\text { NOAEL" } \\
(\mathbf{m g} / \mathbf{k g} \cdot \mathrm{d})\end{array}$} & \multirow{2}{*}{$\begin{array}{l}\text { Endpoint } \\
\text { Species }^{b}\end{array}$} & \multirow{2}{*}{$\begin{array}{l}\text { Estimated } \\
\text { Wildlife } \\
\text { NOAELc } \\
\text { (mg/kg • d) }\end{array}$} & \multicolumn{3}{|c|}{ Toxicological Benchmarks } \\
\hline & & & & & $\begin{array}{c}\text { Diet }^{d} \\
(\mathrm{mg} / \mathrm{kg})\end{array}$ & $\begin{array}{r}\text { Water } \\
\text { - (mg/L) }\end{array}$ & $\begin{array}{l}\text { Aquatic } \\
\text { Feeding } \\
\text { Species } \\
\text { (mg/L) }\end{array}$ \\
\hline \multirow[t]{9}{*}{ Aroclor 1254} & $\begin{array}{l}\text { Ring-necked } \\
\text { Pheasant }\end{array}$ & 0.18 & American Robin & 0.420 & 0.347 & 3.047 & \\
\hline & & & $\begin{array}{l}\text { American } \\
\text { Woodcock }\end{array}$ & 0.307 & 0.405 & 3.041 & \\
\hline & & & Wild Turkey & 0.101 & 3.359 & 3.076 & \\
\hline & & & $\begin{array}{c}\text { Belted } \\
\text { Kingfisher }\end{array}$ & 0.338 & 0.667 & 3.128 & $2.89 e-07$ \\
\hline & & & $\begin{array}{c}\text { Great Blue } \\
\text { Heron }\end{array}$ & 0.135 & 0.768 & 3.050 & $3.33 e-07$ \\
\hline & & & Barred Owl & 0.201 & 3.078 & 3.065 & \\
\hline & & & Barn Owl & 0.232 & 1.727 & 3.083 & \\
\hline & & & Cooper's Hawk & 0.236 & 3.050 & 3.050 & \\
\hline & & & Red-tailed Hawk & 0.173 & 0.214 & 3.045 & \\
\hline Arsenic & Mouse & 0.126 & $\begin{array}{l}\text { Short-tailed } \\
\text { Shrew }\end{array}$ & 0.158 & 0.264 & 0.720 & \\
\hline \multirow[t]{7}{*}{ Arsenite } & & & Little Brown Bat & 0.199 & 0.597 & 1.244 & \\
\hline & & & $\begin{array}{l}\text { White-footed } \\
\text { Mouse }\end{array}$ & 0.140 & 0.903 & 0.465 & \\
\hline & & & Meadow Vole & 0.111 & 0.977 & 0.814 & \\
\hline & & & Cottontail Rabbit & 0.037 & 0.189 & 0.386 & \\
\hline & & & Mink & 0.040 & 0.289 & 0.400 & $1.63 e-02$ \\
\hline & & & Red Fox & 0.024 & 0.241 & 0.286 & \\
\hline & & & Whitetail Deer & 0.010 & 0.340 & 0.160 & \\
\hline
\end{tabular}


36

Table 12. (continued)

\begin{tabular}{|c|c|c|c|c|c|c|c|}
\hline \multirow{2}{*}{$\begin{array}{l}\text { Contaminant } \\
\text { and Form }\end{array}$} & \multirow{2}{*}{ Test Species } & \multirow{2}{*}{$\begin{array}{c}\text { Test } \\
\text { Species } \\
\text { NOAEL" } \\
(\mathbf{m g} / \mathbf{k g} \cdot \mathbf{d})\end{array}$} & \multirow{2}{*}{$\begin{array}{l}\text { Endpoint } \\
\text { Species }^{b}\end{array}$} & \multirow{2}{*}{$\begin{array}{c}\text { Estimated } \\
\text { Wildlife } \\
\text { NOAELc } \\
(\mathrm{mg} / \mathrm{kg} \cdot \mathrm{d})\end{array}$} & \multicolumn{3}{|c|}{ Toxicological Benchmarks } \\
\hline & & & & & $\begin{array}{c}\text { Diet }^{d} \\
\text { (mg/kg) }\end{array}$ & $\begin{array}{l}\text { Watere } \\
\text { (mg/L). }\end{array}$ & $\begin{array}{l}\text { Aquatic } \\
\text { Feeding } \\
\text { Species }^{t} \\
\text { (mg/L) }\end{array}$ \\
\hline Arsenic & Mallard Duck & 5.135 & American Robin & 11.967 & 9.908 & 86.933 & \\
\hline \multirow[t]{8}{*}{ Sodium Arsenite } & & & $\begin{array}{l}\text { American } \\
\text { Woodcock }\end{array}$ & 8.763 & 11.567 & 86.751 & \\
\hline & & & Wild Turkey & 2.875 & 95.828 & 87.758 & \\
\hline & & & $\begin{array}{c}\text { Belted } \\
\text { Kingfisher }\end{array}$ & 9.646 & 19.035 & 89.227 & $1.11 e+00$ \\
\hline & & & $\begin{array}{c}\text { Great Blue } \\
\text { Heron }\end{array}$ & 3.852 & 21.919 & 87.013 & $1.27 e+\infty$ \\
\hline & & & Barred Owl & 5.731 & 87.800 & 87.426 & \\
\hline & & & Bam Owl & 6.607 & 49.258 & 87.961 & \\
\hline & & & Cooper's Hawk & 6.738 & 86.999 & 86.999 & \\
\hline & & & Red-tailed Hawk & 4.938 & 6.110 & 86.875 & \\
\hline Arsenic & $\begin{array}{l}\text { Brown-headed } \\
\text { Cowbird }\end{array}$ & 2.46 & American Robin & 2.119 & 1.755 & 15.394 & \\
\hline \multirow[t]{8}{*}{$\begin{array}{c}\text { Paris Green: } \\
\text { Copper Acetoarsenite }\end{array}$} & & & $\begin{array}{l}\text { American } \\
\text { Woodcock }\end{array}$ & 1.552 & 2.048 & 15.362 & \\
\hline & & & Wild Turkey & 0.509 & 16.968 & 15.539 & \\
\hline & & & $\begin{array}{c}\text { Belted } \\
\text { Kingfisher }\end{array}$ & 1.708 & 3.371 & 15.800 & $1.96 \mathrm{e}-01$ \\
\hline & & & $\begin{array}{c}\text { Great Blue } \\
\text { Heron }\end{array}$ & 0.682 & 3.881 & 15.408 & $2.25 \mathrm{e}-01$ \\
\hline & & & Barred Owl & 1.015 & 15.547 & 15.481 & \\
\hline & & & Barn Owl & 1.170 & 8.722 & 15.576 & \\
\hline & & & Cooper's Hawk & 1.193 & 15.405 & 15.405 & \\
\hline & & & Red-tailed Hawk & 0.874 & 1.082 & 15.383 & \\
\hline
\end{tabular}


37

Table 12. (continued)

\begin{tabular}{|c|c|c|c|c|c|c|c|}
\hline \multirow{2}{*}{$\begin{array}{l}\text { Contaminant } \\
\text { and Form }\end{array}$} & \multirow{2}{*}{ Test Species } & \multirow{2}{*}{$\begin{array}{c}\text { Test } \\
\text { Species } \\
\text { NOAEL" } \\
\left(\mathrm{mg} / \mathbf{k g} \bullet^{\circ} \mathrm{d}\right)\end{array}$} & \multirow{2}{*}{$\begin{array}{l}\text { Endpoint } \\
\text { Species }^{b}\end{array}$} & \multirow{2}{*}{$\begin{array}{c}\text { Estimated } \\
\text { Wildlife } \\
\text { NOAEL } \\
\left(\mathrm{mg} / \mathrm{kg}^{\circ} \cdot \mathrm{d}\right)\end{array}$} & \multicolumn{3}{|c|}{ Toxicological Benchmarks } \\
\hline & & & & & $\begin{array}{c}\text { Diet }^{d} \\
(\mathbf{m g} / \mathbf{k g})\end{array}$ & $\begin{array}{r}\text { Water } \\
\text {. }(\mathrm{mg} / \mathrm{L})\end{array}$ & $\begin{array}{l}\text { Aquatic } \\
\text { Feeding } \\
\text { Species } \\
\text { (mg/L) }\end{array}$ \\
\hline Barium & Rat & 5.06 & $\begin{array}{l}\text { Short-tailed } \\
\text { Shrew }\end{array}$ & 15.372 & 25.621 & 69.874 & \\
\hline \multirow[t]{7}{*}{ Braium Chloride } & & & Little Brown Bat & 19.323 & 57.970 & 120.771 & \\
\hline & & & $\begin{array}{c}\text { White-footed } \\
\text { Mouse }\end{array}$ & 13.547 & 87.659 & 45.158 & \\
\hline & & & Meadow Vole & 10.777 & 94.841 & 79.034 & \\
\hline & & & Cottontail Rabbit & 3.620 & 18.330 & 37.450 & \\
\hline & & & Mink & 3.845 & 28.063 & 38.834 & \\
\hline & & & Red Fox & 2.340 & 23.404 & 27.715 & \\
\hline & & & Whitetail Deer & 1.015 & 32.974 & 15.507 & \\
\hline Barium & Chicken & 20.86 & American Robin & 24.215 & 20.049 & 175.904 & \\
\hline \multirow[t]{8}{*}{ Barium Hydroxide } & & & $\begin{array}{l}\text { American } \\
\text { Woodcock }\end{array}$ & 17.731 & 23.405 & 175.537 & \\
\hline & & & Wild Turkey & 5.817 & 193.901 & 177.572 & \\
\hline & & & $\begin{array}{c}\text { Belted } \\
\text { Kingfisher }\end{array}$ & 19.518 & 38.516 & 180.546 & \\
\hline & & & $\begin{array}{c}\text { Great Blue } \\
\text { Heron }\end{array}$ & 7.794 & 44.352 & 176.068 & \\
\hline & & & Barred Owl & 11.596 & 177.658 & 176.902 & \\
\hline & & & Barn Owl & 13.368 & 99.671 & 177.984 & \\
\hline & & & Cooper's Hawk & 13.634 & 176.038 & 176.038 & \\
\hline & & & Red-tailed Hawk & 9.991 & 12.363 & 175.785 & \\
\hline
\end{tabular}


38

Table 12. (continued)

\begin{tabular}{|c|c|c|c|c|c|c|c|}
\hline \multirow{2}{*}{$\begin{array}{l}\text { Contaminant } \\
\text { and Form }\end{array}$} & \multirow{2}{*}{ Test Species } & \multirow{2}{*}{$\begin{array}{c}\text { Test } \\
\text { Species } \\
\text { NOAEL" } \\
(\mathbf{m g} / \mathbf{k g} \cdot \mathbf{d})\end{array}$} & \multirow{2}{*}{$\begin{array}{l}\text { Endpoint } \\
\text { Species }\end{array}$} & \multirow{2}{*}{$\begin{array}{c}\text { Estimated } \\
\text { Wildlife } \\
\text { NOAELc } \\
(\mathrm{mg} / \mathrm{kg} \bullet \mathrm{d})\end{array}$} & \multicolumn{3}{|c|}{ Toxicological Benchmarks } \\
\hline & & & & & $\begin{array}{c}\text { Diet }^{d} \\
(\mathrm{mg} / \mathrm{kg})\end{array}$ & $\begin{array}{l}\text { Watere } \\
\text { (mg/L) }\end{array}$ & $\begin{array}{l}\text { Aquatic } \\
\text { Feeding } \\
\text { Speciest } \\
\text { (mg/L) }\end{array}$ \\
\hline \multirow[t]{8}{*}{ Benzene } & Mouse & 26.36 & $\begin{array}{l}\text { Short-tailed } \\
\text { Shrew }\end{array}$ & 33.135 & 55.225 & 150.613 & \\
\hline & & & Little Brown Bat & 41.651 & 124.953 & 260.318 & \\
\hline & & & $\begin{array}{c}\text { White-footed } \\
\text { Mouse }\end{array}$ & 29.201 & 188.946 & 97.336 & . \\
\hline & & & Meadow Vole & 23.230 & 204.426 & 170.355 & \\
\hline & & & Cottontail Rabbit & 7.803 & 39.509 & 80.722 & \\
\hline & & & Mink & 8.287 & 60.489 & 83.708 & $2.40 e+00$ \\
\hline & & & Red Fox & 5.045 & 50.448 & 59.741 & \\
\hline & & & Whitetail Deer & 2.189 & 71.077 & 33.426 & \\
\hline \multirow[t]{8}{*}{ beta-BHC } & Rat & 0.4 & $\begin{array}{l}\text { Short-tailed } \\
\text { Shrew }\end{array}$ & 1.131 & 1.885 & 5.141 & \\
\hline & & & Little Brown Bat & 1.422 & 4.265 & 8.886 & \\
\hline & & & $\begin{array}{l}\text { White-footed } \\
\text { Mouse }\end{array}$ & 0.997 & 6.450 & 3.323 & \\
\hline & & & Meadow Vole & 0.793 & 6.978 & 5.815 & \\
\hline & & & Cottontail Rabbit & 0.266 & 1.349 & 2.755 & \\
\hline & & & Mink & 0.283 & 2.065 & 2.857 & \\
\hline & & & Red Fox & 0.172 & 1.722 & 2.039 & \\
\hline & & & Whitetail Deer & 0.075 & 2.426 & 1.141 & \\
\hline
\end{tabular}


39

Table 12. (continued)

\begin{tabular}{|c|c|c|c|c|c|c|c|}
\hline \multirow{2}{*}{$\begin{array}{l}\text { Contaminant } \\
\text { and Form }\end{array}$} & \multirow{2}{*}{ Test Species } & \multirow{2}{*}{$\begin{array}{c}\text { Test } \\
\text { Species } \\
\text { NOAEL" } \\
(\mathbf{m g} / \mathbf{k g} \bullet \mathbf{d})\end{array}$} & \multirow{2}{*}{$\begin{array}{l}\text { Endpoint } \\
\text { Species }\end{array}$} & \multirow{2}{*}{$\begin{array}{c}\text { Estimated } \\
\text { Wildlife } \\
\text { NOAELc } \\
\left(\mathrm{mg} / \mathrm{kg}^{\bullet} \mathrm{d}\right)\end{array}$} & \multicolumn{3}{|c|}{ Toxicological Benchmarks } \\
\hline & & & & & $\begin{array}{c}\text { Diet }^{d} \\
(\mathrm{mg} / \mathrm{kg})\end{array}$ & $\begin{array}{l}\text { Watere } \\
\text { - (mg/L) }\end{array}$ & $\begin{array}{c}\text { Aquatic } \\
\text { Feeding } \\
\text { Species } \\
\text { (mg/L) }\end{array}$ \\
\hline \multirow[t]{6}{*}{ BHC-mixed isomers } & Rat & 1.6 & $\begin{array}{l}\text { Short-tailed } \\
\text { Shrew }\end{array}$ & 4.524 & 7.541 & 20.565 & \\
\hline & & & Little Brown Bat & 5.687 & 17.061 & 35.545 & \\
\hline & & & $\begin{array}{l}\text { White-footed } \\
\text { Mouse }\end{array}$ & 3.987 & 25.799 & 13.291 & \\
\hline & & & Meadow Vole & 3.172 & 27.913 & 23.261 & \\
\hline & & & Cottontail Rabbit & 1.065 & 5.395 & 11.022 & \\
\hline & & & Whitetail Deer & 0.299 & 9.705 & 4.564 & \\
\hline \multirow[t]{2}{*}{ BHC-mixed isomers } & Mink & 0.0137 & Mink & 0.014 & 0.100 & 0.138 & $4.23 e-06$ \\
\hline & & & Red Fox & 0.008 & 0.083 & 0.099 & \\
\hline \multirow[t]{9}{*}{ BHC-mixed isomers } & $\begin{array}{l}\text { Japanese } \\
\text { Quail }\end{array}$ & 0.563 & American Robin & 0.702 & 0.581 & 5.096 & \\
\hline & & & $\begin{array}{c}\text { American } \\
\text { Woodcock }\end{array}$ & 0.514 & 0.678 & 5.086 & \\
\hline & & & Wild Turkey & 0.169 & 5.618 & 5.145 & \\
\hline & & & $\begin{array}{c}\text { Belted } \\
\text { Kingfisher }\end{array}$ & 0.566 & 1.116 & 5.231 & $4.72 e-05$ \\
\hline & & & $\begin{array}{c}\text { Great Blue } \\
\text { Heron }\end{array}$ & 0.226 & 1.285 & 5.101 & $5.43 e-05$ \\
\hline & & & Barred Owl & 0.336 & 5.147 & 5.125 & \\
\hline & & & Barn Owl & 0.387 & 2.888 & 5.157 & \\
\hline & & & Cooper's Hawk & 0.395 & 5.100 & 5.100 & \\
\hline & & & Red-tailed Hawk & 0.289 & 0.358 & 5.093 & \\
\hline
\end{tabular}


40

Table 12. (continued)

\begin{tabular}{|c|c|c|c|c|c|c|c|}
\hline \multirow[b]{2}{*}{$\begin{array}{l}\text { Contaminant } \\
\text { and Form }\end{array}$} & \multirow{2}{*}{ Test Species } & \multirow{2}{*}{$\begin{array}{c}\text { Test } \\
\text { Species } \\
\text { NOAEL" } \\
\left(\mathbf{m g} / \mathrm{kg}^{\circ} \mathrm{d}\right)\end{array}$} & \multirow{2}{*}{$\begin{array}{l}\text { Endpoint } \\
\text { Species }^{b}\end{array}$} & \multirow{2}{*}{$\begin{array}{c}\text { Estimated } \\
\text { Wildlife } \\
\text { NOAEL } \\
(\mathrm{mg} / \mathrm{kg} \cdot \mathrm{d})\end{array}$} & \multicolumn{3}{|c|}{ Toxicological Benchmarks } \\
\hline & & & & & $\underset{\text { (mg/kg) }}{\text { Diet }^{d}}$ & $\begin{array}{l}\text { Water } \\
\text { (mg/L) }\end{array}$ & $\begin{array}{l}\text { Aquatic } \\
\text { Feeding } \\
\text { Species } \\
\text { (mg/L) }\end{array}$ \\
\hline \multirow[t]{8}{*}{ Benzo(a)pyrene } & Mouse & 1 & $\begin{array}{l}\text { Short-tailed } \\
\text { Shrew }\end{array}$ & 1.257 & 2.095 & 5.714 & \\
\hline & & & Little Brown Bat & 1.580 & 4.740 & 9.876 & \\
\hline & & & $\begin{array}{l}\text { White-footed } \\
\text { Mouse }\end{array}$ & 1.108 & 7.168 & 3.693 & \\
\hline & & & Meadow Vole & 0.881 & 7.755 & 6.463 & \\
\hline & & & Cottontail Rabbit & 0.296 & 1.499 & 3.062 & \\
\hline & & & Mink & 0.314 & 2.295 & 3.176 & $3.60 \mathrm{e}-06$ \\
\hline & . & & Red Fox & 0.191 & 1.914 & 2.266 & \\
\hline & & & Whitetail Deer & 0.083 & 2.696 & 1.268 & \\
\hline Beryllium & Rat & 0.66 & $\begin{array}{c}\text { Short-tailed } \\
\text { Shrew }\end{array}$ & 1.866 & 3.110 & 8.483 & \\
\hline \multirow[t]{7}{*}{ Beryllium Sulfate } & & & Little Brown Bat & 2.346 & 7.038 & 14.662 & \\
\hline & & & $\begin{array}{c}\text { White-footed } \\
\text { Mouse }\end{array}$ & 1.645 & 10.642 & 5.482 & \\
\hline & & & Meadow Vole & 1.308 & 11.514 & 9.595 & \\
\hline & & & Cottontail Rabbit & 0.440 & 2.225 & 4.547 & \\
\hline & & & Mink & 0.467 & 3.407 & 4.715 & $1.73 \mathrm{e}-01$ \\
\hline & & & Red Fox & 0.284 & 2.841 & 3.365 & \\
\hline & & & Whitetail Deer & 0.123 & 4.003 & 1.883 & \\
\hline
\end{tabular}


41

Table 12. (continued)

\begin{tabular}{|c|c|c|c|c|c|c|c|}
\hline \multirow{2}{*}{$\begin{array}{l}\text { Contaminant } \\
\text { and Form }\end{array}$} & \multirow{2}{*}{ Test Species } & \multirow{2}{*}{$\begin{array}{c}\text { Test } \\
\text { Species } \\
\text { NOAEL' } \\
(\mathbf{m g} / \mathbf{k g} \cdot \mathbf{d})\end{array}$} & \multirow{2}{*}{$\begin{array}{l}\text { Endpoint } \\
\text { Species }^{\mathrm{b}}\end{array}$} & \multirow{2}{*}{$\begin{array}{c}\text { Estimated } \\
\text { Wildilife } \\
\text { NOAEL } \\
\left(\mathrm{mg} / \mathrm{kg}^{\circ} \mathrm{d}\right)\end{array}$} & \multicolumn{3}{|c|}{ Toxicological Benchmarks } \\
\hline & & & & & $\begin{array}{c}\text { Diet }^{d} \\
(\mathrm{mg} / \mathrm{kg})\end{array}$ & $\begin{array}{l}\text { Water } \\
\text { (mg/L) }\end{array}$ & $\begin{array}{l}\text { Aquatic } \\
\text { Feeding } \\
\text { Species } \\
\text { (mg/L) }\end{array}$ \\
\hline \multirow[t]{8}{*}{$\begin{array}{l}\text { Bis(2-ethylhexyl) } \\
\text { phthalate }\end{array}$} & mouse & 18.33 & $\begin{array}{l}\text { Short-tailed } \\
\text { Shrew }\end{array}$ & 23.041 & 38.402 & 104.732 & \\
\hline & & & Little Brown Bat & 28.963 & 86.889 & 181.018 & \\
\hline & & & $\begin{array}{l}\text { White-footed } \\
\text { Mouse }\end{array}$ & 20.305 & 131.388 & 67.685 & \\
\hline & & & Meadow Vole & 16.154 & 142.152 & 118.460 & \\
\hline & & & Cottontail Rabbit & 5.426 & 27.474 & 56.132 & \\
\hline & & & Mink & 5.763 & 42.063 & 58.208 & $3.74 e-03$ \\
\hline & & & Red Fox & 3.508 & 35.080 & 41.542 & \\
\hline & & & Whitetail Deer & 1.522 & 49.425 & 23.243 & \\
\hline \multirow{9}{*}{$\begin{array}{l}\text { Bis(2-ethylhexyl) } \\
\text { phthalate }\end{array}$} & ringed dove & 1.11 & American Robin & 1.398 & 1.158 & 10.157 & \\
\hline & & & $\begin{array}{l}\text { American } \\
\text { Woodcock }\end{array}$ & 1.024 & 1.351 & 10.136 & \\
\hline & & & Wild Turkey & 0.336 & 11.197 & 10.254 & \\
\hline & & & $\begin{array}{c}\text { Belted } \\
\text { Kingfisher }\end{array}$ & 1.127 & 2.224 & 10.425 & $1.98 \mathrm{e}-04$ \\
\hline & & & $\begin{array}{c}\text { Great Blue } \\
\text { Heron }\end{array}$ & 0.450 & 2.561 & 10.167 & $2.27 e-04$ \\
\hline & & & Barred Owl & 0.670 & 10.259 & 10.215 & \\
\hline & & & Barn Owl & 0.772 & 5.755 & 10.277 & \\
\hline & & & Cooper's Hawk & 0.787 & 10.165 & 10.165 & \\
\hline & & & Red-tailed Hawk & 0.577 & 0.714 & 10.150 & \\
\hline
\end{tabular}


42

Table 12. (continued)

\begin{tabular}{|c|c|c|c|c|c|c|c|}
\hline \multirow[b]{2}{*}{$\begin{array}{l}\text { Contaminant } \\
\text { and Form }\end{array}$} & \multirow{2}{*}{ Test Species } & \multirow{2}{*}{$\begin{array}{c}\text { Test } \\
\text { Species } \\
\text { NOAEL" } \\
(\mathrm{mg} / \mathrm{kg} \bullet \mathrm{d})\end{array}$} & \multirow{2}{*}{$\begin{array}{l}\text { Endpoint } \\
\text { Species }^{b}\end{array}$} & \multirow{2}{*}{$\begin{array}{c}\text { Estimated } \\
\text { Wildlife } \\
\text { NOAEL } \\
\left(\mathrm{mg} / \mathrm{kg}^{\bullet} \mathrm{d}\right)\end{array}$} & \multicolumn{3}{|c|}{ Toxicological Benchmarks } \\
\hline & & & & & $\begin{array}{c}\text { Diet }^{d} \\
(\mathrm{mg} / \mathrm{kg})\end{array}$ & $\begin{array}{l}\text { Watere } \\
\text { (mg/L) }\end{array}$ & $\begin{array}{l}\text { Aquatic } \\
\text { Feeding } \\
\text { Speciest } \\
\text { (mg/L) }\end{array}$ \\
\hline Cadmium & mouse & 0.1913 & $\begin{array}{l}\text { Shorr-tailed } \\
\text { Shrew }\end{array}$ & 0.240 & 0.401 & 1.093 & \\
\hline \multirow[t]{7}{*}{ Soluble salt } & & & Litule Brown Bat & 0.302 & 0.907 & 1.889 & \\
\hline & & & $\begin{array}{l}\text { White-footed } \\
\text { Mouse }\end{array}$ & 0.212 & 1.371 & 0.706 & \\
\hline & & & Meadow Vole & 0.169 & 1.484 & 1.236 & \\
\hline & & & Cottontail Rabbit & 0.057 & 0.287 & 0.586 & \\
\hline & & & Mink & 0.060 & 0.439 & 0.607 & $3.54 e-05$ \\
\hline & & & Red Fox & 0.037 & 0.366 & 0.434 & \\
\hline & & & Whitetail Deer & 0.016 & 0.516 & 0.243 & \\
\hline Cadmium & mallard duck & 1.45 & American Robin & 3.542 & 2.932 & 25.728 & \\
\hline \multirow[t]{8}{*}{ Cadmium Chloride } & & & $\begin{array}{l}\text { American } \\
\text { Woodcock }\end{array}$ & 2.593 & 3.423 & 25.675 & \\
\hline & & & Wild Turikey & 0.851 & 28.361 & 25.973 & \\
\hline & & & $\begin{array}{c}\text { Belted } \\
\text { Kingfisher }\end{array}$ & 2.855 & 5.634 & 26.407 & $4.54 \mathrm{e}-04$ \\
\hline & & & $\begin{array}{c}\text { Great Blue } \\
\text { Heron }\end{array}$ & 1.140 & 6.487 & 25.752 & $5.23 e-04$ \\
\hline & & & Barred Owl & 1.696 & 25.985 & 25.874 & \\
\hline & & & Bam Owl & 1.955 & 14.578 & 26.033 & \\
\hline & & & Cooper's Hawk & 1.994 & 25.748 & 25.748 & \\
\hline & & & Red-tailed Hawk & 1.461 & 1.808 & 25.711 & \\
\hline
\end{tabular}


43

Table 12. (continued)

\begin{tabular}{|c|c|c|c|c|c|c|c|}
\hline \multirow{2}{*}{$\begin{array}{l}\text { Contaminant } \\
\text { and Form }\end{array}$} & \multirow{2}{*}{ Test Species } & \multirow{2}{*}{$\begin{array}{c}\text { Test } \\
\text { Species } \\
\text { NOAEL" } \\
(\mathbf{m g} / \mathbf{k g} \cdot \mathrm{d})\end{array}$} & \multirow{2}{*}{$\begin{array}{l}\text { Endpoint } \\
\text { Species }^{b}\end{array}$} & \multirow{2}{*}{$\begin{array}{c}\text { Estimated } \\
\text { Wildlife } \\
\text { NOAEL } \\
\left(\mathrm{mg} / \mathrm{kg}^{\cdot} \cdot \mathrm{d}\right)\end{array}$} & \multicolumn{3}{|c|}{ Toxicological Benchmarks } \\
\hline & & & & & $\begin{array}{c}\text { Diet }^{d} \\
(\mathrm{mg} / \mathbf{k g})\end{array}$ & $\begin{array}{r}\text { Watere } \\
\text { - (mg/L) }\end{array}$ & $\begin{array}{l}\text { Aquatic } \\
\text { Feeding } \\
\text { Species } \\
\text { (mg/L) }\end{array}$ \\
\hline \multirow[t]{8}{*}{ Carbon Tetrachloride } & Rat & 16 & $\begin{array}{c}\text { Short-tailed } \\
\text { Shrew }\end{array}$ & 45.243 & 75.405 & 205.650 & \\
\hline & & & Little Brown Bat & 56.871 & 170.614 & 355.445 & \\
\hline & & & $\begin{array}{l}\text { White-footed } \\
\text { Mouse }\end{array}$ & 39.872 & 257.992 & 132.905 & \\
\hline & & & Meadow Vole & 31.719 & 279.129 & 232.607 & \\
\hline & & & Cottontail Rabbit & 10.655 & 53.947 & 110.220 & \\
\hline & & & Mink & 11.315 & 82.593 & 114.295 & $9.83 e-01$ \\
\hline & & & Red Fox & 6.888 & 68.882 & 81.570 & \\
\hline & & & Whitetail Deer & 2.989 & 97.050 & 45.640 & \\
\hline \multirow[t]{8}{*}{ Chlordane } & mouse & 4.58 & $\begin{array}{l}\text { Short-tailed } \\
\text { Shrew }\end{array}$ & 5.757 & 9.595 & 26.169 & \\
\hline & & & Little Brown Bat & 7.237 & 21.710 & 45.230 & \\
\hline & & & $\begin{array}{l}\text { White-footed } \\
\text { Mouse }\end{array}$ & 5.074 & 32.829 & 16.912 & \\
\hline & & & Meadow Vole & 4.036 & 35.519 & 29.599 & \\
\hline & & & Cottontail Rabbit & 1.356 & 6.865 & 14.025 & \\
\hline & & & Mink & 1.440 & 10.510 & 14.544 & $1.86 \mathrm{e}-04$ \\
\hline & & & Red Fox & 0.877 & 8.765 & 10.380 & \\
\hline & & & Whitetail Deer & 0.380 & 12.349 & 5.808 & \\
\hline
\end{tabular}


44

Table 12. (continued)

\begin{tabular}{|c|c|c|c|c|c|c|c|}
\hline \multirow[b]{2}{*}{$\begin{array}{l}\text { Contaminant } \\
\text { and Form }\end{array}$} & \multirow{2}{*}{ Test Species } & \multirow{2}{*}{$\begin{array}{c}\text { Test } \\
\text { Species } \\
\text { NOAEL: } \\
\left(\mathrm{mg} / \mathrm{kg}^{\circ} \mathbf{d}\right)\end{array}$} & \multirow{2}{*}{$\begin{array}{l}\text { Endpoint } \\
\text { Species }^{b}\end{array}$} & \multirow{2}{*}{$\begin{array}{c}\text { Estimated } \\
\text { Wildlife } \\
\text { NOAEL } \\
(\mathrm{mg} / \mathrm{kg} \cdot \mathrm{d})\end{array}$} & \multicolumn{3}{|c|}{ Toxicological Benchmarks } \\
\hline & & & & & $\begin{array}{c}\text { Diet }^{d} \\
\text { (mg/kg) }\end{array}$ & $\begin{array}{l}\text { Water } \\
\text { (mg/L) }\end{array}$ & $\begin{array}{l}\text { Aquatic } \\
\text { Feeding } \\
\text { Species? } \\
\text { (mg/L) }\end{array}$ \\
\hline \multirow[t]{9}{*}{ Chlondane } & $\begin{array}{l}\text { red-winged } \\
\text { blackbird }\end{array}$ & 2.14 & American Robin & 2.013 & 1.667 & 14.625 & \\
\hline & & & $\begin{array}{l}\text { American } \\
\text { Woodcock }\end{array}$ & 1.474 & 1.946 & 14.594 & \\
\hline & & & Wild Turkey & 0.484 & 16.121 & 14.764 & \\
\hline & & & $\begin{array}{c}\text { Belted } \\
\text { Kingfisher }\end{array}$ & 1.623 & 3.202 & 15.011 & $5.68 \mathrm{e}-05$ \\
\hline & & & $\begin{array}{c}\text { Great Blue } \\
\text { Heron }\end{array}$ & 0.648 & 3.687 & 14.638 & $6.54 \mathrm{e}-05$ \\
\hline & & & Barred Owl & 0.964 & 14.771 & 14.708 & \\
\hline & & & Barn Owl & 1.111 & 8.287 & 14.798 & \\
\hline & & & Cooper's Hawk & 1.134 & 14.636 & 14.636 & \\
\hline & & & Red-tailed Hawk & 0.831 & 1.028 & 14.615 & \\
\hline \multirow[t]{8}{*}{ Chlordecone (Kepone) } & Rat & 0.08 & $\begin{array}{c}\text { Short-tailed } \\
\text { Shrew }\end{array}$ & 0.226 & 0.377 & 1.028 & \\
\hline & & & Litule Brown Bat & 0.284 & 0.853 & 1.777 & \\
\hline & & & $\begin{array}{l}\text { White-footed } \\
\text { Mouse }\end{array}$ & 0.199 & 1.290 & 0.665 & \\
\hline & & & Meadow Vole & 0.159 & 1.396 & 1.163 & \\
\hline & & & Cottontail Rabbit & 0.053 & 0.270 & 0.551 & \\
\hline & & : & Mink & 0.057 & 0.413 & 0.572 & \\
\hline & & & Red Fox & 0.034 & 0.344 & 0.408 & \\
\hline & & & Whitetail Deer & 0.015 & 0.485 & 0.228 & \\
\hline
\end{tabular}


45

Table 12. (continued)

\begin{tabular}{|c|c|c|c|c|c|c|c|}
\hline \multirow{2}{*}{$\begin{array}{c}\text { Contaminant } \\
\text { and Form }\end{array}$} & \multirow{2}{*}{ Test Species } & \multirow{2}{*}{$\begin{array}{c}\text { Test } \\
\text { Species } \\
\text { NOAEL" } \\
\left(\mathbf{m g} / \mathbf{k g}^{*} \mathbf{d}\right)\end{array}$} & \multirow{2}{*}{$\begin{array}{l}\text { Endpoint } \\
\text { Species }\end{array}$} & \multirow{2}{*}{$\begin{array}{c}\text { Estimated } \\
\text { Wildlife } \\
\text { NOAELc } \\
(\mathbf{m g} / \mathbf{k g} \cdot \mathbf{d})\end{array}$} & \multicolumn{3}{|c|}{ Toxicological Benchmarks } \\
\hline & & & & & $\begin{array}{c}\text { Diet }^{\mathrm{d}} \\
\text { (mg/kg) }\end{array}$ & $\cdot \begin{array}{c}\text { Water } \\
(\mathrm{mg} / \mathrm{L})\end{array}$ & $\begin{array}{l}\text { Aquatic } \\
\text { Feeding } \\
\text { Species } \\
\text { (mg/L) }\end{array}$ \\
\hline \multirow[t]{8}{*}{ Chloroform } & Rat & 15 & $\begin{array}{c}\text { Short-tailed } \\
\text { Shrew }\end{array}$ & 42.415 & 70.692 & 192.797 & \\
\hline & & & Litule Brown Bat & 53.317 & 159.950 & 333.230 & \\
\hline & & & $\begin{array}{l}\text { White-footed } \\
\text { Mouse }\end{array}$ & 37.380 & 241.868 & 124.599 & \\
\hline & & & Meadow Vole & 29.737 & 261.683 & 218.070 & \\
\hline & & & Cottontail Rabbit & 9.989 & 50.575 & 103.331 & \\
\hline & & & Mink & 10.608 & 77.431 & 107.152 & $4.03 e+\infty$ \\
\hline & & & Red Fox & 6.458 & 64.577 & 76.472 & \\
\hline & & & Whitetail Deer & 2.802 & 90.984 & 42.787 & \\
\hline Chromium & Rat & 2737 & $\begin{array}{c}\text { Short-tailed } \\
\text { Shrew }\end{array}$ & 7739.388 & 12898.979 & 35179.034 & \\
\hline \multirow[t]{7}{*}{$\mathrm{Cr}+3$ as $\mathrm{Cr} 2 \mathrm{O} 3$} & & & Little Brown Bat & 9728.530 & 29185.589 & 60803.310 & \\
\hline & & & $\begin{array}{l}\text { White-footed } \\
\text { Mouse }\end{array}$ & 6820.522 & 44132.789 & 22735.073 & \\
\hline & & & Meadow Vole & 5425.966 & 47748.498 & 39790.415 & \\
\hline & & & Cottontail Rabbit & 1822.596 & 9228.333 & 18854.438 & \\
\hline & & & Mink & 1935.606 & 14128.514 & 19551.580 & \\
\hline & & & Red Fox & 1178.306 & 11783.059 & 13953.622 & \\
\hline & & & Whitetail Deer & 511.272 & 16601.635 & 7807.256 & \\
\hline
\end{tabular}


46

Table 12. (continued)

\begin{tabular}{|c|c|c|c|c|c|c|c|}
\hline \multirow[b]{2}{*}{$\begin{array}{l}\text { Contaminant } \\
\text { and Form }\end{array}$} & \multirow{2}{*}{ Test Species } & \multirow{2}{*}{$\begin{array}{c}\text { Test } \\
\text { Species } \\
\text { NOAEL } \\
(\mathbf{m g} / \mathbf{k g} \cdot \mathbf{d})\end{array}$} & \multirow{2}{*}{$\begin{array}{l}\text { Endpoint } \\
\text { Species }^{b}\end{array}$} & \multirow{2}{*}{$\begin{array}{c}\text { Estimated } \\
\text { Wildlife } \\
\text { NOAEL } \\
(\mathrm{mg} / \mathbf{k g} \bullet \mathbf{d})\end{array}$} & \multicolumn{3}{|c|}{ Toxicological Benchmarks } \\
\hline & & & & & $\begin{array}{c}\text { Diet }^{d} \\
\text { (mg/kg) }\end{array}$ & $\begin{array}{l}\text { Watere } \\
(\mathbf{m g} / \mathbf{L})\end{array}$ & $\begin{array}{l}\text { Aquatic } \\
\text { Feeding } \\
\text { Species } \\
\text { (mg/L) }\end{array}$ \\
\hline Chromium & black duck & 1 & American Robin & 2.509 & 2.077 & 18.223 & \\
\hline \multirow[t]{8}{*}{$\mathrm{Cr}+3$ as $\mathrm{CrK}(\mathrm{SO} 4) 2$} & & & $\begin{array}{l}\text { American } \\
\text { Woodcock }\end{array}$ & 1.837 & 2.425 & 18.185 & \\
\hline & & & Wild Turkey & 0.603 & 20.088 & 18.396 & \\
\hline & & & $\begin{array}{c}\text { Belted } \\
\text { Kingfisher }\end{array}$ & 2.022 & 3.990 & 18.704 & \\
\hline & & & $\begin{array}{c}\text { Great Blue } \\
\text { Heron }\end{array}$ & 0.807 & 4.595 & 18.240 & \\
\hline & & & Barred Owl & 1.201 & 18.405 & 18.327 & \\
\hline & & & Barn Owl & 1.385 & 10.326 & 18.439 & \\
\hline & & & Cooper's Hawk & 1.412 & 18.237 & 18.237 & \\
\hline & & & Red-tailed Hawk & 1.035 & 1.281 & 18.211 & \\
\hline Chromium & Rat & 3.28 & $\begin{array}{c}\text { Short-tailed } \\
\text { Shrew }\end{array}$ & 9.275 & 15.458 & 42.158 & \\
\hline \multirow[t]{7}{*}{$\mathrm{Cr}+6$ as $\mathrm{K} 2 \mathrm{Cr} 2 \mathrm{O} 4$} & & & Little Brown Bat & 11.659 & 34.976 & 72.866 & \\
\hline & & & $\begin{array}{c}\text { White-footed } \\
\text { Mouse }\end{array}$ & 8.174 & 52.888 & 27.246 & \\
\hline & & & Meadow Vole & 6.502 & 57.221 & -47.685 & \\
\hline & & & Cottontail Rabbit & 2.184 & 11.059 & 22.595 & \\
\hline & & & Mink & 2.320 & 16.932 & 23.431 & $4.55 e+00$ \\
\hline & & & Red Fox & 1.412 & 14.121 & 16.722 & \\
\hline & & & Whitetail Deer & 0.613 & 19.895 & 9.356 & \\
\hline
\end{tabular}


47

Table 12. (continued)

\begin{tabular}{|c|c|c|c|c|c|c|c|}
\hline \multirow{2}{*}{$\begin{array}{l}\text { Contaminant } \\
\text { and Form }\end{array}$} & \multirow{2}{*}{ Test Species } & \multirow{2}{*}{$\begin{array}{c}\text { Test } \\
\text { Species } \\
\text { NOAEL" } \\
\left(\mathrm{mg} / \mathrm{kg} \bullet^{\circ} \mathrm{d}\right)\end{array}$} & \multirow{2}{*}{$\begin{array}{l}\text { Endpoint } \\
\text { Species' }\end{array}$} & \multirow{2}{*}{$\begin{array}{c}\text { Estimated } \\
\text { Wildife } \\
\text { NOAEL } \\
\left(\mathrm{mg} / \mathrm{kg}^{\bullet} \mathrm{d}\right)\end{array}$} & \multicolumn{3}{|c|}{ Toxicological Benchmarks } \\
\hline & & & & & $\begin{array}{c}\text { Diet }^{d} \\
\text { (mg/kg) }\end{array}$ & $\begin{array}{l}\text { Water }{ }^{e} \\
(\mathrm{mg} / \mathrm{L})\end{array}$ & $\begin{array}{l}\text { Aquatic } \\
\text { Feeding } \\
\text { Species } \\
\text { (mg/L) }\end{array}$ \\
\hline Copper & Mink & 11.71 & $\begin{array}{l}\text { Short-tailed } \\
\text { Shrew }\end{array}$ & 46.822 & 78.036 & 212.826 & \\
\hline \multirow[t]{7}{*}{ Copper Sulfate } & & & Litule Brown Bat & 58.855 & 176.566 & 367.846 & \\
\hline & & & $\begin{array}{l}\text { White-footed } \\
\text { Mouse }\end{array}$ & 41.263 & 266.994 & 137.542 & \\
\hline & & & Meadow Vole & 32.826 & 288.868 & 240.724 & \\
\hline & & & Cottontail Rabbit & 11.026 & 55.829 & 114.065 & \\
\hline & & & Mink & 11.710 & 85.474 & 118.283 & $2.94 \mathrm{e}-01$ \\
\hline & & & Red Fox & 7.128 & 71.285 & 84.416 & \\
\hline & & & Whitetail Deer & 3.093 & 100.432 & 47.230 & \\
\hline Copper & Chicken & 33.21 & American Robin & 62.924 & 52.098 & 457.089 & \\
\hline \multirow[t]{8}{*}{ Copper Oxide } & & & $\begin{array}{l}\text { American } \\
\text { Woodcock }\end{array}$ & 46.074 & 60.818 & 456.135 & \\
\hline & & & Wild Turkey & 15.116 & 503.851 & 461.421 & \\
\hline & & & $\begin{array}{c}\text { Belted } \\
\text { Kingfisher }\end{array}$ & 50.719 & 100.085 & 469.151 & $3.45 e-01$ \\
\hline & & & $\begin{array}{c}\text { Great Blue } \\
\text { Heron }\end{array}$ & 20.253 & 115.248 & 457.506 & $3.97 e-01$ \\
\hline & & & Barred Owl & 30.132 & 461.644 & 459.680 & \\
\hline & & & Bam Owl & 34.737 & 258.997 & 462.494 & \\
\hline & & & Cooper's Hawk & 35.428 & 457.435 & 457.435 & \\
\hline & & & Red-tailed Hawk & 25.963 & 32.125 & 456.779 & \\
\hline
\end{tabular}


48

Table 12. (continued)

\begin{tabular}{|c|c|c|c|c|c|c|c|}
\hline \multirow{2}{*}{$\begin{array}{l}\text { Contaminant } \\
\text { and Form }\end{array}$} & \multirow{2}{*}{ Test Species } & \multirow{2}{*}{$\begin{array}{c}\text { Test } \\
\text { Species } \\
\text { NOAEL* } \\
\left(\mathbf{m g} / \mathbf{k g} \bullet^{*} \mathbf{d}\right)\end{array}$} & \multirow{2}{*}{$\begin{array}{l}\text { Endpoint } \\
\text { Species }^{b}\end{array}$} & \multirow{2}{*}{$\begin{array}{l}\text { Estimated } \\
\text { Wildlife } \\
\text { NOAELc } \\
\text { (mg/kg • d) }\end{array}$} & \multicolumn{3}{|c|}{ Toxicological Benchmarks } \\
\hline & & & & & $\begin{array}{c}\text { Diet }^{d} \\
(\mathrm{mg} / \mathrm{kg})\end{array}$ & $\begin{array}{l}\text { Watere } \\
\text { (mg/L) }\end{array}$ & $\begin{array}{l}\text { Aquatic } \\
\text { Feeding } \\
\text { Species } \\
\text { (mg/L) }\end{array}$ \\
\hline \multirow[t]{8}{*}{ o-Cresol } & $\min k$ & 216.2 & $\begin{array}{l}\text { Short-tailed } \\
\text { Shrew }\end{array}$ & 864.461 & 1440.768 & 3929.366 & \\
\hline & & & Little Brown Bat & 1086.639 & 3259.916 & 6791.491 & \\
\hline & & & $\begin{array}{l}\text { White-footed } \\
\text { Mouse }\end{array}$ & 761.826 & 4929.463 & 2539.420 & \\
\hline & & & Meadow Vole & 606.060 & 5333.332 & 4444.444 & \\
\hline & & & Cottontail Rabbit & 203.576 & 1030.765 & 2105.959 & \\
\hline & & & Mink & 216.200 & 1578.102 & 2183.838 & $8.49 e+01$ \\
\hline & & & Red Fox & 131.612 & 1316.118 & 1558.560 & \\
\hline & & & Whitetail Deer & 57.105 & 1854.269 & 872.007 & \\
\hline \multirow[t]{8}{*}{ Cyanide } & Rat & 6.87 & $\begin{array}{l}\text { Short-tailed } \\
\text { Shrew }\end{array}$ & 17.897 & 29.828 & 81.350 & \\
\hline & & & Little Brown Bat & 22.497 & 67.490 & 140.605 & \\
\hline & & & $\begin{array}{c}\text { White-footed } \\
\text { Mouse }\end{array}$ & 15.772 & 102.055 & 52.574 & \\
\hline & & & Meadow Vole & 12.547 & 110.416 & 92.014 & \\
\hline & & & Cottontail Rabbit & 4.215 & 21.340 & 43.599 & \\
\hline & & & Mink & 4.476 & 32.672 & 45.212 & $4.52 e+01$ \\
\hline & & & Red Fox & 2.725 & 27.248 & 32.267 & \\
\hline & & & Whitetail Deer & 1.182 & 38.389 & 18.053 & \\
\hline
\end{tabular}


49

Table 12. (continued)

\begin{tabular}{|c|c|c|c|c|c|c|c|}
\hline \multirow{2}{*}{$\begin{array}{l}\text { Contaminant } \\
\text { and Form }\end{array}$} & \multirow{2}{*}{ Test Species } & \multirow{2}{*}{$\begin{array}{c}\text { Test } \\
\text { Species } \\
\text { NOAEL } \\
\left(\mathrm{mg}^{\prime} / \mathrm{kg}^{\bullet} \mathrm{d}\right)\end{array}$} & \multirow{2}{*}{$\begin{array}{l}\text { Endpoint } \\
\text { Species }^{b}\end{array}$} & \multirow{2}{*}{$\begin{array}{c}\text { Estimated } \\
\text { Wildlife } \\
\text { NOAEL } \\
(\mathrm{mg} / \mathrm{kg} \cdot \mathrm{d})\end{array}$} & \multicolumn{3}{|c|}{ Toxicological Benchmarks } \\
\hline & & & & & $\begin{array}{c}\text { Diet }^{d} \\
(\mathbf{m g} / \mathbf{k g})\end{array}$ & $\begin{array}{r}\text { Watere } \\
\text {. (mg/L) }\end{array}$ & $\begin{array}{l}\text { Aquatic } \\
\text { Feeding } \\
\text { Species } \\
\text { (mg/L) }\end{array}$ \\
\hline \multirow[t]{8}{*}{ DDT (and metabolites) } & Rat & 0.8 & $\begin{array}{l}\text { Short-tailed } \\
\text { Shrew }\end{array}$ & 2.262 & 3.770 & 10.283 & \\
\hline & & & Little Brown Bat & 2.844 & 8.531 & 17.772 & \\
\hline & & & $\begin{array}{l}\text { White-footed } \\
\text { Mouse }\end{array}$ & 1.994 & 12.900 & 6.645 & \\
\hline & & & Meadow Vole & 1.586 & 13.956 & 11.630 & \\
\hline & & & Cottontail Rabbit & 0.533 & 2.697 & 5.511 & \\
\hline & & & Mink & 0.566 & 4.130 & 5.715 & $2.64 e-06$ \\
\hline & & & Red Fox & 0.344 & 3.444 & 4.079 & \\
\hline & & & Whitetail Deer & 0.149 & 4.853 & 2.282 & \\
\hline \multirow[t]{9}{*}{ DDT (and metabolites) } & $\begin{array}{l}\text { Brown } \\
\text { Pelican }\end{array}$ & 0.00028 & American Robin & 0.00099 & 0.00082 & 0.00719 & \\
\hline & & & $\begin{array}{l}\text { American } \\
\text { Woodcock }\end{array}$ & 0.00072 & 0.00095 & 0.00713 & \\
\hline & & & Wild Turkey & 0.00024 & 0.008 & 0.00733 & \\
\hline & & & $\begin{array}{c}\text { Belled } \\
\text { Kingfisher }\end{array}$ & 0.0008 & 0.00158 & 0.0074 & $1.01 \mathrm{e}-09$ \\
\hline & & & $\begin{array}{c}\text { Great Blue } \\
\text { Heron }\end{array}$ & 0.00032 & 0.00182 & 0.00723 & $1.16 \mathrm{e}-09$ \\
\hline & & & Barred Owl & 0.00047 & 0.0072 & 0.00717 & \\
\hline & & & Barn Owl & 0.00054 & 0.00403 & 0.00719 & \\
\hline & & & Cooper's Hawk & 0.00056 & 0.00723 & 0.00723 & \\
\hline & & & Red-tailed Hawk & 0.00041 & 0.00051 & 0.00721 & \\
\hline
\end{tabular}


50

Table 12. (continued)

\begin{tabular}{|c|c|c|c|c|c|c|c|}
\hline \multirow[b]{2}{*}{$\begin{array}{l}\text { Contaminant } \\
\text { and Form }\end{array}$} & \multirow{2}{*}{ Test Species } & \multirow{2}{*}{$\begin{array}{c}\text { Test } \\
\text { Species } \\
\text { NOAEL" } \\
(\mathbf{m g} / \mathbf{k g} \cdot \mathbf{d})\end{array}$} & \multirow{2}{*}{$\begin{array}{l}\text { Endpoint } \\
\text { Species }^{b}\end{array}$} & \multirow{2}{*}{$\begin{array}{c}\text { Estimated } \\
\text { Wildlife } \\
\text { NOAEL' } \\
\left(\mathrm{mg} / \mathrm{kg}^{*} \mathrm{~d}\right)\end{array}$} & \multicolumn{3}{|c|}{ Toxicological Benchmarks } \\
\hline & & & & & $\begin{array}{c}\text { Diet }^{d} \\
(\mathrm{mg} / \mathbf{k g})\end{array}$ & $\begin{array}{c}\text { Watere } \\
\text { (mg/L) }\end{array}$ & $\begin{array}{l}\text { Aquatic } \\
\text { Feeding } \\
\text { Species' } \\
\text { (mg/L) }\end{array}$ \\
\hline \multirow[t]{8}{*}{ 1,2-Dichloroethane } & mouse & 50 & $\begin{array}{c}\text { Short-tailed } \\
\text { Shrew }\end{array}$ & 66.131 & 110.218 & 300.593 & \\
\hline & & & Little Brown Bat & 83.127 & 249.381 & 519.544 & \\
\hline & & & $\begin{array}{l}\text { White-footed } \\
\text { Mouse }\end{array}$ & 58.279 & 377.099 & 194.263 & \\
\hline & & & Meadow Vole & 46.363 & 407.994 & 339.995 & \\
\hline & & & Cottontail Rabbit & 15.574 & 78.853 & 161.105 & \\
\hline & & & Mink & 16.539 & 120.723 & 167.061 & $1.41 \mathrm{e}+01$ \\
\hline & & & Red Fox & 10.068 & 100.680 & 119.226 & \\
\hline & & & Whitetail Deer & 4.369 & 141.851 & 66.708 & \\
\hline \multirow[t]{9}{*}{ 1,2-Dichloroethane } & chicken & 17.2 & American Robin & 46.811 & 38.757 & 340.041 & \\
\hline & & & $\begin{array}{l}\text { American } \\
\text { Woodcock }\end{array}$ & 34.276 & 45.244 & 339.331 & \\
\hline & & & Wild Turkey & 11.245 & 374.834 & 343.269 & \\
\hline & & & $\begin{array}{c}\text { Belted } \\
\text { Kingfisher }\end{array}$ & 37.731 & 74.456 & 349.015 & $9.24 e+\infty$ \\
\hline & & & $\begin{array}{c}\text { Great Blue } \\
\text { Heron }\end{array}$ & 15.067 & 85.737 & 340.353 & $1.06 \mathrm{e}+01$ \\
\hline & & & Barred Owl & 22.416 & 343.431 & 341.970 & \\
\hline & & & Barn Owl & 25.842 & 192.675 & 344.063 & \\
\hline & & & Cooper's Hawk & 26.356 & 340.299 & 340.299 & \\
\hline & & & Red-tailed Hawk & 19.314 & 23.899 & 339.813 & \\
\hline
\end{tabular}


51

Table 12. (continued)

\begin{tabular}{|c|c|c|c|c|c|c|c|}
\hline \multirow{2}{*}{$\begin{array}{l}\text { Contaminant } \\
\text { and Form }\end{array}$} & \multirow{2}{*}{ Test Species } & \multirow{2}{*}{$\begin{array}{c}\text { Test } \\
\text { Species } \\
\text { NOAEL" } \\
\left(m g / k g g^{\circ} d\right)\end{array}$} & \multirow{2}{*}{$\begin{array}{l}\text { Endpoint } \\
\text { Species }\end{array}$} & \multirow{2}{*}{$\begin{array}{c}\text { Estimated } \\
\text { Wildlife } \\
\text { NOAELc } \\
\left(\mathbf{m g} / \mathrm{kg}^{\bullet} \mathrm{d}\right)\end{array}$} & \multicolumn{3}{|c|}{ Toxicological Benchmarks } \\
\hline & & & & & $\begin{array}{c}\text { Diet }^{d} \\
(\mathrm{mg} / \mathrm{kg})\end{array}$ & $\begin{array}{l}\text { Water } \\
\text {. (mg/L) }\end{array}$ & $\begin{array}{l}\text { Aquatic } \\
\text { Feeding } \\
\text { Species } \\
\text { (mg/L) }\end{array}$ \\
\hline \multirow[t]{6}{*}{ 1,1-Dichloroethylene } & Rat & 30 & $\begin{array}{l}\text { Short-tailed } \\
\text { Shrew }\end{array}$ & 84.831 & 141.385 & 385.594 & \\
\hline & & & Litule Brown Bat & 106.634 & 319.901 & 666.459 & \\
\hline & & & $\begin{array}{c}\text { White-footed } \\
\text { Mouse }\end{array}$ & 74.759 & 483.735 & 249.197 & \\
\hline & & & Meadow Vole & 59.474 & 523.367 & 436.139 & \\
\hline & & & Cottontail Rabbit & 19.977 & 101.151 & 206.662 & \\
\hline & & & Whitetail Deer & 5.604 & 181.969 & 85.575 & \\
\hline \multirow[t]{2}{*}{ 1,1-Dichloroethylene } & beagle dog & 2.5 & Mink & 5.345 & 39.014 & 53.989 & $1.55 e+\infty$ \\
\hline & & & Red Fox & 3.254 & 32.537 & 38.531 & \\
\hline \multirow[t]{8}{*}{ 1,2-Dichloroethylene } & mouse & 45.2 & $\begin{array}{c}\text { Short-tailed } \\
\text { Shrew }\end{array}$ & 56.817 & 94.695 & 258.258 & \\
\hline & & & Litule Brown Bat & 71.420 & 214.259 & 446.373 & \\
\hline & & & $\begin{array}{c}\text { White-footed } \\
\text { Mouse }\end{array}$ & 50.071 & 323.990 & 166.904 & \\
\hline & & & Meadow Vole & 39.833 & 350.534 & 292.112 & \\
\hline & & & Cottontail Rabbit & 13.380 & 67.747 & 138.415 & \\
\hline & & & Mink & 14.210 & 103.722 & 143.535 & $6.49 e+00$ \\
\hline & & & Red Fox & 8.650 & 86.504 & 102.439 & \\
\hline & & & Whitetail Deer & 3.753 & 121.878 & 57.316 & \\
\hline
\end{tabular}


52

Table 12. (continued)

\begin{tabular}{|c|c|c|c|c|c|c|c|}
\hline \multirow{2}{*}{$\begin{array}{l}\text { Contaminant } \\
\text { and Form }\end{array}$} & \multirow{2}{*}{ Test Species } & \multirow{2}{*}{$\begin{array}{c}\text { Test } \\
\text { Species } \\
\text { NOAEL“ } \\
\left(\mathbf{m g} / \mathbf{k g} \bullet^{\circ} \mathbf{d}\right)\end{array}$} & \multirow{2}{*}{$\begin{array}{l}\text { Endpoint } \\
\text { Species }^{b}\end{array}$} & \multirow{2}{*}{$\begin{array}{c}\text { Estimated } \\
\text { Wildlife } \\
\text { NOAEL } \\
\text { (mg/kg }{ }^{c} \text { d) }\end{array}$} & \multicolumn{3}{|c|}{ Toxicological Benchmarks } \\
\hline & & & & & $\begin{array}{c}\text { Diet }^{d} \\
\text { (mg/kg) }\end{array}$ & $\begin{array}{l}\text { Water } \\
\text { (mg/L) }\end{array}$ & $\begin{array}{c}\text { Aquatic } \\
\text { Feeding } \\
\text { Species } \\
\text { (mg/L) }\end{array}$ \\
\hline \multirow[t]{8}{*}{ Dieldrin } & Rat & 0.02 & $\begin{array}{l}\text { Short-tailed } \\
\text { Shrew }\end{array}$ & 0.057 & 0.094 & 0.257 & \\
\hline & & & Little Brown Bat & 0.071 & 0.213 & 0.444 & \\
\hline & & & $\begin{array}{c}\text { White-footed } \\
\text { Mouse }\end{array}$ & 0.050 & 0.322 & 0.166 & \\
\hline & & & Meadow Vole & 0.040 & 0.349 & 0.291 & \\
\hline & & & Cottontail Rabbit & 0.013 & 0.067 & 0.138 & \\
\hline & & & Mink & 0.014 & 0.103 & 0.143 & $4.61 e-05$ \\
\hline & & & Red Fox & 0.009 & 0.086 & 0.102 & \\
\hline & & & Whitetail Deer & 0.004 & 0.121 & 0.057 & \\
\hline \multirow[t]{9}{*}{ Dieldrin } & Barn Owl & 0.077 & American Robin & 0.139 & 0.115 & 1.013 & \\
\hline & & & $\begin{array}{l}\text { American } \\
\text { Woodcock }\end{array}$ & 0.102 & 0.135 & 1.011 & \\
\hline & & & Wild Turkey & 0.034 & 1.117 & 1.023 & \\
\hline & & & $\begin{array}{c}\text { Belted } \\
\text { Kingfisher }\end{array}$ & 0.112 & 0.222 & 1.040 & $9.92 \mathrm{e}-05$ \\
\hline & & & $\begin{array}{l}\text { Great Blue } \\
\text { Heron }\end{array}$ & 0.045 & 0.255 & 1.014 & $1.14 \mathrm{e}-04$ \\
\hline & & & Barred Owl & 0.067 & 1.023 & 1.019 & \\
\hline & & & Barn Owl & 0.077 & 0.574 & 1.025 & \\
\hline & & & Cooper's Hawk & 0.079 & 1.014 & 1.014 & \\
\hline & & & Red-tailed Hawk & 0.058 & 0.071 & 1.013 & \\
\hline
\end{tabular}


53

Table 12. (continued)

\begin{tabular}{|c|c|c|c|c|c|c|c|}
\hline \multirow{2}{*}{$\begin{array}{l}\text { Contaminant } \\
\text { and Form }\end{array}$} & \multirow{2}{*}{ Test Species } & \multirow{2}{*}{$\begin{array}{c}\text { Test } \\
\text { Species } \\
\text { NOAEL" } \\
\left(\mathbf{m g} / \mathbf{k g} \text { • d) }^{*}\right.\end{array}$} & \multirow{2}{*}{$\begin{array}{l}\text { Endpoint } \\
\text { Species }^{b}\end{array}$} & \multirow{2}{*}{$\begin{array}{l}\text { Estimated } \\
\text { Wildlife } \\
\text { NOAELc } \\
\text { (mg/kg • d) }\end{array}$} & \multicolumn{3}{|c|}{ Toxicological Benchmarks } \\
\hline & & & & & $\begin{array}{c}\text { Diet }^{d} \\
(\mathrm{mg} / \mathrm{kg})\end{array}$ & $\begin{array}{c}\text { Water } \\
\text { (mg/L) }\end{array}$ & $\begin{array}{l}\text { Aquatic } \\
\text { Feeding } \\
\text { Species } \\
\text { (mg/L) }\end{array}$ \\
\hline \multirow[t]{8}{*}{ Diethylphthalate } & mouse & 4583 & $\begin{array}{l}\text { Short-tailed } \\
\text { Shrew }\end{array}$ & 5760.877 & 9601.461 & 26185.804 & \\
\hline & & & Little Brown Bat & 7241.507 & 21724.520 & 45259.417 & \\
\hline & & & $\begin{array}{c}\text { White-footed } \\
\text { Mouse }\end{array}$ & 5076.910 & 32850.594 & 16923.033 & \\
\hline & & & Meadow Vole & 4038.860 & 35541.972 & 29618.310 & \\
\hline & & & Cottontail Rabbit & 1356.660 & 6869.163 & 14034.410 & \\
\hline & & & Mink & 1440.804 & 10516.814 & 14553.571 & $2.33 e+02$ \\
\hline & & & Red Fox & 877.095 & 8770.945 & 10386.646 & \\
\hline & & & Whitetail Deer & 380.572 & 12357.664 & 5811.442 & \\
\hline \multirow[t]{8}{*}{ Di-n-butyl phthalate } & Mouse & 550 & $\begin{array}{l}\text { Short-tailed } \\
\text { Shrew }\end{array}$ & 691.356 & 1152.259 & 3142.525 & \\
\hline & & & Little Brown Bat & 869.044 & 2607.132 & 5431.525 & \\
\hline & & & $\begin{array}{l}\text { White-footed } \\
\text { Mouse }\end{array}$ & 609.274 & 3942.358 & 2030.912 & \\
\hline & & & Meadow Vole & 484.699 & 4265.347 & 3554.456 & \\
\hline & & & Cottontail Rabbit & 162.811 & 824.359 & 1684.252 & \\
\hline & & & Mink & 172.909 & 1262.109 & 1746.556 & $1.41 e+\infty$ \\
\hline & & & Red Fox & 105.259 & 1052.590 & 1246.488 & \\
\hline & & & Whitetail Deer & 45.672 & 1483.028 & 697.424 & \\
\hline
\end{tabular}


54

Table 12. (continued)

\begin{tabular}{|c|c|c|c|c|c|c|c|}
\hline \multirow{2}{*}{$\begin{array}{l}\text { Contaminant } \\
\text { and Form }\end{array}$} & \multirow{2}{*}{ Test Species } & \multirow{2}{*}{$\begin{array}{c}\text { Test } \\
\text { Species } \\
\text { NOAEL" } \\
(\mathbf{m g} / \mathbf{k g} \cdot \mathrm{d})\end{array}$} & \multirow{2}{*}{$\begin{array}{l}\text { Endpoint } \\
\text { Species }^{b}\end{array}$} & \multirow{2}{*}{$\begin{array}{c}\text { Estimated } \\
\text { Wildlife } \\
\text { NOAELc } \\
(\mathrm{mg} / \mathrm{kg} \cdot \mathrm{d})\end{array}$} & \multicolumn{3}{|c|}{ Toxicological Benchmarks } \\
\hline & & & & & $\begin{array}{c}\text { Diet }^{d} \\
(\mathrm{mg} / \mathrm{kg})\end{array}$ & $\begin{array}{l}\text { Watere } \\
\text { (mg/L) }\end{array}$ & $\begin{array}{l}\text { Aquatic } \\
\text { Feeding } \\
\text { Species }^{f} \\
\text { (mg/L) }\end{array}$ \\
\hline \multirow[t]{9}{*}{ Di-n-butyl phthalate } & Ring dove & 0.111 & American Robin & 0.140 & 0.116 & 1.016 & \\
\hline & & & $\begin{array}{l}\text { American } \\
\text { Woodcock }\end{array}$ & 0.102 & 0.135 & 1.014 & \\
\hline & & & Wild Turkey & 0.034 & 1.120 & 1.025 & \\
\hline & & & $\begin{array}{c}\text { Belted } \\
\text { Kingfisher }\end{array}$ & 0.113 & 0.222 & 1.043 & $2.49 e-04$ \\
\hline & & & $\begin{array}{l}\text { Great Blue } \\
\text { Heron }\end{array}$ & 0.045 & 0.256 & 1.017 & $2.87 e-04$ \\
\hline & & & Barred Owl & 0.067 & 1.026 & 1.022 & \\
\hline & & & Barn Owl & 0.077 & 0.576 & 1.028 & \\
\hline & & & Cooper's Hawk & 0.079 & 1.017 & 1.017 & \\
\hline & & & Red-tailed Hawk & 0.058 & 0.071 & 1.015 & \\
\hline \multirow[t]{8}{*}{ Di-n-hexyl phthalate } & mouse & 55 & $\begin{array}{l}\text { Short-tailed } \\
\text { Shrew }\end{array}$ & 69.136 & 115.226 & 314.253 & \\
\hline & & & Little Brown Bat & 86.904 & 260.713 & 543.153 & \\
\hline & & & $\begin{array}{l}\text { White-footed } \\
\text { Mouse }\end{array}$ & 60.927 & 394.236 & 203.091 & \\
\hline & & & Meadow Vole & 48.470 & 426.535 & 355.446 & \\
\hline & & & Cottontail Rabbit & 16.281 & 82.436 & 168.425 & \\
\hline & & & Mink & 17.291 & 126.211 & 174.656 & \\
\hline & & & Red Fox & 10.526 & 105.259 & 124.649 & \\
\hline & & & Whitetail Deer & 4.567 & 148.303 & 69.742 & \\
\hline
\end{tabular}


55

Table 12. (continued)

\begin{tabular}{|c|c|c|c|c|c|c|c|}
\hline \multirow{2}{*}{$\begin{array}{l}\text { Contaminant } \\
\text { and Form }\end{array}$} & \multirow{2}{*}{ Test Species } & \multirow{2}{*}{$\begin{array}{c}\text { Test } \\
\text { Species } \\
\text { NOAEL } \\
(\mathbf{m g} / \mathrm{kg} \cdot \mathrm{d})\end{array}$} & \multirow{2}{*}{$\begin{array}{l}\text { Endpoint } \\
\text { Species }^{b}\end{array}$} & \multirow{2}{*}{$\begin{array}{l}\text { Estimated } \\
\text { Wildlife } \\
\text { NOAEL } \\
(\mathrm{mg} / \mathrm{kg} \bullet \mathrm{d})\end{array}$} & \multicolumn{3}{|c|}{ Toxicological Benchmarks } \\
\hline & & & & & $\begin{array}{c}\text { Diet }^{d} \\
(\mathrm{mg} / \mathrm{kg})\end{array}$ & $\begin{array}{r}\text { Watere } \\
\text { - (mg/L) }\end{array}$ & $\begin{array}{l}\text { Aquatic } \\
\text { Feeding } \\
\text { Species' }^{\text {(mg/L) }}\end{array}$ \\
\hline \multirow[t]{8}{*}{ 1,4-Dioxane } & Rat & 0.5 & $\begin{array}{l}\text { Short-tailed } \\
\text { Shrew }\end{array}$ & 1.414 & 2.356 & 6.427 & \\
\hline & & & Little Brown Bat & 1.777 & 5.332 & 11.108 & \\
\hline & & & $\begin{array}{c}\text { White-footed } \\
\text { Mouse }\end{array}$ & 1.246 & 8.062 & 4.153 & \\
\hline & & & Meadow Vole & 0.991 & 8.723 & 7.269 & \\
\hline & & & Cottontail Rabbit & 0.333 & 1.686 & 3.444 & \\
\hline & & & Mink & 0.354 & 2.581 & 3.572 & $2.37 e+\infty 0$ \\
\hline & & & Red Fox & 0.215 & 2.153 & 2.549 & \\
\hline & & & Whitetail Deer & 0.093 & 3.033 & 1.426 & \\
\hline \multirow[t]{8}{*}{ Endosulfan } & Rat & 0.15 & $\begin{array}{c}\text { Short-tailed } \\
\text { Shrew }\end{array}$ & 0.424 & 0.707 & 1.928 & \\
\hline & & & Little Brown Bat & 0.533 & 1.600 & 3.332 & \\
\hline & & & $\begin{array}{l}\text { White-footed } \\
\text { Mouse }\end{array}$ & 0.374 & 2.419 & 1.246 & \\
\hline & & & Meadow Vole & 0.297 & 2.617 & 2.181 & \\
\hline & & & Cottontail Rabbit & 0.100 & 0.506 & 1.033 & \\
\hline & & & Mink & 0.106 & 0.774 & 1.072 & \\
\hline & & & Red Fox & 0.065 & 0.646 & 0.765 & \\
\hline & & & Whitetail Deer & 0.028 & 0.910 & 0.428 & \\
\hline
\end{tabular}


56

Table 12. (continued)

\begin{tabular}{|c|c|c|c|c|c|c|c|}
\hline \multirow{2}{*}{$\begin{array}{l}\text { Contaminant } \\
\text { and Form }\end{array}$} & \multirow{2}{*}{ Test Species } & \multirow{2}{*}{$\begin{array}{c}\text { Test } \\
\text { Species } \\
\text { NOAEL } \\
\left(\mathbf{m g} / \mathbf{k g} \bullet^{\circ} \mathbf{d}\right)\end{array}$} & \multirow{2}{*}{$\begin{array}{l}\text { Endpoint } \\
\text { Species }^{b}\end{array}$} & \multirow{2}{*}{$\begin{array}{c}\text { Estimated } \\
\text { Wildlife } \\
\text { NOAEL } \\
\left(\mathrm{mg} / \mathrm{kg}^{\bullet} \mathrm{d}\right)\end{array}$} & \multicolumn{3}{|c|}{ Toxicological Benchmarks } \\
\hline & & & & & $\begin{array}{c}\text { Diet }^{d} \\
\text { (mg/kg) }\end{array}$ & $\begin{array}{l}\text { Water } \\
\text { (mg/L) }\end{array}$ & $\begin{array}{l}\text { Aquatic } \\
\text { Feeding } \\
\text { Species } \\
\text { (mg/L) }\end{array}$ \\
\hline \multirow[t]{9}{*}{ Endosulfan } & $\begin{array}{l}\text { Gray } \\
\text { Partridge }\end{array}$ & 10 & American Robin & 17.224 & 14.261 & 125.119 & \\
\hline & & & $\begin{array}{l}\text { American } \\
\text { Woodcock }\end{array}$ & 12.612 & 16.648 & 124.858 & \\
\hline & & & Wild Turkey & 4.138 & 137.920 & 126.306 & \\
\hline & & & $\begin{array}{c}\text { Belted } \\
\text { Kingfisher }\end{array}$ & 13.883 & 27.396 & 128.421 & \\
\hline & & & $\begin{array}{l}\text { Great Blue } \\
\text { Heron }\end{array}$ & 5.544 & 31.547 & 125.233 & \\
\hline & & & Barred Owl & 8.248 & 126.367 & 125.829 & \\
\hline & & & Bam Owl & 9.509 & 70.895 & 126.599 & \\
\hline & & & Cooper's Hawk & 9.698 & 125.213 & 125.213 & \\
\hline & & & Red-tailed Hawk & 7.107 & 8.794 & 125.035 & \\
\hline \multirow[t]{8}{*}{ Endrin } & Mouse & 0.092 & $\begin{array}{c}\text { Short-tailed } \\
\text { Shrew }\end{array}$ & 0.116 & 0.193 & 0.526 & \\
\hline & & & Little Brown Bat & 0.145 & 0.436 & 0.909 & \\
\hline & & & $\begin{array}{c}\text { White-footed } \\
\text { Mouse }\end{array}$ & 0.102 & 0.659 & 0.340 & \\
\hline & & & Meadow Vole & 0.081 & 0.714 & 0.595 & \\
\hline & & & Cottontail Rabbit & 0.027 & 0.138 & 0.282 & \\
\hline & & & Mink & 0.029 & 0.211 & 0.292 & $9.44 e-05$ \\
\hline & & & Red Fox & 0.018 & 0.176 & 0.209 & \\
\hline & & & Whitetail Deer & 0.008 & 0.248 & 0.117 & \\
\hline
\end{tabular}


Table 12. (continued)

\begin{tabular}{|c|c|c|c|c|c|c|c|}
\hline \multirow{2}{*}{$\begin{array}{l}\text { Contaminant } \\
\text { and Form }\end{array}$} & \multirow{2}{*}{ Test Species } & \multirow{2}{*}{$\begin{array}{c}\text { Test } \\
\text { Species } \\
\text { NOAEL" } \\
\left(\mathrm{mg} / \mathrm{kg} \bullet^{\circ} \mathrm{d}\right)\end{array}$} & \multirow{2}{*}{$\begin{array}{l}\text { Endpoint } \\
\text { Species }^{b}\end{array}$} & \multirow{2}{*}{$\begin{array}{c}\text { Estimated } \\
\text { Wildlife } \\
\text { NOAEL } \\
(\mathrm{mg} / \mathrm{kg} \cdot \mathrm{d})\end{array}$} & \multicolumn{3}{|c|}{ Toxicological Benchmarks } \\
\hline & & & & & $\begin{array}{c}\text { Diet }^{d} \\
(\mathrm{mg} / \mathrm{kg})\end{array}$ & $\begin{array}{c}\text { Watere } \\
\text { (mg/L) }\end{array}$ & $\begin{array}{l}\text { Aquatic } \\
\text { Feeding } \\
\text { Species } \\
\text { (mg/L) }\end{array}$ \\
\hline \multirow[t]{9}{*}{ Endrin } & Mallard Duck & 0.3 & American Robin & 0.732 & 0.606 & 5.319 & \\
\hline & & & $\begin{array}{l}\text { American } \\
\text { Woodcock }\end{array}$ & 0.536 & 0.708 & 5.307 & \\
\hline & & & Wild Turkey & 0.176 & 5.863 & 5.369 & \\
\hline & & & $\begin{array}{c}\text { Belted } \\
\text { Kingfisher }\end{array}$ & 0.590 & 1.165 & 5.459 & $5.21 \mathrm{e}-04$ \\
\hline & & & $\begin{array}{c}\text { Great Blue } \\
\text { Heron }\end{array}$ & 0.236 & 1.341 & 5.324 & $6.00 \mathrm{e}-04$ \\
\hline & & & Barred Owl & 0.351 & 5.372 & 5.349 & \\
\hline & & & Barn Owl & 0.404 & 3.014 & 5.382 & \\
\hline & & & Cooper's Hawk & 0.412 & 5.323 & 5.323 & \\
\hline & & & Red-tailed Hawk & 0.302 & 0.374 & 5.315 & \\
\hline \multirow[t]{8}{*}{ Ethanol } & Rat & 31.9 & $\begin{array}{c}\text { Short-tailed } \\
\text { Shrew }\end{array}$ & 90.203 & 150.339 & 410.015 & \\
\hline & & & Little Brown Bat & 113.387 & 340.161 & 708.669 & \\
\hline & & & $\begin{array}{l}\text { White-footed } \\
\text { Mouse }\end{array}$ & 79.494 & 514.372 & 264.979 & \\
\hline & & & Meadow Vole & 63.240 & 556.513 & 463.761 & \\
\hline & & & Cottontail Rabbit & 21.243 & 107.557 & 219.750 & \\
\hline & & & Mink & 22.560 & 164.669 & 227.876 & $1.55 e+02$ \\
\hline & & & Red Fox & 13.733 & 137.333 & 162.631 & \\
\hline & & & Whitetail Deer & 5.959 & 193.494 & 90.994 & \\
\hline
\end{tabular}


58

Table 12. (continued)

\begin{tabular}{|c|c|c|c|c|c|c|c|}
\hline \multirow{2}{*}{$\begin{array}{l}\text { Contaminant } \\
\text { and Form }\end{array}$} & \multirow{2}{*}{ Test Species } & \multirow{2}{*}{$\begin{array}{c}\text { Test } \\
\text { Species } \\
\text { NOAEL: } \\
\left(\mathrm{mg}_{\mathrm{g} / \mathrm{kg}}^{\circ} \mathrm{d}\right)\end{array}$} & \multirow{2}{*}{$\begin{array}{l}\text { Endpoint } \\
\text { Species }^{\mathrm{b}}\end{array}$} & \multirow{2}{*}{$\begin{array}{c}\text { Estimated } \\
\text { Wildlife } \\
\text { NOAEL } \\
\left(\mathrm{mg} / \mathrm{kg}^{\circ} \mathrm{d}\right)\end{array}$} & \multicolumn{3}{|c|}{ Toxicological Benchmarks } \\
\hline & & & & & $\underset{\text { (mg/kg) }}{\text { Diet }^{d}}$ & $\begin{array}{l}\text { Watere } \\
\text { (mg/L) }\end{array}$ & $\begin{array}{c}\text { Aquatic } \\
\text { Feeding } \\
\text { Speciess } \\
\text { (mg/L) }\end{array}$ \\
\hline \multirow[t]{8}{*}{ Ethyl Acetate } & Rat & 90 & $\begin{array}{l}\text { Short-tailed } \\
\text { Shrew }\end{array}$ & 254.492 & 424.154 & 1156.782 & \\
\hline & & & Little Brown Bat & 319.901 & 959.702 & 1999.378 & \\
\hline & & & $\begin{array}{c}\text { White-footed } \\
\text { Mouse }\end{array}$ & 224.277 & 1451.206 & 747.591 & \\
\hline & & & Meadow Vole & 178.421 & 1570.100 & 1308.417 & \\
\hline & & & Cottontail Rabbit & 59.932 & 303.453 & 619.985 & \\
\hline & & & Mink & 63.648 & 464.584 & 642.909 & \\
\hline & & & Red Fox & 38.746 & 387.459 & 458.833 & \\
\hline & & & Whitetail Deer & 16.812 & 545.907 & 256.724 & \\
\hline Fluoride & $\operatorname{mink}$ & 31.37 & $\begin{array}{l}\text { Short-tailed } \\
\text { Shrew }\end{array}$ & 125.431 & 209.051 & 570.140 & \\
\hline \multirow[t]{7}{*}{$\mathrm{NaF}$} & & & Little Brown Bat & 157.668 & 473.004 & 985.426 & \\
\hline & & & $\begin{array}{l}\text { White-footed } \\
\text { Mouse }\end{array}$ & 110.539 & 715.251 & 368.463 & \\
\hline & & & Meadow Vole & 87.938 & 773.851 & 644.876 & \\
\hline & & & Cottontail Rabbit & 29.538 & 149.561 & 305.569 & \\
\hline & & & Mink & 31.370 & 228.978 & 316.869 & \\
\hline & & & Red Fox & 19.096 & 190.965 & 226.143 & \\
\hline & & & Whitetail Deer & 8.286 & 269.049 & 126.526 & \\
\hline
\end{tabular}


Table 12. (continued)

\begin{tabular}{|c|c|c|c|c|c|c|c|}
\hline \multirow{2}{*}{$\begin{array}{l}\text { Contaminant } \\
\text { and Form }\end{array}$} & \multirow{2}{*}{ Test Species } & \multirow{2}{*}{$\begin{array}{c}\text { Test } \\
\text { Species } \\
\text { NOAEL" } \\
(\mathrm{mg} / \mathrm{kg} \bullet \mathrm{d})\end{array}$} & \multirow{2}{*}{$\begin{array}{l}\text { Endpoint } \\
\text { Species }^{b}\end{array}$} & \multirow{2}{*}{$\begin{array}{c}\text { Estimated } \\
\text { Wildlife } \\
\text { NOAELc } \\
(\mathbf{m g} / \mathbf{k g} \cdot \mathbf{d})\end{array}$} & \multicolumn{3}{|c|}{ Toxicological Benchmarks } \\
\hline & & & & & $\begin{array}{c}\text { Diet }^{d} \\
(\mathrm{mg} / \mathrm{kg})\end{array}$ & $\begin{array}{c}\text { Water } \\
\text { - }(\mathrm{mg} / \mathrm{L})\end{array}$ & $\begin{array}{c}\text { Aquatic } \\
\text { Feeding } \\
\text { Species } \\
\text { (mg/L) }\end{array}$ \\
\hline Fluoride & Sereech Owl & 7.8 & American Robin & 10.342 & 8.562 & 75.123 & \\
\hline \multirow[t]{8}{*}{$\mathrm{NaF}$} & & & $\begin{array}{l}\text { American } \\
\text { Woodcock }\end{array}$ & 7.572 & 9.995 & 74.966 & \\
\hline & & & Wild Turkey & 2.484 & 82.810 & 75.837 & \\
\hline & & & $\begin{array}{c}\text { Belted } \\
\text { Kingfisher }\end{array}$ & 8.336 & 16.449 & 77.105 & \\
\hline & & & $\begin{array}{c}\text { Great Blue } \\
\text { Heron }\end{array}$ & 3.329 & 18.941 & 75.192 & \\
\hline & & & Barred Owl & 4.952 & 75.872 & 75.549 & \\
\hline & & & Bam Owl & 5.709 & 42.566 & 76.011 & \\
\hline & & & Cooper's Hawk & 5.823 & 75.179 & 75.179 & \\
\hline & & & Red-tailed Hawk & 4.267 & 5.280 & 75.072 & \\
\hline \multirow[t]{8}{*}{ Formaldehyde } & beagle dog & 9.4 & $\begin{array}{c}\text { Short-tailed } \\
\text { Shrew }\end{array}$ & 85.339 & 142.232 & 387.905 & \\
\hline & & & Little Brown Bat & 107.272 & 321.817 & 670.452 & \\
\hline & & & $\begin{array}{l}\text { White-footed } \\
\text { Mouse }\end{array}$ & 75.207 & 486.633 & 250.690 & \\
\hline & & & Meadow Vole & 59.830 & 526.503 & 438.752 & \\
\hline & & & Cottontail Rabbit & 20.097 & 101.756 & 207.898 & \\
\hline & & & Mink & 21.343 & 155.789 & 215.587 & $8.61 e+01$ \\
\hline & & & Red Fox & 12.993 & 129.927 & 153.861 & \\
\hline & & & Whitetail Deer & 5.637 & 183.055 & 86.086 & \\
\hline
\end{tabular}


60

Table 12. (continued)

\begin{tabular}{|c|c|c|c|c|c|c|c|}
\hline \multirow{2}{*}{$\begin{array}{l}\text { Contaminant } \\
\text { and Form }\end{array}$} & \multirow{2}{*}{ Test Species } & \multirow{2}{*}{$\begin{array}{c}\text { Test } \\
\text { Species } \\
\text { NOAEL" } \\
\left(\mathbf{m g} / \mathbf{k g}^{\bullet} \mathbf{d}\right)\end{array}$} & \multirow{2}{*}{$\begin{array}{l}\text { Endpoint } \\
\text { Species }^{b}\end{array}$} & \multirow{2}{*}{$\begin{array}{c}\text { Estimated } \\
\text { Wildlife } \\
\text { NOAEL' } \\
(\mathrm{mg} / \mathrm{kg} \bullet \mathrm{d})\end{array}$} & \multicolumn{3}{|c|}{ Toxicological Benchmarks } \\
\hline & & & & & $\begin{array}{c}\text { Diet }^{d} \\
\text { (mg/kg) }\end{array}$ & $\begin{array}{l}\text { Water } \\
\text { (mg/L) }\end{array}$ & $\begin{array}{l}\text { Aquatic } \\
\text { Feeding } \\
\text { Species } \\
\text { (mg/L) }\end{array}$ \\
\hline \multirow[t]{8}{*}{ Heptachlor } & Rat & 0.8 & $\begin{array}{l}\text { Short-tailed } \\
\text { Shrew }\end{array}$ & 2.262 & 3.770 & 10.283 & \\
\hline & & & Litule Brown Bat & 2.844 & 8.531 & 17.772 & \\
\hline & & & $\begin{array}{l}\text { White-footed } \\
\text { Mouse }\end{array}$ & 1.994 & 12.900 & 6.645 & \\
\hline & & & Meadow Vole & 1.586 & 13.956 & 11.630 & \\
\hline & & & Cottontail Rabbit & 0.533 & 2.697 & 5.511 & \\
\hline & & & Mink & 0.566 & 4.130 & 5.715 & $3.62 \mathrm{e}-03$ \\
\hline & & & Red Fox & 0.344 & 3.444 & 4.079 & \\
\hline & & & Whitetail Deer & 0.149 & 4.853 & 2.282 & \\
\hline \multirow[t]{8}{*}{$\begin{array}{l}1,2,3,6,7,8-\text { Hexachloro } \\
\text { Dibenzofuran }\end{array}$} & Rat & 0.00016 & $\begin{array}{l}\text { Short-tailed } \\
\text { Shrew }\end{array}$ & 0.00045 & 0.00075 & 0.00205 & \\
\hline & & & Little Brown Bat & 0.001 & 0.002 & 0.004 & \\
\hline & & & $\begin{array}{l}\text { White-footed } \\
\text { Mouse }\end{array}$ & 0.0004 & 0.00259 & 0.00133 & \\
\hline & & & Meadow Vole & 0.00032 & 0.00282 & 0.00235 & \\
\hline & & & Cottontail Rabbit & 0.00011 & 0.00056 & 0.00114 & \\
\hline & & & Mink & 0.00011 & 0.0008 & 0.00111 & \\
\hline & & & Red Fox & 0.00007 & 0.0007 & 0.00083 & \\
\hline & & & Whitetail Deer & 0.00003 & 0.00097 & 0.00046 & \\
\hline
\end{tabular}


61

Table 12. (continued)

\begin{tabular}{|c|c|c|c|c|c|c|c|}
\hline \multirow{2}{*}{$\begin{array}{l}\text { Contaminant } \\
\text { and Form }\end{array}$} & \multirow{2}{*}{ Test Species } & \multirow{2}{*}{$\begin{array}{c}\text { Test } \\
\text { Species } \\
\text { NOAEL' } \\
\left(\mathrm{mg} / \mathrm{kg}^{*} \mathrm{~d}\right)\end{array}$} & \multirow{2}{*}{$\begin{array}{l}\text { Endpoint } \\
\text { Species }^{b}\end{array}$} & \multirow{2}{*}{$\begin{array}{c}\text { Estimated } \\
\text { Wildlife } \\
\text { NOAELc } \\
\left(\mathrm{mg} / \mathrm{kg}^{\circ} \mathrm{d}\right)\end{array}$} & \multicolumn{3}{|c|}{ Toxicological Benchmarks } \\
\hline & & & & & $\begin{array}{c}\text { Diet }^{d} \\
(\mathbf{m g} / \mathbf{k g})\end{array}$ & $\begin{array}{l}\text { Watere } \\
\text { (mg/L) }\end{array}$ & $\begin{array}{l}\text { Aquatic } \\
\text { Feeding } \\
\text { Species } \\
\text { (mg/L) }\end{array}$ \\
\hline Lead & Rat & 8 & $\begin{array}{c}\text { Short-tailed } \\
\text { Shrew }\end{array}$ & 22.622 & 37.703 & 102.825 & \\
\hline \multirow[t]{7}{*}{ Lead Acetate } & & & Little Brown Bat & 28.436 & 85.307 & 177.723 & \\
\hline & & & $\begin{array}{c}\text { White-footed } \\
\text { Mouse }\end{array}$ & 19.936 & 128.996 & 66.453 & \\
\hline & & & Meadow Vole & 15.860 & 139.564 & 116.304 & \\
\hline & & & Cottontail Rabbit & 5.327 & 26.974 & 55.110 & \\
\hline & & & Mink & 5.658 & 41.296 & 57.147 & $9.03 e-01$ \\
\hline & & & Red Fox & 3.444 & 34.441 & 40.785 & \\
\hline & & & Whitetail Deer & 1.494 & 48.525 & 22.820 & \\
\hline Lead & $\begin{array}{l}\text { American } \\
\text { Kestrel }\end{array}$ & 3.85 & American Robin & 4.576 & 3.789 & 33.243 & \\
\hline \multirow[t]{8}{*}{ Metal } & & & $\begin{array}{l}\text { American } \\
\text { Woodcock }\end{array}$ & 3.351 & 4.423 & 33.174 & \\
\hline & & & Wild Turkey & 1.099 & 36.644 & 33.558 & \\
\hline & & & $\begin{array}{c}\text { Belted } \\
\text { Kingfisher }\end{array}$ & 3.689 & 7.279 & 34.121 & $1.61 \mathrm{e}-01$ \\
\hline & & & $\begin{array}{c}\text { Great Blue } \\
\text { Heron }\end{array}$ & 1.473 & 8.382 & 33.274 & $1.85 \mathrm{e}-01$ \\
\hline & & & Barred Owl & 2.192 & 33.575 & 33.432 & \\
\hline & & & Barn Owl & 2.526 & 18.837 & 33.637 & \\
\hline & & & Cooper's Hawk & 2.577 & 33.269 & 33.269 & \\
\hline & & & Red-tailed Hawk & 1.888 & 2.336 & 33.221 & \\
\hline
\end{tabular}


62

Table 12. (continued)

\begin{tabular}{|c|c|c|c|c|c|c|c|}
\hline \multirow[b]{2}{*}{$\begin{array}{l}\text { Contaminant } \\
\text { and Form }\end{array}$} & \multirow{2}{*}{ Test Species } & \multirow{2}{*}{$\begin{array}{c}\text { Test } \\
\text { Species } \\
\text { NOAEL` } \\
(\mathrm{mg} / \mathrm{kg} \bullet \mathrm{d})\end{array}$} & \multirow[b]{2}{*}{$\begin{array}{l}\text { Endpoint } \\
\text { Species }^{b}\end{array}$} & \multirow{2}{*}{$\begin{array}{c}\text { Estimated } \\
\text { Wildlife } \\
\text { NOAELc } \\
\left(\mathrm{mg} / \mathrm{kg}^{\bullet} \cdot \mathrm{d}\right)\end{array}$} & \multicolumn{3}{|c|}{ Toxicological Benchmarks } \\
\hline & & & & & $\begin{array}{c}\text { Diet }^{d} \\
\text { (mg/kg) }\end{array}$ & $\begin{array}{l}\text { Watere } \\
\text { (mg/L) }\end{array}$ & $\begin{array}{c}\text { Aquatic } \\
\text { Feeding } \\
\text { Species' } \\
\text { (mg/L) }\end{array}$ \\
\hline \multirow[t]{8}{*}{ Lindane (Gamma-BHC) } & Rat & 8 & $\begin{array}{c}\text { Short-tailed } \\
\text { Shrew }\end{array}$ & 22.622 & 37.703 & 102.825 & \\
\hline & & & Little Brown Bat & 28.436 & 85.307 & 177.723 & \\
\hline & & & $\begin{array}{c}\text { White-footed } \\
\text { Mouse }\end{array}$ & 19.936 & 128.996 & 66.453 & \\
\hline & & & Meadow Vole & 15.860 & 139.564 & 116.304 & \\
\hline & & & Cottontail Rabbit & 5.327 & 26.974 & 55.110 & \\
\hline & & & Mink & 5.658 & 41.296 & 57.147 & $1.04 e-01$ \\
\hline & & & Red Fox & 3.444 & 34.441 & 40.785 & \\
\hline & & & Whitetail Deer & 1.494 & 48.525 & 22.820 & \\
\hline \multirow[t]{9}{*}{ Lindane (Gamma-BHC) } & malland duck & 2 & American Robin & 4.661 & 3.859 & 33.859 & \\
\hline & & & $\begin{array}{l}\text { American } \\
\text { Woodcock }\end{array}$ & 3.413 & 4.505 & 33.788 & \\
\hline & & & Wild Turkey & 1.120 & 37.323 & 34.180 & \\
\hline & & & $\begin{array}{c}\text { Belted } \\
\text { Kingfisher }\end{array}$ & 3.757 & 7.414 & 34.752 & $1.87 e-02$ \\
\hline & & & $\begin{array}{c}\text { Great Blue } \\
\text { Heron }\end{array}$ & 1.500 & 8.537 & 33.890 & $2.16 \mathrm{e}-02$ \\
\hline & & & Barred Owl & 2.232 & 34.197 & 34.051 & \\
\hline & & & Barn Owl & 2.573 & 19.185 & 34.260 & \\
\hline & & & Cooper's Hawk & 2.624 & 33.885 & 33.885 & \\
\hline & & & Red-tailed Hawk & 1.923 & 2.380 & 33.836 & \\
\hline
\end{tabular}


Table 12. (continued)

\begin{tabular}{|c|c|c|c|c|c|c|c|}
\hline \multirow{2}{*}{$\begin{array}{l}\text { Contaminant } \\
\text { and Form }\end{array}$} & \multirow{2}{*}{ Test Species } & \multirow{2}{*}{$\begin{array}{c}\text { Test } \\
\text { Species } \\
\text { NOAEL' } \\
(\text { mg/kg・d) }\end{array}$} & \multirow{2}{*}{$\begin{array}{l}\text { Endpoint } \\
\text { Species }^{b}\end{array}$} & \multirow{2}{*}{$\begin{array}{c}\text { Estimated } \\
\text { Wildlife } \\
\text { NOAEL } \\
(\mathrm{mg} / \mathrm{kg} \cdot \mathrm{d})\end{array}$} & \multicolumn{3}{|c|}{ Toxicological Benchmarks } \\
\hline & & & & & $\begin{array}{c}\text { Diet }^{d} \\
(\mathrm{mg} / \mathrm{kg})\end{array}$ & $\begin{array}{r}\text { Watere } \\
\cdot(\mathrm{mg} / \mathrm{L})\end{array}$ & $\begin{array}{l}\text { Aquatic } \\
\text { Feeding } \\
\text { Speciest } \\
\text { (mg/L) }\end{array}$ \\
\hline Lithium & Rat & 9.39 & $\begin{array}{c}\text { Short-tailed } \\
\text { Shrew }\end{array}$ & 26.552 & 44.253 & 120.691 & \\
\hline \multirow[t]{7}{*}{ Lithium Carbonate } & & & Little Brown Bat & 33.376 & 100.129 & 208.602 & \\
\hline & & & $\begin{array}{l}\text { White-footed } \\
\text { Mouse }\end{array}$ & 23.400 & 151.409 & 77.999 & \\
\hline & & & Meadow Vole & 18.615 & 163.814 & 136.512 & \\
\hline & & & Cottontail Rabbit & 6.253 & 31.660 & 64.685 & \\
\hline & & & Mink & 6.641 & 48.472 & 67.077 & \\
\hline & & & Red Fox & 4.042 & 40.425 & 47.872 & \\
\hline & & & Whitetail Deer & 1.754 & 56.956 & 26.785 & \\
\hline Manganese & Rat & 88 & $\begin{array}{l}\text { Short-tailed } \\
\text { Shrew }\end{array}$ & 248.837 & 414.728 & 1131.076 & \\
\hline \multirow[t]{7}{*}{ Manganese Oxide } & & & Little Brown Bat & 312.792 & 938.375 & 1954.948 & \\
\hline & & & $\begin{array}{l}\text { White-footed } \\
\text { Mouse }\end{array}$ & 219.293 & 1418.957 & 730.978 & \\
\hline & & & Meadow Vole & 174.456 & 1535.209 & 1279.341 & \\
\hline & & & Cottontail Rabbit & 58.600 & 296.709 & 606.208 & \\
\hline & & & Mink & 62.234 & 454.260 & 628.622 & \\
\hline & & & Red Fox & 37.885 & 378.849 & 448.637 & \\
\hline & & & Whitetail Deer & 16.438 & 533.776 & 251.019 & \\
\hline
\end{tabular}


64

Table 12. (continued)

\begin{tabular}{|c|c|c|c|c|c|c|c|}
\hline \multirow[b]{2}{*}{$\begin{array}{l}\text { Contaminant } \\
\text { and Form }\end{array}$} & \multirow{2}{*}{ Test Species } & \multirow{2}{*}{$\begin{array}{c}\text { Test } \\
\text { Species } \\
\text { NOAEL" } \\
(\mathbf{m g} / \mathbf{k g} \cdot \mathrm{d})\end{array}$} & \multirow{2}{*}{$\begin{array}{l}\text { Endpoint } \\
\text { Species }\end{array}$} & \multirow{2}{*}{$\begin{array}{c}\text { Estimated } \\
\text { Wildlife } \\
\text { NOAELc } \\
\left(\mathrm{mg} / \mathrm{kg}^{\circ} \mathrm{d}\right)\end{array}$} & \multicolumn{3}{|c|}{ Toxicological Benchmarks } \\
\hline & & & & & $\begin{array}{c}\text { Diet }^{d} \\
\text { (mg/kg) }\end{array}$ & $\begin{array}{l}\text { Water } \\
(\mathrm{mg} / \mathrm{L})\end{array}$ & $\begin{array}{l}\text { Aquatic } \\
\text { Feeding } \\
\text { Species } \\
\text { (mg/L) }\end{array}$ \\
\hline Mercury & Rat & 0.0064 & $\begin{array}{c}\text { Short-tailed } \\
\text { Shrew }\end{array}$ & 0.018 & 0.030 & 0.082 & \\
\hline \multirow[t]{7}{*}{ Mercuric Chloride } & & & Little Brown Bat & 0.023 & 0.068 & 0.142 & \\
\hline & & & $\begin{array}{l}\text { White-footed } \\
\text { Mouse }\end{array}$ & 0.016 & 0.103 & 0.053 & \\
\hline & & & Meadow Vole & 0.013 & 0.112 & 0.093 & \\
\hline & & & Cottontail Rabbit & 0.004 & 0.022 & 0.044 & \\
\hline & & & Mink & 0.005 & 0.033 & 0.046 & \\
\hline & & & Red Fox & 0.003 & 0.028 & 0.033 & \\
\hline & & & Whitetail Deer & 0.001 & 0.039 & 0.018 & \\
\hline Mercury & mouse & 13.2 & $\begin{array}{l}\text { Short-tailed } \\
\text { Shrew }\end{array}$ & 16.593 & 27.654 & 75.421 & \\
\hline \multirow[t]{7}{*}{ Mercuric Sulfide } & & & Little Brown Bat & 20.857 & 62.571 & 130.357 & \\
\hline & & & $\begin{array}{l}\text { White-footed } \\
\text { Mouse }\end{array}$ & 14.623 & 94.617 & 48.742 & \\
\hline & & & Meadow Vole & 11.633 & 102.368 & 85.307 & \\
\hline & & & Cottontail Rabbit & 3.907 & 19.785 & 40.422 & \\
\hline & & & Mink & 4.150 & 30.291 & 41.917 & \\
\hline & & & Red Fox & 2.526 & 25.262 & 29.916 & \\
\hline & & & Whitetail Deer & 1.096 & 35.593 & 16.738 & \\
\hline
\end{tabular}


65

Table 12. (continued)

\begin{tabular}{|c|c|c|c|c|c|c|c|}
\hline \multirow{2}{*}{$\begin{array}{l}\text { Contaminant } \\
\text { and Form }\end{array}$} & \multirow{2}{*}{ Test Species } & \multirow{2}{*}{$\begin{array}{c}\text { Test } \\
\text { Species } \\
\text { NOAEL" }^{\mathrm{a}} \\
\left(\mathrm{mg} / \mathrm{kg}^{\bullet} \mathrm{d}\right)\end{array}$} & \multirow{2}{*}{$\begin{array}{l}\text { Endpoint } \\
\text { Species }^{b}\end{array}$} & \multirow{2}{*}{$\begin{array}{c}\text { Estimated } \\
\text { Wildlife } \\
\text { NOAELc } \\
\left(\mathrm{mg} / \mathrm{kg}^{\bullet} \mathrm{d}\right)\end{array}$} & \multicolumn{3}{|c|}{ Toxicological Benchmarks } \\
\hline & & & & & $\begin{array}{c}\text { Diet }^{d} \\
\text { (mg/kg) }\end{array}$ & $\begin{array}{r}\text { Water } \\
\text { - (mg/L) }\end{array}$ & $\begin{array}{l}\text { Aquatic } \\
\text { Feeding } \\
\text { Species } \\
\text { (mg/L) }\end{array}$ \\
\hline Mercury & Rat & 0.032 & $\begin{array}{l}\text { Short-tailed } \\
\text { Shrew }\end{array}$ & 0.090 & 0.151 & 0.411 & \\
\hline \multirow{5}{*}{$\begin{array}{c}\text { Methyl Mercury } \\
\text { Chloride }\end{array}$} & & & Little Brown Bat & 0.114 & 0.341 & 0.711 & \\
\hline & & & $\begin{array}{c}\text { White-footed } \\
\text { Mouse }\end{array}$ & 0.080 & 0.516 & 0.266 & \\
\hline & & & Meadow Vole & 0.063 & 0.558 & 0.465 & \\
\hline & & & Cottontail Rabbit & 0.021 & 0.108 & 0.220 & \\
\hline & & & Whitetail Deer & 0.006 & 0.194 & 0.091 & \\
\hline Mercury & $\operatorname{mink}$ & 0.015 & Mink & 0.015 & 0.109 & 0.152 & $1.82 e-06$ \\
\hline $\begin{array}{l}\text { Methyl Mercury } \\
\text { Chloride }\end{array}$ & & & Red Fox & 0.009 & 0.091 & 0.108 & \\
\hline Mercury & mallard duck & 0.0064 & American Robin & 0.015 & 0.012 & 0.108 & \\
\hline \multirow[t]{8}{*}{$\begin{array}{l}\text { Methyl Mercury } \\
\text { Dicyandiamide }\end{array}$} & & & $\begin{array}{l}\text { American } \\
\text { Woodcock }\end{array}$ & 0.011 & 0.014 & 0.108 & \\
\hline & & & Wild Turkey & 0.004 & 0.119 & 0.109 & \\
\hline & & & $\begin{array}{c}\text { Belted } \\
\text { Kingfisher }\end{array}$ & 0.012 & 0.024 & 0.111 & $3.95 e-07$ \\
\hline & & & $\begin{array}{l}\text { Great Blue } \\
\text { Heron }\end{array}$ & 0.005 & 0.027 & 0.108 & $4.55 e-07$ \\
\hline & & & Barred Owl & 0.007 & 0.109 & 0.109 & \\
\hline & & & Barn Owl & 0.008 & 0.061 & 0.110 & \\
\hline & & & Cooper's Hawk & 0.008 & 0.108 & 0.108 & \\
\hline & & & Red-tailed Hawk & 0.006 & 0.008 & 0.108 & \\
\hline
\end{tabular}


66

Table 12. (continued)

\begin{tabular}{|c|c|c|c|c|c|c|c|}
\hline \multirow{2}{*}{$\begin{array}{l}\text { Contaminant } \\
\text { and Form }\end{array}$} & \multirow{2}{*}{ Test Species } & \multirow{2}{*}{$\begin{array}{c}\text { Test } \\
\text { Species } \\
\text { NOAEL } \\
(m g / k g \cdot d)\end{array}$} & \multirow{2}{*}{$\begin{array}{l}\text { Endpoint } \\
\text { Species }^{b}\end{array}$} & \multirow{2}{*}{$\begin{array}{c}\text { Estimated } \\
\text { Wildife } \\
\text { NOAELc } \\
(\mathrm{mg} / \mathrm{kg} \cdot \mathrm{d})\end{array}$} & \multicolumn{3}{|c|}{ Toxicological Benchmarks } \\
\hline & & & & & $\begin{array}{c}\text { Diet }^{d} \\
\text { (mg/kg) }\end{array}$ & $\begin{array}{l}\text { Water } \\
\text { (mg/L) }\end{array}$ & $\begin{array}{l}\text { Aquatic } \\
\text { Feeding } \\
\text { Species } \\
\text { (mg/L) }\end{array}$ \\
\hline \multirow[t]{8}{*}{ Methanol } & Rat & 50 & $\begin{array}{l}\text { Short-tailed } \\
\text { Shrew }\end{array}$ & 141.385 & 235.641 & 642.657 & \\
\hline & & & Little Brown Bat & 177.723 & 533.168 & 1110.766 & \\
\hline & & & $\begin{array}{l}\text { White-footed } \\
\text { Mouse }\end{array}$ & 124.599 & 806.226 & 415.328 & \\
\hline & & & Meadow Vole & 99.123 & 872.278 & 726.898 & \\
\hline & & & Cottontail Rabbit & 33.296 & 168.585 & 344.436 & \\
\hline & & & Mink & 35.360 & 258.102 & 357.172 & $2.95 \mathrm{e}+02$ \\
\hline & & & Red Fox & 21.526 & 215.255 & 254.907 & \\
\hline & & & Whitetail Deer & 9.340 & 303.282 & 142.624 & \\
\hline \multirow[t]{8}{*}{ Methoxychlor } & Rat & 4 & $\begin{array}{l}\text { Short-tailed } \\
\text { Shrew }\end{array}$ & 11.311 & 18.851 & 51.413 & \\
\hline & & & Little Brown Bat & 14.218 & 42.653 & 88.861 & \\
\hline & & & $\begin{array}{l}\text { White-footed } \\
\text { Mouse }\end{array}$ & 9.968 & 64.498 & 33.226 & \\
\hline & & & Meadow Vole & 7.930 & 69.782 & 58.152 & \\
\hline & & & Cottontail Rabbit & 2.664 & 13.487 & 27.555 & \\
\hline & & & Mink & 2.829 & 20.648 & 28.574 & \\
\hline & & & Red Fox & 1.722 & 17.220 & 20.393 & \\
\hline & & & Whitetail Deer & 0.747 & 24.263 & 11.410 & \\
\hline
\end{tabular}


Table 12. (continued)

\begin{tabular}{|c|c|c|c|c|c|c|c|}
\hline \multirow{2}{*}{$\begin{array}{l}\text { Contaminant } \\
\text { and Form }\end{array}$} & \multirow{2}{*}{ Test Species } & \multirow{2}{*}{$\begin{array}{c}\text { Test } \\
\text { Species } \\
\text { NOAEL: } \\
(\mathbf{m g} / \mathbf{k g} \cdot \mathbf{d})\end{array}$} & \multirow{2}{*}{$\begin{array}{l}\text { Endpoint } \\
\text { Species }^{b}\end{array}$} & \multirow{2}{*}{$\begin{array}{c}\text { Estimated } \\
\text { Wildlife } \\
\text { NOAEL } \\
\left(\mathrm{mg} / \mathrm{kg}^{\circ} \mathrm{d}\right)\end{array}$} & \multicolumn{3}{|c|}{ Toxicological Benchmarks } \\
\hline & & & & & $\begin{array}{c}\text { Diet }^{d} \\
(\mathrm{mg} / \mathbf{k g})\end{array}$ & $\begin{array}{c}\text { Water } \\
\cdot(\mathrm{mg} / \mathrm{L})\end{array}$ & $\begin{array}{l}\text { Aquatic } \\
\text { Feeding } \\
\text { Species' } \\
\text { (mg/L) }\end{array}$ \\
\hline \multirow[t]{8}{*}{ Methylene Chloride } & Rat & 5.85 & $\begin{array}{l}\text { Short-tailed } \\
\text { Shrew }\end{array}$ & 16.542 & 27.570 & 75.191 & \\
\hline & & & Little Brown Bat & 20.794 & 62.381 & 129.960 & \\
\hline & & & $\begin{array}{l}\text { White-footed } \\
\text { Mouse }\end{array}$ & 14.578 & 94.328 & 48.593 & \\
\hline & & & Meadow Vole & 11.597 & 102.057 & 85.047 & \\
\hline & & & Cottontail Rabbit & 3.896 & 19.724 & 40.299 & \\
\hline & & & Mink & 4.137 & 30.198 & 41.789 & $5.06 \mathrm{e}+00$ \\
\hline & & & Red Fox & 2.518 & 25.185 & 29.824 & \\
\hline & & & Whitetail Deer & 1.093 & 35.484 & 16.687 & \\
\hline \multirow[t]{8}{*}{ Methyl Ethyl Ketone } & Rat & 1771 & $\begin{array}{l}\text { Short-tailed } \\
\text { Shrew }\end{array}$ & 5007.839 & 8346.398 & 22762.905 & \\
\hline & & & $\begin{array}{c}\text { White-footed } \\
\text { Mouse }\end{array}$ & 4413.279 & 28556.510 & 14710.930 & \\
\hline & & & Little Brown Bat & 0.000 & 0.000 & 0.000 & \\
\hline & & & Meadow Vole & 3510.919 & 30896.087 & 25746.739 & \\
\hline & & & Cottontail Rabbit & 1179.327 & 5971.274 & 12199.930 & \\
\hline & & & Mink & 1252.451 & 9141.980 & 12651.022 & $5.38 \mathrm{e}+03$ \\
\hline & & & Red Fox & 762.433 & 7624.332 & 9028.814 & \\
\hline & & & Whitetail Deer & 330.823 & 10742.235 & 5051.754 & \\
\hline
\end{tabular}


68

Table 12. (continued)

\begin{tabular}{|c|c|c|c|c|c|c|c|}
\hline \multirow{2}{*}{$\begin{array}{l}\text { Contaminant } \\
\text { and Form }\end{array}$} & \multirow{2}{*}{ Test Species } & \multirow{2}{*}{$\begin{array}{c}\text { Test } \\
\text { Species } \\
\text { NOAEL' } \\
(\mathbf{m g} / \mathbf{k g} \bullet \mathbf{d})\end{array}$} & \multirow{2}{*}{$\begin{array}{l}\text { Endpoint } \\
\text { Species }^{b}\end{array}$} & \multirow{2}{*}{$\begin{array}{c}\text { Estimated } \\
\text { Wildlife } \\
\text { NOAEL } \\
(\mathrm{mg} / \mathrm{kg} \cdot \mathrm{d})\end{array}$} & \multicolumn{3}{|c|}{ Toxicological Benchmarks } \\
\hline & & & & & $\begin{array}{c}\text { Diet }^{d} \\
(\mathrm{mg} / \mathrm{kg})\end{array}$ & $\begin{array}{l}\text { Water }^{e} \\
\text { (mg/L) }\end{array}$ & $\begin{array}{l}\text { Aquatic } \\
\text { Feeding } \\
\text { Species } \\
\text { (mg/L) }\end{array}$ \\
\hline \multirow[t]{8}{*}{ 4-Methyl 2-Pentanone } & Rat & 25 & $\begin{array}{l}\text { Short-tailed } \\
\text { Shrew }\end{array}$ & 70.692 & 117.820 & 321.328 & \\
\hline & & & Little Brown Bat & 88.861 & 266.584 & 555.383 & \\
\hline & & & $\begin{array}{l}\text { White-footed } \\
\text { Mouse }\end{array}$ & 62.299 & 403.113 & 207.664 & \\
\hline & & & Meadow Vole & 49.561 & 436.139 & 363.449 & \\
\hline & & & Cottontail Rabbit & 16.648 & 84.292 & 172.218 & \\
\hline & & & Mink & 17.680 & 129.051 & 178.586 & $2.37 e+01$ \\
\hline & & & Red Fox & 10.763 & 107.628 & 127.454 & \\
\hline & & & Whitetail Deer & 4.670 & 151.641 & 71.312 & \\
\hline Nickel & Rat & 40 & $\begin{array}{l}\text { Short-tailed } \\
\text { Shrew }\end{array}$ & 113.108 & 188.513 & 514.125 & \\
\hline \multirow{7}{*}{$\begin{array}{c}\text { Nickel Sulfate } \\
\text { Hexahydrate }\end{array}$} & & & Little Brown Bat & 142.178 & 426.534 & 888.613 & \\
\hline & & & $\begin{array}{c}\text { White-footed } \\
\text { Mouse }\end{array}$ & 99.679 & 644.980 & 332.263 & \\
\hline & & & Meadow Vole & 79.298 & 697.822 & 581.519 & \\
\hline & & & Cottontail Rabbit & 26.636 & 134.868 & 275.549 & \\
\hline & & & Mink & 28.288 & 206.482 & 285.737 & $1.93 e+00$ \\
\hline & & & Red Fox & 17.220 & 172.204 & 203.926 & \\
\hline & & & Whitetail Deer & 7.472 & 242.625 & 114.099 & \\
\hline
\end{tabular}


Table 12. (continued)

\begin{tabular}{|c|c|c|c|c|c|c|c|}
\hline \multirow{2}{*}{$\begin{array}{l}\text { Contaminant } \\
\text { and Form }\end{array}$} & \multirow{2}{*}{ Test Species } & \multirow{2}{*}{$\begin{array}{c}\text { Test } \\
\text { Species } \\
\text { NOAEL } \\
(\mathbf{m g} / \mathbf{k g} \cdot \mathbf{d})\end{array}$} & \multirow{2}{*}{$\begin{array}{l}\text { Endpoint } \\
\text { Species }^{b}\end{array}$} & \multirow{2}{*}{$\begin{array}{c}\text { Estimated } \\
\text { Wildlife } \\
\text { NOAELc } \\
\left(\mathrm{mg} / \mathrm{kg}^{*} \mathrm{~d}\right)\end{array}$} & \multicolumn{3}{|c|}{ Toxicological Benchmarks } \\
\hline & & & & & $\begin{array}{c}\text { Diet }^{d} \\
(\mathrm{mg} / \mathbf{k g})\end{array}$ & $\begin{array}{r}\text { Water } \\
\text { - (mg/L) }\end{array}$ & $\begin{array}{l}\text { Aquatic } \\
\text { Feeding } \\
\text { Species } \\
\text { (mg/L) }\end{array}$ \\
\hline Nickel & $\begin{array}{l}\text { Mallard } \\
\text { Duckling }\end{array}$ & 77.4 & American Robin & 166.325 & 137.710 & 1208.209 & \\
\hline \multirow[t]{8}{*}{ Nickel Sulfate } & & & $\begin{array}{l}\text { American } \\
\text { Woodcock }\end{array}$ & 121.787 & 160.758 & 1205.687 & \\
\hline & & & Wild Turkey & 39.955 & 1331.822 & 1219.668 & \\
\hline & & & $\begin{array}{c}\text { Belted } \\
\text { Kingfisher }\end{array}$ & 134.065 & 264.554 & 1240.097 & $2.49 e+00$ \\
\hline & & & $\begin{array}{c}\text { Great Blue } \\
\text { Heron }\end{array}$ & 53.534 & 304.632 & 1209.315 & $2.87 e+00$ \\
\hline & & & Barred Owl & 79.648 & 1220.255 & 1215.063 & \\
\hline & & & Bam Owl & 91.819 & 684.601 & 1222.502 & \\
\hline & & & Cooper's Hawk & 93.645 & 1209.129 & 1209.129 & \\
\hline & & & Red-tailed Hawk & 68.627 & 84.916 & 1207.401 & \\
\hline Niobium & mouse & 0.1166 & $\begin{array}{c}\text { Short-tailed } \\
\text { Shrew }\end{array}$ & 0.147 & 0.244 & 0.666 & \\
\hline \multirow[t]{7}{*}{ Sodium Niobate } & & & Little Brown Bat & 0.184 & 0.553 & 1.152 & \\
\hline & & & $\begin{array}{c}\text { White-footed } \\
\text { Mouse }\end{array}$ & 0.129 & 0.836 & 0.431 & \\
\hline & & & Meadow Vole & 0.103 & 0.904 & 0.754 & \\
\hline & & & Cottontail Rabbit & 0.035 & 0.175 & 0.357 & \\
\hline & & & Mink & 0.037 & 0.268 & 0.370 & \\
\hline & & & Red Fox & 0.022 & 0.223 & 0.264 & \\
\hline & & & Whitetail Deer & 0.010 & 0.314 & 0.148 & \\
\hline
\end{tabular}


70

Table 12. (continued)

\begin{tabular}{|c|c|c|c|c|c|c|c|}
\hline \multirow{2}{*}{$\begin{array}{l}\text { Contaminant } \\
\text { and Form }\end{array}$} & \multirow{2}{*}{ Test Species } & \multirow{2}{*}{$\begin{array}{c}\text { Test } \\
\text { Species } \\
\text { NOAEL" } \\
\left(\mathbf{m g} / \mathbf{k g}^{*} \mathrm{~d}\right)\end{array}$} & \multirow{2}{*}{$\begin{array}{l}\text { Endpoint } \\
\text { Species }^{b}\end{array}$} & \multirow{2}{*}{$\begin{array}{c}\text { Estimated } \\
\text { Wildlife } \\
\text { NOAEL } \\
\left(\mathbf{m g} / \mathbf{k g}^{\circ} \mathrm{d}\right)\end{array}$} & \multicolumn{3}{|c|}{ Toxicological Benchmarks } \\
\hline & & & & & $\begin{array}{c}\text { Diet }^{d} \\
(\mathrm{mg} / \mathrm{kg})\end{array}$ & $\begin{array}{l}\text { Watere } \\
\text { (mg/L) }\end{array}$ & $\begin{array}{l}\text { Aquatic } \\
\text { Feeding } \\
\text { Species } \\
\text { (mg/L) }\end{array}$ \\
\hline Nitrate & Guinea Pig & 507 & $\begin{array}{l}\text { Short-tailed } \\
\text { Shrew }\end{array}$ & 1928.780 & 3214.634 & 8767.182 & \\
\hline \multirow[t]{7}{*}{ Potassium Nitrate } & & & Little Brown Bat & 2424.499 & 7273.498 & 15153.121 & \\
\hline & & & $\begin{array}{l}\text { White-footed } \\
\text { Mouse }\end{array}$ & 1699.783 & 10998.599 & 5665.945 & \\
\hline & & & Meadow Vole & 1352.240 & 11899.712 & 9916.427 & \\
\hline & & & Cottontail Rabbit & 454.216 & 2299.829 & 4698.789 & \\
\hline & & & Mink & 482.385 & 3521.059 & 4872.577 & \\
\hline & & & Red Fox & 293.649 & 2936.493 & 3477.426 & \\
\hline & & & Whitetail Deer & 127.414 & 4137.299 & 1945.649 & \\
\hline \multirow[t]{8}{*}{$\begin{array}{l}\text { 1,2,3,4,8-Pentachloro } \\
\text { Dibenzofuran }\end{array}$} & Rat & 0.048 & $\begin{array}{c}\text { Short-tailed } \\
\text { Shrew }\end{array}$ & 0.136 & 0.226 & 0.617 & \\
\hline & & & Litule Brown Bat & 0.171 & 0.512 & 1.066 & \\
\hline & & & $\begin{array}{l}\text { White-footed } \\
\text { Mouse }\end{array}$ & 0.120 & 0.774 & 0.399 & \\
\hline & & & Meadow Vole & 0.095 & 0.837 & 0.698 & \\
\hline & & & Cottontail Rabbit & 0.032 & 0.162 & 0.331 & \\
\hline & & & Mink & 0.034 & 0.248 & 0.343 & \\
\hline & & & Red Fox & 0.021 & 0.207 & 0.245 & \\
\hline & & & Whitetail Deer & 0.009 & 0.291 & 0.137 & \\
\hline
\end{tabular}


71

Table 12. (continued)

\begin{tabular}{|c|c|c|c|c|c|c|c|}
\hline \multirow[b]{2}{*}{$\begin{array}{l}\text { Contaminant } \\
\text { and Form }\end{array}$} & \multirow{2}{*}{ Test Species } & \multirow{2}{*}{$\begin{array}{c}\text { Test } \\
\text { Species } \\
\text { NOAEL" } \\
\left(\mathbf{m g} / \mathbf{k g}^{\bullet} \mathbf{d}\right)\end{array}$} & \multirow{2}{*}{$\begin{array}{l}\text { Endpoint } \\
\text { Species }^{\mathbf{b}}\end{array}$} & \multirow{2}{*}{$\begin{array}{c}\text { Estimated } \\
\text { Wildlife } \\
\text { NOAEL } \\
\left(\mathrm{mg} / \mathbf{k g}^{\mathrm{c}} \mathrm{d}\right)\end{array}$} & \multicolumn{3}{|c|}{ Toxicological Benchmarks } \\
\hline & & & & & $\underset{(\mathbf{m g} / \mathbf{k g})}{\operatorname{Diet}^{d}}$ & $\begin{array}{r}\text { Water } \\
.(\mathbf{m g} / \mathbf{L})\end{array}$ & $\begin{array}{l}\text { Aquatic } \\
\text { Feeding } \\
\text { Species } \\
\text { (mg/L) }\end{array}$ \\
\hline \multirow[t]{8}{*}{$\begin{array}{l}\text { 1,2,3,7,8-Pentachloro } \\
\text { Dibenzofuran } \\
\end{array}$} & Rat & 0.00016 & $\begin{array}{l}\text { Short-tailed } \\
\text { Shrew }\end{array}$ & 0.00045 & 0.00075 & 0.00205 & \\
\hline & & & Litule Brown Bat & 0.0005687 & 0.0017061 & 0.0035544 & \\
\hline & & & $\begin{array}{l}\text { White-footed } \\
\text { Mouse }\end{array}$ & 0.0004 & 0.00259 & 0.00133 & \\
\hline & & & Meadow Vole & 0.00032 & 0.00282 & 0.00235 & \\
\hline & & & Cottontail Rabbit & 0.00011 & 0.00056 & 0.00114 & \\
\hline & & & Mink & 0.00011 & 0.0008 & 0.00111 & \\
\hline & & & Red Fox & 0.00007 & 0.0007 & 0.00083 & \\
\hline & & & Whitetail Deer & 0.00003 & 0.00097 & 0.00046 & \\
\hline \multirow[t]{8}{*}{$\begin{array}{l}\text { 2,3,4,7,8-Pentachloro } \\
\text { Dibenzofuran }\end{array}$} & Rat & 0.000016 & $\begin{array}{l}\text { Short-tailed } \\
\text { Shrew }\end{array}$ & 0.0000452 & 0.0000753 & 0.0002055 & \\
\hline & & & Little Brown Bat & 0.0000567 & 0.00017 & 0.00035 & \\
\hline & & & $\begin{array}{l}\text { White-footed } \\
\text { Mouse }\end{array}$ & 0.0000399 & 0.0002582 & 0.000133 & \\
\hline & & & Meadow Vole & 0.0000317 & 0.000279 & 0.0002325 & \\
\hline & & & Cottontail Rabbit & 0.0000107 & 0.0000542 & 0.0001107 & \\
\hline & & & Mink & 0.0000113 & 0.0000825 & 0.0001141 & \\
\hline & & & Red Fox & 0.0000069 & 0.000069 & 0.0000817 & \\
\hline & & & Whitetail Deer & 0.000003 & 0.0000974 & 0.0000458 & \\
\hline
\end{tabular}


72

Table 12. (continued)

\begin{tabular}{|c|c|c|c|c|c|c|c|}
\hline \multirow{2}{*}{$\begin{array}{l}\text { Contaminant } \\
\text { and Form }\end{array}$} & \multirow{2}{*}{ Test Species } & \multirow{2}{*}{$\begin{array}{c}\text { Test } \\
\text { Species } \\
\text { NOAEL' } \\
(\mathrm{mg} / \mathrm{kg} \bullet \mathrm{d})\end{array}$} & \multirow{2}{*}{$\begin{array}{l}\text { Endpoint } \\
\text { Species }^{b}\end{array}$} & \multirow{2}{*}{$\begin{array}{c}\text { Estimated } \\
\text { Wildlife } \\
\text { NOAEL } \\
\left(\mathrm{mg} / \mathrm{kg}^{\circ} \mathrm{d}\right)\end{array}$} & \multicolumn{3}{|c|}{ Toxicological Benchmarks } \\
\hline & & & & & $\begin{array}{c}\text { Diet }^{d} \\
(\mathrm{mg} / \mathrm{kg})\end{array}$ & $\begin{array}{l}\text { Watere } \\
\text { (mg/L) }\end{array}$ & $\begin{array}{l}\text { Aquatic } \\
\text { Feeding } \\
\text { Species } \\
\text { (mg/L) }\end{array}$ \\
\hline \multirow[t]{9}{*}{ Pentachloronitrobenzene } & Chicken & 7.07 & American Robin & 18.836 & 15.595 & 136.827 & \\
\hline & & & $\begin{array}{l}\text { American } \\
\text { Woodcock }\end{array}$ & .13 .792 & 18.206 & 136.542 & \\
\hline & & & Wild Turkey & 4.525 & 150.827 & 138.125 & \\
\hline & & & $\begin{array}{c}\text { Belted } \\
\text { Kingfisher }\end{array}$ & 15.182 & 29.960 & 140.438 & $1.16 \mathrm{e}-02$ \\
\hline & & & $\begin{array}{l}\text { Great Blue } \\
\text { Heron }\end{array}$ & 6.063 & 34.499 & 136.953 & $1.34 \mathrm{e}-02$ \\
\hline & & & Barred Owl & 9.020 & 138.192 & 137.604 & \\
\hline & & & Barn Owl & 10.398 & 77.530 & 138.446 & \\
\hline & & & Cooper's Hawk & 10.605 & 136.931 & 136.931 & \\
\hline & & & Red-tailed Hawk & 7.772 & 9.617 & 136.736 & \\
\hline Selenium & mouse & 0.075 & $\begin{array}{l}\text { Short-tailed } \\
\text { Shrew }\end{array}$ & 0.094 & 0.157 & 0.429 & \\
\hline \multirow[t]{7}{*}{ Selanate } & & & Litule Brown Bat & 0.119 & 0.356 & 0.741 & \\
\hline & & & $\begin{array}{l}\text { White-footed } \\
\text { Mouse }\end{array}$ & 0.083 & 0.538 & 0.277 & \\
\hline & & & Meadow Vole & 0.066 & 0.582 & 0.485 & \\
\hline & & & Cottontail Rabbit & 0.022 & 0.112 & 0.230 & \\
\hline & & & Mink & 0.024 & 0.172 & 0.238 & $6.62 e-05$ \\
\hline & & & Red Fox & 0.014 & 0.144 & 0.170 & \\
\hline & & & Whitetail Deer & 0.006 & 0.202 & 0.095 & \\
\hline
\end{tabular}


73

Table 12. (continued)

\begin{tabular}{|c|c|c|c|c|c|c|c|}
\hline \multirow{2}{*}{$\begin{array}{l}\text { Contaminant } \\
\text { and Form }\end{array}$} & \multirow{2}{*}{ Test Species } & \multirow{2}{*}{$\begin{array}{c}\text { Test } \\
\text { Species } \\
\text { NOAEL' } \\
\left(\mathrm{mg} / \mathrm{kg}^{\circ} \mathrm{d}\right)\end{array}$} & \multirow{2}{*}{$\begin{array}{l}\text { Endpoint } \\
\text { Species }^{\mathrm{b}}\end{array}$} & \multirow{2}{*}{$\begin{array}{c}\text { Estimated } \\
\text { Wildlife } \\
\text { NOAELc } \\
\left(\mathrm{mg} / \mathrm{kg}^{\circ} \mathrm{d}\right)\end{array}$} & \multicolumn{3}{|c|}{ Toxicological Benchmarks } \\
\hline & & & & & $\underset{(\mathbf{m g} / \mathbf{k g})}{\operatorname{Diet}^{\mathrm{d}}}$ & $\cdot \begin{array}{c}\text { Watere } \\
\cdot(\mathrm{mg} / \mathrm{L})\end{array}$ & $\begin{array}{l}\text { Aquatic } \\
\text { Feeding } \\
\text { Species } \\
\text { (mg/L) }\end{array}$ \\
\hline Selenium & mallard duck & 0.5 & American Robin & 1.165 & 0.965 & 8.465 & \\
\hline \multirow[t]{8}{*}{ Sodium Selanite } & & & $\begin{array}{l}\text { American } \\
\text { Woodcock }\end{array}$ & 0.853 & 1.126 & 8.447 & \\
\hline & & & Wild Turkey & 0.280 & 9.331 & 8.545 & \\
\hline & & & $\begin{array}{c}\text { Belted } \\
\text { Kingfisher }\end{array}$ & 0.939 & 1.853 & 8.688 & $7.13 \mathrm{e}-04$ \\
\hline & & & $\begin{array}{l}\text { Great Blue } \\
\text { Heron }\end{array}$ & 0.375 & 2.134 & 8.473 & $8.21 \mathrm{e}-04$ \\
\hline & & & Barred Owl & 0.558 & 8.549 & 8.513 & \\
\hline & & & Barn Owl & 0.643 & 4.796 & 8.565 & \\
\hline & & & Cooper's Hawk & 0.656 & 8.471 & 8.471 & \\
\hline & & & Red-tailed Hawk & 0.481 & 0.595 & 8.459 & \\
\hline Selenium & Maliard Duck & 0.4 & American Robin & 0.932 & 0.772 & 6.772 & \\
\hline \multirow[t]{8}{*}{ Selanomethionine } & & & $\begin{array}{l}\text { American } \\
\text { Woodcock }\end{array}$ & 0.683 & 0.901 & 6.758 & \\
\hline & & & Wild Turkey & 0.224 & 7.465 & 6.836 & \\
\hline & & & $\begin{array}{c}\text { Belted } \\
\text { Kingfisher }\end{array}$ & 0.751 & 1.483 & 6.950 & \\
\hline & & & $\begin{array}{c}\text { Great Blue } \\
\text { Heron }\end{array}$ & 0.300 & 1.707 & 6.778 & \\
\hline & & & Barred Owl & 0.446 & 6.839 & 6.810 & \\
\hline & & & Barn Owl & 0.515 & 3.837 & 6.852 & \\
\hline & & & Cooper's Hawk & 0.525 & 6.777 & 6.777 & \\
\hline & & & Red-tailed Hawk & 0.385 & 0.476 & 6.767 & \\
\hline
\end{tabular}


74

Table 12. (continued)

\begin{tabular}{|c|c|c|c|c|c|c|c|}
\hline \multirow[b]{2}{*}{$\begin{array}{c}\text { Contaminant } \\
\text { and Form }\end{array}$} & \multirow{2}{*}{ Test Species } & \multirow{2}{*}{$\begin{array}{c}\text { Test } \\
\text { Species } \\
\text { NOAEL" } \\
\left(\mathbf{m g} / \mathbf{k g} \bullet^{\circ} \mathbf{d}\right)\end{array}$} & \multirow{2}{*}{$\begin{array}{l}\text { Endpoint } \\
\text { Species }\end{array}$} & \multirow{2}{*}{$\begin{array}{c}\text { Estimated } \\
\text { Wildife } \\
\text { NOAEL } \\
\left(m g / k^{c} \bullet^{-d}\right)\end{array}$} & \multicolumn{3}{|c|}{ Toxicological Benchmarks } \\
\hline & & & & & $\begin{array}{c}\text { Diet }^{d} \\
(\mathrm{mg} / \mathrm{kg})\end{array}$ & $\begin{array}{c}\text { Watere } \\
\text { (mg/L) }\end{array}$ & $\begin{array}{l}\text { Aquatic } \\
\text { Feeding } \\
\text { Species } \\
\text { (mg/L) }\end{array}$ \\
\hline Strontium (stable) & Rat & 263 & $\begin{array}{l}\text { Short-tailed } \\
\text { Shrew }\end{array}$ & 743.682 & 1239.471 & 3380.375 & \\
\hline \multirow[t]{7}{*}{ Strontium Chloride } & & & Little Brown Bat & 934.820 & 2804.461 & 5842.627 & \\
\hline & & & $\begin{array}{l}\text { White-footed } \\
\text { Mouse }\end{array}$ & 655.388 & 4240.747 & 2184.627 & \\
\hline & & & Meadow Vole & 521.384 & 4588.182 & 3823.485 & \\
\hline & & & Cottontail Rabbit & 175.134 & 886.756 & 1811.734 & \\
\hline & & & Mink & 185.994 & 1357.618 & 1878.723 & \\
\hline & & & Red Fox & 113.224 & 1132.241 & 1340.812 & \\
\hline & & & Whitetail Deer & 49.128 & 1595.261 & 750.204 & \\
\hline \multirow[t]{8}{*}{$\begin{array}{l}\text { 2,3,7,8-Tetrachloro } \\
\text { Dibenzodioxin }\end{array}$} & Rat & 0.000001 & $\begin{array}{c}\text { Short-tailed } \\
\text { Shrew }\end{array}$ & 0.0000028 & 0.00000467 & $\begin{array}{c}0.0000127 \\
3\end{array}$ & \\
\hline & & & Little Brown Bat & 0.000003554 & 0.000010662 & $\begin{array}{c}0.0000222 \\
13\end{array}$ & \\
\hline & & & $\begin{array}{l}\text { White-footed } \\
\text { Mouse }\end{array}$ & 0.0000025 & 0.00001618 & $\begin{array}{c}0.0000083 \\
3 \\
\end{array}$ & \\
\hline & & & Meadow Vole & 0.000002 & 0.0000176 & $\begin{array}{c}0.0000146 \\
7\end{array}$ & \\
\hline & & & Cottontail Rabbit & 0.0000007 & 0.00000354 & $\begin{array}{c}0.0000072 \\
4\end{array}$ & \\
\hline & & & Mink & 0.0000007 & 0.00000511 & $\begin{array}{c}0.0000070 \\
7\end{array}$ & $5.89 \mathrm{e}-11$ \\
\hline & & & Red Fox & 0.000000431 & 0.00000431 & 0.0000051 & \\
\hline & & & Whitetail Deer & 0.000000187 & 0.00000607 & $\begin{array}{c}0.0000028 \\
6\end{array}$ & \\
\hline
\end{tabular}


Table 12. (continued)

\begin{tabular}{|c|c|c|c|c|c|c|c|}
\hline \multirow[b]{2}{*}{$\begin{array}{l}\text { Contaminant } \\
\text { and Form }\end{array}$} & \multirow{2}{*}{ Test Species } & \multirow{2}{*}{$\begin{array}{c}\text { Test } \\
\text { Species } \\
\text { NOAEL } \\
\left(\mathrm{mg} / \mathrm{kg}^{\circ} \mathrm{d}\right)\end{array}$} & \multirow{2}{*}{$\begin{array}{l}\text { Endpoint } \\
\text { Species }^{b}\end{array}$} & \multirow{2}{*}{$\begin{array}{l}\text { Estimated } \\
\text { Wildlife } \\
\text { NOAEL } \\
\left(\mathrm{mg} / \mathrm{kg}^{\circ} \mathrm{d}\right)\end{array}$} & \multicolumn{3}{|c|}{ Toxicological Benchmarks } \\
\hline & & & & & $\begin{array}{c}\text { Diet }^{d} \\
\text { (mg/kg) }\end{array}$ & $\begin{array}{r}\text { Watere } \\
\text { (mg/L) }\end{array}$ & $\begin{array}{l}\text { Aquatic } \\
\text { Feeding } \\
\text { Species } \\
\text { (mg/L) }\end{array}$ \\
\hline \multirow[t]{9}{*}{$\begin{array}{l}\text { 2,3,7,8-Tetrachloro } \\
\text { Dibenzodioxin } \\
\end{array}$} & $\begin{array}{l}\text { Ring-necked } \\
\text { Pheasant }\end{array}$ & 0.000014 & American Robin & 0.0000326 & 0.000027 & 0.0002368 & \\
\hline & & & $\begin{array}{l}\text { American } \\
\text { Woodcock }\end{array}$ & 0.0000239 & 0.0000315 & 0.0002366 & \\
\hline & & & Wild Turkey & 0.0000078 & 0.00026 & 0.0002381 & \\
\hline & & & $\begin{array}{c}\text { Belted } \\
\text { Kingfisher }\end{array}$ & 0.0000263 & 0.0000519 & 0.0002433 & $5.99 \mathrm{e}-10$ \\
\hline & & & $\begin{array}{c}\text { Great Blue } \\
\text { Heron }\end{array}$ & 0.0000105 & 0.0000598 & 0.0002372 & $6.89 \mathrm{e}-10$ \\
\hline & & & Barred Owl & 0.0000156 & 0.000239 & 0.000238 & \\
\hline & & & Barn Owl & 0.000018 & 0.0001342 & 0.0002397 & \\
\hline & & & Cooper's Hawk & 0.0000184 & 0.0002376 & 0.0002376 & \\
\hline & & & Red-tailed Hawk & 0.0000135 & 0.0000167 & 0.0002375 & \\
\hline \multirow{9}{*}{$\begin{array}{l}\text { 2,3,7,8-Tetrachloro } \\
\text { Dibenzofuran }\end{array}$} & Chicken & $1.0 \mathrm{e}-06$ & American Robin & 0.0000012 & 0.000001 & 0.0000087 & \\
\hline & & & $\begin{array}{l}\text { American } \\
\text { Woodeock }\end{array}$ & 0.0000009 & 0.0000012 & 0.0000089 & \\
\hline & & & Wild Turkey & 0.0000003 & 0.00001 & 0.0000092 & \\
\hline & & & $\begin{array}{c}\text { Belted } \\
\text { Kingfisher } \\
\end{array}$ & 0.0000009 & 0.0000018 & 0.0000083 & \\
\hline & & & $\begin{array}{c}\text { Great Blue } \\
\text { Heron }\end{array}$ & 0.0000004 & 0.0000023 & 0.000009 & \\
\hline & & & Barred Owl & 0.0000006 & 0.0000092 & 0.0000092 & \\
\hline & & & Bam Owl & 0.0000006 & 0.0000045 & 0.000008 & \\
\hline & & & Cooper's Hawk & 0.0000007 & 0.000009 & 0.000009 & \\
\hline & & & Red-tailed Hawk & 0.0000005 & 0.0000006 & 0.0000088 & \\
\hline
\end{tabular}


76

Table 12. (continued)

\begin{tabular}{|c|c|c|c|c|c|c|c|}
\hline \multirow{2}{*}{$\begin{array}{l}\text { Contaminant } \\
\text { and Form }\end{array}$} & \multirow{2}{*}{ Test Species } & \multirow{2}{*}{$\begin{array}{c}\text { Test } \\
\text { Species } \\
\text { NOAEL } \\
\left(\mathrm{mg} / \mathrm{kg}^{*} \mathrm{~d}\right)\end{array}$} & \multirow{2}{*}{$\begin{array}{l}\text { Endpoint } \\
\text { Species }^{b}\end{array}$} & \multirow{2}{*}{$\begin{array}{c}\text { Estimated } \\
\text { Wildlife } \\
\text { NOAEL } \\
\left(\mathrm{mg} / \mathrm{kg}^{\cdot} \cdot \mathrm{d}\right)\end{array}$} & \multicolumn{3}{|c|}{ Toxicological Benchmarks } \\
\hline & & & & & $\begin{array}{c}\text { Diet }^{d} \\
\text { (mg/kg) }\end{array}$ & $\begin{array}{c}\text { Watere } \\
(\mathrm{mg} / \mathrm{L})\end{array}$ & $\begin{array}{l}\text { Aquatic } \\
\text { Feeding } \\
\text { Species } \\
\text { (mg/L) }\end{array}$ \\
\hline \multirow[t]{8}{*}{$\begin{array}{l}\text { 1,1,2,2-Tetrachloroethy } \\
\text { ene }\end{array}$} & mouse & 1.4 & $\begin{array}{l}\text { Short-tailed } \\
\text { Shrew }\end{array}$ & 1.760 & 2.933 & 7.999 & \\
\hline & & & Little Brown Bat & 2.212 & 6.636 & 13.826 & \\
\hline & & & $\begin{array}{l}\text { White-footed } \\
\text { Mouse }\end{array}$ & 1.551 & 10.035 & 5.170 & \\
\hline & & & Meadow Vole & 1.234 & 10.857 & 9.048 & \\
\hline & & & Cottontail Rabbit & 0.414 & 2.098 & 4.287 & \\
\hline & & & Mink & 0.440 & 3.213 & 4.446 & $1.42 \mathrm{e}-02$ \\
\hline & & & Red Fox & 0.268 & 2.679 & 3.173 & \\
\hline & & & Whitetail Deer & 0.116 & 3.775 & 1.775 & \\
\hline Thallium & Rat & 0.0074 & $\begin{array}{l}\text { Short-tailed } \\
\text { Shrew }\end{array}$ & 0.021 & 0.035 & 0.096 & \\
\hline \multirow[t]{7}{*}{ Thallium Sulfate } & & & Little Brown Bat & 0.027 & 0.080 & 0.167 & \\
\hline & & & $\begin{array}{l}\text { White-footed } \\
\text { Mouse }\end{array}$ & 0.019 & 0.121 & 0.062 & \\
\hline & & & Meadow Vole & 0.015 & 0.131 & 0.109 & \\
\hline & & & Cottontail Rabbit & 0.005 & 0.025 & 0.052 & \\
\hline & & & Mink & 0.005 & 0.039 & 0.054 & $1.12 \mathrm{e}-03$ \\
\hline & & & Red Fox & 0.003 & 0.032 & 0.038 & \\
\hline & & & Whitetail Deer & 0.001 & 0.045 & 0.021 & \\
\hline
\end{tabular}


Table 12. (continued)

\begin{tabular}{|c|c|c|c|c|c|c|c|}
\hline \multirow{2}{*}{$\begin{array}{l}\text { Contaminant } \\
\text { and Form }\end{array}$} & \multirow{2}{*}{ Test Species } & \multirow{2}{*}{$\begin{array}{c}\text { Test } \\
\text { Species } \\
\text { NOAEL: } \\
(\mathbf{m g} / \mathbf{k g} \cdot \mathbf{d})\end{array}$} & \multirow{2}{*}{$\begin{array}{l}\text { Endpoint } \\
\text { Species }^{b}\end{array}$} & \multirow{2}{*}{$\begin{array}{c}\text { Estimated } \\
\text { Wildlife } \\
\text { NOAEL } \\
\left(\mathrm{mg} / \mathrm{kg}^{\circ} \cdot \mathrm{d}\right)\end{array}$} & \multicolumn{3}{|c|}{ Toxicological Benchmarks } \\
\hline & & & & & $\begin{array}{c}\text { Diet }^{d} \\
\text { (mg/kg) }\end{array}$ & $\begin{array}{c}\text { Watere } \\
\text {. (mg/L) }\end{array}$ & $\begin{array}{l}\text { Aquatic } \\
\text { Feeding } \\
\text { Species } \\
\text { (mg/L) }\end{array}$ \\
\hline \multirow[t]{8}{*}{ Toluene } & Rat & 25.98 & $\begin{array}{l}\text { Short-tailed } \\
\text { Shrew }\end{array}$ & 32.657 & 54.429 & 148.441 & \\
\hline & & & Little Brown Bat & 41.050 & 123.151 & 256.566 & \\
\hline & & & $\begin{array}{l}\text { White-footed } \\
\text { Mouse }\end{array}$ & 28.780 & 186.223 & 95.933 & \\
\hline & & & Meadow Vole & 22.895 & 201.479 & 167.900 & \\
\hline & & & Cottontail Rabbit & 7.691 & 38.940 & 79.558 & \\
\hline & & & Mink & 8.168 & 59.617 & 82.501 & $8.44 \mathrm{e}-01$ \\
\hline & & & Red Fox & 4.972 & 49.721 & 58.880 & \\
\hline & & & Whitetail Deer & 2.157 & 70.053 & 32.944 & \\
\hline \multirow[t]{8}{*}{ Toxaphene } & Rat & 8 & $\begin{array}{c}\text { Short-tailed } \\
\text { Shrew }\end{array}$ & 22.622 & 37.703 & 102.825 & \\
\hline & & & Little Brown Bat & 28.436 & 85.307 & 177.723 & \\
\hline & & & $\begin{array}{c}\text { White-footed } \\
\text { Mouse }\end{array}$ & 19.936 & 128.996 & 66.453 & \\
\hline & & & Meadow Vole & 15.860 & 139.564 & 116.304 & \\
\hline & & & Cottontail Rabbit & 5.327 & 26.974 & 55.110 & \\
\hline & & & Mink & 5.658 & 41.296 & 57.147 & $1.02 e-02$ \\
\hline & & & Red Fox & 3.444 & 34.441 & 40.785 & \\
\hline & & & Whitetail Deer & 1.494 & 48.525 & 22.820 & \\
\hline
\end{tabular}


78

Table 12. (continued)

\begin{tabular}{|c|c|c|c|c|c|c|c|}
\hline \multirow{2}{*}{$\begin{array}{l}\text { Contaminant } \\
\text { and Form }\end{array}$} & \multirow{2}{*}{ Test Species } & \multirow{2}{*}{$\begin{array}{c}\text { Test } \\
\text { Species } \\
\text { NOAEL" } \\
(m g / k g \cdot d)\end{array}$} & \multirow{2}{*}{$\begin{array}{l}\text { Endpoint } \\
\text { Species }^{b}\end{array}$} & \multirow{2}{*}{$\begin{array}{c}\text { Estimated } \\
\text { Wildlife } \\
\text { NOAELc } \\
\left(\mathrm{mg} / \mathrm{kg}^{\bullet} \mathrm{d}\right)\end{array}$} & \multicolumn{3}{|c|}{ Toxicological Benchmarks } \\
\hline & & & & & $\begin{array}{c}\text { Diet }^{d} \\
(\mathrm{mg} / \mathrm{kg})\end{array}$ & $\begin{array}{l}\text { Watere } \\
\text { (mg/L) }\end{array}$ & $\begin{array}{l}\text { Aquatic } \\
\text { Feeding } \\
\text { Species } \\
\text { (mg/L) }\end{array}$ \\
\hline \multirow[t]{8}{*}{ 1,1,1-Trichloroethane } & mouse & 1000 & $\begin{array}{l}\text { Short-tailed } \\
\text { Shrew }\end{array}$ & 1322.610 & 2204.350 & 6011.864 & \\
\hline & & & Little Brown Bat & 1662.540 & 4987.620 & 10390.875 & \\
\hline & & & $\begin{array}{l}\text { White-footed } \\
\text { Mouse }\end{array}$ & 1165.580 & 7541.988 & 3885.267 & \\
\hline & & & Meadow Vole & 927.260 & 8159.888 & 6799.907 & \\
\hline & & & Cottontail Rabbit & 311.470 & 1577.063 & 3222.103 & \\
\hline & & & Mink & 330.780 & 2414.453 & 3341.212 & $5.17 e+01$ \\
\hline & & & Red Fox & 201.360 & 2013.600 & 2384.526 & \\
\hline & & & Whitetail Deer & 87.370 & 2837.014 & 1334.164 & \\
\hline \multirow[t]{8}{*}{ Trichloroethylene } & mouse & 0.7 & $\begin{array}{c}\text { Short-tailed } \\
\text { Shrew }\end{array}$ & 0.880 & 1.467 & 4.000 & \\
\hline & & & Little Brown Bat & 1.106 & 3.318 & 6.913 & \\
\hline & & & $\begin{array}{l}\text { White-footed } \\
\text { Mouse }\end{array}$ & 0.775 & 5.018 & 2.585 & \\
\hline & & & Meadow Vole & 0.617 & 5.429 & 4.524 & \\
\hline & & & Cottontail Rabbit & 0.207 & 1.049 & 2.144 & \\
\hline & & & Mink & 0.220 & 1.606 & 2.223 & $3.88 e-02$ \\
\hline & & & Red Fox & 0.134 & 1.340 & 1.586 & \\
\hline & & & Whitetail Deer & 0.058 & 1.888 & 0.888 & \\
\hline
\end{tabular}


Table 12. (continued)

\begin{tabular}{|c|c|c|c|c|c|c|c|}
\hline \multirow{2}{*}{$\begin{array}{l}\text { Contaminant } \\
\text { and Form }\end{array}$} & \multirow{2}{*}{ Test Species } & \multirow{2}{*}{$\begin{array}{c}\text { Test } \\
\text { Species } \\
\text { NOAEL" } \\
(\mathbf{m g} / \mathbf{k g} \bullet \mathbf{d})\end{array}$} & \multirow{2}{*}{$\begin{array}{l}\text { Endpoint } \\
\text { Species }^{b}\end{array}$} & \multirow{2}{*}{$\begin{array}{c}\text { Estimated } \\
\text { Wildlife } \\
\text { NOAELc } \\
(\mathrm{mg} / \mathrm{kg} \cdot \mathrm{d})\end{array}$} & \multicolumn{3}{|c|}{ Toxicological Benchmarks } \\
\hline & & & & & $\begin{array}{c}\text { Diet }^{d} \\
\text { (mg/kg) }\end{array}$ & $\begin{array}{l}\text { Watere } \\
\text { (mg/L) }\end{array}$ & $\begin{array}{l}\text { Aquatic } \\
\text { Feeding } \\
\text { Species } \\
\text { (mg/L) }\end{array}$ \\
\hline Uranium & mouse & 3.07 & $\begin{array}{c}\text { Short-tailed } \\
\text { Shrew }\end{array}$ & 3.772 & 6.287 & 17.146 & \\
\hline \multirow[t]{7}{*}{ Uranyl Acetate } & & & Little Brown Bat & 4.742 & 14.225 & 29.635 & \\
\hline & & & $\begin{array}{l}\text { White-footed } \\
\text { Mouse }\end{array}$ & 3.324 & 21.510 & 11.081 & \\
\hline & & & Mesdow Vole & 2.645 & 23.273 & 19.394 & \\
\hline & & & Cottontail Rabbit & 0.888 & 4.498 & 9.190 & \\
\hline & & & Mink & 0.943 & 6.886 & 9.529 & \\
\hline & & & Red Fox & 0.574 & 5.743 & 6.801 & \\
\hline & & & Whitetail Deer & 0.249 & 8.092 & 3.805 & \\
\hline Uranium & black duck & 16 & American Robin & 40.138 & 33.233 & 291.570 & \\
\hline \multirow[t]{8}{*}{ depleted metal } & & & $\begin{array}{l}\text { American } \\
\text { Woodcock }\end{array}$ & 29.390 & 38.795 & 290.962 & \\
\hline & & & Wild Turikey & 9.642 & 321.403 & 294.337 & \\
\hline & & & $\begin{array}{c}\text { Belted } \\
\text { Kingfisher }\end{array}$ & 32.353 & 63.843 & 299.265 & \\
\hline & & & $\begin{array}{c}\text { Great Blue } \\
\text { Heron }\end{array}$ & 12.919 & 73.515 & 291.838 & \\
\hline & & & Barred Owl & 19.221 & 294.477 & 293.224 & \\
\hline & & & Barn Owl & 22.158 & 165.211 & 295.019 & \\
\hline & & & Cooper's Hawk & 22.599 & 291.791 & 291.791 & \\
\hline & & & Red-tailed Hawk & 16.561 & 20.492 & 291.375 & \\
\hline
\end{tabular}


80

Table 12. (continued)

\begin{tabular}{|c|c|c|c|c|c|c|c|}
\hline \multirow{2}{*}{$\begin{array}{l}\text { Contaminant } \\
\text { and Form }\end{array}$} & \multirow{2}{*}{ Test Species } & \multirow{2}{*}{$\begin{array}{c}\text { Test } \\
\text { Species } \\
\text { NOAEL" } \\
\left(\mathrm{mg} / \mathrm{kg}^{*} \mathrm{~d}\right)\end{array}$} & \multirow{2}{*}{$\begin{array}{l}\text { Endpoint } \\
\text { Species }^{b}\end{array}$} & \multirow{2}{*}{$\begin{array}{c}\text { Estimated } \\
\text { Wildlife } \\
\text { NOAELc } \\
(\mathrm{mg} / \mathrm{kg} \cdot \mathrm{d})\end{array}$} & \multicolumn{3}{|c|}{ Toxicological Benchmarks } \\
\hline & & & & & $\begin{array}{c}\text { Diet }^{d} \\
\text { (mg/kg) }\end{array}$ & $\begin{array}{l}\text { Watere } \\
\text { (mg/L) }\end{array}$ & $\begin{array}{l}\text { Aquatic } \\
\text { Feeding } \\
\text { Species } \\
\text { (mg/L) }\end{array}$ \\
\hline Vanadium & Rat & 0.21 & $\begin{array}{c}\text { Short-lailed } \\
\text { Shrew }\end{array}$ & 0.538 & 0.897 & 2.447 & \\
\hline \multirow[t]{7}{*}{ Sodium Metavanadate } & & & Little Brown Bat & 0.677 & 2.030 & 4.229 & \\
\hline & & & $\begin{array}{l}\text { White-footed } \\
\text { Mouse }\end{array}$ & 0.474 & 3.070 & 1.581 & \\
\hline & & & Meadow Vole & 0.377 & 3.321 & 2.768 & \\
\hline & & & Cottontail Rabbit & 0.127 & 0.642 & 1.311 & \\
\hline & & & Mink & 0.135 & 0.983 & 1.360 & \\
\hline & & & Red Fox & 0.082 & 0.820 & 0.971 & \\
\hline & & & Whitetail Deer & 0.036 & 1.155 & 0.543 & \\
\hline Vanadium & Mallard Duck & 11.38 & American Robin & 27.932 & 23.126 & 202.902 & \\
\hline \multirow[t]{8}{*}{ Vanadyl Sulfate } & & & $\begin{array}{l}\text { American } \\
\text { Woodcock }\end{array}$ & 20.452 & 26.997 & 202.478 & \\
\hline & & & Wild Turkey & 6.710 & 223.663 & 204.828 & \\
\hline & & & $\begin{array}{c}\text { Belted } \\
\text { Kingfisher }\end{array}$ & 22.514 & 44.428 & 208.256 & \\
\hline & & & $\begin{array}{c}\text { Great Blue } \\
\text { Heron }\end{array}$ & 8.990 & 51.159 & 203.089 & \\
\hline & & & Barred Owl & 13.376 & 204.924 & 204.052 & \\
\hline & & & Barn Owl & 15.420 & 114.969 & 205.302 & \\
\hline & & & Cooper's Hawk & 15.726 & 203.057 & 203.057 & \\
\hline & & & Red-tailed Hawk & 11.525 & 14.260 & 202.766 & \\
\hline
\end{tabular}


81

Table 12. (continued)

\begin{tabular}{|c|c|c|c|c|c|c|c|}
\hline \multirow[b]{2}{*}{$\begin{array}{l}\text { Contaminant } \\
\text { and Form }\end{array}$} & \multirow{2}{*}{ Test Species } & \multirow{2}{*}{$\begin{array}{c}\text { Test } \\
\text { Species } \\
\text { NOAEL' } \\
\left(\mathbf{m g} / \mathbf{k g}{ }^{\circ} \mathrm{d}\right)\end{array}$} & \multirow{2}{*}{$\begin{array}{l}\text { Endpoint } \\
\text { Species }\end{array}$} & \multirow{2}{*}{$\begin{array}{c}\text { Estimated } \\
\text { Wildlife } \\
\text { NOAEL } \\
(\mathrm{mg} / \mathrm{kg} \cdot \mathrm{d})\end{array}$} & \multicolumn{3}{|c|}{ Toxicological Benchmarks } \\
\hline & & & & & $\underset{(\mathbf{m g} / \mathbf{k g})}{\mathrm{Diet}^{\mathrm{d}}}$ & $\begin{array}{l}\text { Water } \\
(\mathrm{mg} / \mathrm{L})\end{array}$ & $\begin{array}{l}\text { Aquatic } \\
\text { Feeding } \\
\text { Species } \\
\text { (mg/L) }\end{array}$ \\
\hline \multirow[t]{8}{*}{ Vinyl Chloride } & Rat & 0.17 & $\begin{array}{l}\text { Short-tailed } \\
\text { Shrew }\end{array}$ & 0.481 & 0.801 & 2.185 & \\
\hline & & & Little Brown Bat & 0.604 & 1.813 & 3.777 & \\
\hline & & & $\begin{array}{l}\text { White-footed } \\
\text { Mouse }\end{array}$ & 0.424 & 2.741 & 1.412 & \\
\hline & & & Meadow Vole & 0.337 & 2.966 & 2.471 & \\
\hline & & & Cottontail Rabbit & 0.113 & 0.573 & 1.171 & \\
\hline & & & Mink & 0.120 & 0.878 & 1.214 & $1.24 \mathrm{e}-01$ \\
\hline & & & Red Fox & 0.073 & 0.732 & 0.867 & \\
\hline & & & Whitetail Deer & 0.032 & 1.031 & 0.485 & \\
\hline \multirow[t]{8}{*}{ Xylene (mixed isomers) } & mouse & 2.06 & $\begin{array}{l}\text { Short-tailed } \\
\text { Shrew }\end{array}$ & 2.589 & 4.316 & 11.770 & \\
\hline & & & Little Brown Bat & 3.255 & 9.765 & 20.344 & \\
\hline & & & $\begin{array}{l}\text { White-footed } \\
\text { Mouse }\end{array}$ & 2.282 & 14.766 & 7.607 & \\
\hline & & & Meadow Vole & 1.815 & 15.976 & 13.313 & \\
\hline & & & Cottontail Rabbit & 0.610 & 3.088 & 6.308 & \\
\hline & & & Mink & 0.648 & 4.727 & 6.542 & $2.96 \mathrm{e}-02$ \\
\hline & & & Red Fox & 0.394 & 3.942 & 4.669 & \\
\hline & & & Whitetail Deer & 0.171 & 5.555 & 2.612 & \\
\hline
\end{tabular}


82

Table 12. (continued)

\begin{tabular}{|c|c|c|c|c|c|c|c|}
\hline \multirow[b]{2}{*}{$\begin{array}{l}\text { Contaminant } \\
\text { and Form }\end{array}$} & \multirow{2}{*}{ Test Species } & \multirow{2}{*}{$\begin{array}{c}\text { Test } \\
\text { Species } \\
\text { NOAEL: } \\
\left(\mathrm{mg} / \mathrm{kg} \bullet^{\circ} \mathrm{d}\right)\end{array}$} & \multirow{2}{*}{$\begin{array}{l}\text { Endpoint } \\
\text { Species }^{b}\end{array}$} & \multirow{2}{*}{$\begin{array}{c}\text { Estimated } \\
\text { Wildiffe } \\
\text { NOAELc } \\
\left(\mathrm{mg} / \mathrm{kg}^{\circ} \mathrm{d}\right)\end{array}$} & \multicolumn{3}{|c|}{ Toxicological Benchmarks } \\
\hline & & & & & $\begin{array}{c}\text { Diet }^{d} \\
(\mathrm{mg} / \mathrm{kg})\end{array}$ & $\begin{array}{l}\text { Watere } \\
\text { (mg/L) }\end{array}$ & $\begin{array}{l}\text { Aquatic } \\
\text { Feeding } \\
\text { Species } \\
\text { (mg/L) }\end{array}$ \\
\hline Zine & Rat & 160 & $\begin{array}{c}\text { Short-tailed } \\
\text { Shrew }\end{array}$ & 452.430 & 754.051 & 2056.502 & \\
\hline \multirow[t]{7}{*}{ Zinc Oxide } & & & Little Brown Bat & 568.712 & 1706.136 & 3554.450 & \\
\hline & & & $\begin{array}{l}\text { White-footed } \\
\text { Mouse }\end{array}$ & 398.715 & 2579.922 & 1329.051 & \\
\hline & & & Meadow Vole & 317.192 & 2791.290 & 2326.075 & \\
\hline & & & Cottontail Rabbit & 106.546 & 539.471 & 1102.196 & \\
\hline & & & Mink & 113.152 & 825.927 & 1142.949 & $8.54 e-01$ \\
\hline & & & Red Fox & 68.882 & 688.816 & 815.703 & \\
\hline & & & Whitetail Deer & 29.888 & 970.501 & 456.398 & \\
\hline Zinc & Mallard Duck & 3 & American Robin & 6.992 & 5.789 & 50.788 & \\
\hline \multirow[t]{8}{*}{ Zinc Carbonate } & & & $\begin{array}{l}\text { American } \\
\text { Woodcock }\end{array}$ & 5.119 & 6.758 & 50.682 & \\
\hline & & & Wild Turkey & 1.680 & 55.985 & 51.270 & \\
\hline & & & $\begin{array}{l}\text { Belted } \\
\text { Kingfisher }\end{array}$ & 5.636 & 11.121 & 52.129 & $1.15 \mathrm{e}-02$ \\
\hline & & & $\begin{array}{c}\text { Great Blue } \\
\text { Heron }\end{array}$ & 2.250 & 12.806 & 50.835 & $1.33 \mathrm{e}-02$ \\
\hline & & & Barred Owl & 3.348 & 51.295 & 51.077 & \\
\hline & & & Barn Owl & 3.860 & 28.778 & 51.389 & \\
\hline & & & Cooper's Hawk & 3.936 & 50.827 & 50.827 & \\
\hline & & & Red-tailed Hawk & 2.885 & 3.570 & 50.754 & \\
\hline
\end{tabular}


Table 12. (continued)

\begin{tabular}{|c|c|c|c|c|c|c|c|}
\hline \multirow[b]{2}{*}{$\begin{array}{l}\text { Contaminant } \\
\text { and Form }\end{array}$} & \multirow{2}{*}{ Test Species } & \multirow{2}{*}{$\begin{array}{c}\text { Test } \\
\text { Species } \\
\text { NOAEL• } \\
(\mathbf{m g} / \mathbf{k g} \bullet \mathbf{d})\end{array}$} & \multirow{2}{*}{$\begin{array}{l}\text { Endpoint } \\
\text { Species }^{b}\end{array}$} & \multirow{2}{*}{$\begin{array}{c}\text { Estimated } \\
\text { Wildife } \\
\text { NOAELc } \\
(m g / k g \bullet d)\end{array}$} & \multicolumn{3}{|c|}{ Toxicological Benchmarks } \\
\hline & & & & & $\begin{array}{c}\text { Diet }^{d} \\
(\mathbf{m g} / k g)\end{array}$ & $\begin{array}{l}\text { Watere } \\
\text { (mg/L) }\end{array}$ & $\begin{array}{l}\text { Aquatic } \\
\text { Feeding } \\
\text { Species' } \\
\text { (mg/L) }\end{array}$ \\
\hline Zirconium & mouse & 1.738 & $\begin{array}{l}\text { Short-tailed } \\
\text { Shrew }\end{array}$ & 2.185 & 3.641 & 9.930 & \\
\hline \multirow[t]{7}{*}{ Zirconium Sulfate } & & & Little Brown Bat & 2.746 & 8.239 & 17.164 & \\
\hline & & & $\begin{array}{l}\text { White-footed } \\
\text { Mouse }\end{array}$ & 1.925 & 12.458 & 6.418 & \\
\hline & & & Meadow Vole & 1.532 & 13.479 & 11.232 & \\
\hline & & & Cottontail Rabbit & 0.514 & 2.605 & 5.322 & \\
\hline & & & Mink & 0.546 & 3.988 & 5.519 & \\
\hline & & & Red Fox & 0.333 & 3.326 & 3.939 & \\
\hline & & & Whitetail Deer & 0.144 & 4.686 & 2.204 & \\
\hline
\end{tabular}

- See Appendix A for NOAEL derivation, study duration and study endpoint.

bee Appendix B for body weights, food and water consumption rates.

- Calculated using Equation 4.

- Calculated using Equation 8.

- Calculated using Equation 19.

${ }^{f}$ Combined food and water benchmark for aquatic-feeding species. Calculated using Equation 26. 
84

Table 13. Use of benchmarks in a screening assessment

\begin{tabular}{|c|c|c|c|c|c|c|c|c|c|}
\hline \multirow[t]{3}{*}{ Analyte } & \multicolumn{3}{|c|}{$\begin{array}{l}\text { Contaminant Concentrations } \\
\text { in Media }\end{array}$} & \multicolumn{2}{|c|}{$\begin{array}{l}\text { Benchmarks for } \\
\text { Meadow Vole }\end{array}$} & \multicolumn{4}{|c|}{$\begin{array}{c}\text { Comparison of Media Concentrations to } \\
\text { Benchmarks }\end{array}$} \\
\hline & \multirow{2}{*}{$\begin{array}{l}\text { Water } \\
\text { (mg/L) }\end{array}$} & \multirow{2}{*}{$\begin{array}{c}\text { Soil } \\
\text { (mg/kg) }\end{array}$} & \multirow{2}{*}{$\begin{array}{c}\text { Estimated } \\
\text { in Plants } \\
(\mathrm{mg} / \mathrm{kg})\end{array}$} & \multirow{2}{*}{$\begin{array}{l}\text { Water } \\
(\mathrm{mg} / \mathrm{L})\end{array}$} & \multirow{2}{*}{$\begin{array}{c}\text { Diet } \\
(\mathrm{mg} / \mathrm{kg})\end{array}$} & \multicolumn{2}{|c|}{ Water } & \multicolumn{2}{|c|}{ Diet } \\
\hline & & & & & & $H Q^{b}$ & $\begin{array}{l}\text { Retain as } \\
\text { COPC }\end{array}$ & $H Q^{b}$ & $\begin{array}{l}\text { Retain as } \\
\text { COP }\end{array}$ \\
\hline Arsenic & 0.038 & 131 & 5.24 & 0.814 & 0.977 & 0.047 & No & 5.36 & YES \\
\hline Lead & 0.069 & 18.8 & 0.85 & 116.3 & 139.56 & 0.0006 & No & 0.006 & No \\
\hline Mercurye & 0.005 & 0.71 & 0.64 & 0.465 & 0.558 & 0.011 & No & 1.15 & YES \\
\hline Selenium & 0.02 & 14.8 & 0.37 & 0.485 & 0.582 & 0.041 & NO & 0.64 & No \\
\hline
\end{tabular}

- Estimates using plant uptake factors for foliage from Baes et al. (1984).

- $\mathrm{HQ}=$ Hazard Quotient = Media Concentration/Benchmark.

c Mercury assumed to be in the form of Methyl Mercury.

Table 14. Use of benchmarks in a baseline assessment

\begin{tabular}{|c|c|c|c|c|c|c|c|c|c|}
\hline \multirow[t]{2}{*}{ Analyte } & \multicolumn{3}{|c|}{$\begin{array}{c}\text { Contaminant Concentrations } \\
\text { in Media }\end{array}$} & \multicolumn{4}{|c|}{$\begin{array}{c}\text { Contaminant Exposure } \\
(\mathrm{mg} / \mathrm{kg} \text { bw/d) }\end{array}$} & \multirow{2}{*}{$\begin{array}{c}\text { NOAEL } \\
\text { for } \\
\text { Meadow } \\
\text { Vole }\end{array}$} & \multirow[t]{2}{*}{ HQ } \\
\hline & $\begin{array}{l}\text { Water } \\
(\mathrm{mg} / \mathrm{L})\end{array}$ & $\begin{array}{c}\text { Soil } \\
(\mathrm{mg} / \mathrm{kg})\end{array}$ & $\begin{array}{l}\text { Plants } \\
\text { (mg/kg) }\end{array}$ & Water & Soil & Diet & Total & & \\
\hline Arsenic & 0.038 & 131 & 1.77 & 0.0052 & 0.298 & 0.201 & 0.504 & 0.111 & 4.54 \\
\hline Lead & 0.069 & 18.8 & 1.07 & 0.0094 & 0.043 & 0.122 & 0.174 & 15.86 & 0.01 \\
\hline Mercuryb & 0.005 & 0.71 & 0.06 & 0.0007 & 0.0016 & 0.007 & 0.0093 & 0.063 & 0.15 \\
\hline Selenium & 0.02 & 14.8 & 23.61 & 0.003 & 0.034 & 2.68 & 2.717 & 0.066 & 41.1 \\
\hline
\end{tabular}

- $H Q=$ Hazard Quotient = Total Exposure/Benchmark.

bercury assumed to be in the form of Methyl Mercury. 


\section{REFERENCES}

Abiola, F.A. 1992. "Ecotoxicity of organochloride insecticides: effects of endosulfan on birds reproduction and evaluation of its induction effects in partridge, Perdix perdix L." Rev. Vet. Med. 143: 443-450.

Alexander, G.R. 1977. "Food of vertebrate predators on trout waters in north central lower Michigan." Mich. Acad. 10: 181-195.

Alumot, E. (Olomucki), E. Nachtomi, E. Mandel, and P. Holstein. 1976a. "Tolerance and acceptable daily intake of chlorinated fumigants in the rat diet." Fd. Cosmet. Toxicol. 14: 105-110.

Alumot, E., M. Meidler, and P. Holstein. 1976b. "Tolerance and acceptable daily intake of ethylene dichloride in the chicken diet." Fd. Cosmet. Toxicol. 14: 111-114.

Ambrose, A.M., P.S. Larson, J.F. Borzelleca, and G.R. Hennigar, Jr. 1976. "Long-term toxicologic assessment of nickel in rats and dogs." J. Food Sci. Tech. 13: 181-187.

Anderson, D.W., R.W. Risebrough, L.A. Woods, Jr., L.R. DeWeese, and W.G. Edgecomb. 1975. "Brown pelicans: improved reproduction off the southern California coast." Science 190: 806-808.

Anthony, E. L. P. and T. H. Kunz. 1977. "Feeding strategies of the little brown bat, Myotis lucifugus, in Southern New Hampshire." Ecology. 58: 775-786.

Aulerich, R.J. and R.K. Ringer. 1977. "Current status of PCB toxicity, including reproduction in mink." Arch. Environ. Contam. Toxicol. 6: 279.

Aulerich, R.J. and R.K. Ringer. 1980. Toxicity of the polychlorinated biphenyl Aroclor 1016 to mink. Environmental Research Laboratory, Office of Research and Development.

Aulerich, R.J., R.K. Ringer, M.R. Bleavins, et al. 1982. "Effects of supplemental dietary copper on growth, reproductive performance and kit survival of standard dark mink and the acute toxicity of copper to mink." J. Animal Sci. 55: 337-343.

Aulerich, R.J., A.C. Napolitano, S.J. Bursian, B.A. Olson, and J.R. Hochstein. 1987. "Chronic toxicity of dietary fluorine in mink." J. Anim. Sci. 65: 1759-1767.

ATSDR (Agency for Toxic Substances and Disease Registry). 1989. Toxicological profile for selected PCBs (Aroclor-1260, -1254, -1248, -1242,-1232, -1221, and-1016). ATSDR/TP$88 / 21$. 
Azar, A., H.J. Trochimowicz, and M.E. Maxwell. 1973. "Review of lead studies in animals carried out at Haskell Laboratory: two-year feeding study and response to hemorrhage study." In: Environmental Health Aspects of Lead: Proceedings, International Symposium, D. Barth et al., eds. Commission of European Communities. pp. 199-210.

Baes, C.F.,II, R.D. Sharp, A.L. Sjoren, and R.W. Shor. 1994. A review and analysis of parameters for assessing transport of environmentally released radionuclides through agriculture. Oak Ridge National Laboratory, Oak Ridge, TN. ORNL-5786

Baroni, C., G.J. VanEsch, and U. Saffiotti. 1963. "Carcinogenesis tests of two inorganic arsenicals." Arch. Environ. Health. 7: 668-674.

Barrett, G.W., and K.L. Stueck. 1976. "Caloric ingestion rate and assimilation efficiency of the short-tailed shrew, Blarina brevicauda." Ohio J. Sci. 76: 25-26.

Barsotti, D.A., R.J. Marlar and J.R. Allen. 1976. "Reproductive dysfuction in Rhesus monkeys exposed to low levels of polychlorinated biphenyls (Aroclor 1248)." Fd. Cosmet. Toxicol. 14: 99-103.

Baxley, M.N., R.D. Hood, G.C. Vedel, W.P. Harrison, and G.M. Szczech. 1981. "Prenatal toxicity of orally administered sodium arsenite in mice." Bull. Environ. Contam. Toxicol. 26: 749-756.

Beyer, W.N., E. Conner, and S. Gerould. 1994. "Survey of soil ingestion by wildlife." J. Wildl. Mgmt. 58: 375-382.

Blakely, B.R., C.S. Sisodia, and T.K. Mukkur. 1980. "The effect of methyl mercury, tetrethyl lead, and sodium arsenite on the humoral immune response in mice." Toxicol. Appl. Pharmacol. 52: 245-254.

Bleavins, M.R., R.J. Aulerich, and R.K. Ringer. 1980. "Polychlorinated biphenyls (Aroclors 1016 and 1242): Effect on survival and reproduction in mink and ferrets." Arch. Environ. Contam. Toxicol. 9: 627-635.

Bleavins, M.R. and R.J. Aulerich. 1981. "Feed consumption and food passage time in mink (Mustela vison) and European ferrets (Mustela putorius furo)." Lab. Anim. Sci. 31: 268269.

Bleavins, M.R., R.J. Aulerich, and R.K. Ringer. 1984. "Effects of chronic dietary hexachlorobenzene exposure on the reproductive performance and survivability of mink and European ferrets." Arch. Environ. Contam. Toxicol. 13: 357-365.

Buben, J.A. and E.J. O'Flaherty. 1985. "Delineation of the role of metabolism in the hepatotoxicity of trichloroethylene and perchloroethylene: a dose-effect study." Toxicol. Appl. Pharmacol. 78: 105-122. 
Buckner, C.H. 1964. "Metabolism, food capacity, and feeding behavior in four species of shrews." Can. J. Zool. 42: 259-279.

Burt, W.H. and R.P. Grossenheider. 1976. A field guide to the mammals of America north of Mexico. Third Edition. Houghton Mifflin Co., Boston.

Byron, W.R., G.W. Bierbower, J.B. Brower, and W.H. Hansen. 1967. "Pathological changes in rats and dogs from two-year feeding of sodium arsenite or sodium arsenate." Toxicol. Appl. Pharmacol. 10: 132-147.

Cain, B.W. and E.A. Pafford. 1981. "Effects of dietary nickel on survival and growth of Mallard ducklings." Arch. Environm. Contam. Toxicol. 10: 737-745.

Calder, W.A. and E.J. Braun. 1983. "Scaling of osmotic regulation in mammals and birds." Am. J. Physiol. 224: Rr601-R606.

Carriere, D., K. Fischer, D. Peakall, and P. Angehrn. 1986. "Effects of dietary aluminum in combination with reduced calcium and phosphorus on the ring dove (Streptopelia risoria)." Water, Air, and Soil Poll. 30: 757-764.

Chakravarty, S. and P. Lahiri. 1986. "Effect of lindane on eggshell characteristics and calcium level in the domestic duck." Toxicology. 42: 245-258.

Chapman, J.A., J.G. Hockman, and M.M. Ojeda C. 1980. "Sylvilagus floridanus." Mamm. Species. No. 136, pp. 1-8.

Chew, R.M. 1951. "The water exchanges of some small mammals." Ecol. Monogr. 21(3): 215-224.

Collins, W.T. and C.C. Capen. 1980. "Fine structural lesions and hormonal alterations in thyroid glands of perinatal rats exposed in utero and by milk to polychlorinated biphenyls." Am. J. Pathol. 99: 125-142.

Cox, G.E., D.E. Bailey, and K. Morgareidge. 1975. Toxicity studies in rats with 2-butanol including growth, reproduction and teratologic observations. Food and Drug Research Laboratories, Inc., Waverly, NY, Report No. 91MR R 1673.

Dahlgren, R.B., R.L. Linder, and C.W. Carlson. 1972. "Polychlorinated biphenyls: their effects on penned pheasants." Environ. Health Perspect. 1: 89-101.

Dalke,P.D. and P.R. Sime. 1941. "Food habits of the eastern and New England cottontails." J. Wildl. Manage. 5(2): 216-228.

Dark, J., I. Zucker, and G.N. Wade. 1983. "Photoperiodic regulation of body mass, food intake, and reproduction in meadow voles." Am. J. Physiol. 245: R334-R338. 
Dikshith, T.S.S., R.B. Raizada, M.K. Srivastava, and B.S. Kaphalia. 1984. "Response of rats to repeated oral administration of endosulfan." Ind. Health. 22: 295-304.

Domingo, J.L., J.L. Paternain, J.M. Llobet, and J. Corbella. 1986. "Effects of vanadium on reproduction, gestation, parturition and lactation in rats upon oral administration." Life Sci. 39: 819-824.

Dunn, J. S., P. B. Bush, N. H. Booth, R. L. Farrell, D. M. Thomason, and D. D. Goetsch. 1979. "Effect of pentachloronitrobenzene upon egg production, hatchability, and residue accumulation in the tissues of White Leghorn hens." Toxocol. Appl. Pharmacol. 48: 425433.

Dunning, J.B. 1984. Body weights of 686 species of North American birds. West. Bird Banding Assoc. Monogr. No. 1. Eldon Publ. Co. Cave Crk, AZ. 38 pp.

Eisler, M. 1968. "Heptachlor: toxicology and safety evaluation." Ind. Med. Surg., Nov. 840844.

Eisler, R. 1988. Arsenic hazards to fish, wildlife, and invertebrates: a synoptic review. Fish and Wildlife Service, U.S. Department of the Interior. Report No. 85(1.12).

EPA (U. S. Environmental Protection Agency). 1980a. Guidelines and methodology used in the preparation of health effects assessment chapters of the consent decree water quality criteria documents. Fed. Regist. 45(231): 79347-79356.

EPA (U. S. Environmental Protection Agency). 1980b. Ambient water quality criteria for antimony. Office of Water Regulations and Standards, Washington, D.C.

EPA (U. S. Environmental Protection Agency). 1980c. Ambient water quality criteria for beryllium. EPA 440/5-80-024. Office of Water Regulations and Standards, Washington, D.C.

EPA (U. S. Environmental Protection Agency). 1980d. Ambient water quality criteria for thallium. EPA 440/5-80-074. Office of Water Regulations and Standards, Washington, D.C.

EPA (U. S. Environmental Protection Agency). 1985a. Reference values for risk assessment. Prepared by Syracuse Research Corporation, Syracuse, NY for Environmental Criteria and Assessment Office, Cincinnati, $\mathrm{OH}$.

EPA (U. S. Environmental Protection Agency). 1985b. Ambient water quality criteria for Lead - 1984. EPA 440/5-84-027. Office of Water Regulations And Standards, Washington, D.C.

EPA (U. S. Environmental Protection Agency). 1985c. Ambient water quality criteria for cyanide - 1984. EPA 440/5-84-028. Office of Water Regulations And Standards, Washington, D.C. 
EPA (U. S. Environmental Protection Agency). 1985d. Ambient water quality criteria for chromium - 1984. EPA 440/5-84-029. Office of Water Regulations and Standards, Washington, D.C.

EPA (U. S. Environmental Protection Agency). 1985e. Ambient water quality criteria for copper - 1984. EPA 440/5-84-031. Office of Water Regulations and Standards, Washington, D.C.

EPA (U. S. Environmental Protection Agency). 1985f. Ambient water quality criteria for cadmium - 1984. EPA 440/5-84-032. Office of Water Regulations and Standards, Washington, D.C.

EPA (U. S. Environmental Protection Agency). 1985g. Ambient water quality criteria for arsenic - 1984, EPA 440/5-84-033. Office of Water Regulations and Standards, Washington, D.C.

EPA (U. S. Environmental Protection Agency). 1986a. Toxicology Handbook. Government Institutes, Inc., Rockville, MD

EPA (U. S. Environmental Protection Agency). 1986b. Guidelines for carcinogenic risk assessment. Fed. Regist. 51: 33992.

EPA (U. S. Environmental Protection Agency). 1986c. 90-day gavage study in albino rats using acetone. Office of Solid Waste, Washington, D.C.

EPA (U. S. Environmental Protection Agency). 1986d. Rat oral subchronic study with ethyl acetate. Office of Solid Waste, Washington, D.C.

EPA (U. S. Environmental Protection Agency). 1986e. Rat oral subchronic study with methanol. Office of Solid Waste, Washington, D.C.

EPA (U. S. Environmental Protection Agency). 1986f. Ambient water qality criteria for nickel 1986. EPA 440/5-86-004. Office of Water Regulations and Standards, Washington,D.C.

EPA (U. S. Environmental Protection Agency). 1987. Ambient aquatic life water quality criteria document for zinc. EPA/440/5-87-003. Office of Research and Development, Washington, D.C.

EPA (U. S. Environmental Protection Agency). 1988a. Recommendations for and documentation of biological values for use in risk assessment. Environmental Criteria and Assessment Office, Cincinnati, OH. EPA/600/6-87/008.

EPA (U. S. Environmental Protection Agency). 1988b. Methodology for evaluating potential carcinogenicity in support of reportable quantity adjustments pursuant to CERCLA Section 102. OHEA-C-073, External Review Draft. Office of Health and Environmental Assessment, Washington, D.C. 
EPA (U. S. Environmental Protection Agency). 1988c. Ambient water quality criteria for aluminum. EPA/440/5-86-008. Office of Research and Development, Washington, D.C.

EPA (U. S. Environmental Protection Agency). 1989. Water quality criteria to protect wildlife resources. EPA/600/3-89/067. Environmental Research Laboratory, Corvallis, OR.

EPA (U. S. Environmental Protection Agency). 1992. Dermal exposure assessment: principles and applications. Office of Health and Environmental Assessment, Washington, D.C. EPA/600/8-91/011B.

EPA (U. S. Environmental Protection Agency). 1993a. Wildlife exposure factors handbook. Volume 1. Office of Research and Development, Washington, DC. EPA/600/R-93/187a.

EPA (U. S. Environmental Protection Agency). 1993b. Wildlife exposure factors handbook. Volume II. Office of Research and Development, Washington, D.C. EPA/600/R93/187b.

EPA (U. S. Environmental Protection Agency). 1993c. Water quality guidance for the Great Lakes System and correction; proposed rules. Fed. Regist. 58: 20802-21047.

EPA (U. S. Environmental Protection Agency). 1993d. Wildlife criteria portions of the proposed water quality guidance for the Great Lakes system. EPA/822/R-93/006. Office of Science and Technology, Washington, D.C.

EPA (U. S. Environmental Protection Agency). 1993e. Great Lakes water quality initiative criteria documents for the protection of wildlife (proposed): DDT, Mercury, 2,3,7,8-TCDD, PCBs. EPA/822/R-93-007. Office Science and Technology, Washington, D.C.

EPA (U. S. Environmental Protection Agency). 1993f. Health effects assessment summary tables: Annual update. U. S. Environmental Protection Agency. Office of Emergency and Remedial Response. Washington, D.C. OHEA-ECAO-CIN-909.

Feron, V.J., C.F.M. Hendriksen, A.J. Speek, et al. 1981. "Lifespan oral toxicity study of vinyl chloride in rats." Food Cosmet. Toxicol. 13: 633-638.

Fitzhugh, O.G. 1948. "Use of DDT insecticides on food products." Ind. Eng. Chem. 40: 704705.

Formigli, L., R. Scelsi, P. Poggi, C. Gregotti, A. DiNucci, E. Sabbioni, L. Gottardi, and L. Manzo. 1986. "Thallium-induced testicular toxicity in the rat." Environ. Res. 40: 531-539.

Garthoff, L.H., F.E. Cerra, and E.M. Marks. 1981. "Blood chemistry alteration in rats after single and multiple gavage administration of polychlorinated biphenyls." Toxicol. Appl. Pharmacol. 60: 33-44.

Gasaway, W.C. and I.O. Buss. 1972. "Zinc toxicity in the mallard." J. Wildl. Manage. 36: 1107-1117. 
Giavini, E., C. Vismara, and L. Broccia. 1985. "Teratogenesis study of dioxane in rats." Toxicol. Lett. 26: 85-88.

Good, E.E., and G.W. Ware. 1969. "Effects of insecticides on reproduction in the laboratory mouse, IV. Endrin and Dieldrin." Toxicol. Appl. Pharmacol. 14: 201-203.

Gould, Ed. 1955. "The feeding efficiency of insectivorous bats." J. Mammal. 36: 399-407.

Grant, D.L., W.E.J. Phillips, and G.V. Hatina. 1977. "Effects of hexachlorobenzene on reproduction in the rat." Arch. Environ. Contam. Toxicol. 5: 207-216.

Gray, L.E., Jr., J. Ostby, R. Sigmon, J. Ferrell, G. Rehnberg, R. Linder, R. Cooper, J. Goldman, and J. Laskey. 1988. "The development of a protocol to assess reproductive effects of toxicants in the rat." Reprod. Toxicol. 2: 281-287.

Green, D.A. and J.S. Millar. 1987. "Changes in gut dimensions and capacity of Peromyscus maniculatus relative to diet quality and energy needs." Can. J. Zool. 65: 2159-2162.

Harrison, J.W., E.W. Packman, and D.D. Abbott. 1958. "Acute oral toxicity and chemical and physical properties of arsenic trioxides." Arch. Ind. Health. 17: 118-123.

Haseltine, S.D. and L. Sileo. 1983. "Response of American Black ducks to dietary uranium: a proposed substitute for lead shot." $J$. Wildl. Manage. 47: 1124-1129.

Haseltine, S.D., L. Sileo, D.J. Hoffman, and B.D. Mulhern. 1985. "Effects of chromium on reproduction and growth in black ducks."

Hazelton, P.K., R. J. Robel, and A.D. Dayton. 1984. "Preferences and influence of paired food items on energy intake of American robins and gray catbirds." J. Wildl. Manage. 48(1): 198-202.

Heinz, G.H. 1979. "Methyl mercury: reproductive and behavioral effects on three generations of mallard ducks." J. Wildl. Mgmt. 43: 394-401..

Heinz, G.H., D.J. Hoffman, A.J. Krynitsky, and D.M.G. Weller. 1987. "Reproduction in mallards fed selenium." Environ. Toxicol. Chem. 6: 423-433.

Heinz, G.H., D.J. Hoffman, and L.G. Gold. 1989. "Impaired reproduction of mallards fed an organic form of selenium." J. Wildl. Mgmt. 53: 418-428.

Hornshaw, T.C., R.J. Aulerich, and R.K. Ringer. 1986. "Toxicity of o-Cresol to mink and European ferrets." Environ. Toxicol. 5: 713-720.

Hudson, R. H., R. K. Tucker, and M. A. Haegele. 1984. "Handbook of toxicity of pesticides to wildlife." U.S. Fish and Wildl. Serv. Resour. Publ. 153. 90 pp. 
Hurni, H. and H. Ohder. 1973. "Reproduction study with formaldehyde and hexamethylenetetramine in Beagle dogs." Fd. Cosmet. Toxicol. 11: 459-462.

Ivankovic, S. and R. Preussmann. 1975. "Absence of toxic and carcinogenic effects after administration of high doses of chromic oxide pigment in subacute and long-term feeding experiments in rats." $F d$. cosmet. Toxicol. 13: 347-351.

Johnsgard, P.A. 1988. "North American Owls: Biology and Natural History." Smithsonian Institution Press, Washington.

Johnson, D., Jr., A.L. Mehring, Jr., and H.W. Titus. 1960. "Tolerance of chickens for barium." Proc. Soc. Exp. Biol. Med. 104: 436-438.

Kennedy, G.L.,Jr., J.P. Frawley., and J.C. Calandra. 1973. "Multigeneration reproductive effects of three pesticides." Toxicol. Appl. Pharmacol. 25: 589-596.

Knoflach, P., B. Albini, and M.M. Weiser. 1986. "Autoimmune disease induced by oral administration of mercuric chloride in brown-Norway rats." Toxicol. Pathol. 14: 188-193.

Korschgen, L.J. 1967. "Feeding habits and foods." In: The Wild Turkey and Its Management. pp. 137- 198.

Kushlan, J.A. 1978. "Feeding ecology of wading birds." Wading Birds. National Audobon Society. p. 249-297.

Lamb, J.C., IV, R.E. Chapin, J. Teague, A.D. Lawton, and J.R. Reel. 1987. "Reproductive effects of four phthalic acid esters in the mouse." Toxicol. Appl. Pharmacol. 88: 255-269.

Lane, R. W., B. L. Riddle, and J. F. Borzelleca. 1982. " Effects of 1,2-dichloroethane and 1,1,1-trichloroethane in drinking water on reproduction and development in mice." Toxicol. Appl. Pharmacol. 63: 409-421.

Larson, P. S., J. L. Egle, Jr., G. R. Hennigar, R. W. Lane, and J. F. Borzelleca. 1979. "Acute, subchronic, and chronic toxicity of chlordecone." Toxicol. Appl. Pharmacol. 48: $29-41$.

Laskey, J.W., G.L. Rehnberg, J.F. Hein, and S.D. Carter. 1982. "Effects of chronic manganese $\left(\mathrm{Mn}_{3} \mathrm{O}_{4}\right)$ exposure on selected reproductive parameters in rats." J. Toxicol. Environ. Health. 9: 677-687.

Linder, R.E., T.B. Gaines, and R.D. Kimbrough. 1974. "The effect of PCB on rat reproduction." Food Cosmet. Toxicol. 12: 63.

Linzey, A.V. 1987. "Effects of chronic polychlorinated biphenyls exposure on reproductive success of white-footed mice (Peromyscus leucopus)." Arch. Environ. Contamin. Toxicol. 16: $455-460$. 
Lyman, W.J., W.F. Reehl, and D.H. Rosenblatt. 1982. "Handbook of chemical property estimation methods: environmental behavior of organic compounds." McGraw-Hill Book Company, New York.

Mackenzie, R.D., R.U. Byerrum, C.F. Decker, C.A. Hoppert, and R.F. Langham. 1958. "Chronic toxicity studies, II. Hexavalent and trivalent chromium administered in drinking water to rats." Am. Med. Assoc. Arch. Ind. Health. 18: 232-234.

Mackenzie, K.M. and D.M. Angevine. 1981. "Infertility in mice exposed in utero to benzo[a]pyrene." Biol. Reprod. 24: 183-191.

Mankes, R.F., I. Rosenblum, K.F. Benitz, R. Lefevre, and R. Abraham. 1982. "Teratogenic and reproductive effects of ethanol in Long-Evans rats." J. of Toxicol. Environ. Health. 10: 267-276.

Marathe, M.R., and G.P. Thomas. 1986. "Embryotoxicity and teratogenicity of lithium carbonate in Wistar rat." Toxicol. Lett. 34: 115-120.

Marks, T.A., T.A. Ledoux, and J.A. Moore. 1982. "Teratogenicity of a commercial xylene mixture in the mouse." J. Toxico. Environ. Health. 9: 97-105.

Mautz, W.W., H. Silver, J.B. Holter, H.H. Hayes, and W.E. Urban. 1976. "Digestibility and related nutritional data for seven northern deer browse species." J. Wildl. Manage. 40(4): 630-638.

McKinney, J.D., K. Chae, B.N. Gupta, J.A. Moore, and J.A. Goldstein. 1976. "Toxicological assessment of hexachlorobiphenyl and 2,3,7,8-tetrachlorodibenzofuran in chicks. I. Relationship of chemical parameters." Toxicol. Appl. Pharmacol. 36: 65-80.

McLane, M.A.R., and D.L. Hughes. 1980 "Reproductive success of Screech owls fed Aroclor 1248." Arch. Environm. Contam. Toxicol. 9: 661-665.

Mehring, A.L. Jr., J.H. Brumbaugh, A.J. Sutherland, and H.W. Titus. 1960. "The tolerance of growing chickens for dietary copper." Poult. Sci. 39: 713-719.

Mendenhall, V.M., E.E. Klaas, and M.A.R. McLane. 1983. "Breeding success of barn owls (Tyto alba)fed low levels of DDE and dieldrin." Arch. Environ. Contam. Toxicol. 12: 235240.

Menzies, C.A., D.E. Burmaster, J.S. Freshman, and C.A. Callahan. 1992. "Assessment of methods for estimating ecological risk in the terrestrial component: a case study at the Baird and McGuire Superfund site in Holbrook, Massachusetts." Environ. Toxicol. Chem. 11: 245-260.

Merck. 1976. "The Merck Index: an encylopedia of chemicals and drugs." Merck and Co. Inc. Rahway, NJ. 1313pp. 
Merson, M.H. and R.L. Kirkpatrick. 1976. "Reproductive performance of captive white-footed mice fed a polychlorinated biphenyl." Bull. Environ. Contam. Toxicol. 16: 392-398.

Meyers, S.M. and S.M. Schiller. 1986. "TERRE-TOX: a data base for the effects of anthropogenic substances on terrestrial animals." J. Chem. Info. Comp. Sci. 26: 33-36.

Microbiological Associates. 1986. "Subchronic toxicity of methyl isobutyl ketone in SpragueDawley rats." Study No. 5221.0. Preliminary report to Research Triangle Institute, Research Triangle Park, NC.

Murray, F.J., F.A. Smith, K.D. Nitschke, C.G. Humiston, R.J. Kociba, and B.A. Schwetz. 1979. "Three-generation reproduction study of rats given 2,3,7,8-tetrachlorodibenzo-pdioxin (TCDD) in the diet." Toxicol. Appl. Pharmacol. 50: 241-252.

Nagy, K.A. 1987. "Field metabolic rate and food requirement scaling in mammals and birds." Ecol. Monogr. 57: 111-128.

NAS. 1977. "Arsenic." Nat'l. Acad. Aci., Washington, D.C. 332 pp.

Nawrot, P.S. and R.E. Staples. 1979. "Embryofetal toxicity and teratogenicity of benzene and toluene in the mouse." Teratology. 19: 41A

NCA (National Coffe Association). 1982. "24-month chronic toxicity and oncogenicity study of methylene chloride in rats." Final Report. Hazelton Laboratories, Inc., Vienna VA.

NCI (National Cancer Institute). 1978. "Bioassay of Aroclor 1254 for possible carcinogenicity." NCI Carcinogenesis Technical Rep. Series No. 38, NCI-CG-TR-38, DHEW Pub. No. (NIH) 78-838.

Neiger, R.D. and G.D. Osweiler. 1989. "Effect of subacute low level dietary sodium arsenite on dogs." Fund. Appl. Toxicol. 13: 439-451.

Nosek, J.A., S.R. Craven, J.R. Sullivan, S.S. Hurley, and R.E. Peterson. 1992. "Toxicity and reproductive effects of 2,3,7,8-tetrachlorodibenzo-p-dioxin in ring-necked pheasants." $J$. Toxicol. Environ. Health. 35: 187-198.

NRCC. 1978. "Effects of arsenic in the Canadian environment." Natl. Res. Coun. Canada. Publ. No. NRCC 15391. 349 pp.

Ondreicka, R., E. Ginter, and J. Kortus. 1966. "Chronic toxicity of aluminum in rats and mice and its effects on phosphorus metabolism." Brit. J. Indust. Med. 23: 305-313.

Oswald, C., P.Fonken, D. Atkinson, and M. Palladino. 1993. "Lactational water balance and recycling in White-footed mice, Red-backed voles, and gerbils." J. Mammal. 74: 963-970.

Palmer, A.K., D.D. Cozens, E.J.F. Spicer, and A.N. Worden. 1978. "Effects of lindane upon reproductive functions in a 3-generation study in rats." Toxicology. 10: 45-54. 
Palmer, A.K., A.E. Street, F.J.C. Roe, A.N. Worden, and N.J. Van Abbe. 1979. "Safety evaluation of toothpaste containing chloroform, II. Long term studies in rats." J. Environ. Pathol. Toxicol. 2: 821-833.

Paternain, J.L., J.L. Domingo, A. Ortega, and J.M. Llobet. 1989. "The effects of uranium on reproduction, gestation, and postnatal survival in mice." Ecotoxicol. Environ. Saf. 17: 291296.

Pattee, O.H. 1984. "Eggshell thickness and reproduction in American kestrels exposed to chronic dietary lead." Arch Environ. Contam. Toxicol. 13: 29-34.

Pattee, O.H., S.N. Wiemeyer, and D.M. Swineford. 1988. "Effects of dietary fluoride on reproduction in eastern Screech-Owls." Arch. Environ. Contam. Toxicol. 17: 213-218.

Peakall, D.B. 1974. "Effects of di-N-buylphthalate and di-2-ethylhexylphthalate on the eggs of ring doves." Bull. Environ. Contam. Toxicol. 12: 698-702.

Perry, H.M., E.F. Perry, M.N. Erlanger, and S.J. Kopp. 1983. "Cardiovascular effects of chronic barium ingestion." In: Proc. 17th Ann. Conf. Trace Substances in Environ. Health, vol. 17. U. of Missouri Press, Columbia, MO.

Pershagen, G. and M. Vahter. 1979. "Arsenic-a toxicological and epidemiological appraisal," Naturvardsverket Rapp. SNV PM 1128, Liber Tryck, Stockholm. 265 pp.

Peterson, J.A. and A.V. Nebeker. 1992. "Estimation of waterborne selenium concentrations that are toxicity thresholds for wildlife." Arch. Environ. Contam. Toxicol. 23: 154-162.

Poiger, H., N. Pluess, and C. Schlatter. 1989. "Subchronic toxicity of some chlorinated dibenzofurans to rats." Chemosphere. 18: 265-275.

Quast, J.F., C.G. Humiston, C.E. Wade, et al. 1983. "A chronic toxicity and oncogenicity study in rats and subchronic toxicity in dogs on ingested vinylidene chloride." Fund. Appl. Toxicol. 3: 55-62.

Reich, L.M. 1981. "Microtus pennsylvanicus." Mammalian Spec. 159: 1-8.

Revis, N., G. Holdsworth, G. Bingham, A. King, and J. Elmore. 1989. "An assessment of health risk associated with mercury in soil and sediment from East Fork Poplar Creek, Oak Ridge, Tennessee." Oak Ridge Research Institute, Final Report, 58 pp.

Ringer, R.K., R.J. Aulerich and M.R. Bleavins. 1981. "Biological effects of PCBs and PBBs on mink and ferrets; a review." In: Halogenated Hydrocarbons: Health and Ecological Effects. M.A.Q. Khan, ed. Permagon Press, Elmsford, NY, pp. 329-343.

Robertson, I.D., W.E. Harms, and P.J. Ketterer. 1984. "Accidental arsenical toxicity to cattle." Aust. Vet. J. 61: 366-367. 
Sanders, O.T. and R.L. Kirkpatrick. 1975. "Effects of a polychlorinated biphenyl on sleeping times, plasma corticosteroids, and testicular activity of white-footed mice." Environ. Physiol. Biochem. 5: 308-313.

Sargeant, A.B. 1978. "Red fox prey demands and implications to prairie duck production." J. Wildl. Manage. 42(3): 520-527.

Schlesinger, W.H. and G.L. Potter. 1974. "Lead, copper, and cadmium concentrations in small mammals in the Hubbard Brook experimental forest." OIKOS. 25: 148-152.

Schlicker, S.A. and D.H. Cox. 1968. "Maternal dietary zinc, and development and zinc, iron, and copper content of the rat fetus." J. Nutr. 95: 287-294.

Schroeder, H.A. and J.J. Balassa. 1967. "Arsenic, germanium, tin, and vanadium in mice: effects on growth, survival and tissue levels." J. Nutr. 92: 245-252.

Schroeder, H.A., M. Kanisawa, D.V. Frost, and M. Mitchener. 1968a. "Germanium, tin, and arsenic in rats: effects on growth, survival and tissue levels." J. Nutr. 96: 37-45.

Schroeder, H.A., M. Mitchener, J.J. Balassa, M. Kanisawa, and A.P. Nason. 1968b. "Zirconium, niobium, antimony, and fluorine in mice: effects on growth, survival and tissue levels." J. Nutr. 95: 95-101.

Schroeder, H.A and M. Mitchener. 1971. "Toxic effects of trace elements on the reproduction of mice and rats." Arch. Environ. Health. 23: 102-106.

Schroeder, H.A and M. Mitchener. 1975. "Life-term studies in rats: effects of aluminum, barium, beryllium, and tungsten." J. Nutr. 105: 421-427.

Sheldon, W.G. 1971. "The book of the american woodcock." The University of Massachusetts Press, Amberst, MA. 227 pp.

Skorupa, J.P. and R.L. Hothem. 1985. "Consumption of commercially-grown grapes by American robins: a field evaluation of laboratory estimates." J. Field Ornithol. 56(4); 369-378.

Skoryna, S.C. 1981. "Effects of oral supplementation with stable strontium." Can. Med. Assoc. J. 125: 703-712.

Sleight, S.D. and O.A. Atallah. 1968. "Reproduction in the guinea pig as affected by chronic administration of potassium nitrate and potassium nitrite." Toxicol. Appl. Pharmacol. 12: 179-185.

Smith W.P. 1991. "Odocoileus virginianus." Mammalian Species. 388: 1-13.

Spann, J.W., G.H. Heinz, and C.S. Hulse. 1986. "Reproduction and health in mallards fed endrin." Environ. Toxicol. Chem. 5: 755-759. 
Stickel, L.F., W.H. Stickel, R.A. Dyrland, and D.L. Hughes. 1983. "Oxychlordane, HCS3260, and nonachlor in birds: lethal residues and loss rates." J. Toxicol. Environ. Health. 12: 611-622.

Storm, G.L., R.D. Andrews, R. L. Phillips, R.A. Bishop, D.B. Siniff, and J.R. Tester. 1976. "Morphology, reproduction, dispersal, and mortality of midwestern red fox populations." Wildl. Monogr.

Suter, G.W., II. 1993. "Ecological risk assessment." Lewis Publ. Co., Boca Raton, Fl. 538 pp.

Tewe, O.O. and J.H. Maner. 1981. "Long-term and carry-over effect of dietary inorganic cyanide $(\mathrm{KCN})$ in the life cycle performance and metabolism of rats." Toxicol. Appl. Pharmacol. 58: 1-7.

Travis, C.C. and A.D. Arms. 1988. "Bioconcentration of organics in beef, milk, and vegetation." Environ. Sci. Technol. 22: 271-274.

Treon, J.F. and F.P. Cleveland. 1955. "Toxicity of certain chlorinated hydrocarbon insecticides for laboratory animals, with special reference to aldrin and dieldrin." Ag. Food Chem. 3: 402-408.

USAF (U.S. Air Force Systems Command). 1989. "The installation restoration program toxicology guide." Harry G. Armstrong Aerospace Medical Research Laboratory, WrightPatterson Air Force Base, OH.

U.S. Fish and Wildlife Service. 1964. "Pesticide-wildlife studies, 1963: a review of Fish and Wildlife Service investigations during the calendar year." FWS Circular 199.

U.S. Fish and Wildlife Service. 1969. "Bureau of sport fisheries and wildlife." Publication 74 , pp. 56-57.

Van Velsen, F.L., L.H.J.C. Danse, F.X.R. Van Leeuwen, J.A.M.A. Dormans, and M.J. Van Logten. 1986. "The subchronic oral toxicity of the beta-isomer of hexachlorocyclohexane in rats." Fund. Appl. Toxicol. 6: 697-712.

Verschuuren, H.G., R. Kroes, E.M. Den Tonkelaar, J.M. Berkvens, P.W. Helleman, A.G. Rauws, P.L. Schuller, and G.J. Van Esch. 1976. "Toxicity of methyl mercury chloride in rats. II. Reproduction study." Toxicol. 6: 97-106.

Villeneuve, D.C., D.L. Grant, K. Khera, D.J. Klegg, H. Baer, and W.E.J. Phillips, 1971. "The fetotoxicity of a polychlorinated biphenyl mixture (Aroclor 1254) in the rabbit and in the rat." Environ. Physiol. 1: 67-71.

Vogtsberger, L.M. and G.W. Barrett. 1973. "Bioenergetics of captive red foxes." J. Wildl. Manage. 37(4): 495-500. 
Vos, J.G., H.L. Van Der Maas, A.Musch, and E. Ram. 1971. "Toxicity of hexachlorobenzene in Japanese quail with special reference to porphyria, liver damage, reproduction, and tissue residues." Toxicol. Appl. Pharmacol. 18: 944-957.

Wakeley, J.S. 1978. "Activity budgets, energy expenditures, and energy intakes of nesting Ferruginous hawks." The Auk. 95: 667-676.

Whitaker, J.O. 1980. "The Audubon Society field guide to north American mammals." Alfred A. Knopf, New York, 745 pp.

White, D.H. and M.P. Dieter. 1978a. "Effects of dietary vanadium in mallard ducks." J. Toxicol. Environ. Health. 4: 43-50.

White, D.H. and M.T. Finley. 1978b. "Uptake and retention of dietary cadmium in mallard ducks". Environ. Res. 17: 53-59.

WHO (World Health Organization). 1984. "Chlordane." Environ. Health Criter. 34.82 pp.

Wobeser, G., N.O. Nielson, and B. Schiefer. 1976. "Mercury and mink II. Experimental methyl mercury intoxication." Can. J. Comp. Med. 34-45.

Woolson, E.A. (Ed.). 1975. "Arsenical pesticides." Am. Chem. Soc. Symp. Ser. 7. 176 pp. 
APPENDIX A

Descriptions of Studies Used to Calculate Benchmarks 
THIS PAGE INTENTIONALLY LEFT BLANK 


\title{
APPENDIX A. Descriptions of Studies Used to Calculate Benchmarks
}

\author{
Compound: $\quad$ Acetone \\ Form: not applicable \\ Reference: $\quad$ EPA $1986 c$ \\ Test Species: Rat
}

Body weight: $0.35 \mathrm{~kg}$ (EPA 1988a)

Food Consumption: $0.028 \mathrm{~kg} / \mathrm{d}$ (calculated using allometric equation from EPA 1988a)

Study Duration: 90 days $(<1 \mathrm{yr}$ and not during a critical lifestage $=$ subchronic $)$.

Endpoint:

Exposure Route:

Dosage:

Liver and kidney damage

oral intubation

three dose levels:

100,500 , and $2500 \mathrm{mg} / \mathrm{kg} / \mathrm{d} ; \mathrm{NOAEL}=100 \mathrm{mg} / \mathrm{kg} / \mathrm{d}$

Calculations:

not applicable

Comments: Significant tubular degeneration of the kidneys and increases in kidney weights were observed at the 500 and $2500 \mathrm{mg} / \mathrm{kg} / \mathrm{d}$ dose levels; liver weights were increased at the $2500 \mathrm{mg} / \mathrm{kg} / \mathrm{d}$ level. Because no significant differences were observed at the $100 \mathrm{mg} / \mathrm{kg} / \mathrm{d}$ dose level and the study considered exposure for 90 days and did not include critical lifestages (reproduction), this dose was considered to be a subchronic NOAEL. A chronic NOAEL was estimated by multiplying the subchronic NOAEL by a subchronic to chronic uncertainty factor of 0.1 .

Final NOAEL: $10 \mathrm{mg} / \mathrm{kg} / \mathrm{d}$

\section{Compound: $\quad$ Aldrin}

Form: not applicable

Reference: $\quad$ Treon and Cleveland 1955

Test Species: Rat $\quad$ Rat

Body weight: $0.35 \mathrm{~kg}$ (EPA 1988a)

Food Consumption: $0.028 \mathrm{~kg} / \mathrm{d}$ (calculated using allometric equation from EPA 1988a)

Exposure Duration: 3 generations ( $>1 \mathrm{yr}$ and during a critical lifestage $=$ chronic).

Endpoint: reproduction

Exposure Route:

Dosage: oral in diet three dose levels:

$$
2.5,12.5 \text {, and } 25.0 \mathrm{ppm} ; \mathrm{NOAEL}=2.5 \mathrm{ppm}
$$

\section{Calculations:}

$$
\left(\frac{2.5 \mathrm{mg} \text { Aldrin }}{\mathrm{kg} \text { food }} \times \frac{28 \mathrm{~g} \text { food }}{\text { day }} \times \frac{1 \mathrm{~kg}}{1000 \mathrm{~g}}\right) / 0.35 \mathrm{~kg} \mathrm{BW}=0.2 \mathrm{mg} / \mathrm{kg} / \mathrm{d}
$$

Comments: Because no significant differences were observed at the $2.5 \mathrm{ppm}$ dose 
level and the study considered exposure throughout 3 generations including critical lifestages (reproduction), this dose was considered to be a chronic NOAEL.

Final NOAEL: $0.2 \mathrm{mg} / \mathrm{kg} / \mathrm{d}$

Compound: Aluminum

Form: $\quad \mathrm{AlCl}_{3}$

Reference: $\quad$ Ondreicka et al. 1966

Test Species: Mouse

Body weight: $0.03 \mathrm{~kg}$ (EPA 1988a)

Exposure Duration: 3 generations $(>1 \mathrm{yr}$ and during a critical lifestage $=$ chronic).

Endpoint: reproduction

Exposure Route: oral in water

Dosage: one dose level:

Calculations: not applicable $19.3 \mathrm{mg} \mathrm{Al} / \mathrm{kg} / \mathrm{d}=$ LOAEL

Comments: While there were no effects on the number of litters or number of offspring per litter, growth of generations 2 and 3 was significantly reduced. Therefore, this dose was considered to be a chronic LOAEL. A chronic NOAEL was estimated by multiplying the chronic LOAEL by a LOAEL-NOAEL uncertainty factor of 0.1 .

Final NOAEL: $1.93 \mathrm{mg} / \mathrm{kg} / \mathrm{d}$

\section{Compound: $\quad$ Aluminum}

Form: $\quad \mathrm{Al}_{2}\left(\mathrm{SO}_{4}\right)_{3}$

Reference: $\quad$ Carriere et al. 1986

Test Species: $\quad$ Ringed Dove

Body weight: $0.155 \mathrm{~kg}$ (Terres 1980)

Food Consumption: $0.01727 \mathrm{~kg} / \mathrm{d}$ (calculated using allometric equation from Nagy 1987)

Exposure Duration: 4 months ( $>10 \mathrm{wk}$ and during a critical lifestage $=$ chronic).

Endpoint: reproduction

Exposure Route: oral in diet

Dosage: one dose level:

$1000 \mathrm{ppm} \mathrm{Al}\left(\right.$ as $\left.\mathrm{Al}_{2}\left(\mathrm{SO}_{4}\right)_{3}\right)=$ NOAEL

Calculations:

$$
\left(\frac{1000 \mathrm{mg} \mathrm{Al}}{\mathrm{kg} \text { food }} \times \frac{17.27 \mathrm{~g} \text { food }}{\text { day }} \times \frac{1 \mathrm{~kg}}{1000 \mathrm{~g}}\right) / 0.155 \mathrm{~kg} \mathrm{BW}=111.4 \mathrm{mg} / \mathrm{kg} / \mathrm{d}
$$

Comments: Because no significant differences were observed at the $1000 \mathrm{ppm}$ dose level and the study considered exposure over 4 months including critical lifestages (reproduction), this dose was considered to be a chronic NOAEL.

Final NOAEL: $111.4 \mathrm{mg} / \mathrm{kg} / \mathrm{d}$ 
$\begin{array}{cc}\text { Compound: } & \text { Antimony } \\ \text { Form: } & \text { Antimony Potassium Tartrate } \\ \text { Reference: } & \text { Schroeder et al. 1968b } \\ \text { Test Species: } & \text { Mouse }\end{array}$

Body weight: $0.03 \mathrm{~kg}$ (EPA 1988a)

Water Consumption: $0.0075 \mathrm{~L} / \mathrm{d}$ (calculated using allometric equation from EPA 1988a)

Exposure Duration: lifetime $(>1 \mathrm{yr}=$ chronic).

Endpoint: lifespan, longevity

Exposure Route: oral in water

Dosage: one dose level:

$5 \mathrm{ppm} \mathrm{Sb}=$ LOAEL

Calculations:

$$
\left(\frac{5 \mathrm{mg} \mathrm{Sb}}{L \text { water }} \times \frac{7.5 \mathrm{~mL} \text { water }}{\text { day }} \times \frac{1 \mathrm{~L}}{1000 \mathrm{~mL}}\right) / 0.03 \mathrm{~kg} \mathrm{BW}=1.25 \mathrm{mg} / \mathrm{kg} / \mathrm{d}
$$

Comments: Because median lifespan was reduced among female mice exposed to the $5 \mathrm{ppm}$ dose level and the study considered exposure throughout the entire lifespan, this dose was considered to be a chronic LOAEL. A chronic NOAEL was estimated by multiplying the chronic LOAEL by a LOAEL-NOAEL uncertainty factor of 0.1 .

Final NOAEL: $0.125 \mathrm{mg} / \mathrm{kg} / \mathrm{d}$

Compound: $\quad$ Aroclor 1016

Form: not applicable

Reference: Aulerich and Ringer 1980

Test Species: Mink

Body weight: $1.0 \mathrm{~kg}$ (EPA 1993)

food consumption: $0.137 \mathrm{~kg} / \mathrm{d}$ (Bleavins and Aulerich 1981)

Exposure Duration: 18 months $(>1 \mathrm{yr}$ and during a critical lifestage $=$ chronic).

Endpoint: reproduction

Exposure Route: oral in diet

Dosage: three dose levels:

Calculations:

$$
2,10 \text {, and } 25 \mathrm{ppm} ; 10 \mathrm{ppm}=\text { NOAEL }
$$

$$
\left(\frac{10 \mathrm{mg} \text { Aroclor } 1016}{\mathrm{~kg} \text { food }} \times \frac{137 \mathrm{~g} \mathrm{food}}{\text { day }} \times \frac{1 \mathrm{~kg}}{1000 \mathrm{~g}}\right) / 1 \mathrm{~kg} \mathrm{BW}=1.37 \mathrm{mg} / \mathrm{kg} / \mathrm{d}
$$

Comments: While kit mortality was greater for all dose levels, these differences were not significant. Because Aroclor 1016 at $25 \mathrm{ppm}$ in the diet reduced kit growth, and the study considered exposure over 18 months including critical lifestages (reproduction), the 
$10 \mathrm{ppm}$ dose was considered to be a chronic NOAEL.

Final NOAEL: $1.37 \mathrm{mg} / \mathrm{kg} / \mathrm{d}$

Compound: $\quad$ Aroclor 1242

Form: not applicable

Reference: Bleavins et al. 1980

Test Species: Mink

Body weight: $1.0 \mathrm{~kg}$ (EPA 1993)

food consumption: $0.137 \mathrm{~kg} / \mathrm{d}$ (Bleavins and Aulerich 1981)

Exposure Duration: 7 months (during a critical lifestage $=$ chronic).

Endpoint:

Exposure Route: oral in diet

Dosage:

Calculations:

four dose levels:

$5,10,20$, and $40 \mathrm{ppm} ; 5 \mathrm{ppm}=$ LOAEL

$$
\left[\frac{5 \mathrm{mg} \text { Aroclor } 1254}{\mathrm{~kg} \text { food }} \times \frac{137 \mathrm{~g} \text { food }}{\text { day }} \times \frac{1 \mathrm{~kg}}{1000 \mathrm{~g}}\right] / 1 \mathrm{~kg} \mathrm{BW}=0.685 \mathrm{mg} / \mathrm{kg} / \mathrm{d}
$$

Comments: Because all Aroclor 1242 dose levels produced total reproductive failure, and the study considered exposure over 7 months including critical lifestages (reproduction), the lowest dose was considered to be a chronic LOAEL. A chronic NOAEL was estimated by multiplying the chronic LOAEL by a LOAEL-NOAEL uncertainty factor of 0.1 .

Final NOAEL: $0.0685 \mathrm{mg} / \mathrm{kg} / \mathrm{d}$

Compound: $\quad$ Aroclor 1242

Form: not applicable

Reference: $\quad$ McLane and Hughes 1980

Test Species: $\quad$ Screech Owl

Body weight: $0.181 \mathrm{~kg}$ (Dunning 1984)

food consumption: 1300-1700 g/month/pair (Pattee et al. 1988)

Daily food consumption was estimated as follows:

median food consumption $/ \mathrm{month} /$ pair $=1500 \mathrm{~g}$;

1 month $=30 \mathrm{~d}$;

Males and females consume equal amounts of food $=750 \mathrm{~g} /$ month

$750 \mathrm{~g} /$ month $\div 30 \mathrm{~d}=25 \mathrm{~g} / \mathrm{d}$

Exposure Duration: 2 generations(during a critical lifestage $=$ chronic).

Endpoint:

Exposure Route:

reproduction

Dosage:

oral in diet

one dose level:

$3 \mathrm{ppm}=$ NOAEL 
Calculations:

$$
\left(\frac{3 \mathrm{mg} \text { Aroclor } 1242}{\mathrm{~kg} \text { food }} \times \frac{25 \mathrm{~g} \text { food }}{d a y} \times \frac{1 \mathrm{~kg}}{1000 \mathrm{~g}}\right) / 0.181 \mathrm{~kg} \mathrm{BW}=0.41 \mathrm{mg} / \mathrm{kg} / \mathrm{d}
$$

Comments: Fertility and hatching success was not significantly reduced by $3 \mathrm{ppm}$ Aroclor 1242 in the diet. Because the study considered exposure during reproduction, this dose was considered to be a chronic NOAEL.

Final NOAEL: $0.41 \mathrm{mg} / \mathrm{kg} / \mathrm{d}$

\section{Compound: $\quad$ Aroclor 1248}

Form: not applicable

Reference: Barsotti et al. 1976

Test Species: $\quad$ Rhesus Monkey

Body weight: $5.0 \mathrm{~kg}$ (from study)

food consumption: $0.2 \mathrm{~kg} / \mathrm{d}$ (EPA 1988a)

Exposure Duration: 14 months $(>1 \mathrm{yr}$ and during a critical lifestage $=$ chronic).

Endpoint: reproduction

Exposure Route: oral in diet

Dosage:

two dose levels:

2.5 and $5 \mathrm{ppm} ; 2.5 \mathrm{ppm}=\mathrm{LOAEL}$

\section{Calculations:}

$$
\left[\frac{2.5 \mathrm{mg} \text { Aroclor } 1248}{\mathrm{~kg} \text { food }} \times \frac{200 \mathrm{~g} \text { food }}{\text { day }} \times \frac{1 \mathrm{~kg}}{1000 \mathrm{~g}}\right] / 5 \mathrm{~kg} \mathrm{BW}=0.1 \mathrm{mg} / \mathrm{kg} / \mathrm{d}
$$

Comments: Pregnancy and live birth rates were reduced by both dose levels. Because the study considered exposure over 14 months including critical lifestages (reproduction), the $2.5 \mathrm{ppm}$ dose was considered to be a chronic LOAEL. A chronic NOAEL was estimated by multiplying the chronic LOAEL by a LOAEL-NOAEL uncertainty factor of 0.1 .

Final NOAEL: $0.01 \mathrm{mg} / \mathrm{kg} / \mathrm{d}$

\section{Compound:}

Form:

Reference:

Test Species:

Aroclor 1254

not applicable

Body weight: $1 \mathrm{~kg}$ (EPA 1993e)

Exposure Duration: 17 weeks $(>10 \mathrm{wks}$ and during a critical lifestage $=$ chronic).

Endpoint: reproduction

Exposure Route: weekly oral dose via gelatin capsule 
Dosage: two dose levels:

Calculations: $\quad 12.5 \mathrm{mg} / \mathrm{bird} /$ week $=1.8 \mathrm{mg} / \mathrm{kg} / \mathrm{d}$

12.5 and $50 \mathrm{mg} / \mathrm{bird} /$ week; LOAEL $=12.5 \mathrm{mg} / \mathrm{bird} /$ week

Comments: Significantly reduced egg hatchability was observed in both treatment groups. Therefore, because the study considered exposure throughout a critical lifestage (reproduction), the $12.5 \mathrm{mg} / \mathrm{bird} /$ week dose was considered to be a chronic LOAEL.

Final NOAEL: $0.18 \mathrm{mg} / \mathrm{kg} / \mathrm{d}$

Compound: $\quad$ Aroclor 1254

Form: not applicable

Reference: Linzey 1987

Test Species: White-footed mouse

Body weight: $0.02 \mathrm{~kg}$ (from study)

food consumption (from study): $0.135 \mathrm{~g}$ food $/ \mathrm{g} \mathrm{BW} / \mathrm{d}$ or $2.7 \mathrm{~g} / \mathrm{animal} / \mathrm{d}$

Exposure Duration: 18 months $(>1 \mathrm{yr}$ and during a critical lifestage $=$ chronic).

Endpoint: reproduction

Exposure Route: oral in diet

Dosage:

one dose level:

$10 \mathrm{ppm}=$ LOAEL

Calculations:

$\left(\frac{10 \mathrm{mg} \text { Aroclor } 1254}{\mathrm{~kg} \text { food }} \times \frac{2.7 \mathrm{~g} \text { food }}{\text { day }} \times \frac{1 \mathrm{~kg}}{1000 \mathrm{~g}}\right) / 0.02 \mathrm{~kg} \mathrm{BW}=1.35 \mathrm{mg} / \mathrm{kg} / \mathrm{d}$

Comments: Because Aroclor 1254 at $10 \mathrm{ppm}$ in the diet reduced the number of offspring per litter and the study considered exposure over 18 months including critical lifestages (reproduction), this dose was considered to be a chronic LOAEL. A chronic NOAEL was estimated by multiplying the chronic LOAEL by a LOAEL-NOAEL uncertainty factor of 0.1 .

Final NOAEL: $0.135 \mathrm{mg} / \mathrm{kg} / \mathrm{d}$

Compound: $\quad$ Aroclor 1254

Form: not applicable

Reference: Aulerich and Ringer 1977

Test Species: Mink

Body weight: $1.0 \mathrm{~kg}$ (EPA 1993e)

food consumption: $0.137 \mathrm{~kg} / \mathrm{d}$ (Bleavins and Aulerich 1981)

Exposure Duration: 4.5 month (during a critical lifestage $=$ chronic).

Endpoint:

reproduction

Exposure Route: oral in diet

Dosage:

three dose levels:

1,5 , and $15 \mathrm{ppm} ; \mathrm{NOAEL}=1 \mathrm{ppm}$. 
Calculations:

$$
\left(\frac{1 \mathrm{mg} \text { Aroclor } 1254}{\mathrm{~kg} \text { food }} \times \frac{137 \mathrm{~g} \text { food }}{\text { day }} \times \frac{1 \mathrm{~kg}}{1000 \mathrm{~g}}\right) / 1 \mathrm{~kg} \mathrm{BW}=0.137 \mathrm{mg} / \mathrm{kg} / \mathrm{d}
$$

Comments: Because Aroclor 1254 at 5 and $15 \mathrm{ppm}$ in the diet reduced the number of offspring born alive and the study considered exposure over 4.5 months days including critical lifestages (reproduction), the $1 \mathrm{ppm}$ dose was considered to be a chronic NOAEL.

Final NOAEL: $0.137 \mathrm{mg} / \mathrm{kg} / \mathrm{d}$

\section{Compound: $\quad$ Arsenic}

Form: $\quad$ Arsenite $\left(\mathrm{As}^{+3}\right)$

Reference: $\quad$ Schroeder and Mitchner 1971

Test Species: Mouse

Body weight: $0.03 \mathrm{~kg}$ (EPA 1988a)

Water Consumption: $0.0075 \mathrm{~L} / \mathrm{d}$

Food Consumption: $0.0055 \mathrm{~kg} / \mathrm{d}$

(calculated using allometric equation from EPA 1988a)

Exposure Duration: 3 generations $(>1 \mathrm{yr}$ and during critical lifestage $=$ chronic)

Endpoint:

reproduction

Exposure Route: oral in water (+ incidental in food; As species not stated,

Dosage: assumed to be $\mathrm{As}^{+3}$ )

one dose level:

Calculations:

$$
5 \mathrm{mg} \mathrm{As} / \mathrm{L} \text { (in water) }+0.06 \mathrm{mg} / \mathrm{kg} \mathrm{As} \text { (in food) }=\text { LOAEL }
$$

$$
\begin{aligned}
& \left(\frac{5 \mathrm{mg} \mathrm{As^{+3 }}}{L \text { water }} \times \frac{7.5 \mathrm{~mL} \text { water }}{\text { day }} \times \frac{1 \mathrm{~L}}{1000 \mathrm{~mL}}\right) / 0.03 \mathrm{~kg} \mathrm{BW}=1.25 \mathrm{mg} / \mathrm{kg} / \mathrm{d} \\
& \left(\frac{0.06 \mathrm{mg} \mathrm{As} \text { t3 }^{3}}{\mathrm{~kg} \text { food }} \times \frac{5.5 g \text { food }}{\text { day }} \times \frac{1 \mathrm{~kg}}{1000 \mathrm{~g}}\right) / 0.03 \mathrm{~kg} \mathrm{BW}=0.011 \mathrm{mg} / \mathrm{kg} / \mathrm{d} \\
& \text { Total Exposure }=1.25 \mathrm{mg} / \mathrm{kg} / \mathrm{d}+0.011 \mathrm{mg} / \mathrm{kg} / \mathrm{d}=1.261 \mathrm{mg} / \mathrm{kg} / \mathrm{d}
\end{aligned}
$$

Comments: Because mice exposed to $\mathrm{As}^{+3}$ displayed declining litter sizes with each successive generation and the study considered exposure over 3 generations, this dose was considered to be a chronic LOAEL. A chronic NOAEL was estimated by multiplying the chronic LOAEL by a LOAEL-NOAEL uncertainty factor of 0.1 .

Final NOAEL: $0.126 \mathrm{mg} / \mathrm{kg} / \mathrm{d}$ 
Compound: Arsenic

Form: Paris Green; Copper Acetoarsenite (44.34\% $\mathrm{As}^{+3}$ )

Reference: USFWS 1969

Test Species: $\quad$ Brown-headed Cowbird (Males only)

Body weight: $0.049 \mathrm{~kg}$ (Dunning 1984)

Food Consumption: $0.01087 \mathrm{~kg} / \mathrm{d}$

(calculated using allometric equation from Nagy 1987)

Exposure Duration: 7 months ( $>10 \mathrm{wk}=$ chronic)

Endpoint: mortality

Exposure Route: oral in diet

Dosage:

Calculations:

four dose level:

$25,75,225$, and $675 \mathrm{ppm}$ Paris Green; NOAEL $=25 \mathrm{ppm}$ $\mathrm{mg} / \mathrm{kg} \mathrm{As}{ }^{+3}=0.4434 \times 25 \mathrm{mg} / \mathrm{kg}=11.09 \mathrm{mg} / \mathrm{kg}$

$\left(\frac{11.09 \mathrm{mg} \mathrm{As^{+3 }}}{\mathrm{kg} \text { food }} \times \frac{10.87 \mathrm{~g} \mathrm{food}}{\text { day }} \times \frac{1 \mathrm{~kg}}{1000 \mathrm{~g}}\right) / 0.049 \mathrm{~kg} \mathrm{BW}=2.46 \mathrm{mg} / \mathrm{kg} / \mathrm{d}$

Comments: Cowbirds in the 675 and $225 \mathrm{ppm}$ groups experienced $100 \%$ mortality.

Those in the 75 and $25 \mathrm{ppm}$ groups experienced $20 \%$ and $0 \%$ mortality, respectively.

Because the study considered exposure over 7 months, the $25 \mathrm{ppm}$ Paris green $(11.09 \mathrm{mg} / \mathrm{kg}$ $\mathrm{As}^{+3}$ ) dose was considered to be a chronic NOAEL.

Final NOAEL: $2.46 \mathrm{mg} / \mathrm{kg} / \mathrm{d}$

\section{Compound: $\quad$ Arsenic}

Form: $\quad$ Sodium Arsenite $\left(51.35 \% \mathrm{As}^{+3}\right.$ )

Reference: USFWS 1964

Test Species: Mallard Ducks

Body weight: $1 \mathrm{~kg}$ (Heinz et al. 1989)

Food Consumption: $0.100 \mathrm{~kg} / \mathrm{d}$ (Heinz et al. 1989)

Exposure Duration: $128 \mathrm{~d}$ ( $>10 \mathrm{wk}=$ chronic)

Endpoint: mortality

Exposure Route: oral in diet

Dosage:

\section{Calculations:}

four dose level:

$100,250,500$, and 1000 ppm Sodium Arsenite;

NOAEL $=100 \mathrm{ppm}$

$\mathrm{mg} / \mathrm{kg} \mathrm{As}{ }^{+3}=0.5135 \times 100 \mathrm{mg} / \mathrm{kg}=51.35 \mathrm{mg} / \mathrm{kg}$

$$
\left(\frac{51.35 \mathrm{mg} \mathrm{As}}{\mathrm{kg} \text { food }} \times \frac{100 \mathrm{~g} \text { food }}{\text { day }} \times \frac{1 \mathrm{~kg}}{1000 \mathrm{~g}}\right) / 1 \mathrm{~kg} \mathrm{BW}=5.135 \mathrm{mg} / \mathrm{kg} / \mathrm{d}
$$


Comments: Mallards in the 1000,500 , and $250 \mathrm{ppm}$ groups experienced $92 \%, 60 \%$, and $12 \%$ mortality, respectively. Because those in the $100 \mathrm{ppm}$ group experienced $0 \%$ mortality, and the study considered exposure over 128 days, the $100 \mathrm{ppm}$ Sodium Arsenite ( $11.09 \mathrm{mg} / \mathrm{kg} \mathrm{As}^{+3}$ ) dose was considered to be a chronic NOAEL.

Final NOAEL: $5.135 \mathrm{mg} / \mathrm{kg} / \mathrm{d}$

$\begin{array}{cc}\text { Compound: } & \text { Barium } \\ \text { Form: } & \text { Barium Chloride } \\ \text { Reference: } & \text { Perry et al. } 1983 \\ \text { Test Species: } & \text { Rat }\end{array}$

Body weight: $0.435 \mathrm{~kg}$ (from study)

Water Consumption: $0.022 \mathrm{~L} / \mathrm{d}$ (from study)

Exposure Duration: 16 months $(>1 \mathrm{yr}=$ chronic $)$

Endpoint: growth, hypertension

Exposure Route: oral in water

Dosage:

three dose level:

1, 10, and 100, ppm Ba (as Barium Chloride);

NOAEL $=100 \mathrm{ppm}$

Calculations:

$$
\left(\frac{100 \mathrm{mg} \mathrm{Ba}}{L \text { water }} \times \frac{22 \mathrm{~mL} \text { water }}{\text { day }} \times \frac{1 \mathrm{~L}}{1000 \mathrm{~mL}}\right) / 0.435 \mathrm{~kg} \mathrm{BW}=5.06 \mathrm{mg} / \mathrm{kg} / \mathrm{d}
$$

Comments: While none of the three dose levels had any affect on food or water consumption or on growth, cardiovascular hypertension was observed among rats exposed to 10 or $100 \mathrm{ppm} \mathrm{Ba}$. Because the significance of hypertension in wild populations is unclear, the maximum dose that did not affect growth, food or water consumption (100 ppm) was considered to be a chronic NOAEL.

Final NOAEL: $5.06 \mathrm{mg} / \mathrm{kg} / \mathrm{d}$

\author{
Compound: Barium \\ Form: Barium Hydroxide \\ Reference: Johnson et al. 1960 \\ Test Species: 1-day old chicks \\ Body weight: $0.121 \mathrm{~kg}\left(\right.$ mean $_{\delta+\rho}$ at $14 \mathrm{~d}$; EPA 1988a) \\ Food Consumption: $0.0126 \mathrm{~kg} / \mathrm{d}$ (calculated using allometric equation from \\ EPA 1988a) \\ Exposure Duration: 4 wk $(<10 \mathrm{wk}=$ subchronic $)$ \\ Endpoint: mortality \\ Exposure Route: oral in diet
}


Dosage: $\quad$ eight dose level:

$250,500,1000,2000,4000,8000,16000$, and $32000 \mathrm{ppm}$

$\mathrm{Ba}$ (as Barium Hydroxide)

Calculations:

NOAEL $=2000 \mathrm{ppm}$

$$
\left(\frac{2000 \mathrm{mg} \mathrm{Ba}}{\mathrm{kg} \mathrm{food}} \times \frac{12.6 \mathrm{~g} \text { food }}{\text { day }} \times \frac{1 \mathrm{~kg}}{1000 \mathrm{~g}}\right) / 0.121 \mathrm{~kg} \mathrm{BW}=208.26 \mathrm{mg} / \mathrm{kg} / \mathrm{d}
$$

Comments: To estimate daily $\mathrm{Ba}$ intake throughout the 4 week study period, food consumption of 2-week-old chicks was calculated. While this value will over- and underestimate food consumption by younger and older chicks, it was assumed to approximate food consumption throughout the entire 4 week study. While Barium exposures up to 2000 ppm produced no mortality, chicks in the 4000 to $32000 \mathrm{ppm}$ groups experienced 5\% to $100 \%$ mortality. Because $2000 \mathrm{ppm}$ was the highest nonlethal dose, this dose was considered to be a subchronic NOAEL. A chronic NOAEL was estimated by multiplying the subchronic NOAEL by a subchronic to chronic uncertainty factor of 0.1 .

Final NOAEL: $20.826 \mathrm{mg} / \mathrm{kg} / \mathrm{d}$

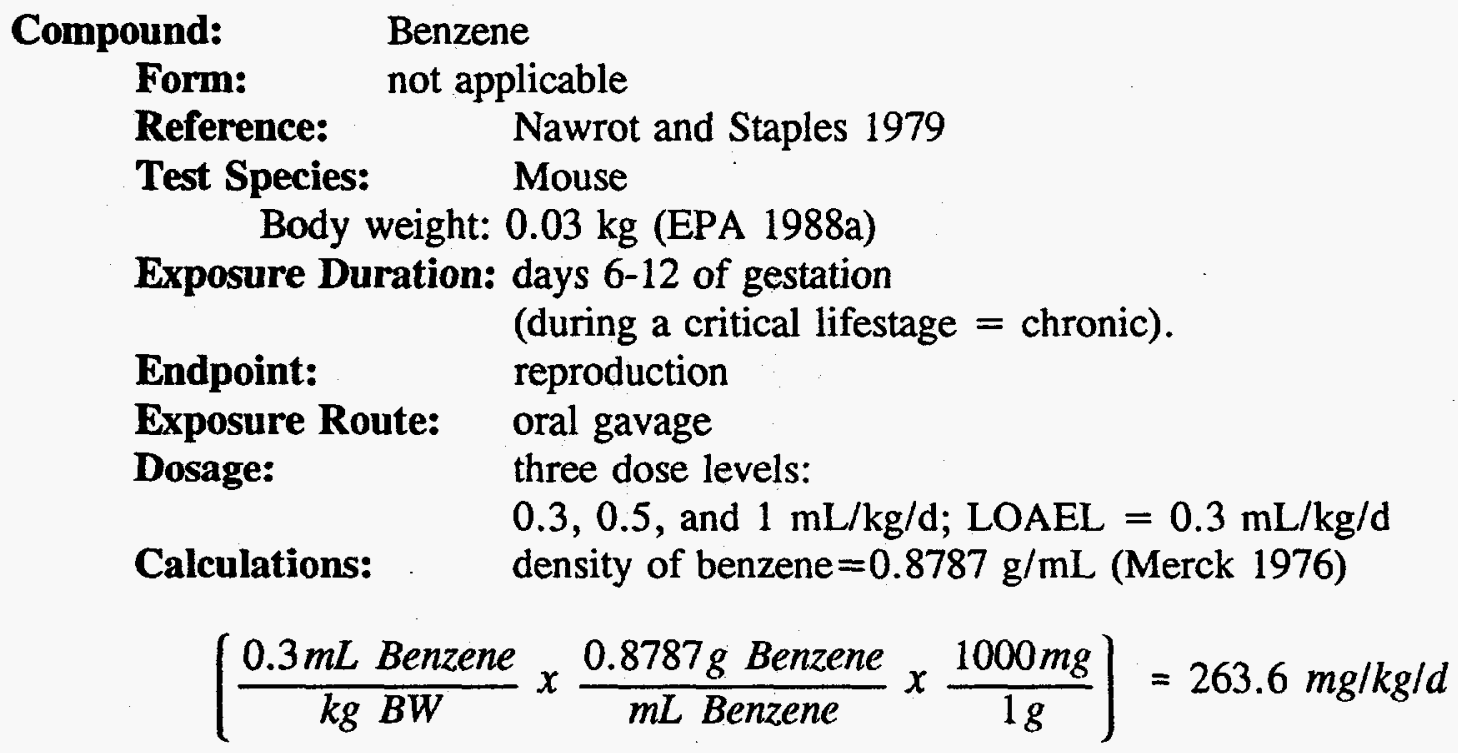

Comments: Benzene exposure of 0.5 and $1.0 \mathrm{~mL} / \mathrm{kg} / \mathrm{d}$ significantly increased maternal mortality and embryonic resorption. Fetal weights were significantly reduced by all three dose levels. While the benzene exposures evaluated in this study were of a short duration, they occurred during a critical lifestage. Therefore, the $0.3 \mathrm{~mL} / \mathrm{kg} / \mathrm{d}$ dose was considered to be a chronic LOAEL. A chronic NOAEL was estimated by multiplying the chronic LOAEL by a LOAEL-NOAEL uncertainty factor of 0.1 .

Final NOAEL: $26.36 \mathrm{mg} / \mathrm{kg} / \mathrm{d}$ 
Compound: $\quad \beta$-Benzene Hexachloride $(\beta$-BHC)

Form: not applicable

Reference: Van Velsen et al. 1986

Test Species: Rat

Body weight: $0.35 \mathrm{~kg}$ (EPA 1988a)

Food Consumption: $0.028 \mathrm{~kg} / \mathrm{d}$ (calculated using allometric equation from EPA 1988a)

Exposure Duration: 13 weeks

( $<1 \mathrm{yr}$ and not during a critical lifestage $=$ subchronic $)$.

Endpoint:

Exposure Route: growth, blood chemistry, organ histology

Dosage: oral in diet

four dose levels:

$2,10,50$, and $250 \mathrm{ppm} ; \mathrm{NOAEL}=50 \mathrm{ppm}$

Calculations:

$$
\left(\frac{50 \mathrm{mg} \beta-B H C}{\mathrm{~kg} \text { food }} \times \frac{28 \mathrm{~g} \text { food }}{d a y} \times \frac{1 \mathrm{~kg}}{1000 \mathrm{~g}}\right) / 0.35 \mathrm{~kg} \mathrm{BW}=4 \mathrm{mg} / \mathrm{kg} / \mathrm{d}
$$

Comments: Consumption of $250 \mathrm{ppm} \beta$-BHC in the diet caused gonadal atrophy in both male and female rats. Because no significant effects were observed in groups consuming $50 \mathrm{ppm} \beta-\mathrm{BHC}$ or less, this dose was considered to be a subchronic NOAEL. A chronic NOAEL was estimated by multiplying the subchronic NOAEL by a subchronicchronic uncertainty factor of 0.1 .

Final NOAEL: $0.4 \mathrm{mg} / \mathrm{kg} / \mathrm{d}$

Compound: $\quad$ Benzene Hexachloride (BHC mixed isomers)

Form: not applicable

Reference: Bleavins et al. 1984

Test Species: Mink

Body weight: $1.0 \mathrm{~kg}$ (EPA 1993e)

food consumption: $0.137 \mathrm{~kg} / \mathrm{d}$ (Bleavins and Aulerich 1981)

Exposure Duration: $331 \mathrm{~d}$ (during a critical lifestage $=$ chronic).

Endpoint: reproduction

Exposure Route: oral in diet

Dosage:

three dose levels:

1,5 , and $25 \mathrm{ppm} ; 1 \mathrm{ppm}=$ LOAEL

Calculations:

$$
\left(\frac{1 \mathrm{mg} \mathrm{BHC}}{\mathrm{kg} \text { food }} \times \frac{137 \mathrm{~g} \text { food }}{\text { day }} \times \frac{1 \mathrm{~kg}}{1000 \mathrm{~g}}\right) / 1 \mathrm{~kg} \mathrm{BW}=0.137 \mathrm{mg} / \mathrm{kg} / \mathrm{d}
$$




\section{A-14}

Comments: All dose levels produced increased kit mortality and decreased kit body weight. Because the study considered exposure over 331 days including critical lifestages (reproduction), this dose was considered to be a chronic LOAEL. A chronic NOAEL was estimated by multiplying the chronic LOAEL by a LOAEL-NOAEL uncertainty factor of 0.1 .

Final NOAEL: $0.0137 \mathrm{mg} / \mathrm{kg} / \mathrm{d}$

Compound: Benzene Hexachloride (BHC mixed isomers)

Form: not applicable

Reference: Grant et al. 1977

Test Species: $\quad$ Rat

Body weight: $0.35 \mathrm{~kg}$ (EPA 1988a)

Food Consumption: $0.028 \mathrm{~kg} / \mathrm{d}$ (calculated using allometric equation from EPA 1988a)

Exposure Duration: 4 generations ( $>1 \mathrm{yr}$ and during a critical lifestage $=$ chronic).

Endpoint: reproduction

Exposure Route: oral in diet

Dosage:

seven dose levels:

$10,20,40,80,160,320$, and $640 \mathrm{ppm} ;$ NOAEL $=20 \mathrm{ppm}$

\section{Calculations:}

$$
\left(\frac{20 \mathrm{mg} \mathrm{BHC}}{\mathrm{kg} \mathrm{food}} \times \frac{28 \mathrm{~g} \text { food }}{\text { day }} \times \frac{1 \mathrm{~kg}}{1000 \mathrm{~g}}\right) / 0.35 \mathrm{~kg} \mathrm{BW}=1.6 \mathrm{mg} / \mathrm{kg} / \mathrm{d}
$$

Comments: Consumption of $320 \mathrm{ppm}$ and $640 \mathrm{ppm}$ BHC in the diet increased maternal mortality, 80 - 640 ppm BHC reduced litter sizes, and $40-320$ ppm BHC reduced birthweights. Because no significant effects were observed in groups consuming 10 or 20 ppm BHC in their diet and the study considered exposure throughout four generations including critical lifestages (reproduction), the $20 \mathrm{ppm}$ dose was considered to be a chronic NOAEL.

Final NOAEL: $1.6 \mathrm{mg} / \mathrm{kg} / \mathrm{d}$

Compound: $\quad$ Benzene Hexachloride (BHC mixed isomers)

Form: not applicable

Reference: $\quad$ Vos et al. 1971

Test Species: Japanese Quail

Body weight: $0.150 \mathrm{~kg}$ (from study)

Food Consumption: $0.0169 \mathrm{~kg} / \mathrm{d}$ (calculated using allometric equation from Nagy 1987)

Exposure Duration: $90 \mathrm{~d}$ (during a critical lifestage $=$ chronic).

Endpoint:

reproduction 
Exposure Route: oral in diet

Dosage:

seven dose levels:

Calculations:

$$
1,5,20 \text {, and } 80 \mathrm{ppm} ; \text { NOAEL }=5 \mathrm{ppm}
$$

$$
\left(\frac{5 \mathrm{mg} \mathrm{BHC}}{\mathrm{kg} \mathrm{food}} \times \frac{16.9 \mathrm{~g} \mathrm{food}}{\text { day }} \times \frac{1 \mathrm{~kg}}{1000 \mathrm{~g}}\right) / 0.15 \mathrm{~kg} \mathrm{BW}=0.563 \mathrm{mg} / \mathrm{kg} / \mathrm{d}
$$

Comments: Consumption of $20 \mathrm{ppm}$ and $80 \mathrm{ppm}$ BHC in the diet reduced egg hatchability and egg volume. Because no significant effects were observed in groups consuming 1 or $5 \mathrm{ppm} \mathrm{BHC}$ in their diet and the study considered exposure throughout a critical lifestage (reproduction), the $5 \mathrm{ppm}$ dose was considered to be a chronic NOAEL.

Final NOAEL: $0.563 \mathrm{mg} / \mathrm{kg} / \mathrm{d}$

\author{
Compound: $\quad$ Benzo(a)pyrene (BaP) \\ Form: not applicable \\ Reference: $\quad$ Mackenzie and Angevine 1981 \\ Test Species: Mouse
}

Body weight: $0.03 \mathrm{~kg}$ (EPA 1988a)

Exposure Duration: days 7-16 of gestation (during a critical lifestage $=$ chronic).

Endpoint: reproduction

Exposure Route: oral intubation

Dosage:

Calculations: three dose levels:

10,40 , and $160 \mathrm{mg} / \mathrm{kg} / \mathrm{d} ;$ LOAEL $=10 \mathrm{mg} / \mathrm{kg} / \mathrm{d}$

Comments: BaP exposure $160 \mathrm{mg} / \mathrm{kg} / \mathrm{d}$ significantly reduced pregnancy rates and percentage of viable litters. Pup weights were significantly reduced by all three dose levels. Total sterility was observed in $97 \%$ of offspring in the 40 and $160 \mathrm{mg} / \mathrm{kg} / \mathrm{d}$ groups and fertility was impaired among offspring in the $10 \mathrm{mg} / \mathrm{kg} / \mathrm{d}$ group. While the BaP exposures evaluated in this study were of a short duration, they occurred during a critical lifestage. Therefore, the $10 \mathrm{mg} / \mathrm{kg} / \mathrm{d}$ dose was considered to be a chronic LOAEL. A chronic NOAEL was estimated by multiplying the chronic LOAEL by a LOAEL-NOAEL uncertainty factor of 0.1 .

Final NOAEL: $1 \mathrm{mg} / \mathrm{kg} / \mathrm{d}$
Compound:
Beryllium
Form: Beryllium Sulfate
Reference: Schroeder and Mitchner 1975 
Test Species: Rat

Body weight: $0.35 \mathrm{~kg}$ (EPA 1988a)

Water Consumption: $0.046 \mathrm{~L} / \mathrm{d}$ (calculated using allometric equation from EPA 1988a)

Exposure Duration: lifetime $(>1 \mathrm{yr}=$ chronic)

Endpoint:

longevity, weight loss

Exposure Route: oral in water

Dosage:

one dose level:

$5 \mathrm{ppm} \mathrm{Be}=$ NOAEL

Calculations:

$$
\left(\frac{5 \mathrm{mg} \mathrm{Be}}{L \text { water }} \times \frac{46 \mathrm{~mL} \text { water }}{\text { day }} \times \frac{1 \mathrm{~L}}{1000 \mathrm{~mL}}\right) / 0.35 \mathrm{~kg} \mathrm{BW}=0.66 \mathrm{mg} / \mathrm{kg} / \mathrm{d}
$$

Comments: While exposure to $5 \mathrm{ppm}$ Be in water did not reduce longevity, weight loss by males was observed in months $2-6$. Because the weight loss was not considered to be an adverse effect, the $5 \mathrm{ppm}$ dose level was considered to be a chronic NOAEL.

Final NOAEL: $0.66 \mathrm{mg} / \mathrm{kg} / \mathrm{d}$

Compound: $\quad$ Bis(2-ethylhexyl)Phthalate (BEHP)

Form: not applicable

Reference: Lamb et al. 1987

Test Species: Mouse

Body weight: $0.03 \mathrm{~kg}$ (EPA 1988a)

Food Consumption: $0.0055 \mathrm{~kg} / \mathrm{d}$

(calculated using allometric equation from EPA 1988a)

Exposure Duration: $105 \mathrm{~d}$ (during critical lifestage = chronic).

Endpoint: reproduction

Exposure Route: oral in diet

Dosage:

three dose levels:

$0.01 \%, 0.1 \%$ and $0.3 \%$ of diet;

NOAEL $=0.01 \%=100 \mathrm{mg} / \mathrm{kg}$

Calculations:

$$
\left(\frac{100 \mathrm{mg} \mathrm{BEHP}}{\mathrm{kg} \mathrm{food}} \times \frac{5.5 \mathrm{~g} \mathrm{food}}{\text { day }} \times \frac{1 \mathrm{~kg}}{1000 \mathrm{~g}}\right) / 0.03 \mathrm{~kg} \mathrm{BW}=18.33 \mathrm{mg} / \mathrm{kg} / \mathrm{d}
$$

Comments: While significant reproductive effects were observed among mice on diets containing $0.1 \%$ and $0.3 \%$ Bis(2-ethylhexyl)Phthalate, no adverse effects were observed among the $0.01 \%$ dose group. Because the study considered exposure during critical lifestage, the $0.01 \%$ dose was considered to be a chronic NOAEL.

Final NOAEL: $18.33 \mathrm{mg} / \mathrm{kg} / \mathrm{d}$ 
Compound: $\quad$ Bis(2-ethylhexyl)Phthalate (BEHP)

Form: not applicable

Reference: $\quad$ Peakall 1974

Test Species: $\quad$ Ringed Dove

Body weight: $0.155 \mathrm{~kg}$ (Terres 1980)

Food Consumption: $0.01727 \mathrm{~kg} / \mathrm{d}$ (calculated using allometric equation from ... Nagy 1987)

Exposure Duration: 4 weeks (during critical lifestage $=$ chronic).

Endpoint:

Exposure Route:

Dosage: reproduction

oral in diet

one dose level:

$10 \mathrm{ppm}=$ NOAEL

\section{Calculations:}

$$
\left[\frac{10 \mathrm{mg} \text { BEHP }}{\mathrm{kg} \text { food }} \times \frac{17.27 \mathrm{~g} \text { food }}{\text { day }} \times \frac{1 \mathrm{~kg}}{1000 \mathrm{~g}}\right] / 0.155 \mathrm{~kg} \mathrm{BW}=1.11 \mathrm{mg} / \mathrm{kg} / \mathrm{d}
$$

Comments: No significant reproductive effects were observed among doves on diets containing $10 \mathrm{ppm}$ Bis(2-ethylhexyl)Phthalate, and the study considered exposure over 4 weeks and during a critical lifestage, the $10 \mathrm{ppm}$ dose was considered to be a chronic NOAEL. .

Final NOAEL: $1.11 \mathrm{mg} / \mathrm{kg} / \mathrm{d}$

Compound: Cadmium

Form: soluble salt

Reference: $\quad$ Schroeder and Mitchner 1971

Test Species: $\quad$ Mouse

Body weight: $0.03 \mathrm{~kg}$ (EPA 1988a)

Water Consumption: $0.0075 \mathrm{~L} / \mathrm{d}$

Food Consumption: $0.0055 \mathrm{~kg} / \mathrm{d}$

(calculated using allometric equation from EPA 1988a)

Exposure Duration: 2 generations $(>1 \mathrm{yr}$ and during a critical lifestage $=$ chronic).

Endpoint: reproduction

Exposure Route: oral in water (+incidental in food)

Dosage:

one dose level:

$10 \mathrm{ppm} \mathrm{Cd}$ (in water) $+0.1 \mathrm{ppm} \mathrm{Cd}$ (in food) $=$ LOAEL 
Calculations:

$$
\begin{gathered}
\left(\frac{10 \mathrm{mg} C d}{L \text { water }} \times \frac{7.5 \mathrm{~mL} \text { water }}{\text { day }} \times \frac{1 \mathrm{~L}}{1000 \mathrm{~mL}}\right) / 0.03 \mathrm{~kg} \mathrm{BW}=2.5 \mathrm{mg} / \mathrm{kg} / \mathrm{d} \\
\left(\frac{0.1 \mathrm{mg} C d}{\mathrm{~kg} \text { food }} \times \frac{5.5 \mathrm{~g} \text { food }}{\text { day }} \times \frac{1 \mathrm{~kg}}{1000 \mathrm{~g}}\right) / 0.03 \mathrm{~kg} \mathrm{BW}=0.018 \mathrm{mg} / \mathrm{kg} / \mathrm{d} \\
\text { Total Exposure }=2.5 \mathrm{mg} / \mathrm{kg} / \mathrm{d}+0.018 \mathrm{mg} / \mathrm{kg} / \mathrm{d}=2.518 \mathrm{mg} / \mathrm{kg} / \mathrm{d}
\end{gathered}
$$

Comments: Because mice exposed to Cd displayed reduced reproductive success (the strain did not survive to the third generation) and congenital deformities, this dose was considered to be a chronic LOAEL. A chronic NOAEL was estimated by multiplying the chronic LOAEL by a LOAEL-NOAEL uncertainty factor of 0.1 .

Final NOAEL: $0.1913 \mathrm{mg} / \mathrm{kg} / \mathrm{d}$
Compound: $\quad$ Cadmium
Form: $\quad$ Cadmium Chloride
Reference: White and Finley 1978
Test Species: Mallard Ducks
Body weight: $1.153 \mathrm{~kg}$ (from study)
Food Consumption: $0.110 \mathrm{~kg} / \mathrm{d}$ (from study)
Endpoint: reproduction
Exposure Route: oral in diet
Dosage: three dose level:

$$
1.6,15.2 \text {, and } 210 \mathrm{ppm} \mathrm{Cd}
$$$$
\text { NOAEL }=15.2 \mathrm{ppm}
$$

Exposure Duration: $90 \mathrm{~d}$ ( $>10 \mathrm{wk}$ and during a critical lifestage $=$ chronic)

\section{Calculations:}$$
\left(\frac{15.2 \mathrm{mgCd}}{\mathrm{kg} \mathrm{food}} \times \frac{110 \mathrm{~g} \text { food }}{d a y} \times \frac{1 \mathrm{~kg}}{1000 \mathrm{~g}}\right) / 1.153 \mathrm{~kg} \mathrm{BW}=1.45 \mathrm{mg} / \mathrm{kg} / \mathrm{d}
$$

Comments: Mallards in the $210 \mathrm{ppm}$ group produced significantly fewer eggs than those in the other groups. Because the study considered exposure over 90 days, the 15.2 ppm Cd dose was considered to be a chronic NOAEL.

Final NOAEL: $1.45 \mathrm{mg} / \mathrm{kg} / \mathrm{d}$

Compound:

Form:

Reference:
Carbon Tetrachloride

not applicable

Alumot at al. 1976a 
Test Species: Rat

Body weight: $0.35 \mathrm{~kg}$ (EPA 1988a)

Food Consumption: $0.028 \mathrm{~kg} / \mathrm{d}$ (calculated using allometric equation from EPA 1988a)

Exposure Duration: 2 yr $(>1$ yr and during a critical lifestage $=$ chronic).

Endpoint: reproduction

Exposure Route: oral in diet

Dosage: two dose levels:

80 and $200 \mathrm{ppm}$;

No effects observed at either dose level.

Calculations:

$$
\left(\frac{200 \mathrm{mg} \mathrm{CCl}}{\mathrm{kg} \text { food }} \times \frac{28 \mathrm{~g} \text { food }}{\text { day }} \times \frac{1 \mathrm{~kg}}{1000 \mathrm{~g}}\right) / 0.35 \mathrm{~kg} \mathrm{BW}=16 \mathrm{mg} / \mathrm{kg} / \mathrm{d}
$$

Comments: Because no significant differences were observed at either dose level and the study considered exposure throughout 2 years including critical lifestages (reproduction), the maximum dose was considered to be a chronic NOAEL.

Final NOAEL: $16 \mathrm{mg} / \mathrm{kg} / \mathrm{d}$

Compound: $\quad$ Chlordane

Form: not applicable

Reference: WHO 1984 (secondary source; Primary citation: Keplinger, M.L., W.B. Deichman, and F. Sala. 1968. Effects of

Test Species: Mouse pesticides on reproduction in mice. Ind. Med. Surg. 37: 525.) Body weight: $0.03 \mathrm{~kg}$ (EPA 1988a)

Food Consumption: $0.0055 \mathrm{~kg} / \mathrm{d}$

(calculated using allometric equation from EPA 1988a)

Exposure Duration: 6 generations $(>1 \mathrm{yr}$ and during a critical lifestage $=$ chronic).

Endpoint: reproduction

Exposure Route: oral in diet

Dosage: three dose levels:

Calculations: 25,50 , and $100 \mathrm{mg} / \mathrm{kg} ; \mathrm{NOAEL}=25 \mathrm{mg} / \mathrm{kg}$

$$
\left(\frac{25 \mathrm{mg} \text { Chlordane }}{\mathrm{kg} \mathrm{food}} \times \frac{5.5 \mathrm{~g} \text { food }}{\text { day }} \times \frac{1 \mathrm{~kg}}{1000 \mathrm{~g}}\right) / 0.03 \mathrm{~kg} \mathrm{BW}=4.58 \mathrm{mg} / \mathrm{kg} / \mathrm{d}
$$

Comments: While significant effects were observed among mice on diets containing 50 and $100 \mathrm{mg} / \mathrm{kg}$ Chlordane (decreased viability and reduced abundance of offspring), no 
adverse effects were observed among the $25 \mathrm{mg} / \mathrm{kg}$ dose group. Because the study considered exposure over six generations and through reproduction, the $25 \mathrm{mg} / \mathrm{kg}$ dose was considered to be a chronic NOAEL.

Final NOAEL: $4.58 \mathrm{mg} / \mathrm{kg} / \mathrm{d}$

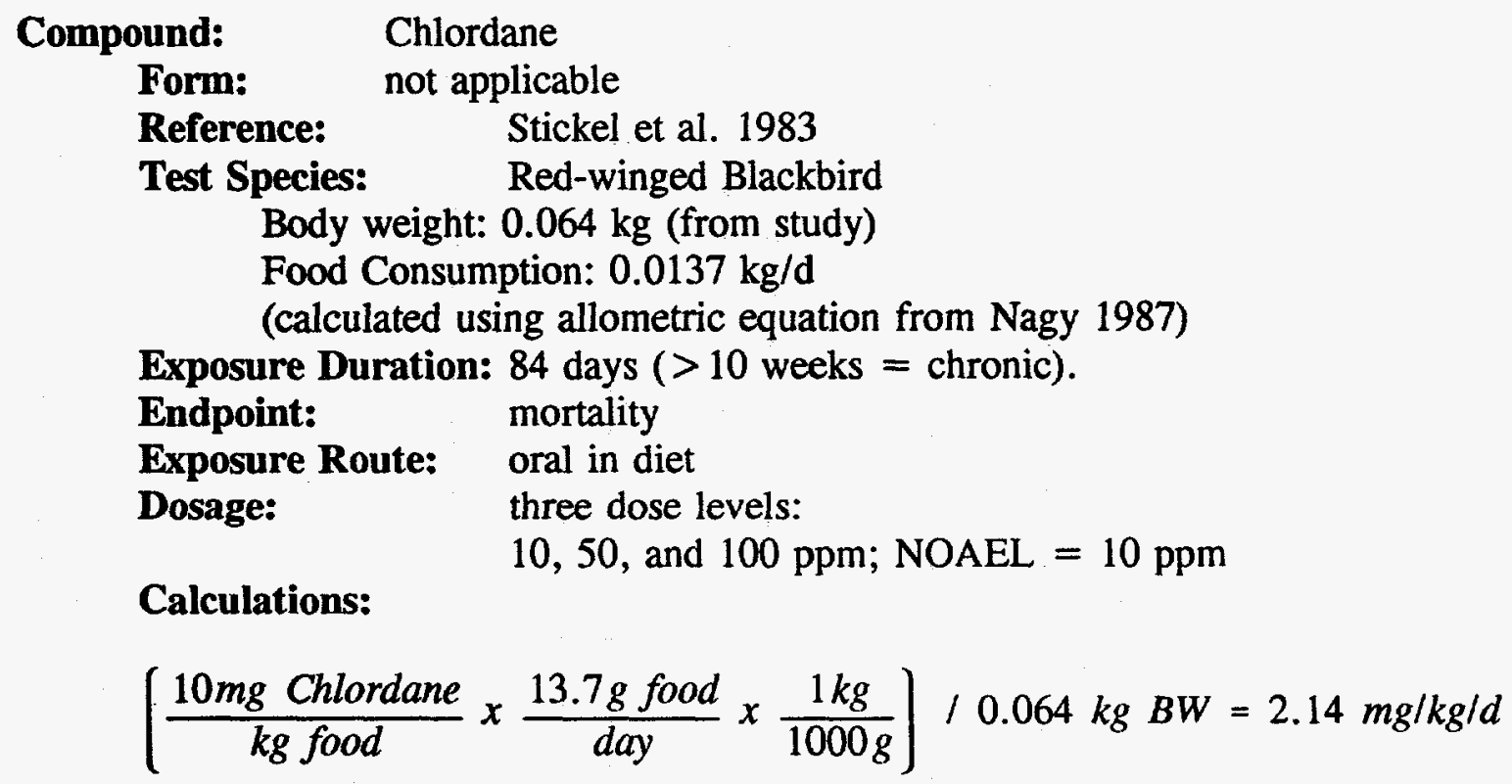

Comments: While $26 \%$ and $24 \%$ mortality was observed among birds on diets containing 50 and $100 \mathrm{mg} / \mathrm{kg}$ Chlordane, no adverse effects were observed among the 10 $\mathrm{mg} / \mathrm{kg}$ dose group. Because the study considered exposure over 84 days, the $10 \mathrm{mg} / \mathrm{kg}$ dose was considered to be a chronic NOAEL.

Final NOAEL: $2.14 \mathrm{mg} / \mathrm{kg} / \mathrm{d}$

$\begin{array}{lc}\text { Compound: } & \text { Chlordecone (Kepone) } \\ \text { Form: } & \text { not applicable } \\ \text { Reference: } & \text { Larson et al. } 1979 \\ \text { Test Species: } & \text { Rat }\end{array}$

Body weight: $0.35 \mathrm{~kg}$ (EPA 1988a)

Food Consumption: $0.028 \mathrm{~kg} / \mathrm{d}$ (calculated using allometric equation from EPA 1988a)

Exposure Duration: 2 yr $(>1$ yr and during a critical lifestage $=$ chronic).

Endpoint:

Exposure Route:

Dosage: mortality, growth, kidney damage

oral in diet

five dose levels:

$1,5,10,25$, and $80 \mathrm{ppm} ; \mathrm{NOAEL}=1 \mathrm{ppm}$ 


\title{
Calculations:
}

$$
\left(\frac{1 \mathrm{mg} \text { Chlordecone }}{\mathrm{kg} \text { food }} \times \frac{28 \mathrm{~g} \mathrm{food}}{\mathrm{day}} \times \frac{1 \mathrm{~kg}}{1000 \mathrm{~g}}\right) / 0.35 \mathrm{~kg} \mathrm{BW}=0.08 \mathrm{mg} / \mathrm{kg} / \mathrm{d}
$$

Comments: Chlordecone at 25 and $80 \mathrm{ppm}$ in the diet produced $100 \%$ mortality in 6 months. Growth was depressed by 10 and $25 \mathrm{ppm}$ and kidney damage was observed at doses as low as $5 \mathrm{ppm}$. Because the study considered exposure throughout 2 years, the $1 \mathrm{ppm}$ dose was considered to be a chronic NOAEL.

Final NOAEL: $0.08 \mathrm{mg} / \mathrm{kg} / \mathrm{d}$

\author{
Compound: Chloroform \\ Form: not applicable \\ Reference: $\quad$ Palmer et al. 1979 \\ Test Species: Rat
}

Body weight: $0.35 \mathrm{~kg}$ (EPA 1988a)

Exposure Duration: $13 \mathrm{wk}(<1 \mathrm{yr}$ and not during a critical lifestage $=$ subchronic).

Endpoint: liver, kidney, gonad condition

Exposure Route: oral intubation

Dosage:

Calculations: four dose levels:

$15,30,150$, and $410 \mathrm{mg} / \mathrm{kg} / \mathrm{d} ;$ NOAEL $=150 \mathrm{mg} / \mathrm{kg} / \mathrm{d}$

Comments: Gonadal atrophy was observed among male and female rats receiving $410 \mathrm{mg} / \mathrm{kg} / \mathrm{d}$; therefore $150 \mathrm{mg} / \mathrm{kg} / \mathrm{d}$ was considered to be a subchronic NOAEL. To estimate the chronic NOAEL, the subchronic NOAEL was multiplied by a subchronicchronic uncertainty factor of 0.1 .

Final NOAEL: $15 \mathrm{mg} / \mathrm{kg} / \mathrm{d}$

Compound: $\quad$ Chromium

$\begin{array}{lc}\text { Form: } & \mathrm{Cr}^{+3} \text { as } \mathrm{Cr}_{2} \mathrm{O}_{3} \quad(68.42 \% \mathrm{Cr}) \\ \text { Reference: } & \text { Ivankovic and Preussmann } 1975 \\ \text { Test Species: } & \text { Rat }\end{array}$

Body weight: $0.35 \mathrm{~kg}$ (EPA 1988a)

Food Consumption: $0.028 \mathrm{~kg} / \mathrm{d}$ (calculated using allometric equation from EPA 1988a)

Exposure Duration: $90 \mathrm{~d}$ and $2 \mathrm{yr}$

Endpoint:

Exposure Route:

Dosage: reproduction, longevity

oral in diet

three dose levels:

$\mathrm{Cr}_{2} \mathrm{O}_{3}$ as $1 \%, 2 \%$ or $5 \%$ of diet

No effects observed at any dose level 
Calculations:

$$
\left(\frac{50,000 \mathrm{mg} \mathrm{Cr} \mathrm{O}_{3}}{\mathrm{~kg} \text { food }} \times \frac{28 \mathrm{~g} \text { food }}{d a y} \times \frac{1 \mathrm{~kg}}{1000 \mathrm{~g}}\right) / 0.35 \mathrm{~kg} \mathrm{BW}=4000 \mathrm{mg} / \mathrm{kg} / \mathrm{d}
$$

$0.6842 \times 4000 \mathrm{mg} \mathrm{Cr} \mathrm{O}_{3} / \mathrm{kg} / \mathrm{d}$ or $2737 \mathrm{mg} \mathrm{Cr}{ }^{+3} / \mathrm{kg} / \mathrm{d}$.

Comments: Reproductive effects were evaluated among rats fed $2 \%$ or $5 \% \mathrm{Cr}_{2} \mathrm{O}_{3}$ for $90 \mathrm{~d}$; carcinogenicity and longevity were evaluated among rats fed $1 \%, 2 \%$ or $5 \% \mathrm{Cr}_{2} \mathrm{O}_{3}$ for 2 years. Because no significant differences were observed at any dose level in either study and both studies considered exposure throughout 2 years or a critical lifestage (reproduction), the maximum dose was considered to be a chronic NOAEL.

Final NOAEL: $2737 \mathrm{mg} / \mathrm{kg} / \mathrm{d}$

$\begin{array}{lcl}\text { Compound: } & \text { Chromium } \\ \text { Form: } & \mathrm{Cr}^{+6} \text { as } \mathrm{K}_{2} \mathrm{Cr}_{2} \mathrm{O}_{4} \\ \text { Reference: } & \text { MacKenzie et al. } & 1958 \\ \text { Test Species: } & \text { Rat }\end{array}$

Body weight: $0.35 \mathrm{~kg}$ (EPA 1988a)

Water Consumption: $0.046 \mathrm{~L} / \mathrm{d}$ (calculated using allometric equation from EPA 1988a)

Exposure Duration: 1 yr

Endpoint: body weight and food consumption

Exposure Route: oral in water

Dosage: six dose levels:

$0.45,2.2,4.5,7.7,11.2$, and $25 \mathrm{ppm} \mathrm{Cr}^{+6}$ in diet No effects observed at any dose level

Calculations:

$$
\left(\frac{25 \mathrm{mg} \mathrm{Cr^{+6 }}}{L \text { water }} \times \frac{0.046 L \text { water }}{\text { day }}\right] / 0.35 \mathrm{~kg} \mathrm{BW}=3.28 \mathrm{mg} / \mathrm{kg} / \mathrm{d}
$$

Comments: Because no significant differences were observed at any dose level studied and the study considered exposure over 1 year, the maximum dose was considered to be a chronic NOAEL.

Final NOAEL: $3.28 \mathrm{mg} / \mathrm{kg} / \mathrm{d}$

\author{
Compound: Chromium \\ Form: $\quad \mathrm{Cr}^{+3}$ as $\mathrm{CrK}\left(\mathrm{SO}_{4}\right)_{2}$ \\ Reference: Haseltine et al. , unpubl. data
}


Test Species: $\quad$ Black duck

Body weight: $1.25 \mathrm{~kg}$ (mean $_{\delta+q} ;$ Dunning 1984)

Food Consumption: Congeneric Mallard ducks, weighing $1 \mathrm{~kg}$ consume $100 \mathrm{~g}$ food/d (Heinz et al.1989). Therefore, it was assumed that a $1.25 \mathrm{~kg}$ black duck would consume $125 \mathrm{~g}$ food $/ \mathrm{d}$.

Exposure Duration: $10 \mathrm{mo}$. ( $>10$ weeks and during a critical lifestage $=$ chronic). Endpoint: Exposure Route: Dosage: reproduction oral in diet two dose levels: 10 and $50 \mathrm{ppm} \mathrm{Cr}^{+3}$ in diet; NOAEL $=10 \mathrm{ppm}$

Calculations:

$$
\left[\frac{10 \mathrm{mg} \mathrm{Cr} \mathrm{r}^{3}}{\mathrm{~kg} \mathrm{food}} \times \frac{125 \mathrm{~g} \mathrm{food}}{\text { day }} \times \frac{1 \mathrm{~kg}}{1000 \mathrm{~g}}\right] / 1.25 \mathrm{~kg} \mathrm{BW}=1 \mathrm{mg} / \mathrm{kg} / \mathrm{d}
$$

Comments: Because no significant differences were observed at the $10 \mathrm{ppm} \mathrm{Cr}^{+3}$ dose level and the study considered exposure throughout a critical lifestage (reproduction), this dose was considered to be a chronic NOAEL.

Final NOAEL: $1 \mathrm{mg} / \mathrm{kg} / \mathrm{d}$

$\begin{array}{cc}\text { Compound: } & \text { Copper } \\ \text { Form: } & \text { Copper Sulfate } \\ \text { Reference: } & \text { Aulerich et al. } 1982 \\ \text { Test Species: } & \text { Mink }\end{array}$

Body weight: $1.0 \mathrm{~kg}$ (EPA 1993e)

food consumption: $0.137 \mathrm{~kg} / \mathrm{d}$ (Bleavins and Aulerich 1981)

Exposure Duration: $357 \mathrm{~d}$ (during a critical lifestage = chronic).

Endpoint:

Exposure Route:

reproduction

Dosage:

oral in diet

four dose levels:

$25,50,100$, and $200 \mathrm{ppm} \mathrm{Cu}$ supplemental $+60.5 \mathrm{ppm} \mathrm{Cu}$

in base feed; NOAEL $=85.5 \mathrm{ppm} \mathrm{Cu}$ (supplement + base)

Calculations:

$$
\left(\frac{85.5 \mathrm{mg} \mathrm{Cu}}{\mathrm{kg} \text { food }} \times \frac{137 \mathrm{~g} \mathrm{food}}{\text { day }} \times \frac{1 \mathrm{~kg}}{1000 \mathrm{~g}}\right] / 1 \mathrm{~kg} \mathrm{BW}=11.71 \mathrm{mg} / \mathrm{kg} / \mathrm{d}
$$

Comments: Consumption of 50,100, and $200 \mathrm{ppm}$ supplemental $\mathrm{Cu}$ increased the percentage mortality of mink kits. Kit survivorship among the $25 \mathrm{ppm}$ supplemental $\mathrm{Cu}$ group was actual greater than the controls. Because this study was approximately one year in duration and considered exposure during reproduction, the $25 \mathrm{ppm}$ supplemental $\mathrm{Cu}(85.5$ $\mathrm{ppm}$ total $\mathrm{Cu}$ ) dose was considered to be a chronic NOAEL.

Final NOAEL: $11.71 \mathrm{mg} / \mathrm{kg} / \mathrm{d}$ 


\section{Compound: Copper \\ Form: $\quad$ Copper Oxide \\ Reference: Mehring et al. 1960 \\ Test Species: 1 day old chicks}

Body weight: $0.534 \mathrm{~kg}$ (mean $_{\delta+q}$ at 5 weeks; EPA 1988a)

food consumption: $0.044 \mathrm{~kg} / \mathrm{d}$ (calculated using allometric equation from EPA 1988a)

Exposure Duration: 10 weeks $(10$ weeks $=$ chronic $)$.

Endpoint: growth

Exposure Route: oral in diet

Dosage: eleven dose levels: $36.8,52.0,73.5,104.0,147.1,208.0,294.1,403,570,749$, and $1180 \mathrm{ppm}$ total $\mathrm{Cu}$; NOAEL $=403 \mathrm{ppm}$ total $\mathrm{Cu}$

\section{Calculations:}

$$
\left(\frac{403 \mathrm{mg} \mathrm{Cu}}{\mathrm{kg} \text { food }} \times \frac{44 \mathrm{~g} \text { food }}{\text { day }} \times \frac{1 \mathrm{~kg}}{1000 \mathrm{~g}}\right) / 0.534 \mathrm{~kg} \mathrm{BW}=33.21 \mathrm{mg} / \mathrm{kg} / \mathrm{d}
$$

Comments: Consumption of $\mathrm{Cu}$ up to $403 \mathrm{ppm}$ had no effect of growth of chicks. Because this study was 10 weeks in duration, the $403 \mathrm{ppm} \mathrm{Cu}$ dose was considered to be a chronic NOAEL. To estimate daily $\mathrm{Cu}$ intake throughout the 10 week study period, food consumption of 5-week-old chicks was calculated. While this value will over- and underestimate food consumption by younger and older chicks, it was assumed to approximate food consumption throughout the entire 10 week study.

Final NOAEL: $33.21 \mathrm{mg} / \mathrm{kg} / \mathrm{d}$

\section{Compound: $\quad o$-Cresol}

Form: not applicable

Reference: Hornshaw et al. 1986

Test Species: Mink

Body weight: $1.0 \mathrm{~kg}$ (EPA 1993e)

food consumption: $0.137 \mathrm{~kg} / \mathrm{d}$ (Bleavins and Aulerich 1981)

Exposure Duration: 6 months (during a critical lifestage $=$ chronic).

Endpoint: reproduction

Exposure Route: oral in diet

Dosage: three dose levels:

Calculations:

$$
100,400 \text {, and } 1600 \mathrm{ppm} ; \mathrm{NOAEL}=1600 \mathrm{ppm}
$$

$$
\left[\frac{1600 \mathrm{mg} \mathrm{o} \text {-Cresol }}{\mathrm{kg} \text { food }} \times \frac{137 \mathrm{~g} \text { food }}{\text { day }} \times \frac{1 \mathrm{~kg}}{1000 \mathrm{~g}}\right] / 1 \mathrm{~kg} \mathrm{BW}=216.2 \mathrm{mg} / \mathrm{kg} / \mathrm{d}
$$


Comments: No adverse effects were observed at any dose level. Because this study considered exposure during reproduction, the maximum dose was considered to be a chronic NOAEL.

Final NOAEL: $216.2 \mathrm{mg} / \mathrm{kg} / \mathrm{d}$

\author{
Compound: $\quad$ Cyanide \\ Form: Potassium Cyanide \\ Reference: $\quad$ Tewe and Maner 1981 \\ Test Species: Rat
}

Body weight: $0.273 \mathrm{~kg}$ (from study)

Food Consumption: $0.0375 \mathrm{~kg} / \mathrm{d}$ (from study)

Exposure Duration: gestation and lactation (during a critical lifestage $=$ chronic).

Endpoint: reproduction

Exposure Route: oral in diet

Dosage:

one dose level:

$500 \mathrm{ppm} \mathrm{CN}=$ LOAEL

No effects observed at either dose level.

Calculations:

$$
\left(\frac{500 \mathrm{mg} \mathrm{CN}}{\mathrm{kg} \mathrm{food}} \times \frac{37.5 \mathrm{~g} \mathrm{food}}{\text { day }} \times \frac{1 \mathrm{~kg}}{1000 \mathrm{~g}}\right) / 0.273 \mathrm{~kg} \mathrm{BW}=68.7 \mathrm{mg} / \mathrm{kg} / \mathrm{d}
$$

Comments: Because consumption of $500 \mathrm{ppm} \mathrm{CN}$ reduced offspring growth and the study considered exposure throughout a critical lifestage (reproduction), this dose was considered to be a chronic LOAEL. To estimate the chronic NOAEL, the chronic NOAEL was multiplied by a LOAEL-NOAEL uncertainty factor of 0.1 .

Final NOAEL: $6.87 \mathrm{mg} / \mathrm{kg} / \mathrm{d}$

\title{
Compound: DDT \\ Form: not applicable \\ Reference: $\quad$ Fitzhugh 1948 \\ Test Species: Rat
}

Body weight: $0.35 \mathrm{~kg}$ (EPA 1988a)

Food Consumption: $0.028 \mathrm{~kg} / \mathrm{d}$ (calculated using allometric equation from EPA 1988a)

Exposure Duration: 2 yr $(>1 \mathrm{yr}$ and during a critical lifestage $=$ chronic)

Endpoint:

Exposure Route:

Dosage: reproduction,

oral in diet

four dose levels:

$10,50,100$, and $600 \mathrm{ppm} ;$ NOAEL $=10 \mathrm{ppm}$ 
Calculations:

$$
\left(\frac{10 \mathrm{mg} \mathrm{DDT}}{\mathrm{kg} \text { food }} \times \frac{28 \mathrm{~g} \text { food }}{\text { day }} \times \frac{1 \mathrm{~kg}}{1000 \mathrm{~g}}\right) / 0.35 \mathrm{~kg} \mathrm{BW}=0.8 \mathrm{mg} / \mathrm{kg} / \mathrm{d}
$$

Comments: While consumption of $50 \mathrm{ppm}$ or more DDT in the diet reduced the number of young produced, no adverse effects were observed at the $10 \mathrm{ppm}$ DDT dose level. Because the study considered exposure throughout 2 years and reproduction, the $10 \mathrm{ppm}$ DDT dose was considered to be a chronic NOAEL.

Final NOAEL: $0.8 \mathrm{mg} / \mathrm{kg} / \mathrm{d}$

\section{Compound: $\quad$ DDT \\ Form: not applicable \\ Reference: Anderson et al. 1975 \\ Test Species: $\quad$ Brown Pelican}

Body weight: $3.5 \mathrm{~kg}$ (Dunning 1984)

Food Consumption: $0.66 \mathrm{~kg} / \mathrm{d}$ (EPA 1993e)

Exposure Duration: 5 yr $(>1$ yr and during a critical lifestage $=$ chronic $)$

Endpoint:

Exposure Route:

Dosage: reproduction,

oral in diet

one dose level:

$0.15 \mathrm{ppm}$ DDT; LOAEL $=0.15 \mathrm{ppm}$

Calculations:

$$
\left(\frac{0.15 \mathrm{mg} \mathrm{DDT}}{\mathrm{kg} \text { food }} \times \frac{660 \mathrm{~g} \text { food }}{d a y} \times \frac{1 \mathrm{~kg}}{1000 \mathrm{~g}}\right) / 3.5 \mathrm{~kg} \mathrm{BW}=0.0028 \mathrm{mg} / \mathrm{kg} / \mathrm{d}
$$

Comments: Anderson et al. (1975) studied the reproductive success of pelicans from 1969 through 1974. During this time, DDT residues in anchovies, their primary food, declined from $4.27 \mathrm{ppm}$ (wet weight) to $0.15 \mathrm{ppm}$ (wet weight). While reproductive success improved from 1969 to 1974 , in 1974 the fledgling rate was still $30 \%$ below that needed to maintain a stable population. Because this study was long-term and considered reproductive effects in a wildlife species, EPA (1993) judged this study to be the most appropriate to evaluate DDT effects to avian wildlife. Therefore the $0.15 \mathrm{ppm}$ DDT value was considered to be a chronic LOAEL. To estimate the chronic NOAEL, the chronic NOAEL was multiplied by a LOAEL-NOAEL uncertainty factor of 0.1 .

Final NOAEL: $0.00028 \mathrm{mg} / \mathrm{kg} / \mathrm{d}$

$\begin{array}{cl}\text { Compound: } & \text { 1,2,-Dichloroethane } \\ \text { Form: } & \text { not applicable } \\ \text { Reference: } & \text { Lane et al. } 1982\end{array}$


Test Species: $\quad$ Mouse

Body weight: $0.035 \mathrm{~kg}$ (from study)

Water Consumption: $6 \mathrm{~mL} / \mathrm{d}$ (from study)

Exposure Duration: 2 generations $(>1 \mathrm{yr}$ and during a critical lifestage $=$ chronic).

Endpoint: reproduction

Exposure Route: oral in water

Dosage: three dose levels:

5,15 , and $50 \mathrm{mg} / \mathrm{kg} / \mathrm{d}$

No effects observed at any dose level.

Calculations: not applicable

Comments: Because no significant differences were observed at any dose level and the study considered exposure throughout 2 generations including critical lifestages (reproduction), the maximum dose was considered to be a chronic NOAEL.

Final NOAEL: $\quad 50 \mathrm{mg} / \mathrm{kg} / \mathrm{d}$.

Compound: $\quad 1,2$,-Dichloroethane

Form: not applicable

Reference: Alumot at al. 1976b

Test Species: Chicken

Body weight: $1.6 \mathrm{~kg}$ (mean ( $_{\delta+8}$ from study)

Food Consumption: $0.11 \mathrm{~kg} / \mathrm{d}$ (calculated using allometric equation from EPA 1988a)

Exposure Duration: 2 yr $(>10 \mathrm{wk}$ and during a critical lifestage $=$ chronic $)$.

Endpoint: reproduction

Exposure Route: oral in diet

Dosage: two dose levels:

250 and $500 \mathrm{ppm} ; \mathrm{NOAEL}=250 \mathrm{ppm}$

\section{Calculations:}

$$
\left(\frac{250 \mathrm{mg} 1,2 \text { Dichloroethane }}{\mathrm{kg} \text { food }} \times \frac{0.11 \mathrm{~kg} \text { food }}{\text { day }}\right) / 1.6 \mathrm{~kg} \mathrm{BW}=17.2 \mathrm{mg} / \mathrm{kg} / \mathrm{d}
$$

Comments: Because no significant differences were observed at the $250 \mathrm{ppm}$ dose level and the study considered exposure throughout 2 years including critical lifestages (reproduction), this dose was considered to be a chronic NOAEL.

Final NOAEL: $17.2 \mathrm{mg} / \mathrm{kg} / \mathrm{d}$

Compound: 1,1-Dichloroethylene

Form: not applicable

Reference: Quast et al. 1983

Test Species: Rat

Body weight: $0.35 \mathrm{~kg}$ (EPA 1988a) 
Exposure Duration: 2 years $(>1 \mathrm{yr}=$ chronic).

Endpoint: mortality, body weight, blood chemistry, liver histology

Exposure Route: oral in water

Dosage: three dose levels:

7,10 , and $20 \mathrm{mg} / \mathrm{kg} / \mathrm{d}$ (males) and

Calculations: 9,14 , and $30 \mathrm{mg} / \mathrm{kg} / \mathrm{d}$ (females); NOAEL $=30 \mathrm{mg} / \mathrm{kg} / \mathrm{d}$ not applicable

Comments: The only treatment-related effect observed were microscopic hepatic lesions. These were evident among females at all dose levels and among males only at the highest dose level. No other treatment effects were observed. Because the relationship of hepatic lesions to potential population effects is unknown and no other effects were observed, the maximum dose, $30 \mathrm{mg} / \mathrm{kg} / \mathrm{d}$ was considered a chronic NOAEL.

Final NOAEL: $30 \mathrm{mg} / \mathrm{kg} / \mathrm{d}$

Compound: 1,1-Dichloroethylene

Form: not applicable

Reference: Quast et al. 1983

Test Species: $\quad \operatorname{dog}$ (beagle)

Body weight: $10 \mathrm{~kg}$ (EPA 1988a)

Exposure Duration: $97 \mathrm{~d}(<1 \mathrm{yr}$ and not during a critical lifestage $=$ subchronic).

Endpoint:

Exposure Route: daily oral capsules

Dosage:

Calculations: three dose levels:

$6.25,12.5$, and $25 \mathrm{mg} / \mathrm{kg} / \mathrm{d} ; \mathrm{NOAEL}=25 \mathrm{mg} / \mathrm{kg} / \mathrm{d}$ not applicable

Comments: No adverse effects were observed among any of the treatments, therefore the maximum dose, $25 \mathrm{mg} / \mathrm{kg} / \mathrm{d}$ was considered a subchronic NOAEL. A chronic NOAEL was estimated by multiplying the subchronic NOAEL by a subchronic-chronic uncertainty factor of 0.1 .

Final NOAEL: $2.5 \mathrm{mg} / \mathrm{kg} / \mathrm{d}$

Compound: $\quad$ 1,2-Dichloroethylene

Form: not applicable

Reference: $\quad$ Palmer et al. 1979

Test Species: Mouse

Body weight: $0.03 \mathrm{~kg}$ (EPA 1988a)

Exposure Duration: $90 \mathrm{~d}(<1 \mathrm{yr}$ and not during a critical lifestage $=$ subchronic).

Endpoint:

Exposure Route: oral in water 
Dosage: $\quad$ three dose levels:

$16.8,175$, and $387 \mathrm{mg} / \mathrm{kg} / \mathrm{d}$ (Males)

$22.6,224$, and $452 \mathrm{mg} / \mathrm{kg} / \mathrm{d}$ (Females)

NOAEL $=452 \mathrm{mg} / \mathrm{kg} / \mathrm{d}$

Calculations: not applicable

Comments: Exposure to $387 \mathrm{mg} / \mathrm{kg} / \mathrm{d}$ 1,2-Dichloroethylene reduced glutathione levels in males and all dose levels reduced aniline hydroxylase activity in females. No other treatment effects were observed. Because the relationship of enzyme levels to potential population effects is unknown and no other effects were observed, the maximum dose, 452 $\mathrm{mg} / \mathrm{kg} / \mathrm{d}$ was considered a subchronic NOAEL. To estimate the chronic NOAEL, the subchronic NOAEL was multiplied by a subchronic-chronic uncertainty factor of 0.1 .

Final NOAEL: $45.2 \mathrm{mg} / \mathrm{kg} / \mathrm{d}$

\section{Compound: Dieldrin}

Form: not applicable

Reference: $\quad$ Treon and Cleveland 1955

Test Species: Rat

Body weight: $0.35 \mathrm{~kg}$ (EPA 1988a)

Food Consumption: $0.028 \mathrm{~kg} / \mathrm{d}$ (calculated using allometric equation from EPA 1988a)

Exposure Duration: 3 generations $(>1 \mathrm{yr}$ and during a critical lifestage $=$ chronic).

Endpoint:

Exposure Route:

Dosage:

Calculations:

$$
\left(\frac{2.5 \mathrm{mg} \text { Dieldrin }}{\mathrm{kg} \text { food }} \times \frac{28 \mathrm{~g} \text { food }}{\text { day }} \times \frac{1 \mathrm{~kg}}{1000 \mathrm{~g}}\right) / 0.35 \mathrm{~kg} \mathrm{BW}=0.2 \mathrm{mg} / \mathrm{kg} / \mathrm{d}
$$

Comments: Because Dieldrin at $2.5 \mathrm{ppm}$ in the diet reduced the number of pregnancies in rats and the study considered exposure throughout 3 generations including critical lifestages (reproduction), this dose was considered to be a chronic LOAEL. A chronic NOAEL was estimated by multiplying the chronic LOAEL by a LOAEL-NOAEL uncertainty factor of 0.1 .

Final NOAEL: $0.02 \mathrm{mg} / \mathrm{kg} / \mathrm{d}$

Compound: Dieldrin

Form: not applicable

Reference: Mendenhall et al. 1983 
Test Species: $\quad$ Barn Owl

Body weight (BW): $0.466 \mathrm{~kg}$ (mean $_{\delta+q} ;$ Johnsgard 1988)

Food Consumption: wild birds 100-150 g/d ; 50-75 g/d captive (Johnsgard 1988). Used median captive food consumption value: $62.5 \mathrm{~g} / \mathrm{d}$

Exposure Duration: 2 yrs $(>10$ weeks and during a critical lifestage $=$ chronic).

Endpoint:

Exposure Route: oral in diet

Dosage: reproduction

Calculations:

Only 1 dose level applied: 0.58 ppm NOAEL

$\left(\frac{0.58 \mathrm{mg} \text { Dieldrin }}{\mathrm{kg} \text { food }} \times \frac{62.5 \mathrm{~g} \text { food }}{\text { day }} \times \frac{1 \mathrm{~kg}}{1000 \mathrm{~g}}\right) / 0.466 \mathrm{~kg} \mathrm{BW}=0.077 \mathrm{mg} / \mathrm{kg} / \mathrm{d}$

Comments: While $0.58 \mathrm{ppm}$ Dieldrin in the diet produced a slight but significant reduction in eggshell thickness, no significant effect on no. eggs laid/pair, no. eggs hatched/pair, \% eggs broken, embryo or nestling mortality was observed. Therefore this dose was considered to be a chronic NOAEL.

Final NOAEL: $0.077 \mathrm{mg} / \mathrm{kg} / \mathrm{d}$

Compound: $\quad$ Diethylphthalate (DEP)

Form: not applicable

Reference: Lamb et al. 1987

Test Species: Mouse

Body weight: $0.03 \mathrm{~kg}$ (EPA 1988a)

Food Consumption: $0.0055 \mathrm{~kg} / \mathrm{d}$

(calculated using allometric equation from EPA 1988a)

Exposure Duration: $105 \mathrm{~d}$ (during a critical lifestage $=$ chronic).

Endpoint: reproduction

Exposure Route: oral in diet

Dosage: three dose levels:

$0.25 \%, 1.25 \%$ and $2.5 \%$ of diet;

NOAEL $=2.5 \%=25000 \mathrm{mg} / \mathrm{kg}$

Calculations:

$$
\left(\frac{25000 \mathrm{mg} \mathrm{DEP}}{\mathrm{kg} \text { food }} \times \frac{5.5 \mathrm{~g} \text { food }}{\text { day }} \times \frac{1 \mathrm{~kg}}{1000 \mathrm{~g}}\right) / 0.03 \mathrm{~kg} \mathrm{BW}=4583 \mathrm{mg} / \mathrm{kg} / \mathrm{d}
$$

Comments: No significant reproductive effects were observed among mice in any of the treatment groups. Because the study considered exposure during a critical lifestage, the maximum dose was considered to be a chronic NOAEL.

Final NOAEL: $4583 \mathrm{mg} / \mathrm{kg} / \mathrm{d}$ 
Compound: Di-n-butyl phthalate (DBP)

Form: not applicable

Reference: Lamb et al. 1987

Test Species: Mouse

Body weight: $0.03 \mathrm{~kg}$ (EPA 1988a)

Food Consumption: $0.0055 \mathrm{~kg} / \mathrm{d}$

(calculated using allometric equation from EPA 1988a)

Exposure Duration: $105 \mathrm{~d}$ (during a critical lifestage = chronic).

Endpoint: reproduction

Exposure Route: oral in diet

Dosage: three dose levels: $0.03 \%, 0.3 \%$ and $1 \%$ of diet; NOAEL $=0.3 \%=3000 \mathrm{mg} / \mathrm{kg}$

Calculations:

$$
\left(\frac{3000 \mathrm{mg} \mathrm{DBP}}{\mathrm{kg} \mathrm{food}} \times \frac{5.5 \mathrm{~g} \text { food }}{\text { day }} \times \frac{1 \mathrm{~kg}}{1000 \mathrm{~g}}\right] / 0.03 \mathrm{~kg} \mathrm{BW}=550 \mathrm{mg} / \mathrm{kg} / \mathrm{d}
$$

Comments: While significant reproductive effects were observed among mice on diet containing $1 \%$ DBP, no adverse effects were observed among either the $0.03 \%$ or $0.3 \%$ dose groups. Because the study considered exposure during a critical lifestage, the $0.3 \%$ dose was considered to be a chronic NOAEL.

Final NOAEL: $550 \mathrm{mg} / \mathrm{kg} / \mathrm{d}$

Compound: $\quad$ Di-n-butyl phthalate (DBP)

Form: not applicable

Reference: $\quad$ Peakall 1974

Test Species: $\quad$ Ringed Dove

Body weight: $0.155 \mathrm{~kg}$ (Terres 1980)

Food Consumption: $0.01727 \mathrm{~kg} / \mathrm{d}$ (calculated using allometric equation from

Nagy 1987)

Exposure Duration: 4 weeks (during a critical lifestage $=$ chronic).

Endpoint: reproduction

Exposure Route: oral in diet

Dosage:

one dose level:

$10 \mathrm{ppm}=\mathrm{LOAEL}$

Calculations:

$$
\left[\frac{10 \mathrm{mg} \mathrm{DBP}}{\mathrm{kg} \mathrm{food}} \times \frac{17.27 \mathrm{~g} \text { food }}{\text { day }} \times \frac{1 \mathrm{~kg}}{1000 \mathrm{~g}}\right] / 0.155 \mathrm{~kg} \mathrm{BW}=1.11 \mathrm{mg} / \mathrm{kg} / \mathrm{d}
$$


Comments: Eggshell thickness and water permeability of the shell was reduced among doves on diets containing $10 \mathrm{ppm}$ DBP. Because the study considered exposure during a critical lifestage the $10 \mathrm{ppm}$ dose was considered to be a chronic LOAEL. A chronic NOAEL was estimated by multiplying the chronic LOAEL by a LOAEL-NOAEL uncertainty factor of 0.1 .

Final NOAEL: $0.111 \mathrm{mg} / \mathrm{kg} / \mathrm{d}$
Compound: Di-n-hexylphthalate (DHP)
Form: not applicable
Reference: Lamb et al. 1987
Test Species: Mouse

Body weight: $0.03 \mathrm{~kg}$ (EPA 1988a)

Food Consumption: $0.0055 \mathrm{~kg} / \mathrm{d}$

(calculated using allometric equation from EPA 1988a)

Exposure Duration: $105 \mathrm{~d}$ (during a critical lifestage = chronic)..

Endpoint: reproduction

Exposure Route: oral in diet

Dosage:

three dose levels:

$0.3 \%, 0.6 \%$ and $1.2 \%$ of diet;

LOAEL $=0.3 \%=3000 \mathrm{mg} / \mathrm{kg}$

Calculations:

$$
\left(\frac{3000 \mathrm{mg} \mathrm{DHP}}{\mathrm{kg} \text { food }} \times \frac{5.5 \mathrm{~g} \text { food }}{\text { day }} \times \frac{1 \mathrm{~kg}}{1000 \mathrm{~g}}\right) / 0.03 \mathrm{~kg} \mathrm{BW}=550 \mathrm{mg} / \mathrm{kg} / \mathrm{d}
$$

Comments: Significant reproductive effects were observed among mice on all diets. Because the study considered exposure during a critical lifestage, the $0.3 \%$ dose was considered to be a chronic LOAEL. A chronic NOAEL was estimated by multiplying the chronic LOAEL by a LOAEL-NOAEL uncertainty factor of 0.1 .

Final NOAEL: $55 \mathrm{mg} / \mathrm{kg} / \mathrm{d}$

\author{
Compound: $\quad$ 1,4-Dioxane \\ Form: not applicable \\ Reference: Giavini et al. 1985 \\ Test Species: rat \\ Body weight: $0.35 \mathrm{~kg}$ (EPA 1988a) \\ Exposure Duration: days 6-15 of gestation (during a critical lifestage $=$ chronic). \\ Endpoint: reproduction \\ Exposure Route: oral intubation \\ Dosage: \\ three dose levels: \\ $0.25,0.5$, and $1.0 \mathrm{mg} / \mathrm{kg} / \mathrm{d} ;$ NOAEL $=0.5 \mathrm{mg} / \mathrm{kg} / \mathrm{d}$ \\ Calculations: not applicable
}


Comments: Maternal toxicity and reduced fetal weights were observed among rats receiving the $1.0 \mathrm{mg} / \mathrm{kg} / \mathrm{d}$ dose. No adverse effects were observed among the other treatments. Because the study considered exposure during a critical lifestage, the $0.5 \mathrm{mg} / \mathrm{kg} / \mathrm{d}$ was considered to be a chronic NOAEL.

Final NOAEL: $0.5 \mathrm{mg} / \mathrm{kg} / \mathrm{d}$

$\begin{array}{ll}\text { Compound: } & \text { Endosulfan } \\ \text { Form: } & \text { not applicable } \\ \text { Reference: } & \text { Dikshith et al. } 1984 \\ \text { Test Species: } & \text { Rat } \\ & \text { Body weight: } 0.35 \mathrm{~kg} \text { (EPA }\end{array}$

Food Consumption: $0.028 \mathrm{~kg} / \mathrm{d}$ (calculated using allometric equation from EPA 1988a)

Exposure Duration: 30 days

( $<1 \mathrm{yr}$ and not during a critical lifestage $=$ subchronic $)$.

Endpoint: reproduction, blood chemistry

Exposure Route: oral intubation

Dosage: three dose levels per sex: male: $0.75,2.5$, and $5.0 \mathrm{mg} / \mathrm{kg} / \mathrm{d}$ female $0.25,0.75$, and $1.5 \mathrm{mg} / \mathrm{kg} / \mathrm{d}$

Calculations: not applicable

Comments: Male and female rats were dosed for 30 days at the three respective dose levels, then one male and two females from the following groups were paired and allowed to

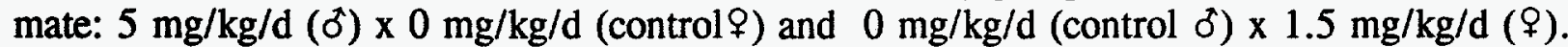
No adverse effects were observed for any dose level. Because it was assumed that adverse reproductive effects were more likely to be observed in exposed females than males, and because the study was $<1 \mathrm{yr}$ in duration and did not include a critical lifestage (exposure was discontinued prior to gestation), the $1.5 \mathrm{mg} / \mathrm{kg} / \mathrm{d}$ dose was considered a subchronic NOAEL. A chronic NOAEL was estimated by multiplying the subchronic NOAEL by a subchronic-chronic uncertainty factor of 0.1 .

Final NOAEL: $0.15 \mathrm{mg} / \mathrm{kg} / \mathrm{d}$

Compound: Endosulfan

Form: not applicable

Reference: $\quad$ Abiola 1992

Test Species: Gray Partridge

Body weight: $0.400 \mathrm{~kg}$ (from study)

Food Consumption: $0.032 \mathrm{~kg} / \mathrm{d}$ (calculated using allometric equation from Nagy 1987)

Exposure Duration: 4 weeks (during a critical lifestage $=$ chronic).

Endpoint: reproduction

Exposure Route: oral in diet 
Dosage: three dose levels:

Calculations:

$$
5,25,125 \mathrm{ppm} ; \text { NOAEL }=125 \mathrm{ppm}
$$

$$
\left(\frac{125 \mathrm{mg} \text { Endosulfan }}{\mathrm{kg} \text { food }} \times \frac{32 \mathrm{~g} \text { food }}{\text { day }} \times \frac{1 \mathrm{~kg}}{1000 \mathrm{~g}}\right] / 0.400 \mathrm{~kg} \mathrm{BW}=10 \mathrm{mg} / \mathrm{kg} / \mathrm{d}
$$

Comments: No adverse effects were observed at any dose level. Because exposure occurred during reproduction, the maximum dose was considered a chronic NOAEL.

Final NOAEL: $10 \mathrm{mg} / \mathrm{kg} / \mathrm{d}$

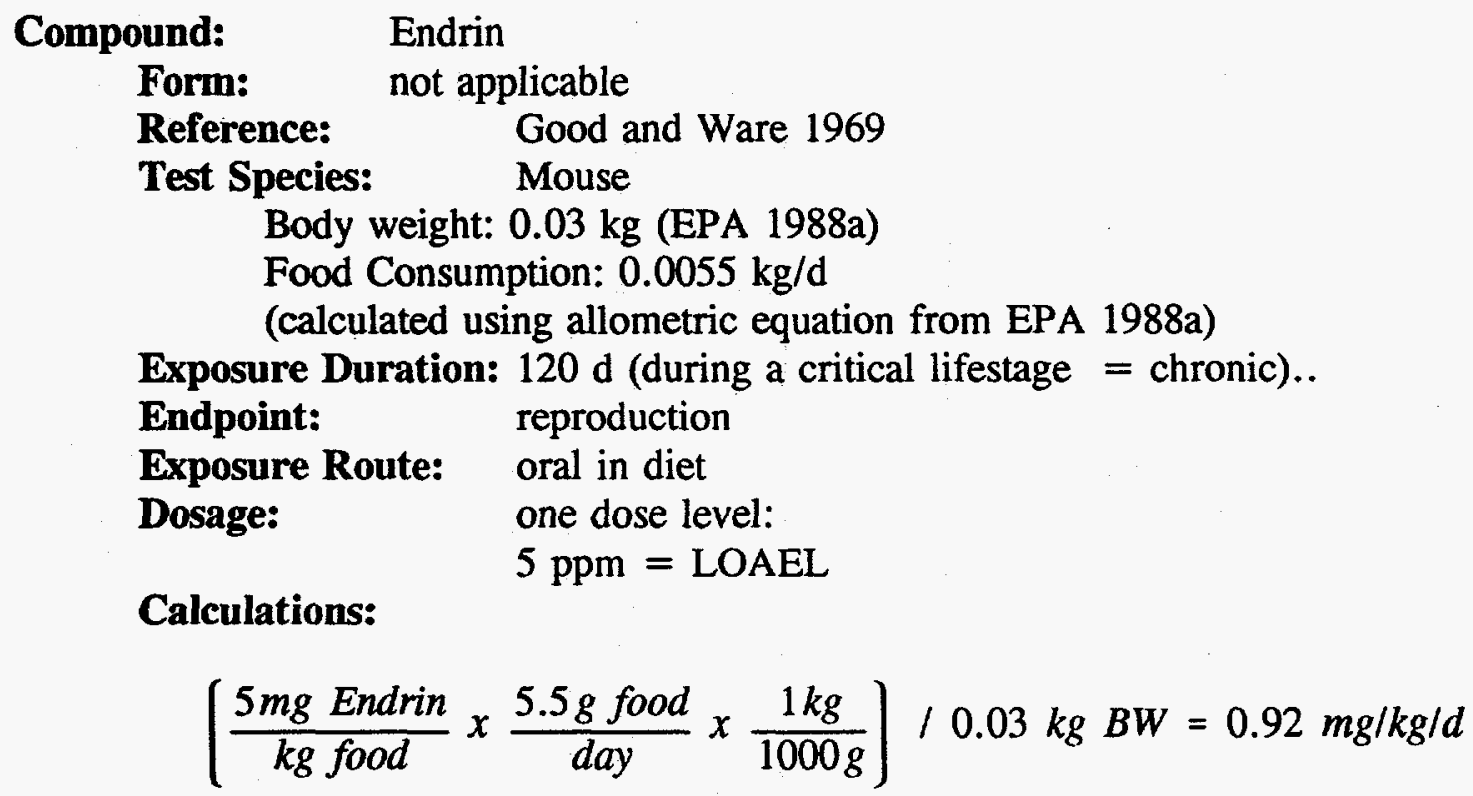

Comments: Significant reproductive effects (reduced parental survival, litter size, and number of young/d) were observed among mice fed diets containing $5 \mathrm{ppm}$ Endrin. Because the study considered exposure during a critical lifestage, this dose was considered to be a chronic LOAEL. A chronic NOAEL was estimated by multiplying the chronic LOAEL by a LOAEL-NOAEL uncertainty factor of 0.1 .

Final NOAEL: $0.092 \mathrm{mg} / \mathrm{kg} / \mathrm{d}$

\section{Compound: $\quad$ Endrin \\ Form: not applicable \\ Reference: $\quad$ Spann et al. 1986 \\ Test Species: Mallard duck}

Body weight: $1.15 \mathrm{~kg}$ (from study)

Food Consumption: Mallard ducks, weighing $1 \mathrm{~kg}$ consume $100 \mathrm{~g}$ food/d (Heinz et al.1989). Therefore, it was assumed that a $1.15 \mathrm{~kg}$ Mallard duck would consume $115 \mathrm{~g}$ food $/ \mathrm{d}$. 
Exposure Duration: $>200 \mathrm{~d}$. ( $>10$ weeks and during a critical lifestage $=$ chronic). Endpoint: reproduction

Exposure Route: oral in diet

Dosage:

two dose levels:

1 and $3 \mathrm{ppm}$ Endrin in diet; NOAEL $=3 \mathrm{ppm}$

Calculations:

$$
\left[\frac{3 \mathrm{mg} \text { Endrin }}{\mathrm{kg} \text { food }} \times \frac{115 \mathrm{~g} \text { food }}{\mathrm{day}} \times \frac{1 \mathrm{~kg}}{1000 \mathrm{~g}}\right] / 1.15 \mathrm{~kg} \mathrm{BW}=0.3 \mathrm{mg} / \mathrm{kg} / \mathrm{d}
$$

Comments: While the authors state that birds receiving the $3 \mathrm{ppm}$ dose appeared to reproduce more poorly than controls, this difference was not significant. Because no significant differences were observed at the $3 \mathrm{ppm}$ dose level and the study considered exposure throughout a critical lifestage (reproduction), this dose was considered to be a chronic NOAEL.

Final NOAEL: $0.3 \mathrm{mg} / \mathrm{kg} / \mathrm{d}$

\title{
Compound: Ethanol
}

Form: not applicable

Reference: $\quad$ Mankes et al. 1982

Test Species: $\quad$ Rat

$$
\text { Body weight: } 0.35 \mathrm{~kg} \text { (EPA 1988a) }
$$

Exposure Duration: through gestation (during a critical lifestage $=$ chronic).

Endpoint:

reproduction

Exposure Route: oral intubation

Dosage:

Calculations: two dose levels: 0.4 and $4.0 \mathrm{ml} / \mathrm{kg} / \mathrm{d} ; \mathrm{LOAEL}=0.4 \mathrm{ml} / \mathrm{kg} / \mathrm{d}$ density of ethanol $=0.798 \mathrm{~g} / \mathrm{mL}$ (Merck 1976)

$$
\left(\frac{0.4 \mathrm{~mL} \text { Ethanol }}{\mathrm{kgBW}} \times \frac{0.798 \mathrm{~g} \text { Ethanol }}{\mathrm{mL} \text { Ethanol }} \times \frac{1000 \mathrm{mg}}{1 \mathrm{~g}}\right)=319 \mathrm{mg} / \mathrm{kg} / \mathrm{d}
$$

Comments: While $0.4 \mathrm{ml}$ Ethanol $/ \mathrm{kg} / \mathrm{d}$ had no effect on most reproductive parameters, the incidence of malformed fetuses was significantly increased at this dose level. Therefore this dose was considered to be a chronic LOAEL. To estimate the chronic NOAEL, the LOAEL was multiplied by a LOAEL-NOAEL uncertainty factor of 0.1 . Final NOAEL: $31.9 \mathrm{mg} / \mathrm{kg} / \mathrm{d}$

\author{
Compound: $\quad$ Ethyl Acetate \\ Form: not applicable \\ Reference: $\quad$ EPA 1986d \\ Test Species: Rat \\ Body weight: $0.35 \mathrm{~kg}$ (EPA 1988a)
}




\section{A-36}

Exposure Duration: 90 days ( $<1$ yr and not during a critical lifestage $=$ subchronic).

Endpoint: mortality and weight loss

Exposure Route: oral intubation

Dosage:

Calculations: three dose levels: 300,900 , and $3600 \mathrm{mg} / \mathrm{kg} / \mathrm{d} ;$ NOAEL $=900 \mathrm{mg} / \mathrm{kg} / \mathrm{d}$ not applicable

Comments: While Ethyl Acetate at $3600 \mathrm{mg} / \mathrm{kg} / \mathrm{d}$ reduced body and organ weights and food consumption by male rats, no effects were observed at the $900 \mathrm{mg} / \mathrm{kg} / \mathrm{d}$ dose level. Because the study was 90 days in duration and did not consider exposure during critical lifestages, the $900 \mathrm{mg} / \mathrm{kg} / \mathrm{d}$ dose was considered to be a chronic LOAEL. A chronic NOAEL was estimated by multiplying the chronic LOAEL by a LOAEL-NOAEL uncertainty factor of 0.1 .

Final NOAEL: $90 \mathrm{mg} / \mathrm{kg} / \mathrm{d}$

\begin{tabular}{lll} 
Compound: & \multicolumn{2}{l}{ Fluoride } \\
Form: & NaF & \\
Reference: & $\cdot \quad$ Aulerich et al. 1987 \\
Test Species: & $\quad$ Mink
\end{tabular}

Body weight: $1.0 \mathrm{~kg}$ (EPA 1993e)

food consumption: $0.137 \mathrm{~kg} / \mathrm{d}$ (Bleavins and Aulerich 1981)

Exposure Duration: $382 \mathrm{~d}$ (during a critical lifestage $=$ chronic).

Endpoint:

Exposure Route:

Dosage:

reproduction

oral in diet

five dose levels:

$33,60,108,194$, and $350 \mathrm{ppm}$ supplemental $\mathrm{F}+35 \mathrm{ppm} \mathrm{F}$ in base diet; NOAEL $=194 \mathrm{ppm}+35 \mathrm{ppm}=229 \mathrm{ppm} \mathrm{F}$

Calculations:

$$
\left(\frac{229 \mathrm{mg} \mathrm{F}}{\mathrm{kg} \text { food }} \times \frac{137 \mathrm{~g} \mathrm{food}}{\text { day }} \times \frac{1 \mathrm{~kg}}{1000 \mathrm{~g}}\right) / 1 \mathrm{~kg} \mathrm{BW}=31.37 \mathrm{mg} / \mathrm{kg} / \mathrm{d}
$$

Comments: Fluoride up to $229 \mathrm{ppm}$ in mink diets had no adverse effects on reproduction; Survivorship of kits in the $385 \mathrm{ppm}(350+35 \mathrm{ppm})$ group was significantly reduced. Because $229 \mathrm{ppm} \mathrm{F}$ in the diet had no adverse effect and the study considered exposure over 382 days including critical lifestages (reproduction), this dose was considered to be a chronic NOAEL.

Final NOAEL: $31.37 \mathrm{mg} / \mathrm{kg} / \mathrm{d}$

\begin{tabular}{|c|c|}
\hline $\begin{array}{l}\text { Compound: } \\
\text { Form: }\end{array}$ & $\begin{array}{l}\text { Fluoride } \\
\text { NaF }\end{array}$ \\
\hline
\end{tabular}


Test Species: $\quad$ Screech Owl

Body weight: $0.181 \mathrm{~kg}$ (Dunning 1984)

food consumption: 1300-1700 g/month/pair (from study)

Daily food consumption was estimated as follows:

median food consumption $/$ month/pair $=1500 \mathrm{~g}$;

1 month $=30 \mathrm{~d}$;

Males and females consume equal amounts of food $=750 \mathrm{~g} / \mathrm{month}$

$750 \mathrm{~g} /$ month $\div 30 \mathrm{~d}=25 \mathrm{~g} / \mathrm{d}$

Exposure Duration: 5-6 months (during a critical lifestage $=$ chronic).

Endpoint:

Exposure Route:

Dosage: reproduction

oral in diet

two dose levels:

56.5 and $232 \mathrm{ppm}$ F; NOAEL $=56.5 \mathrm{ppm} \mathrm{F}$

\title{
Calculations:
}

$$
\left(\frac{56.5 \mathrm{mg} \mathrm{F}}{\mathrm{kg} \text { food }} \times \frac{25 \mathrm{~g} \text { food }}{\text { day }} \times \frac{1 \mathrm{~kg}}{1000 \mathrm{~g}}\right) / 0.181 \mathrm{~kg} \mathrm{BW}=7.8 \mathrm{mg} / \mathrm{kg} / \mathrm{d}
$$

Comments: Fertility and hatching success was significantly reduced by $232 \mathrm{ppm} F$ in the diet. Because $56.5 \mathrm{ppm} F$ in the diet had no adverse effect and the study considered exposure during reproduction, this dose was considered to be a chronic NOAEL.

Final NOAEL: $7.8 \mathrm{mg} / \mathrm{kg} / \mathrm{d}$

\author{
Compound: $\quad$ Formaldehyde \\ Form: not applicable \\ Reference: Hurni and Ohder 1973 \\ Test Species: dog (beagle) \\ Body weight: $12 \mathrm{~kg}$ (from study) \\ Exposure Duration: through gestation and lactation \\ Endpoint: \\ (during a critical lifestage $=$ chronic). \\ reproduction \\ Exposure Route: oral in diet \\ Dosage: \\ two dose levels: \\ 3.1 and $9.4 \mathrm{mg} / \mathrm{kg} / \mathrm{d} ; \mathrm{NOAEL}=9.4 \mathrm{mg} / \mathrm{kg} / \mathrm{d}$ \\ Calculations: not applicable \\ Comments: Because significant effects were not observed at any dose level, the 9.4 \\ $\mathrm{mg} / \mathrm{kg} / \mathrm{d}$ was considered to be a chronic NOAEL. \\ Final NOAEL: $9.4 \mathrm{mg} / \mathrm{kg} / \mathrm{d}$
}

\section{Compound: Heptachlor \\ Form: not applicable \\ Reference: $\quad$ Eisler 1968}


Test Species: Rat

Body weight: $0.35 \mathrm{~kg}$ (EPA 1988a)

Food Consumption: $0.028 \mathrm{~kg} / \mathrm{d}$ (calculated using allometric equation from EPA 1988a)

Exposure Duration: 3 generations ( $>1 \mathrm{yr}$ and during a critical lifestage $=$ chronic).

Endpoint:

Exposure Route: reproduction

Dosage: oral in diet four dose levels: $0.3,3,6$, and $10 \mathrm{ppm} ;$ NOAEL $=10 \mathrm{ppm}$

Calculations:

$$
\left(\frac{10 \mathrm{mg} \text { Heptachlor }}{\mathrm{kg} \mathrm{food}} \times \frac{28 \mathrm{~g} \text { food }}{\text { day }} \times \frac{1 \mathrm{~kg}}{1000 \mathrm{~g}}\right) / 0.35 \mathrm{~kg} \mathrm{BW}=0.8 \mathrm{mg} / \mathrm{kg} / \mathrm{d}
$$

Comments: Because significant effects were not observed at any dose level, the 10 ppm was considered to be a chronic NOAEL.

Final NOAEL: $0.8 \mathrm{mg} / \mathrm{kg} / \mathrm{d}$

Compound: $\quad 1,2,3,6,7,8$ - Hexachloro Dibenzofuran (HxDBF)

Form: not applicable

Reference: $\quad$ Poiger et al. 1989

Test Species: Rat

Body weight: $0.35 \mathrm{~kg}$ (EPA 1988a)

Food Consumption: $0.028 \mathrm{~kg} / \mathrm{d}$ (calculated using allometric equation from EPA 1988a)

Exposure Duration: 13 weeks

$(<1$ yr and not during a critical lifestage = subchronic $)$.

Endpoint: $\quad$ Body weight, organ weight, blood chemistry

Exposure Route: oral in diet

Dosage: three dose levels:

\section{Calculations:}

2,20 , and $200 \mathrm{ppb} ;$ NOAEL $=20 \mathrm{ppb}$

$$
\left(\frac{0.02 \mathrm{mg} \mathrm{H} \mathrm{HDBF}}{\mathrm{kg} \mathrm{food}} \times \frac{28 \mathrm{~g} \text { food }}{\text { day }} \times \frac{1 \mathrm{~kg}}{1000 \mathrm{~g}}\right) / 0.35 \mathrm{~kg} \mathrm{BW}=0.0016 \mathrm{mg} / \mathrm{kg} / \mathrm{d}
$$

Comments: Because rats exposed to $200 \mathrm{ppb} \mathrm{HxDBF}$ in the diet displayed reduced body, thymus and liver weights, while those in the $20 \mathrm{ppb}$ group did not, the $20 \mathrm{ppb}$ dose was considered to be a subchronic NOAEL. A chronic NOAEL was estimated by multiplying the subchronic NOAEL by a subchronic-chronic uncertainty factor of 0.1 .

Final NOAEL: $0.00016 \mathrm{mg} / \mathrm{kg} / \mathrm{d}$ 

Compound: Lead
Form: Lead Acetate
Reference: $\quad$ Azar et al. 1973
Test Species: Rat
Body weight: $0.35 \mathrm{~kg}$ (EPA 1988a)
Food Consumption: $0.028 \mathrm{~kg} / \mathrm{d}$ (calculated using allometric equation from EPA 1988a)
Exposure Duration: 3 generations $(>1 \mathrm{yr}$ and during a critical lifestage $=$ chronic).
Endpoint: reproduction
Exposure Route: oral in diet
Dosage:
five dose levels:
$10,50,100,1000$, and $2000 \mathrm{ppm} \mathrm{Pb} ; \mathrm{NOAEL}=100 \mathrm{ppm} \mathrm{Pb}$

\section{Calculations:}

$$
\left[\frac{100 \mathrm{mg} \mathrm{Pb}}{\mathrm{kg} \text { food }} \times \frac{28 g \text { food }}{\text { day }} \times \frac{1 \mathrm{~kg}}{1000 \mathrm{~g}}\right) / 0.35 \mathrm{~kg} \mathrm{BW}=8 \mathrm{mg} / \mathrm{kg} / \mathrm{d}
$$

Comments: While none of the $\mathrm{Pb}$ exposure levels studied affected the number of pregnancies, the number of live births, or other reproductive indices, $\mathrm{Pb}$ exposure of 1000 and $2000 \mathrm{ppm}$ resulted in reduced offspring weights and produced kidney damage in the young. Therefore the $100 \mathrm{ppm} \mathrm{Pb}$ dose was considered to be a chronic NOAEL.

Final NOAEL: $8 \mathrm{mg} / \mathrm{kg} / \mathrm{d}$

\section{Compound: Lead \\ Form: Metallic \\ Reference: $\quad$ Pattee 1984 \\ Test Species: $\quad$ American Kestrels}

Body weight: $0.130 \mathrm{~kg}$ ( mean $_{\delta+q} ;$ from study)

Food Consumption: Kenaga (1973) states that the congeneric European kestrel consumes $7.7 \%$ of body weight/d. Therefore, food consumption was assumed to be $0.077 \times 0.130 \mathrm{~kg}$ or $0.01 \mathrm{~kg} / \mathrm{d}$.

Exposure Duration: 7 months ( $>10$ weeks and during a critical lifestage $=$ chronic).

Endpoint: reproduction

Exposure Route: oral in diet

Dosage: two dose levels:

10 and $50 \mathrm{ppm} \mathrm{Pb} ;$ NOAEL $=50 \mathrm{ppm} \mathrm{Pb}$

Calculations:

$$
\left(\frac{50 \mathrm{mg} \mathrm{Pb}}{\mathrm{kg} \text { food }} \times \frac{10 \mathrm{~g} \text { food }}{\text { day }} \times \frac{1 \mathrm{~kg}}{1000 \mathrm{~g}}\right) / 0.13 \mathrm{~kg} \mathrm{BW}=3.85 \mathrm{mg} / \mathrm{kg} / \mathrm{d}
$$

Comments: Because significant effects were not observed at either dose levels and the study considered exposure over 7 months and throughout a critical lifestage 
(reproduction), the maximum dose was considered to be a chronic NOAEL.

Final NOAEL: $3.85 \mathrm{mg} / \mathrm{kg} / \mathrm{d}$

Compound: $\quad$ Lindane $(\gamma$-BHC)

Form: not applicable

Reference: $\quad$ Palmer et al. 1978

Test Species: Rat

Body weight: $0.35 \mathrm{~kg}$ (EPA 1988a)

Food Consumption: $0.028 \mathrm{~kg} / \mathrm{d}$ (calculated using allometric equation from EPA 1988a)

Exposure Duration: 3 generations ( $>1 \mathrm{yr}$ and during a critical lifestage $=$ chronic).

Endpoint: reproduction

Exposure Route: oral in diet

Dosage: three dose levels:

Calculations:

25,50 , and $100 \mathrm{ppm} ; \mathrm{NOAEL}=100 \mathrm{ppm}$

$$
\left(\frac{100 \mathrm{mg} \text { Lindane }}{\mathrm{kg} \text { food }} \times \frac{28 \mathrm{~g} \text { food }}{\text { day }} \times \frac{1 \mathrm{~kg}}{1000 \mathrm{~g}}\right) / 0.35 \mathrm{~kg} \mathrm{BW}=8 \mathrm{mg} / \mathrm{kg} / \mathrm{d}
$$

Comments: Because significant effects were not observed at any dose level, the 100 ppm was considered to be a chronic NOAEL.

Final NOAEL: $8 \mathrm{mg} / \mathrm{kg} / \mathrm{d}$

\section{Compound: $\quad$ Lindane $(\gamma$-BHC)}

Form: not applicable

Reference: Chakravarty and Lahiri 1986; Chakravarty et al. 1986

Test Species: Mallard Duck

Body weight: $1.0 \mathrm{~kg}$ (Heinz et al. 1989)

Exposure Duration: 8 weeks (during a critical lifestage $=$ chronic).

Endpoint: reproduction

Exposure Route:

Dosage:

oral intubation

one dose level:

$20 \mathrm{mg} / \mathrm{kg} / \mathrm{d}=$ LOAEL

Calculations: not applicable

Comments: Mallards exposed to $20 \mathrm{mg} / \mathrm{kg} / \mathrm{d}$ displayed reduced eggshell thickness, laid fewer eggs and had longer time intervals between eggs. Because the study considered exposure during a critical lifestage, the $20 \mathrm{mg} / \mathrm{kg} / \mathrm{d}$ was considered to be a chronic LOAEL. A chronic NOAEL was estimated by multiplying the chronic LOAEL by a LOAEL-NOAEL uncertainty factor of 0.1 .

Final NOAEL: $2 \mathrm{mg} / \mathrm{kg} / \mathrm{d}$ 
Compound: Lithium

Form: Lithium Carbonate $(18.78 \% \mathrm{Li})$

Reference: $\quad$ Marathe and Thomas 1986

Test Species: Rat

Body weight: $0.35 \mathrm{~kg}$ (EPA 1988a)

Food Consumption: $0.028 \mathrm{~kg} / \mathrm{d}$ (calculated using allometric equation from EPA 1988a)

Exposure Duration: days 6-15 of gestation (during a critical lifestage $=$ chronic).

Endpoint:

Exposure Route:

Dosage: reproduction

oral in diet

two dose levels:

50 and $100 \mathrm{mg} / \mathrm{kg} / \mathrm{d}$ Lithium Carbonate: NOAEL $=50 \mathrm{mg} / \mathrm{kg} / \mathrm{d}$

Calculations: $\quad \mathrm{mg} \mathrm{Li} / \mathrm{kg} / \mathrm{d}=0.1878 \times 50 \mathrm{mg} / \mathrm{kg} / \mathrm{d}=9.39$

Comments: Lithium carbonate exposure of $100 \mathrm{mg} / \mathrm{kg} / \mathrm{d}$ reduced the number of offspring and offspring weights. No adverse effects were observed at the $50 \mathrm{mg} / \mathrm{kg}$ level. While the Lithium exposures evaluated in this study were of a short duration, they occurred during a critical lifestage. Therefore, the $50 \mathrm{mg} / \mathrm{kg} / \mathrm{d}$ dose was considered to be a chronic NOAEL.

Final NOAEL: $9.39 \mathrm{mg} / \mathrm{kg} / \mathrm{d}$

Compound: Manganese

Form: Manganese Oxide $\left(\mathrm{Mn}_{3} \mathrm{O}_{4}\right)$

Reference: Laskey et al. 1982

Test Species: Rat

Body weight: $0.35 \mathrm{~kg}$ (EPA 1988a)

Food Consumption: $0.028 \mathrm{~kg} / \mathrm{d}$ (calculated using allometric equation from EPA 1988a)

Exposure Duration: through gestation for $224 \mathrm{~d}$

Endpoint: (during a critical lifestage $=$ chronic).

Exposure Route: reproduction

Dosage: oral in diet three dose levels: 350,1050 , and $3500 \mathrm{ppm}$ supplemented $\mathrm{Mn}+50 \mathrm{ppm} \mathrm{Mn}$ in base diet; NOAEL $=1100 \mathrm{ppm}$

Calculations:

$$
\left(\frac{1100 \mathrm{mg} \mathrm{Mn}}{\mathrm{kg} \text { food }} \times \frac{28 \mathrm{~g} \text { food }}{\text { day }} \times \frac{1 \mathrm{~kg}}{1000 \mathrm{~g}}\right) / 0.35 \mathrm{~kg} \mathrm{BW}=88 \mathrm{mg} / \mathrm{kg} / \mathrm{d}
$$

Comments: While the pregnancy percentage and fertility among rats consuming $3550 \mathrm{ppm} \mathrm{Mn}$ in their diet was significantly reduced, all other reproductive parameters (e.g., litter size, ovulations, resorptions, preimplantation death, fetal weights) were not affected. No effects were observed at lower Mn exposure levels. Therefore the $1100 \mathrm{ppm} \mathrm{Mn}$ dose 
was considered to be a chronic NOAEL.

Final NOAEL: $88 \mathrm{mg} / \mathrm{kg} / \mathrm{d}$

$\begin{array}{lc}\text { Compound: } & \text { Mercury } \\ \text { Form: } & \text { Mercuric chloride } \\ \text { Reference: } & \text { Knoflach et al, } 1986 \\ \text { Test Species: } & \text { Rat }\end{array}$

Body weight: $0.03 \mathrm{~kg}$ (EPA 1988a)

Exposure Duration: 39 week

Endpoint:

( $<1$ yr and not during a critical lifestage = subchronic).

Exposure Route:

Immune system and kidney impairment

Dosage:

oral intubation

one dose level:

Calculations:

$0.64 \mathrm{mg} / \mathrm{kg} / \mathrm{d}=$ LOAEL not applicable

Comments: Because immune system and kidney function were impaired by the 0.64 $\mathrm{mg} / \mathrm{kg} / \mathrm{d}$ dose level and the study was less than one year in duration and did not consider exposure during critical lifestages, this dose was considered to be a subchronic LOAEL. A chronic NOAEL was estimated by multiplying the subchronic LOAEL by a LOAEL-NOAEL uncertainty factor of 0.1 and a subchronic-chronic uncertainty factor of 0.1 .

Final NOAEL: $0.0064 \mathrm{mg} / \mathrm{kg} / \mathrm{d}$

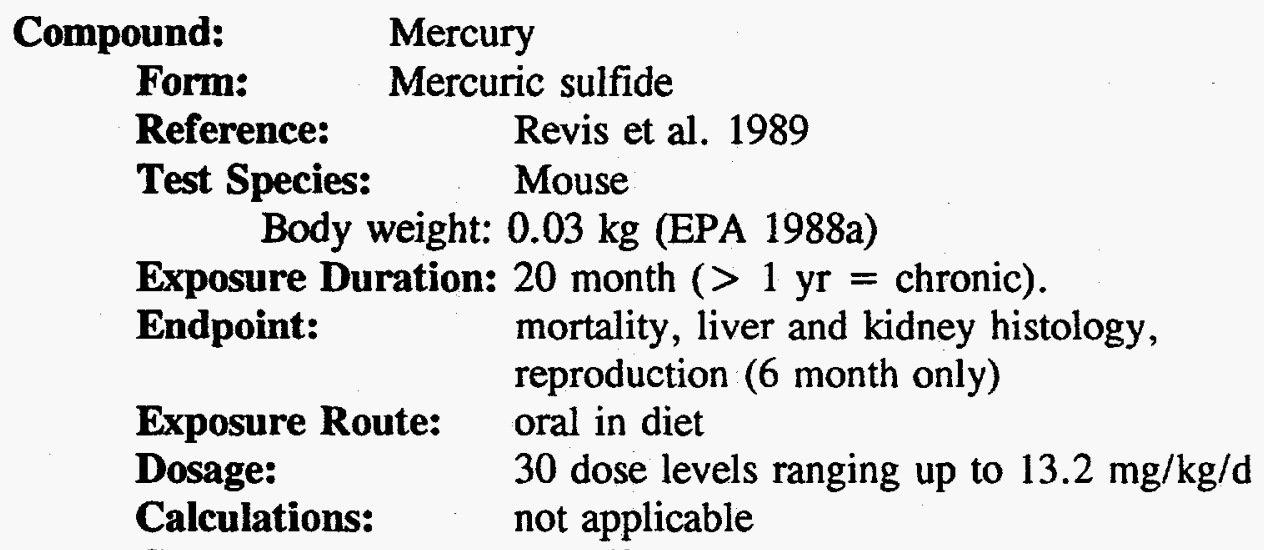

Comments: No adverse effects were observed at any dose level. Because the study was over one year in duration, the maximum dose $13.2 \mathrm{mg} / \mathrm{kg} / \mathrm{d}$ was considered to be a chronic NOAEL.

Final NOAEL: $13.2 \mathrm{mg} / \mathrm{kg} / \mathrm{d}$

$\begin{array}{ll}\text { Compound: } & \text { Mercury } \\ \text { Form: } & \text { Methyl Mercury Chloride } \\ \text { Reference: } & \text { Wobeser et al. } 1976\end{array}$


Test Species: Mink

Body weight: $1 \mathrm{~kg}$ (EPA 1993e)

Food Consumption: $0.137 \mathrm{~kg} / \mathrm{d}$ (Bleavins and Aulerich 1981)

Exposure Duration: 93 days

Endpoint:

Exposure Route:

Dosage:

( $<1 \mathrm{yr}$ and not during a critical lifestage $=$ subchronic). mortality, weight loss, ataxia

oral in diet

five dose levels:

$1.1,1.8,4.8,8.3$, and $15 \mathrm{ppm} \mathrm{Hg}$ as methyl mercury;

NOAEL $=1.1 \mathrm{ppm} \mathrm{Hg}$

Calculations:

$$
\left(\frac{1.1 \mathrm{mg} \mathrm{Hg}}{\mathrm{kg} \text { food }} \times \frac{137 \mathrm{~g} \text { food }}{\text { day }} \times \frac{1 \mathrm{~kg}}{1000 \mathrm{~g}}\right) / 1 \mathrm{~kg} \mathrm{BW}=0.15 \mathrm{mg} / \mathrm{kg} / \mathrm{d}
$$

Comments: Mercury doses of $1.8 \mathrm{ppm}$ or greater produced significant adverse effects (mortality, weight loss, behavioral abnormalities). Because significant effects were not observed at the $1.1 \mathrm{ppm} \mathrm{Hg}$ dose level, this dose was considered to be a subchronic NOAEL. A chronic NOAEL was estimated by multiplying the subchronic NOAEL by a subchronic-chronic uncertainty factor of 0.1

Final NOAEL: $0.015 \mathrm{mg} / \mathrm{kg} / \mathrm{d}$

\section{Compound: Mercury}

Form: $\quad$ Methyl Mercury Chloride $\left(\mathrm{CH}_{3} \mathrm{HgCl} ; 79.89 \% \mathrm{Hg}\right)$

Reference: Verschuuren et al. 1976

Test Species: Rat

Body weight: $0.35 \mathrm{~kg}$ (EPA 1988a)

Food Consumption: $0.028 \mathrm{~kg} / \mathrm{d}$ (calculated using allometric equation from EPA 1988a)

Exposure Duration: 3 generations $(>1$ yr and during a critical lifestage $=$ chronic).

Endpoint:

Exposure Route:

Dosage:

Calculations:

$$
\left(\frac{0.399 \mathrm{mg} \mathrm{Hg}}{\mathrm{kg} \mathrm{food}} \times \frac{28 g \text { food }}{\text { day }} \times \frac{1 \mathrm{~kg}}{1000 \mathrm{~g}}\right) / 0.35 \mathrm{~kg} \mathrm{BW}=0.032 \mathrm{mg} / \mathrm{kg} / \mathrm{d}
$$

Comments: While exposure to $2.5 \mathrm{ppm}$ methyl mercury chloride reduced pup viability, adverse effects were not observed at lower doses. Because significant effects were 
not observed at the $0.5 \mathrm{ppm}$ Methyl Mercury Chloride dose level, this dose was considered to be a chronic NOAEL.

Final NOAEL: $0.032 \mathrm{mg} / \mathrm{kg} / \mathrm{d}$

$\begin{array}{cc}\text { Compound: } & \text { Mercury } \\ \text { Form: } & \text { Methyl Mercury Dicyandiamide } \\ \text { Reference: } & \text { Heinz 1979 } \\ \text { Test Species: } & \text { Mallard Duck }\end{array}$

Body weight: $1 \mathrm{~kg}$ (Heinz et al. 1989)

Food Consumption: $0.128 \mathrm{~kg} / \mathrm{d}$ (from study)

Exposure Duration: 3 generations $(>1 \mathrm{yr}$ and during a critical lifestage $=$ chronic).

Endpoint: reproduction

Exposure Route: oral in diet

Dosage: one dose level:

$0.5 \mathrm{ppm} \mathrm{Hg}$ as Methyl Mercury Dicyandiamide

Calculations:

$$
\text { LOAEL }=0.5 \mathrm{ppm}
$$

$$
\left(\frac{0.5 \mathrm{mg} \mathrm{Hg}}{\mathrm{kg} \mathrm{food}} \times \frac{128 \mathrm{~g} \mathrm{food}}{\text { day }} \times \frac{1 \mathrm{~kg}}{1000 \mathrm{~g}}\right) / 1 \mathrm{~kg} \mathrm{BW}=0.064 \mathrm{mg} / \mathrm{kg} / \mathrm{d}
$$

Comments: Because significant effects (fewer eggs and ducklings were produced) were observed at the $0.5 \mathrm{ppm} \mathrm{Hg}$ dose level and the study consider exposure over three generations, this dose was considered to be a chronic LOAEL. A chronic NOAEL was estimated by multiplying the chronic LOAEL by a LOAEL-NOAEL uncertainty factor of 0.1 .

Final NOAEL: $0.0064 \mathrm{mg} / \mathrm{kg} / \mathrm{d}$

Compound: Methanol

Form: not applicable

Reference: $\quad$ EPA 1986e

Test Species: Rat

Body weight: $0.35 \mathrm{~kg}$ (EPA 1988a)

Exposure Duration: 90 days $(<1$ yr and not during a critical lifestage $=$ subchronic).

Endpoint: mortality, blood chemistry

Exposure Route: oral intubation

Dosage: three dose levels: 100,500 , and $2500 \mathrm{mg} / \mathrm{kg} / \mathrm{d} ; \mathrm{NOAEL}=500 \mathrm{mg} / \mathrm{kg} / \mathrm{d}$

Calculations: not applicable

Comments: While Methanol at $2500 \mathrm{mg} / \mathrm{kg} / \mathrm{d}$ reduced brain and liver weights and altered blood chemistry, no effects were observed at the $500 \mathrm{mg} / \mathrm{kg} / \mathrm{d}$ dose level. Because the study was 90 days in duration and did not consider exposure during critical lifestages, the 
$500 \mathrm{mg} / \mathrm{kg} / \mathrm{d}$ dose was considered to be a chronic LOAEL. A chronic NOAEL was estimated by multiplying the chronic LOAEL by a LOAEL-NOAEL uncertainty factor of 0.1 .

Final NOAEL: $50 \mathrm{mg} / \mathrm{kg} / \mathrm{d}$

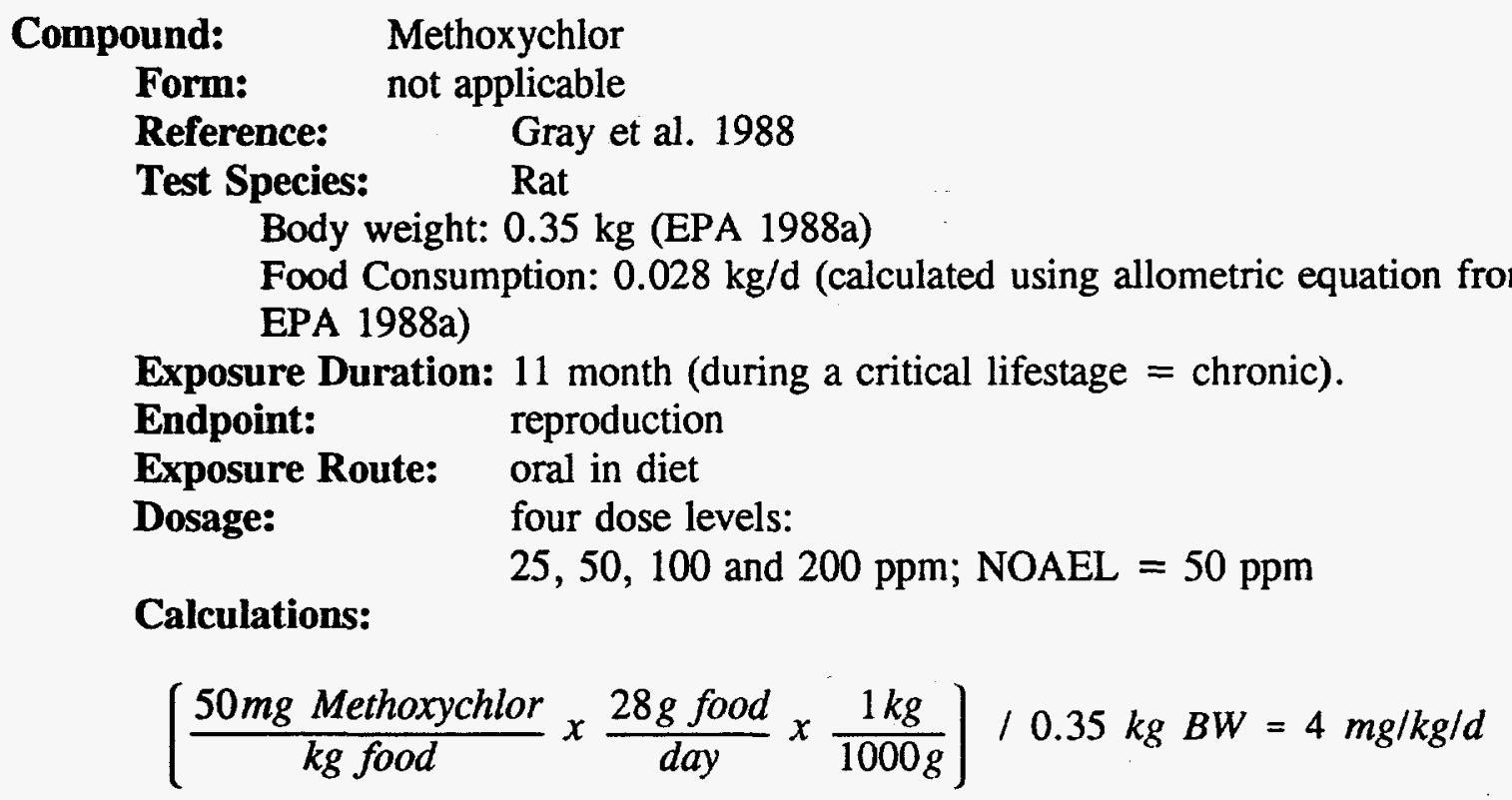

Comments: Fertility and litter size was significantly reduced among rats fed diets containing 100 or $200 \mathrm{ppm}$ methoxychlor. Because significant effects were not observed at the $50 \mathrm{ppm}$ dose level and the study considered exposure during reproduction, the $50 \mathrm{ppm}$ was considered to be a chronic NOAEL.

Final NOAEL: $4 \mathrm{mg} / \mathrm{kg} / \mathrm{d}$

Compound: Methylene Chloride

Form: not applicable

Reference: NCA 1982

Test Species: Rat

Body weight: $0.35 \mathrm{~kg}$ (EPA 1988a)

Exposure Duration: 2 yrs $(>1 \mathrm{yr}=$ chronic).

Endpoint: liver histology

Exposure Route: oral in water

Dosage: four dose levels:

$5.85,50,125$, and $250 \mathrm{mg} / \mathrm{kg} / \mathrm{d} ; \mathrm{NOAEL}=5 \mathrm{mg} / \mathrm{kg} / \mathrm{d}$

Calculations: not applicable

Comments: While Methylene Chloride at $50 \mathrm{mg} / \mathrm{kg} / \mathrm{d}$ or greater produced histological changes in the liver, no effects were observed at the $5.85 \mathrm{mg} / \mathrm{kg} / \mathrm{d}$ dose level. 
Because the study was 2 yrs in duration, the $5.85 \mathrm{mg} / \mathrm{kg} / \mathrm{d}$ dose was considered to be a chronic NOAEL.

Final NOAEL: $5.85 \mathrm{mg} / \mathrm{kg} / \mathrm{d}$

\author{
Compound: $\quad$ Methyl Ethyl Ketone \\ Form: not applicable \\ Reference: $\quad$ Cox et al. 1975 \\ Test Species: Rat \\ Body weight: $0.35 \mathrm{~kg}$ (EPA 1988a) \\ Exposure Duration: 2 generations ( $>1 \mathrm{yr}$ and during a critical lifestage $=$ chronic). \\ Endpoint: \\ reproduction \\ Exposure Route: oral in water \\ Dosage: \\ three dose levels: \\ 538,1644 , and $5089 \mathrm{mg} / \mathrm{kg} / \mathrm{d}$ (males), \\ 594,1771 , and $4571 \mathrm{mg} / \mathrm{kg} / \mathrm{d}$ (females); \\ NOAEL $=1771 \mathrm{mg} / \mathrm{kg} / \mathrm{d}$ \\ Calculations: \\ not applicable \\ Comments: While Methyl Ethyl Ketone at the highest dose levels reduced the \\ number of pups/litter, pup survivorship, and pup body weight, no adverse effects were \\ observed at the next higher levels $(1644 \mathrm{mg} / \mathrm{kg} / \mathrm{d}$ and $1771 \mathrm{mg} / \mathrm{kg} / \mathrm{d}$ for males and females \\ respectively). Because the study was 2 generations in duration, the $1771 \mathrm{mg} / \mathrm{kg} / \mathrm{d}$ dose was \\ considered to be a chronic NOAEL.
}

Final NOAEL: $1771 \mathrm{mg} / \mathrm{kg} / \mathrm{d}$

\section{Compound:}

Form:

Reference:

Test Species:
4-Methyl 2-Pentanone (Methyl Isobutyl Ketone) not applicable

Microbiological Associates 1986 (obtained from Health Effects Assessment Summary Tables (HEAST; EPA 1993f)

Body weight: $0.35 \mathrm{~kg}$ (EPA 1988a)

Exposure Duration: 13 weeks

( $<1 \mathrm{yr}$ and not during a critical lifestage $=$ subchronic).

Endpoint:

Exposure Route:

Dosage:

Liver and kidney function

oral gavage

one dose level stated in HEAST summary:

$250 \mathrm{mg} / \mathrm{kg} / \mathrm{d}=$ NOAEL

Calculations: not applicable

Comments: Because the study was less than 1 year in duration and not considered exposure during a critical life stage, the $250 \mathrm{mg} / \mathrm{kg} / \mathrm{d}$ dose was considered to be a subchronic NOAEL. A chronic NOAEL was estimated by multiplying the subchronic NOAEL by a subchronic-chronic uncertainty factor of 0.1

Final NOAEL: $25 \mathrm{mg} / \mathrm{kg} / \mathrm{d}$ 
Compound: Nickel

Form: Nickel Sulfate Hexahydrate

Reference: $\quad$ Ambrose et al. 1976

Test Species: Rat

Body weight: $0.35 \mathrm{~kg}$ (EPA 1988a)

Food Consumption: $0.028 \mathrm{~kg} / \mathrm{d}$ (calculated using allometric equation from EPA 1988a)

Exposure Duration: 3 generations $(>1$ yr and during a critical lifestage $=$ chronic).

Endpoint:

Exposure Route: reproduction

Dosage:

oral in diet

three dose levels:

250,500 , and $1000 \mathrm{ppm} \mathrm{Ni}$

$\mathrm{NOAEL}=500 \mathrm{ppm}$

\section{Calculations:}

$$
\left(\frac{500 \mathrm{mg} \mathrm{Ni}}{\mathrm{kg} \mathrm{food}} \times \frac{28 \mathrm{~g} \text { food }}{\text { day }} \times \frac{1 \mathrm{~kg}}{1000 \mathrm{~g}}\right] / 0.35 \mathrm{~kg} \mathrm{BW}=40 \mathrm{mg} / \mathrm{kg} / \mathrm{d}
$$

Comments: While $1000 \mathrm{ppm} \mathrm{Ni}$ in the diet reduced offspring body weights, no adverse effects were observed in the other dose levels. Because this study considers exposures over multiple generations, the $500 \mathrm{ppm}$ dose was considered to be a chronic NOAEL.

Final NOAEL: $40 \mathrm{mg} / \mathrm{kg} / \mathrm{d}$

\section{Compound: Nickel \\ Form: Nickel Sulfate \\ Reference: Cain and Pafford 1981 \\ Test Species: Mallard Duckling}

Body weight: $0.782 \mathrm{~kg}$ (mean control $\delta+q_{\text {at }}$ at 45 days; from study )

Food Consumption: Adult Mallard ducks, weighing $1 \mathrm{~kg}$ consume

$100 \mathrm{~g}$ food/d (Heinz et al.1989). Therefore, it was assumed that a

$0.782 \mathrm{~kg}$ mallard duckling would consume $78.2 \mathrm{~g}$ food $/ \mathrm{d}$.

Exposure Duration: $90 \mathrm{~d}$ ( $>10$ week = chronic).

Endpoint:

Exposure Route:

Dosage: mortality, growth, behavior

oral in diet

three dose levels:

176, 774, and $1069 \mathrm{ppm} \mathrm{Ni}$;

NOAEL $=774 \mathrm{ppm}$ 
Calculations:

$$
\left(\frac{774 \mathrm{mg} \mathrm{Ni}}{\mathrm{kg} \text { food }} \times \frac{78.2 \mathrm{~g} \text { food }}{\text { day }} \times \frac{1 \mathrm{~kg}}{1000 \mathrm{~g}}\right) / 0.782 \mathrm{~kg} \mathrm{BW}=77.4 \mathrm{mg} / \mathrm{kg} / \mathrm{d}
$$

Comments: Consumption of up to $774 \mathrm{ppm} \mathrm{Ni}$ in diet did not increase mortality or reduce growth. Because the study considered exposure over 90 days, the $774 \mathrm{ppm}$ dose was considered to be a chronic NOAEL To estimate daily Ni intake throughout the 90 day study period, food consumption of 45-day-old ducklings was calculated. While this value will over- and underestimate food consumption by younger and older ducklings, it was assumed to approximate food consumption throughout the entire 90 day study.

Final NOAEL: $77.4 \mathrm{mg} / \mathrm{kg} / \mathrm{d}$

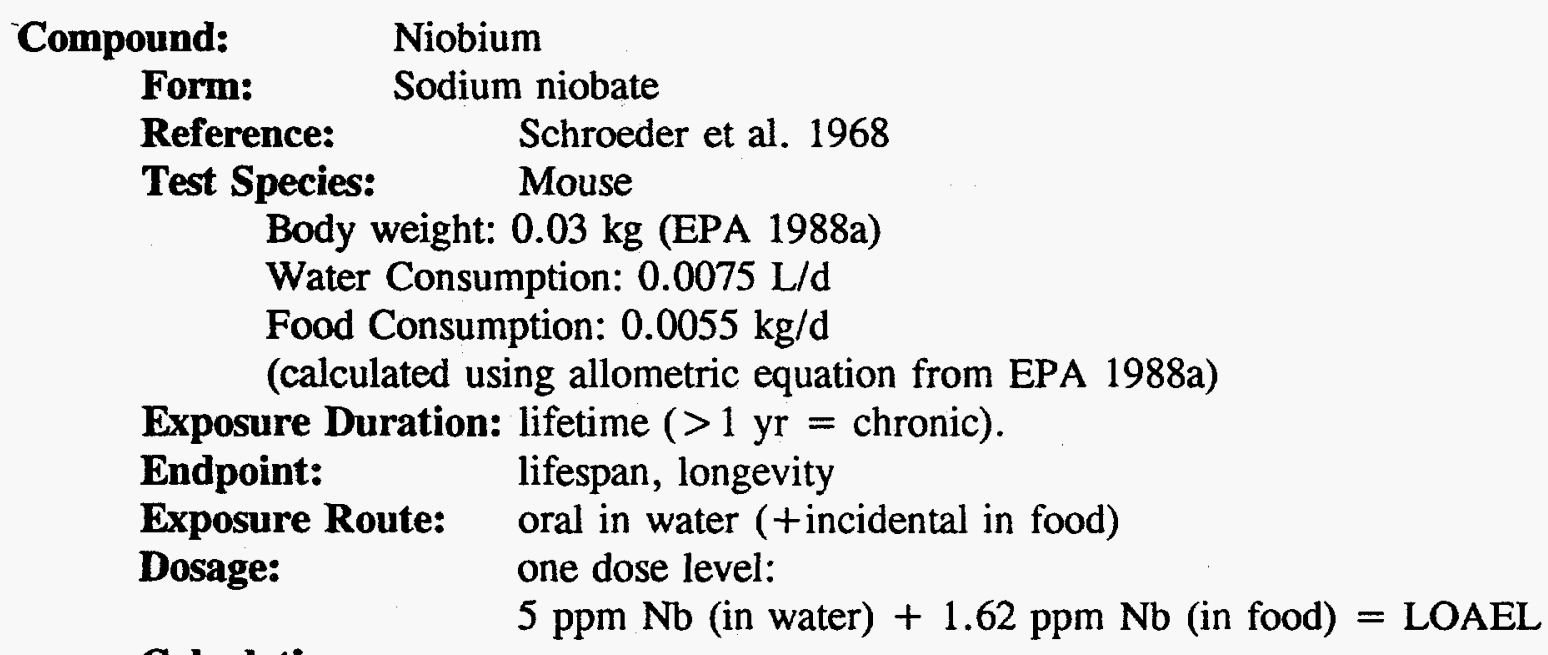

\section{Calculations:}

$$
\begin{aligned}
& \left(\frac{5 \mathrm{mg} \mathrm{Nb}}{L \text { water }} \times \frac{7.5 \mathrm{~mL} \text { water }}{\text { day }} \times \frac{1 \mathrm{~L}}{1000 \mathrm{~mL}}\right) / 0.03 \mathrm{~kg} \mathrm{BW}=1.25 \mathrm{mg} / \mathrm{kg} / \mathrm{d} \\
& {\left[\frac{1.62 \mathrm{mg} \mathrm{Nb}}{\mathrm{kg} \mathrm{food}} \times \frac{5.5 g \text { food }}{\text { day }} \times \frac{1 \mathrm{~kg}}{1000 \mathrm{~g}}\right] / 0.03 \mathrm{~kg} \mathrm{BW}=0.297 \mathrm{mg} / \mathrm{kg} / \mathrm{d}} \\
& \text { Total Exposure }=1.25 \mathrm{mg} / \mathrm{kg} / \mathrm{d}+0.297 \mathrm{mg} / \mathrm{kg} / \mathrm{d}=1.547 \mathrm{mg} / \mathrm{kg} / \mathrm{d}
\end{aligned}
$$

Comments: Because median lifespan was reduced among female mice exposed to the $5 \mathrm{ppm}$ dose level and the study considered exposure throughout the entire lifespan, this dose was considered to be a chronic LOAEL. A chronic NOAEL was estimated by multiplying the chronic LOAEL by a LOAEL-NOAEL uncertainty factor of 0.1 .

Final NOAEL: $0.1166 \mathrm{mg} / \mathrm{kg} / \mathrm{d}$ 


\section{Compound: Nitrate}
Form: Potassium Nitrate
Reference: $\quad$ Sleight and Atallah 1968
Test Species: Guinea pig
Body weight: $0.86 \mathrm{~kg}$ (EPA 1988a)
Exposure Duration: 143-204 days (during a critical lifestage=chronic).
Endpoint: reproduction
Exposure Route: oral in water
Dosage:
four dose levels:
$12,102,507$, and $1130 \mathrm{mg}$ nitrate-Nitrogen $\mathrm{kg} / \mathrm{d}$;
$\mathrm{NOAEL}=507 \mathrm{mg} / \mathrm{kg} / \mathrm{d}$
Calculations: not applicable

Comments: While Nitrate at the highest dose level reduced the number of live births, no adverse effects were observed at the other dose levels. Because the study considered exposure during reproduction, the $507 \mathrm{mg} / \mathrm{kg} / \mathrm{d}$ dose was considered to be a chronic NOAEL.

Final NOAEL: $507 \mathrm{mg} / \mathrm{kg} / \mathrm{d}$

\section{Compound: $\quad 1,2,3,4,8$ - Pentachloro Dibenzofuran (PeDBF) \\ Form: not applicable}

Reference: $\quad$ Poiger et al. 1989

Test Species: Rat

Body weight: $0.35 \mathrm{~kg}$ (EPA 1988a)

Food Consumption: $0.028 \mathrm{~kg} / \mathrm{d}$ (calculated using allometric equation from EPA 1988a)

Exposure Duration: 13 weeks

( $<1 \mathrm{yr}$ and not during a critical lifestage $=$ subchronic).

Endpoint:

Exposure Route:

Body weight, organ weight, blood chemistry

Dosage:

oral in diet

two dose levels:

600 and $6000 \mathrm{ppb} ;$ NOAEL $=6000 \mathrm{ppb}$

\section{Calculations:}

$$
\left(\frac{6 \mathrm{mg} P e D B F}{\mathrm{~kg} \text { food }} \times \frac{28 \mathrm{~g} \text { food }}{\text { day }} \times \frac{1 \mathrm{~kg}}{1000 \mathrm{~g}}\right) / 0.35 \mathrm{~kg} \mathrm{BW}=0.48 \mathrm{mg} / \mathrm{kg} / \mathrm{d}
$$

Comments: Because no significant effects were observed at either dose level, the $6000 \mathrm{ppb}$ dose was considered to be a subchronic NOAEL. A chronic NOAEL was estimated by multiplying the subchronic NOAEL by a subchronic-chronic uncertainty factor of 0.1 .

Final NOAEL: $0.048 \mathrm{mg} / \mathrm{kg} / \mathrm{d}$ 
Compound: $\quad 1,2,3,7,8$ - Pentachloro Dibenzofuran (PeDBF)

Form: not applicable

Reference: $\quad$ Poiger et al. 1989

Test Species: Rat

Body weight: $0.35 \mathrm{~kg}$ (EPA 1988a)

Food Consumption: $0.028 \mathrm{~kg} / \mathrm{d}$ (calculated using allometric equation from EPA 1988a)

Exposure Duration: 13 weeks

Endpoint:

Exposure Route:

Dosage:

( $<1 \mathrm{yr}$ and not during a critical lifestage $=$ subchronic $)$.

Calculations:

Body weight, organ weight, blood chemistry

oral in diet

three dose levels:

2,20 , and $200 \mathrm{ppb} ;$ NOAEL $=20 \mathrm{ppb}$

$$
\left(\frac{0.02 \mathrm{mg} \mathrm{HxDBF}}{\mathrm{kg} \text { food }} \times \frac{28 \mathrm{~g} \text { food }}{\text { day }} \times \frac{1 \mathrm{~kg}}{1000 \mathrm{~g}}\right) / 0.35 \mathrm{~kg} \mathrm{BW}=0.0016 \mathrm{mg} / \mathrm{kg} / \mathrm{d}
$$

Comments: Because rats exposed to $200 \mathrm{ppb}$ PeDBF in the diet displayed reduced body, thymus weights, while those in the $20 \mathrm{ppb}$ group did not, the $20 \mathrm{ppb}$ dose was considered to be a subchronic NOAEL. A chronic NOAEL was estimated by multiplying the subchronic NOAEL by a subchronic-chronic uncertainty factor of 0.1 .

Final NOAEL: $0.00016 \mathrm{mg} / \mathrm{kg} / \mathrm{d}$

Compound: $\quad 2,3,4,7,8$ - Pentachloro Dibenzofuran (PeDBF)

Form: not applicable

Reference: $\quad$ Poiger et al. 1989

Test Species: Rat

Body weight: $0.35 \mathrm{~kg}$ (EPA 1988a)

Food Consumption: $0.028 \mathrm{~kg} / \mathrm{d}$ (calculated using allometric equation from EPA 1988a)

Exposure Duration: 13 weeks

Endpoint: $(<1 \mathrm{yr}$ and not during a critical lifestage $=$ subchronic $)$.

Exposure Route:

Dosage:

Body weight, organ weight, blood chemistry

oral in diet

three dose levels:

Calculations:

2,20 , and $200 \mathrm{ppb} ; \mathrm{NOAEL}=2 \mathrm{ppb}$

$\left(\frac{0.002 \mathrm{mg} \mathrm{PeDBF}}{\mathrm{kg} \mathrm{food}} \times \frac{28 \mathrm{~g} \text { food }}{\text { day }} \times \frac{1 \mathrm{~kg}}{1000 \mathrm{~g}}\right) / 0.35 \mathrm{~kg} \mathrm{BW}=0.00016 \mathrm{mg} / \mathrm{kg} / \mathrm{d}$ 
Comments: Because rats exposed to 20 and $200 \mathrm{ppb}$ PeDBF in the diet displayed reduced body, thymus and liver weights, while those in the $2 \mathrm{ppb}$ group did not, the $2 \mathrm{ppb}$ dose was considered to be a subchronic NOAEL. A chronic NOAEL was estimated by multiplying the subchronic NOAEL by a subchronic-chronic uncertainty factor of 0.1 .

Final NOAEL: $0.000016 \mathrm{mg} / \mathrm{kg} / \mathrm{d}$

Compound: Pentachloronitrobenzene (PCNB)

Form: not applicable

Reference: Dunn et al. 1979

Test Species: Chicken

Body weight: $1.5 \mathrm{~kg}$ (EPA 1988a)

Food Consumption: $0.106 \mathrm{~kg} / \mathrm{d}$ (calculated using allometric equation from EPA 1988a)

Exposure Duration: 35 weeks

Endpoint:

$(>10$ weeks and during a critical lifestage $=$ chronic $)$.

Exposure Route: reproduction

Dosage: oral in diet four dose levels:

$10,50,100$, and $1000 \mathrm{ppm} ;$ NOAEL $=100 \mathrm{ppm}$

Calculations:

$$
\left(\frac{100 \mathrm{mg} \mathrm{PCNB}}{\mathrm{kg} \text { food }} \times \frac{106 \mathrm{~g} \text { food }}{d a y} \times \frac{1 \mathrm{~kg}}{1000 \mathrm{~g}}\right) / 1.5 \mathrm{~kg} \mathrm{BW}=7.07 \mathrm{mg} / \mathrm{kg} / \mathrm{d}
$$

Comments: Onset on egg production and egg hatchability was reduced among birds receiving $1000 \mathrm{ppm}$ PCNB. No adverse effects were observed among the other dose levels. Because the study considered exposure through reproduction, the $100 \mathrm{ppm}$ dose was considered to be a chronic NOAEL.

Final NOAEL: $7.07 \mathrm{mg} / \mathrm{kg} / \mathrm{d}$

\section{Compound: Selenium \\ Form: $\quad$ Selanate $\left(\mathrm{SeO}_{4}\right)$ \\ Reference: $\quad$ Schroeder and Mitchner 1971 \\ Test Species: Mouse}

Body weight: $0.03 \mathrm{~kg}$ (EPA 1988a)

Water Consumption: $0.0075 \mathrm{~L} / \mathrm{d}$

(calculated using allometric equation from EPA 1988a)

Exposure Duration: 3 generations ( $>1 \mathrm{yr}$ and during critical lifestage $=$ chronic)

Endpoint: reproduction

Exposure Route: oral in water

Dosage: one dose level:

$3 \mathrm{mg} \mathrm{Se} / \mathrm{L}=$ LOAEL 


\title{
Calculations:
}

$$
\left(\frac{3 \mathrm{mg} \mathrm{Se}}{L \text { water }} \times \frac{7.5 L \text { water }}{\text { day }} \times \frac{1 L}{1000 \mathrm{~mL}}\right) / 0.03 \mathrm{~kg} \mathrm{BW}=0.75 \mathrm{mg} / \mathrm{kg} / \mathrm{d}
$$

Comments: Because mice exposed to Se displayed reduced reproductive success with a high incidence of runts and failure to breed, this dose was considered to be a chronic LOAEL. A chronic NOAEL was estimated by multiplying the chronic LOAEL by a LOAEL-NOAEL uncertainty factor of 0.1 .

Final NOAEL: $0.075 \mathrm{mg} / \mathrm{kg} / \mathrm{d}$

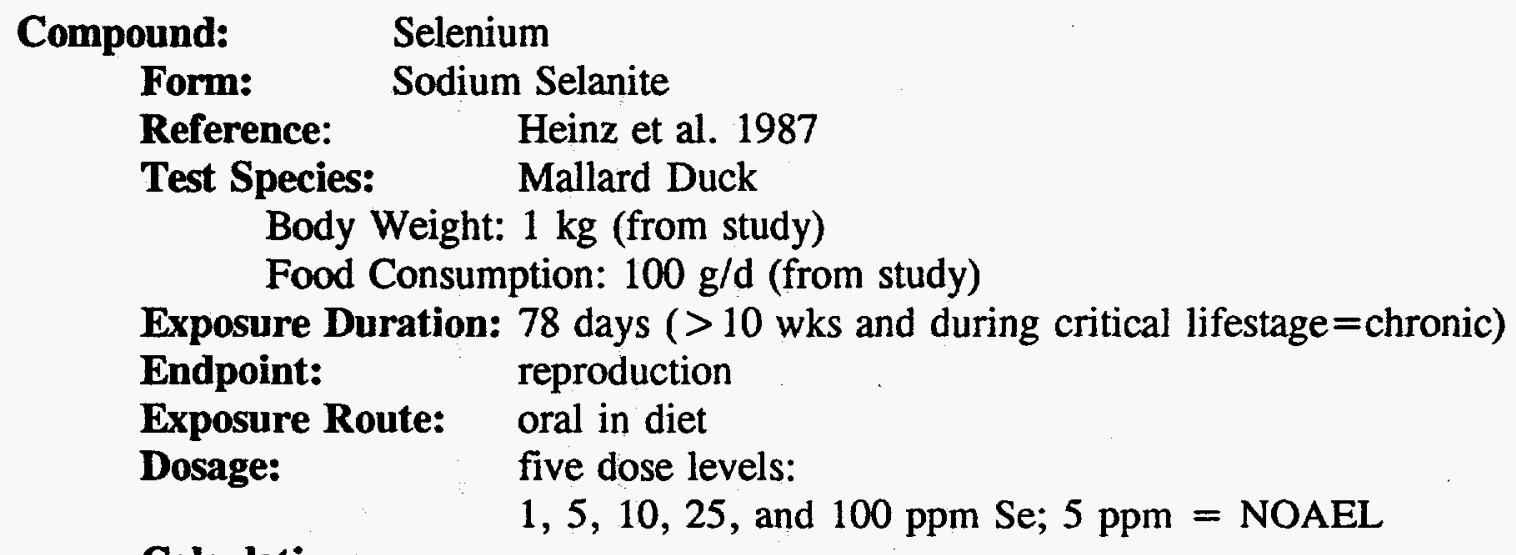

Calculations:

$$
\left(\frac{5 \mathrm{mg} \mathrm{Se}}{\mathrm{kg} \text { food }} \times \frac{100 \mathrm{~g} \text { food }}{\text { day }} \times \frac{1 \mathrm{~kg}}{1000 \mathrm{mg}}\right) / 1 \mathrm{~kg} \mathrm{BW}=0.5 \mathrm{mg} / \mathrm{kg} / \mathrm{d}
$$

Comments: While consumption of 1,5 , or $10 \mathrm{ppm}$ Se on the diet as Sodium Selanite had no effect on weight or survival of adults, $100 \mathrm{ppm}$ Se reduced adult survival and $25 \mathrm{ppm}$ Se reduced duckling survival. Consumption of 10 or $25 \mathrm{ppm}$ Se in the diet resulted in a significantly larger frequency of lethally deformed embryos as compared to the 1 or $5 \mathrm{ppm}$ Se exposures. Because $5 \mathrm{ppm}$ Se in the diet was the highest dose level that produced no adverse effects and the study considered exposure through reproduction, this dose was considered to be a chronic NOAEL.

Final NOAEL: $0.5 \mathrm{mg} / \mathrm{kg} / \mathrm{d}$

\author{
Compound: Selenium \\ Form: Selanomethionine \\ Reference: $\quad$ Heinz et al. 1989 \\ Test Species: Mallard Duck \\ Body Weight: $1 \mathrm{~kg}$ (from study) \\ Food Consumption: $100 \mathrm{~g} / \mathrm{d}$ (from study)
}


Exposure Duration: 100 days ( $>10$ wks and during critical lifestage $=$ chronic)

Endpoint:

Exposure Route:

Dosage: reproduction

oral in diet

five dose levels:

$1,2,4,8$, and $16 \mathrm{ppm} \mathrm{Se} ; 5 \mathrm{ppm}=\mathrm{NOAEL}$

Calculations:

$$
\left(\frac{4 \mathrm{mg} \mathrm{Se}}{\mathrm{kg} \text { food }} \times \frac{100 \mathrm{~g} \text { food }}{\text { day }} \times \frac{1 \mathrm{~kg}}{1000 \mathrm{mg}}\right) / 1 \mathrm{~kg} \mathrm{BW}=0.4 \mathrm{mg} / \mathrm{kg} / \mathrm{d}
$$

Comments: Consumption of 8 or $16 \mathrm{ppm} \mathrm{Se}$ in the diet as Selanomethionine resulted in a reduced duckling survival as compared to the 1,2, or $4 \mathrm{ppm}$ Se exposures. Because $4 \mathrm{ppm} \mathrm{Se}$ in the diet was the highest dose level that produced no adverse effects and the study considered exposure through reproduction, this dose was considered to be a chronic NOAEL.

Final NOAEL: $0.4 \mathrm{mg} / \mathrm{kg} / \mathrm{d}$

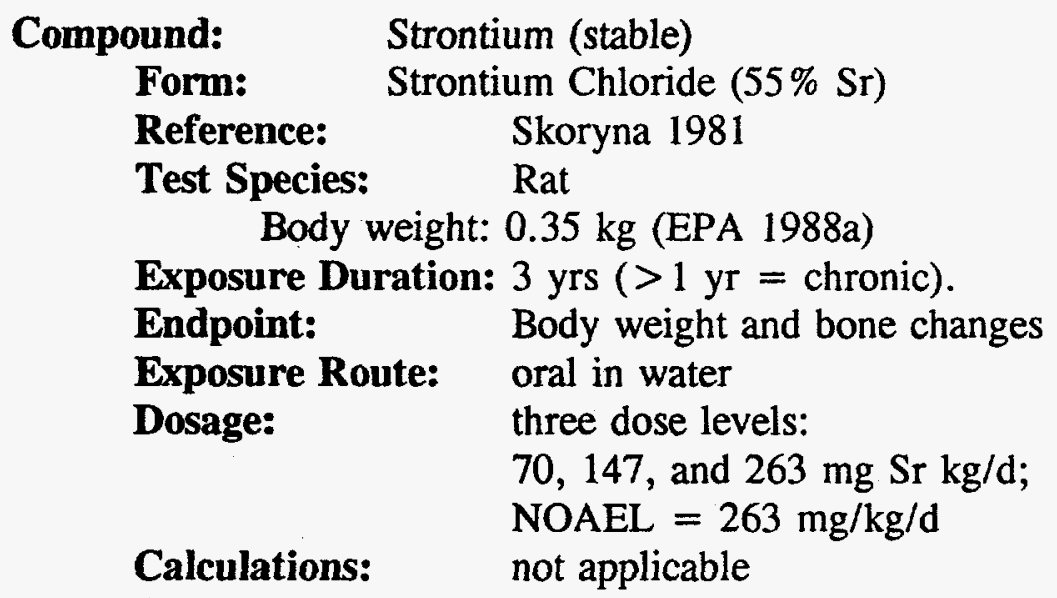

Comments: No adverse effects were observed for any $\mathrm{Sr}$ dosage level. Therefore, because the study considered exposure over three years, the maximum dose was considered to be a chronic NOAEL.

Final NOAEL: $263 \mathrm{mg} / \mathrm{kg} / \mathrm{d}$

Compound: $\quad 2,3,7,8$ - Tetrachloro Dibenzodioxin (TCDD)

Form: not applicable

Reference: $\quad$ Murray et al. 1979

Test Species: Rat

Body weight: $0.35 \mathrm{~kg}$ (EPA 1988a)

Food Consumption: $0.028 \mathrm{~kg} / \mathrm{d}$ (calculated using allometric equation from EPA 1988a)

Exposure Duration: 3 generations $(>1 \mathrm{yr}$ and during a critical lifestage $=$ chronic). 


$\begin{array}{ll}\text { Endpoint: } & \begin{array}{l}\text { reproduction } \\ \text { oral in diet } \\ \text { three dose levels: }\end{array} \\ \text { Dosage: } & 0.001,0.01, \text { and } 0.01 \mathrm{ug} / \mathrm{kg} \mathrm{BW} / \mathrm{d} ; \text { NOAEL }=0.001 \mathrm{ug} / \mathrm{kg} / \mathrm{d} \\ \text { Calculations: } & 0.001 \mathrm{ug} / \mathrm{kg} / \mathrm{d}=0.000001 \mathrm{mg} / \mathrm{kg} / \mathrm{d}\end{array}$

Comments: Fertility and neonatal survival was significantly reduced among rats receiving 0.1 and $0.01 \mathrm{ug} / \mathrm{kg} / \mathrm{d}$. Because no significant differences were observed at the $0.001 \mathrm{ug} / \mathrm{kg} / \mathrm{d}$ dose level and the study considered exposure throughout 3 generations including critical lifestages (reproduction), this dose was considered to be a chronic NOAEL.

Final NOAEL: $0.000001 \mathrm{mg} / \mathrm{kg} / \mathrm{d}$

\section{Compound: $\quad 2,3,7,8$ - Tetrachloro Dibenzodioxin (TCDD) \\ Form: not applicable \\ Reference: $\quad$ Nosek et al. 1992 \\ Test Species: Ring-necked Pheasant}

Body weight: $1 \mathrm{~kg}$ (EPA 1993e)

Exposure Duration: 10 weeks (10 week and during a critical lifestage $=$ chronic).

Endpoint:

reproduction

Exposure Route:

Dosage:

weekly intraperitoneal injection

three dose levels:

$0.01,0.1$, and $1 \mathrm{ug} / \mathrm{kg} \mathrm{BW} /$ week; NOAEL $=0.1 \mathrm{ug} / \mathrm{kg} /$ week

Calculations: $\quad 0.1 \mathrm{ug} / \mathrm{kg} /$ week $=0.0001 \mathrm{mg} / \mathrm{kg} /$ week $=0.000014 \mathrm{mg} / \mathrm{kg} / \mathrm{d}$

Comments: Egg production and hatchability was significantly reduced among birds receiving $1 \mathrm{ug} / \mathrm{kg} /$ week dose. No significant effects were observed among the other two dose levels. The weekly intraperitoneal injection exposure route used in this study is believed to be comparable to oral routes of exposure (EPA 1993e). Because no significant differences were observed at the two lower dose levels and the study considered exposure throughout a critical lifestage (reproduction), the $0.1 \mathrm{ug} / \mathrm{kg} /$ week dose was considered to be a chronic NOAEL.

Final NOAEL: $0.000014 \mathrm{mg} / \mathrm{kg} / \mathrm{d}$

\section{Compound: $\quad 2,3,7,8-$ Tetrachloro Dibenzofuran (TDBF)}

Form: not applicable

Reference: $\quad$ McKinney et al. 1976

Test Species: 1-day old chicks

Body weight: $0.121 \mathrm{~kg}$ (mean $_{\delta+q}$ at $14 \mathrm{~d}$; EPA 1988a)

Food Consumption: $0.0126 \mathrm{~kg} / \mathrm{d}$ (calculated using allometric equation from EPA 1988a)

Exposure Duration: $21 \mathrm{~d}$

Endpoint:

( $<10$ weeks and not during a critical lifestage $=$ subchronic $)$. mortality, weight gain 
Exposure Route: oral in diet

Dosage:

two dose levels:

1 and $5 \mathrm{ppb} ;$ LOAEL $=1 \mathrm{ppb}$

Calculations:

$\left(\frac{0.001 \mathrm{mg} \text { TDBF }}{\mathrm{kg} \text { food }} \times \frac{12.6 \mathrm{~g} \mathrm{food}}{\text { day }} \times \frac{1 \mathrm{~kg}}{1000 \mathrm{~g}}\right) / 0.121 \mathrm{~kg} \mathrm{BW}=0.0001 \mathrm{mg} / \mathrm{kg} / \mathrm{d}$

Comments: Because chicks exposed to 1 and 5 ppb TDBF experienced $16 \%$ and $100 \%$ mortality, respectively, the $1 \mathrm{ppb}$ dose was considered to be a subchronic LOAEL. A chronic NOAEL was estimated by multiplying the subchronic LOAEL by a subchronicchronic uncertainty factor of 0.1 and a LOAEL-NOAEL uncertainty factor of 0.1 . To estimate daily TDBF intake throughout the $21 \mathrm{~d}$ study period, food consumption of 2-weekold chicks was calculated. While this value will over- and underestimate food consumption by younger and older chicks, it was assumed to approximate food consumption throughout the entire 21 day study.

Final NOAEL: $0.000001 \mathrm{mg} / \mathrm{kg} / \mathrm{d}$

Compound: $\quad 1,1,2,2$-Tetrachloroethylene

Form: not applicable

Reference: Buben and O'Flaherty 1985

Test Species: $\quad$ Mouse

Body weight: $0.03 \mathrm{~kg}$ (EPA 1988a)

Exposure Duration: 6 weeks

Endpoint:

$(<1$ yr and not during a critical lifestage $=$ subchronic $)$.

Exposure Route: Hepatotoxicity

Dosage: oral gavage seven dose levels (administered daily 5 days/week for 6 weeks): $20,100,200,500,1000,1500$, and $2000 \mathrm{mg} / \mathrm{kg} / \mathrm{d}$;

NOAEL $=20 \mathrm{mg} / \mathrm{kg} / \mathrm{d}$

Calculations: not applicable

Comments: Because mice were exposed for 5 days/week, 7 day/week exposure were estimated by multiplying doses by 0.7 ( 5 days/7 days). Hepatotoxicity was observed at doses of $100 \mathrm{mg} / \mathrm{kg} / \mathrm{d}$ or greater. Therefore, the $20 \mathrm{mg} / \mathrm{kg} / \mathrm{d}$ dose was considered to be a subchronic NOAEL. A chronic NOAEL was estimated by multiplying the subchronic NOAEL by a subchronic-chronic uncertainty factor of 0.1

Final NOAEL: $1.4 \mathrm{mg} / \mathrm{kg} / \mathrm{d}$

Compound: Thallium

Form: Thallium Sulfate

Reference: $\quad$ Formigli et al. 1986 
Test Species: Rat

Body weight: $0.365 \mathrm{~kg}$ (from study)

Exposure Duration: 60 days

Endpoint:

Exposure Route:

Dosage:

Calculations:
( $<1 \mathrm{yr}$ and not during a critical lifestage $=$ subchronic). reproduction (male testicular function) oral in water one dose level: $10 \mathrm{ppm} \mathrm{Tl}=$ LOAEL mean daily intake (from study) $=270 \mathrm{ug} \mathrm{Tl} / \mathrm{rat}$ $=0.74 \mathrm{mg} / \mathrm{kg} / \mathrm{d}$

Comments: Because rats exposed to $10 \mathrm{ppm} \mathrm{Tl}$ in the diet displayed reduced sperm motility and the study considered exposures only for $60 \mathrm{~d}$, this dose was considered to be a subchronic LOAEL. A chronic NOAEL was estimated by multiplying the subchronic LOAEL by a subchronic-chronic uncertainty factor of 0.1 and a LOAEL-NOAEL uncertainty factor of 0.1 .

Final NOAEL: $0.0074 \mathrm{mg} / \mathrm{kg} / \mathrm{d}$

\begin{tabular}{|c|c|c|}
\hline $\begin{array}{l}\text { Compound: } \\
\text { Form: }\end{array}$ & plicable & \\
\hline Reference: & Nawrot and Staples 1979 & \\
\hline Test Species: & Mouse & \\
\hline Body weight: & $0.03 \mathrm{~kg}$ (EPA 1988a) & \\
\hline Exposure Duration: & days $6-12$ of gestation & \\
\hline & (during a critical lifestage $=$ chro & hic). \\
\hline Endpoint: & reproduction & \\
\hline Exposure Route: & oral gavage & \\
\hline & $\begin{array}{l}\text { three dose levels: } \\
0.3 .0 .5 \text { and } 1 \mathrm{~mL} / \mathrm{kg} / \mathrm{d}: \text { LOAEL }\end{array}$ & $=0.3 \mathrm{~mL} / \mathrm{kg} / \mathrm{d}$ \\
\hline Calculations: & density of toluene $=0.866 \mathrm{~g} / \mathrm{mL}$ & Merck 1976) \\
\hline$\frac{0.3 \mathrm{~mL} \text { Toluer }}{\mathrm{kg} \mathrm{BW}}$ & ne $\times \frac{0.866 \mathrm{~g} \text { Toluene }}{\mathrm{mL} \text { Toluene }} \times \frac{1000 \mathrm{mg}}{1 \mathrm{~g}}$ & $259.8 m$ \\
\hline
\end{tabular}

Comments: Toluene exposure of 0.5 and $1.0 \mathrm{~mL} / \mathrm{kg} / \mathrm{d}$ significantly reduced fetal weights. Embryomortality was significantly reduced by all three dose levels. While the toluene exposures evaluated in this study were of a short duration, they occurred during a critical lifestage. Therefore, the $0.3 \mathrm{~mL} / \mathrm{kg} / \mathrm{d}$ dose was considered to be a chronic LOAEL. A chronic NOAEL was estimated by multiplying the chronic LOAEL by a LOAEL-NOAEL uncertainty factor of 0.1 .

Final NOAEL: $25.98 \mathrm{mg} / \mathrm{kg} / \mathrm{d}$

$\begin{aligned} \text { Compound: } & \text { Toxaphene } \\ \text { Form: } & \text { not applicable }\end{aligned}$


Reference: $\quad$ Kennedy et al. 1973

Test Species: Rat

Body weight: $0.35 \mathrm{~kg}$ (EPA 1988a)

Food Consumption: $0.028 \mathrm{~kg} / \mathrm{d}$ (calculated using allometric equation from EPA 1988a)

Exposure Duration: 3 generations $(>1$ yr and during a critical lifestage $=$ chronic).

Endpoint:

Exposure Route:

Dosage:

Calculations: reproduction

oral in diet

two dose levels:

25 and $100 \mathrm{ppm} ; \mathrm{NOAEL}=100 \mathrm{ppm}$

$$
\left(\frac{100 \mathrm{mg} \text { Toxaphene }}{\mathrm{kg} \mathrm{food}} \times \frac{28 \mathrm{~g} \text { food }}{\text { day }} \times \frac{1 \mathrm{~kg}}{1000 \mathrm{~g}}\right) / 0.35 \mathrm{~kg} \mathrm{BW}=8 \mathrm{mg} / \mathrm{kg} / \mathrm{d}
$$

Comments: No adverse effects were observed at either dose level. Therefore because the study considered exposure over 2 generations and included reproduction, the 100 ppm dose was considered to be a chronic NOAEL.

Final NOAEL: $8 \mathrm{mg} / \mathrm{kg} / \mathrm{d}$

Compound: $\quad$ 1,1,1-Trichloroethane

Form: not applicable

Reference: $\quad$ Lane et al. 1982

Test Species: Mouse

Body weight: $0.035 \mathrm{~kg}$ (from study)

Water Consumption: $6 \mathrm{~mL} / \mathrm{d}$ (from study)

Exposure Duration: 2 generations $(>1 \mathrm{yr}$ and during a critical lifestage $=$ chronic).

Endpoint:

Exposure Route:

reproduction

Dosage:

oral in water

three dose levels:

100,300 , and $1000 \mathrm{mg} / \mathrm{kg} / \mathrm{d}$

Calculations:

No effects observed at any dose level.

Comments: Because no significant differences were observed at any dose level and the study considered exposure throughout 2 generations including critical lifestages (reproduction), the maximum dose was considered to be a chronic NOAEL.

Final NOAEL: $\quad 1000 \mathrm{mg} / \mathrm{kg} / \mathrm{d}$.

Compound: Trichloroethylene

Form: not applicable

Reference: Buben and O'Flaherty 1985

Test Species: Mouse 
Body weight: $0.03 \mathrm{~kg}$ (EPA 1988a)

Exposure Duration: 6 weeks

Endpoint:

( $<1 \mathrm{yr}$ and not during a critical lifestage $=$ subchronic $)$.

Exposure Route:

Dosage:

Hepatotoxicity

oral gavage

seven dose levels (administered daily 5 days/week for 6 weeks): $100,200,400,800,1600,2400$, and $3200 \mathrm{mg} / \mathrm{kg} / \mathrm{d}$;

LOAEL $=100 \mathrm{mg} / \mathrm{kg} / \mathrm{d}$

Calculations:

not applicable

Comments: Because mice were exposed for 5 days/week, 7 day/week exposures were estimated by multiplying doses by 0.7 ( 5 days $/ 7$ days). Hepatotoxicity was observed at doses of $100 \mathrm{mg} / \mathrm{kg} / \mathrm{d}$ or greater. Therefore, the $100 \mathrm{mg} / \mathrm{kg} / \mathrm{d}$ dose was considered to be a subchronic LOAEL. A chronic NOAEL was estimated by multiplying the subchronic NOAEL by a subchronic-chronic uncertainty factor of 0.1 and a LOAEL-NOAEL uncertainty factor of 0.1 .

Final NOAEL: $0.7 \mathrm{mg} / \mathrm{kg} / \mathrm{d}$

Compound: Uranium

Form: $\quad$ Uranyl acetate $(61.32 \% \mathrm{U})$

Reference: Paternain et al. 1989

Test Species: Mouse

Body weight (from study): $0.028 \mathrm{~kg}$

Exposure Duration: $60 \mathrm{~d}$ prior to gestation, plus through gestation, delivery and lactation (during a critical lifestage $=$ chronic).

Endpoint: reproduction

Exposure Route:

Dosage: oral intubation three dose levels:

Calculations: 5,10 , and $25 \mathrm{mg}$ uranyl acetate $/ \mathrm{kg} / \mathrm{d} ; \mathrm{NOAEL}=5 \mathrm{mg} / \mathrm{kg} / \mathrm{d}$ or NOAEL dosage of elemental $U$ is:

$0.6132 \times 5 \mathrm{mg}$ uranyl acetate $/ \mathrm{kg} / \mathrm{d}$ or $3.07 \mathrm{mg} \mathrm{U} / \mathrm{kg} / \mathrm{d}$.

Comments: Significant differences in reproductive parameters (e.g., no.dead young/litter, size and weight of offspring, etc.) were observed at the 10 and $25 \mathrm{mg} / \mathrm{kg} / \mathrm{d}$ dose levels. Because no significant differences were observed at the $5 \mathrm{mg} / \mathrm{kg} / \mathrm{d}$ level and the study considered exposure throughout a critical lifestage (reproduction), this dose was considered to be a chronic NOAEL.

Final NOAEL: $\quad 3.07 \mathrm{mg} \mathrm{U} / \mathrm{kg} / \mathrm{d}$.

Compound: Uranium

Form: depleted metallic

Reference: Haseltine and Sileo 1983

Test Species: $\quad$ Black Duck

Body weight: $1.25 \mathrm{~kg}$ (mean $_{\delta+q}$; Dunning 1984) 
Food Consumption: Congeneric Mallard ducks, weighing $1 \mathrm{~kg}$ consume $100 \mathrm{~g}$ food/d (Heinz et al.1989). Therefore, it was assumed that a $1.25 \mathrm{~kg}$ black duck would consume $125 \mathrm{~g}$ food/d.

\section{Exposure Duration: 6 weeks}

( $<10$ wks and not during a critical lifestage $=$ subchronic $)$.

Endpoint: mortality, body weight, blood chemistry, liver or kidney effects

Exposure Route:

Dosage: oral in diet four dose levels: $25,100,400$, and $1600 \mathrm{ppm} \mathrm{U}$ in food;

Calculations: NOAEL $=1600 \mathrm{ppm}$

$$
\left(\frac{1600 \mathrm{mg} \mathrm{U}}{\mathrm{kg} \mathrm{food}} \times \frac{125 \mathrm{~g} \text { food }}{\text { day }} \times \frac{1 \mathrm{~kg}}{1000 \mathrm{~g}}\right) / 1.25 \mathrm{~kg} \mathrm{BW}=160 \mathrm{mg} / \mathrm{kg} / \mathrm{d}
$$

Comments: No effects observed at any dose level. Because this study was less than 10 weeks in duration and did not consider a critical lifestage (i.e., reproduction), it is considered to be subchronic. To estimate the chronic NOAEL, the subchronic NOAEL was multiplied by a subchronic-chronic uncertainty factor of 0.1 .

Final NOAEL: $\quad 16 \mathrm{mg} \mathrm{U} / \mathrm{kg} / \mathrm{d}$.

Compound: Vanadium

Form: $\quad$ Sodium Metavanadate $\left(\mathrm{NaVO}_{3} ; 41.78 \% \mathrm{~V}\right)$

Reference: $\quad$ Domingo et al. 1986

Test Species: Rat

Body weight (from study): $0.26 \mathrm{~kg}$

Exposure Duration: $60 \mathrm{~d}$ prior to gestation, plus through gestation, delivery and

Endpoint: lactation (during a critical lifestage $=$ chronic).

Exposure Route:

Dosage:

Calculations: reproduction

oral intubation

three dose levels:

5,10 , and $20 \mathrm{mg} \mathrm{NaVO} / \mathrm{kg} / \mathrm{d} ; \mathrm{LOAEL}=5 \mathrm{mg} / \mathrm{kg} / \mathrm{d}$

LOAEL dosage of elemental $V$ is:

$0.4178 \times 5 \mathrm{mg} \mathrm{NaVO} / 3 / \mathrm{kg} / \mathrm{d}$ or $2.1 \mathrm{mg} \mathrm{V} / \mathrm{kg} / \mathrm{d}$.

Comments: Significant differences in reproductive parameters (e.g., no.dead young/litter, size and weight of offspring, etc.) were observed at all dose levels. Therefore, the lowest dose was considered to be a chronic LOAEL. To estimate the chronic NOAEL, the chronic LOAEL was multiplied by a LOAEL-NOAEL uncertainty factor of 0.1 .

Final NOAEL: $\quad 0.21 \mathrm{mg} \mathrm{V} / \mathrm{kg} / \mathrm{d}$.

$\begin{array}{cl}\text { Compound: } & \text { Vanadium } \\ \text { Form: } & \text { Vanadyl Sulfate }\end{array}$


Reference: White and Dieter 1978

Test Species: $\quad$ Mallard Duck

Body weight: $1.17 \mathrm{~kg}$ (from study)

Food Consumption: $0.121 \mathrm{k} / \mathrm{d}$ (from study)

Exposure Duration: 12 weeks ( $>10$ wks $=$ chronic).

Endpoint:

Exposure Route:

Dosage:

mortality, body weight, blood chemistry

oral in diet

three dose levels:

$2.84,10.36$, and $110 \mathrm{ppm} \mathrm{V}$ in food;

Calculations:

NOAEL $=110 \mathrm{ppm}$

$$
\left(\frac{110 \mathrm{mg} \mathrm{V}}{\mathrm{kg} \text { food }} \times \frac{121 \mathrm{~g} \text { food }}{\text { day }} \times \frac{1 \mathrm{~kg}}{1000 \mathrm{~g}}\right) / 1.17 \mathrm{~kg} \mathrm{BW}=11.38 \mathrm{mg} / \mathrm{kg} / \mathrm{d}
$$

Comments: No effects observed at any dose level. Because this study was greater than 10 weeks in duration and did not consider a critical lifestage (i.e., reproduction), the maximum dose was considered to be a chronic NOAEL.

Final NOAEL: $\quad 11.38 \mathrm{mg} \mathrm{V} / \mathrm{kg} / \mathrm{d}$.

Compound: $\quad$ Vinyl Chloride

Form: not applicable

Reference: $\quad$ Feron et al. 1981

Test Species: Rat

Body weight: $0.35 \mathrm{~kg}$ (EPA 1988a)

Exposure Duration: lifetime ( $\sim 144$ wks)

Endpoint: longevity, mortality

Exposure Route: oral in diet

Dosage:

Calculations: three dose levels:

$1.7,5.0$, and $14.1 \mathrm{mg} / \mathrm{kg} / \mathrm{d} ; \mathrm{LOAEL}=1.7 \mathrm{mg} / \mathrm{kg} / \mathrm{d}$ or not applicable

Comments: Significantly reduced survivorship was observed at all dose levels, therefore the $1.7 \mathrm{mg} / \mathrm{kg} / \mathrm{d}$ dose level was considered to be a chronic LOAEL. To estimate the chronic NOAEL, the LOAEL was multiplied by a LOAEL-NOAEL uncertainty factor of 0.1 .

Final NOAEL: $\quad 0.17 \mathrm{mg} / \mathrm{kg} / \mathrm{d}$.

Compound: $\quad$ Xylene (mixed isomers)

Form: not applicable

Reference: $\quad$ Marks et al. 1982

Test Species: Mouse

Body weight: $0.03 \mathrm{~kg}$ (EPA 1988a) 
Exposure Duration: days 6-15 of gestation

Endpoint:

(during a critical lifestage $=$ chronic).

Exposure Route:

reproduction

Dosage:

oral gavage

six dose levels:

$0.52,1.03,2.06,2.58,3.10$, and $4.13 \mathrm{mg} / \mathrm{kg} / \mathrm{d}$;

NOAEL $=2.06 \mathrm{mg} / \mathrm{kg} / \mathrm{d}$

Calculations: not applicable

Comments: Xylene exposure of $2.58 \mathrm{mg} / \mathrm{kg} / \mathrm{d}$ or greater significantly reduced fetal weights and increased the incidence of fetal malformities. While the xylene exposures evaluated in this study were of a short duration, they occurred during a critical lifestage. Therefore, the highest dose that produced no adverse effects, $2.06 \mathrm{mg} / \mathrm{kg} / \mathrm{d}$, was considered to be a chronic NOAEL.

Final NOAEL: $2.06 \mathrm{mg} / \mathrm{kg} / \mathrm{d}$

$\begin{array}{lll}\text { Compound: } & \text { Zinc } \\ \text { Form: } & \text { Zinc Oxide } \\ \text { Reference: } & \text { Schlicker and Cox } 1968 \\ \text { Test Species: } & \text { Rat }\end{array}$

Body weight: $0.35 \mathrm{~kg}$ (EPA 1988a)

Food Consumption: $0.028 \mathrm{~kg} / \mathrm{d}$ (calculated using allometric equation from EPA 1988a)

Exposure Duration: days $1-16$ of gestation (during a critical lifestage $=$ chronic).

Endpoint:

Exposure Route: reproduction

Dosage: oral in diet two dose levels:

2000 , and $4000 \mathrm{ppm} \mathrm{Zn} ;$ NOAEL $=2000 \mathrm{ppm}$

Calculations:

$$
\left(\frac{2000 \mathrm{mg} \mathrm{Zn}}{\mathrm{kg} \mathrm{food}} \times \frac{28 \mathrm{~g} \text { food }}{\text { day }} \times \frac{1 \mathrm{~kg}}{1000 \mathrm{~g}}\right) / 0.35 \mathrm{~kg} \mathrm{BW}=160 \mathrm{mg} / \mathrm{kg} / \mathrm{d}
$$

Comments: Rats exposed to $4000 \mathrm{ppm} \mathrm{Zn}$ in the diet displayed increased rates of fetal resorption and reduced fetal growth rates. Because no effects were observed at the $2000 \mathrm{ppm} \mathrm{Zn}$ dose rate and the exposure occurred during gestation (a critical lifestage), this dose was considered a chronic NOAEL.

Final NOAEL: $160 \mathrm{mg} / \mathrm{kg} / \mathrm{d}$

$\begin{array}{ll}\text { Compound: } & \text { Zinc } \\ \text { Form: } & \text { Zinc Carbonate } \\ \text { Reference: } & \text { Gasaway and Buss } 1972\end{array}$


Test Species: $\quad$ Mallard Duck

Body Weight: $1 \mathrm{~kg}$ (from Heinz et al. 1989)

Food Consumption: $100 \mathrm{~g} / \mathrm{d}$ (from Heinz et al. 1989)

Exposure Duration: 60 days ( $<10 \mathrm{wks}$ and not during critical lifestage=subchronic)

Endpoint:

Exposure Route:

Dosage:

Mortality, body weight, and blood chemistry

oral in diet

four dose levels:

Calculations:

$3000,6000,9000$, and $12000 \mathrm{ppm} \mathrm{Zn} ; 3000 \mathrm{ppm}=$ LOAEL

$$
\left(\frac{3000 \mathrm{mg} \mathrm{Zn}}{\mathrm{kg} \text { food }} \times \frac{100 \mathrm{~g} \text { food }}{\text { day }} \times \frac{1 \mathrm{~kg}}{1000 \mathrm{mg}}\right) / 1 \mathrm{~kg} \mathrm{BW}=300 \mathrm{mg} / \mathrm{kg} / \mathrm{d}
$$

Comments: Because high mortality was observed at all does levels and the study was less than 10 weeks in duration, the lowest dose (3000 ppm $\mathrm{Zn})$ was considered a subchronic LOAEL. A chronic NOAEL was estimated by multiplying the subchronic LOAEL by a subchronic-chronic uncertainty factor of 0.1 and a LOAEL-NOAEL uncertainty factor of 0.1 .

Final NOAEL: $3 \mathrm{mg} / \mathrm{kg} / \mathrm{d}$

Compound: $\quad$ Zirconium

Form: Zirconium Sulfate

Reference: Schroeder et al. 1968b

Test Species: Mouse

Body weight: $0.03 \mathrm{~kg}$ (EPA 1988a)

Water Consumption: $0.0075 \mathrm{~L} / \mathrm{d}$

Food Consumption: $0.0055 \mathrm{~kg} / \mathrm{d}$

(calculated using allometric equation from EPA 1988a)

Exposure Duration: lifetime ( $>1 \mathrm{yr}=$ chronic).

Endpoint: lifespan, longevity

Exposure Route: oral in water (+incidental in food)

Dosage: one dose level:

Calculations:

$5 \mathrm{ppm} \mathrm{Zr}$ (in water) $+2.66 \mathrm{ppm} \mathrm{Zr}$ (in food) $=$ LOAEL

$\left(\frac{5 \mathrm{mg} \mathrm{Zr}}{L \text { water }} \times \frac{7.5 \mathrm{~mL} \text { water }}{\text { day }} \times \frac{1 \mathrm{~L}}{1000 \mathrm{~mL}}\right) / 0.03 \mathrm{~kg} \mathrm{BW}=1.25 \mathrm{mg} / \mathrm{kg} / \mathrm{d}$
$\left(\frac{2.66 \mathrm{mg} \mathrm{Zr}}{\mathrm{kg} \mathrm{food}} \times \frac{5.5 \mathrm{~g} \mathrm{food}}{\text { day }} \times \frac{1 \mathrm{~kg}}{1000 \mathrm{~g}}\right) / 0.03 \mathrm{~kg} \mathrm{BW}=0.488 \mathrm{mg} / \mathrm{kg} / \mathrm{d}$

Total Exposure $=1.25 \mathrm{mg} / \mathrm{kg} / \mathrm{d}+0.488 \mathrm{mg} / \mathrm{kg} / \mathrm{d}=1.738 \mathrm{mg} / \mathrm{kg} / \mathrm{d}$ 
Comments: Because no significant treatment effects were observed at the $5 \mathrm{ppm}$ dose level and the study considered exposure throughout the entire lifespan, this dose was considered to be a chronic NOAEL.

Final NOAEL: $1.738 \mathrm{mg} / \mathrm{kg} / \mathrm{d}$ 


\author{
APPENDIX B \\ Body Weights, Food and Water Consumptions \\ for Selected Avian and Mammalian Wildlife Endpoint Species
}


THIS PAGE INTENTIONALLY LEFT BLANK 
B-3

\begin{tabular}{|c|c|c|c|c|c|c|}
\hline \multirow[t]{2}{*}{ Species } & \multicolumn{2}{|r|}{ Body Weight } & \multicolumn{2}{|r|}{ Food Intake } & \multicolumn{2}{|r|}{ Water Intake } \\
\hline & kg & Citation & $\mathrm{kg} / \mathrm{d}$ & Citation & $L / d$ & Citation \\
\hline \multicolumn{7}{|l|}{ Mammals } \\
\hline $\begin{array}{l}\text { Short-tailed Shrew } \\
\text { (Blarina brevicauda) }\end{array}$ & 0.015 & Schlesinger and Potter 1974 & 0.009 & $\begin{array}{l}\text { Barrett and Stueck } 1976 \\
\text { Buckner } 1964\end{array}$ & 0.0033 & Chew 1951 \\
\hline $\begin{array}{l}\text { Little Brown Bat } \\
\text { (Myotis lucifugus) }\end{array}$ & 0.0075 & Gould 1955 & 0.0025 & Anthony and Kunz 1977 & 0.0012 & \\
\hline $\begin{array}{l}\text { Meadow Vole } \\
\text { (Microtus pennsylvanicus) }\end{array}$ & 0.044 & Reich 1981 & 0.005 & $\begin{array}{l}\text { Estimated from Figure 2. in } \\
\text { Dark et al. } 1983 .\end{array}$ & 0.006 & \\
\hline $\begin{array}{l}\text { White-footed Mouse } \\
\text { (Peromyscus leucopus) }\end{array}$ & 0.022 & Green and Miller 1987 & 0.0034 & Green and Miller 1987 & 0.0066 & Oswald et al. 1993 \\
\hline $\begin{array}{l}\text { Eastern Cottontail } \\
\text { (Sylvilagus floridanus) }\end{array}$ & 1.2 & Chapman et al. 1980 & 0.237 & Dalke and Sime 1941 & 0.116 & \\
\hline $\begin{array}{l}\text { Mink } \\
\text { (Mustela vison) }\end{array}$ & 1.0 & EPA $1993 \mathrm{e}$ & 0.137 & Bleavins and Aulerich 1981. & 0.099 & \\
\hline $\begin{array}{l}\text { Red Fox } \\
\text { (Vulpes fulva) }\end{array}$ & 4.5 & Storm et al. $1976^{b}$ & 0.45 & $\begin{array}{l}\text { Sargent } 1978^{\circ} \\
\text { Vogtsberger and Barrett } 1973\end{array}$ & 0.38 & \\
\hline $\begin{array}{l}\text { White-tailed Deer } \\
\text { (Odocoileus virginianus) }\end{array}$ & 56.5 & Smith 1991 & 1.74 & Mautz et al. 1976 & 3.7 & \\
\hline \multicolumn{7}{|l|}{ Birds } \\
\hline $\begin{array}{l}\text { American Robin } \\
\text { (Turdus migratorius) }\end{array}$ & 0.077 & Dunning 1984 & 0.093 & $\begin{array}{l}\text { Skorupa and Hothem } 1985 \\
\text { Hazelton et al. } 1984\end{array}$ & 0.0106 & \\
\hline $\begin{array}{l}\text { American Woodcock } \\
\text { (Scolopax minor) }\end{array}$ & 0.198 & Dunning 1984 & 0.15 & Sheldon 1975 & 0.02 & \\
\hline
\end{tabular}


Appendix B. Body Weights, Food and Water Consumption Rates, for Selected Avian and Mammalian Wildlife Endpoint Species

\begin{tabular}{|c|c|c|c|c|c|c|}
\hline \multirow[t]{2}{*}{ Species } & \multicolumn{2}{|r|}{ Body Weight } & \multicolumn{2}{|r|}{ Food Intake } & \multicolumn{2}{|c|}{ Water Intake } \\
\hline & kg & Citation & $\mathrm{kg} / \mathrm{d}$ & Citation & $L / d$ & Citation \\
\hline $\begin{array}{l}\text { Wild Turkey } \\
\text { (Meleagris gallipavo) }\end{array}$ & 5.8 & Dunning 1984 & 0.174 & Korschgen 1967 & 0.19 & \\
\hline $\begin{array}{l}\text { Belted Kingfisher } \\
\text { (Ceryle alcyon) }\end{array}$ & 0.148 & Dunning 1984 & 0.075 & Alexander 1977 & 0.016 & \\
\hline $\begin{array}{l}\text { Great Blue Heron } \\
\text { (Ardea herodias) }\end{array}$ & 2.39 & Dunning 1984 & 0.42 & Kushlan 1978 & 0.1058 & \\
\hline $\begin{array}{l}\text { Barred Owl } \\
\text { (Strix varia) }\end{array}$ & 0.717 & Dunning 1984 & 0.0468 & $\begin{array}{l}\text { Estimated according to } \\
\text { Nagy (1987) }\end{array}$ & 0.047 & \\
\hline $\begin{array}{l}\text { Barn Owl } \\
\text { (Tyto alba) }\end{array}$ & 0.466 & Johnsgard 1988 & 0.0625 & Johnsgard 1988 & 0.035 & \\
\hline $\begin{array}{l}\text { Cooper's Hawk } \\
\text { (Accipiter cooperi) }\end{array}$ & 0.439 & Dunning 1984 & 0.034 & $\begin{array}{l}\text { Estimated according to } \\
\text { Nagy (1987) }\end{array}$ & 0.034 & \\
\hline $\begin{array}{l}\text { Red-tailed Hawk } \\
\text { (Buteo jamaciencis) }\end{array}$ & 1.126 & Dunning 1984 & 0.91 & Wakely 1978 & 0.064 & \\
\hline
\end{tabular}

"All values calculated according to Calder and Braun (1983) unless otherwise stated.

${ }^{b}$ Mean for males and females from both Iowa and Illinois.

c $0.069 \mathrm{~g} / \mathrm{g} / \mathrm{d}$ for nonbreeding adult times $4.5 \mathrm{~kg} \mathrm{BW}$ 


\section{APPENDIX C}

Selected Toxicity Data for Avian and Mammalian Wildlife 
THIS PAGE INTENTIONALLY LEFT BLANK 


\section{C-3}

\begin{tabular}{|c|c|c|c|c|c|c|}
\hline \multirow[b]{2}{*}{ Chemical } & \multirow[b]{2}{*}{ Species } & \multicolumn{2}{|c|}{ LOAEL } & \multirow{2}{*}{$\frac{\text { NOAEL }}{\text { Dose or Conc. }^{b}}$} & \multirow{2}{*}{$\begin{array}{l}\text { Acute or } \\
\text { Lethal } \\
\text { Dose/Conc. }\end{array}$} & \multirow{2}{*}{$\begin{array}{c}\mathrm{LD}_{50} \text { or } \\
\mathrm{LC}_{50}\end{array}$} \\
\hline & & Dose or Conc. ${ }^{b}$ & Effect & & & \\
\hline Aroclor 1016 & ferret & & & $20 \mathrm{ppm}(9 \mathrm{mo})$ & & \\
\hline Aroclor 1016 & $\operatorname{mink}$ & $20 \mathrm{ppm}(9 \mathrm{mo})$ & reproduction & & $20 \mathrm{ppm}$ & \\
\hline Aroclor 1221 & bobwhite quail & & $30 \%$ mortality & & $6000 \mathrm{ppm}(5 \mathrm{~d})$ & \\
\hline Aroclor 1221 & Japanese quail & & & & & $>6000 \mathrm{ppm}(5 \mathrm{~d})$ \\
\hline Aroclor 1221 & $\begin{array}{l}\text { ring-necked } \\
\text { pheasant }\end{array}$ & & & & $\begin{array}{c}>4000 \mathrm{ppm} \\
(5 \mathrm{~d})\end{array}$ & \\
\hline Aroclor 1232 & bobwhite quail & & & & & $3002 \mathrm{ppm}(5 \mathrm{~d})$ \\
\hline Aroclor 1232 & Japanese quail & & & & & $>5000 \mathrm{ppm}(5 \mathrm{~d})$ \\
\hline Aroclor 1232 & $\begin{array}{l}\text { ring-necked } \\
\text { pheasant }\end{array}$ & & & & & $3146 \mathrm{ppm}(5 \mathrm{~d})$ \\
\hline Arocior 1242 & ferret & $20 \mathrm{ppm}(9 \mathrm{mo})$ & reproduction & & $20 \mathrm{ppm}$ & \\
\hline Aroclor 1242 & $\operatorname{mink}$ & 5 ppm (9 mo) & reproduction & & $\begin{array}{r}10 \mathrm{ppm} \\
(9 \mathrm{mo}) \\
\end{array}$ & \\
\hline Aroclor 1242 & Japanese quail & $\begin{array}{l}321.5 \mathrm{ppm} \\
(21 \mathrm{~d})\end{array}$ & reproduction & & & \\
\hline Aroclor 1242 & Japanese quail & $10 \mathrm{ppm}(45 \mathrm{~d})$ & reproduction & & & \\
\hline Aroclor 1248 & screech owl & & reproduction & 3 ppm (18 mo) & & \\
\hline Aroclor 1248 & chicken & $10 \mathrm{ppm}(8 \mathrm{wk})$ & reproduction & $1 \mathrm{ppm}(8 \mathrm{wk})$ & & \\
\hline Aroclor 1254 & raccoon & $50 \mathrm{mg} / \mathrm{kg}(8 \mathrm{~d})$ & physiology & & & \\
\hline Aroclor 1254 & cottontail rabbit & $10 \mathrm{ppm}(12 \mathrm{wk})$ & weight loss & & & \\
\hline
\end{tabular}


C-4

\begin{tabular}{|c|c|c|c|c|c|c|}
\hline \multirow[b]{2}{*}{ Chemical } & \multirow[b]{2}{*}{ Species } & \multicolumn{2}{|c|}{ LOAEL } & \multirow{2}{*}{ NOAEL } & \multirow{2}{*}{$\begin{array}{c}\text { Acute or } \\
\text { Lethal } \\
\text { Dose/Conc. }\end{array}$} & \multirow{2}{*}{$\begin{array}{l}L D_{50} \text { or } \\
L C_{50}\end{array}$} \\
\hline & & Dose or Conc. & Effect & & & \\
\hline Aroclor 1254 & $\begin{array}{l}\text { white-footed } \\
\text { mouse }\end{array}$ & $10 \mathrm{ppm}(18 \mathrm{mo})$ & $\begin{array}{l}\text { reproduction; } \\
\text { decreased pup } \\
\text { survival }\end{array}$ & & & \\
\hline Aroclor 1254 & quail & $50 \mathrm{ppm}$ (14 wk) & reproduction & & & \\
\hline Aroclor 1254 & Japanese quail & $78.1 \mathrm{ppm}(21 \mathrm{~d})$ & reproduction & & & \\
\hline Aroclor 1254 & Japanese quail & & & $20 \mathrm{ppm}(8 \mathrm{wk})$ & & \\
\hline Aroclor 1254 & Japanese quail & $5 \mathrm{ppm}(12 \mathrm{wk})$ & physiology & & & \\
\hline Aroclor 1254 & mourning dove & $40 \mathrm{ppm}(42 \mathrm{~d})$ & metabolism & & & \\
\hline Aroclor 1254 & ring dove & $10 \mathrm{ppm}$ & reproduction & & & \\
\hline Aroclor 1254 & pheasant & $\begin{array}{l}12.5 \mathrm{mg} \\
(1 \mathrm{x} / \mathrm{wk}, 17 \mathrm{wk})\end{array}$ & & & & \\
\hline Aroclor 1260 & bobwhite quail & $5 \mathrm{ppm}(4 \mathrm{mo})$ & thyroid weight & & & \\
\hline Aroclor 1260 & Japanese quail & $62.5 \mathrm{ppm}(21 \mathrm{~d})$ & reproduction & & & \\
\hline Arsanilic acid & rat & & & & & $216 \mathrm{mg} / \mathrm{kg}$ \\
\hline Cadmium & deer mouse & $1 \mathrm{mg} / \mathrm{L}$ & infertility & & & \\
\hline Cadmium & wood duck & $100 \mathrm{ppm}(3 \mathrm{mo})$ & pathology & $10 \mathrm{ppm}(3 \mathrm{mo})$ & & \\
\hline Cadmium & black duck & $4 \mathrm{ppm}(4 \mathrm{mo})$ & $\begin{array}{l}\text { offspring } \\
\text { behavior }\end{array}$ & & & \\
\hline Cadmium chloride & mallard duck & $\begin{array}{l}20 \mathrm{ppm} \\
(30-90 \mathrm{~d})\end{array}$ & pathology & & & \\
\hline Cadmium succinate & bobwhite quail & & & & & $1728 \mathrm{ppm}(5 \mathrm{~d})$ \\
\hline
\end{tabular}


C-5

\begin{tabular}{|c|c|c|c|c|c|c|}
\hline \multirow[b]{2}{*}{ Chemical } & \multirow[b]{2}{*}{ Species } & \multicolumn{2}{|c|}{ LOAEL } & \multirow{2}{*}{$\frac{\text { NOAEL }}{\text { Dose or Conc. }^{b}}$} & \multirow{2}{*}{$\begin{array}{c}\text { Acute or } \\
\text { Lethal } \\
\text { Dose/Conc. }\end{array}$} & \multirow{2}{*}{$\begin{array}{l}\mathrm{LD}_{30} \text { or } \\
\mathrm{LC}_{50}\end{array}$} \\
\hline & & Dose or Conc. ${ }^{b}$ & Effect & & & \\
\hline Cadmium succinate & Japanese quail & & & & & 2693 ppm (5 d) \\
\hline Cadmium succinate & $\begin{array}{l}\text { ring-necked } \\
\text { pheasant }\end{array}$ & & & & & 1411 ppm (5 d) \\
\hline Cadmium succinate & mallard duck & & & & & $>5000 \mathrm{ppm}(5 \mathrm{~d})$ \\
\hline Chlordane & bobwhite quail & & & & & 331 ppm (5 day) \\
\hline Chlordane & Japanese quail & & & & & $350 \mathrm{ppm}(5 \mathrm{~d})$ \\
\hline Chlordane & Japanese quail & $25 \mathrm{ppm}(8 \mathrm{~d})$ & reproduction & & & \\
\hline Chlordane & $\begin{array}{l}\text { ring-necked } \\
\text { pheasant }\end{array}$ & & & & & 430 ppm (5d) \\
\hline Chlordane & mallard duck & & & & & $858 \mathrm{ppm}(5 \mathrm{~d})$ \\
\hline Chlordane & golden eagle & & & & $100 \mathrm{mg} / \mathrm{kg}$ & $10 \mathrm{mg} / \mathrm{kg}$ \\
\hline Chromium (trivalent) & $\begin{array}{l}\text { black duck } \\
\text { (young) }\end{array}$ & $10 \mathrm{ppm}$ & survival & & & \\
\hline $\begin{array}{l}\text { Chromium - potassium } \\
\text { dichromate }\end{array}$ & Japanese quail & & $5-d L_{50}$ & & & $4400 \mathrm{ppm}$ \\
\hline $2,4, \mathrm{D}$ & deer mouse & & & $3 \mathrm{lb} / \mathrm{acre}$ & & \\
\hline DDD & cowbird & $1500 \mathrm{ppm}(17 \mathrm{~d})$ & lethal & & & \\
\hline DDE & cowbird & $1500 \mathrm{ppm}(27 \mathrm{~d})$ & lethal & & & \\
\hline DDE & Japanese quail & $25 \mathrm{ppm}(14 \mathrm{wk})$ & $\begin{array}{l}\text { reproduction; } \\
\text { liver }\end{array}$ & 5 ppm (12 wk) & & \\
\hline DDE & rat-tailed bat & & & 107 ppm (40 d) & & \\
\hline
\end{tabular}


C-6

\begin{tabular}{|c|c|c|c|c|c|c|}
\hline \multirow[b]{2}{*}{ Chemical } & \multirow[b]{2}{*}{ Species } & \multicolumn{2}{|c|}{ LOAEL } & \multirow{2}{*}{$\frac{\text { NOAEL }}{\text { Dose or Conc. }}$} & \multirow{2}{*}{$\begin{array}{l}\text { Acute or } \\
\text { Lethal } \\
\text { Dose/Conc. }\end{array}$} & \multirow{2}{*}{$\begin{array}{l}\mathrm{LD}_{50} \text { or } \\
\mathrm{LC}_{50}\end{array}$} \\
\hline & & Dose or Conc. & Effect & & & \\
\hline p,p'-DDE & mallard duck & $\begin{array}{l}5 \mathrm{ppm} \text { (several } \\
\text { mo) }\end{array}$ & thin egg shells & $1 \mathrm{ppm}$ & & \\
\hline p,p'-DDE & black duck & $\begin{array}{l}10 \mathrm{ppm}(6 \\
\mathrm{mo} / \mathrm{yr})\end{array}$ & thin egg shells & & & \\
\hline p,p'-DDE & pigeon & $18 \mathrm{mg} / \mathrm{kg}(8 \mathrm{wk})$ & & & $\begin{array}{c}36 \mathrm{mg} / \mathrm{kg} \\
(8 \mathrm{wk}) \\
\end{array}$ & \\
\hline DDT & Japanese quail & $25 \mathrm{ppm}$ (14 wk) & reproduction & & & \\
\hline DDT & Japanese quail & $50 \mathrm{ppm}(10 \mathrm{wk})$ & reproduction & $5 \mathrm{ppm}(10 \mathrm{wk})$ & & \\
\hline DDT & bobwhite quail & $500 \mathrm{ppm}(4 \mathrm{mo})$ & thyroid & $50 \mathrm{ppm}(4 \mathrm{mo})$ & & \\
\hline DDT & mallard duck & $330 \mathrm{ppm}(5 \mathrm{~d})$ & growth & & & \\
\hline DDT & mallard duck & $50 \mathrm{ppm}(6 \mathrm{mo})$ & & & & \\
\hline DDT & mallard duck & & & & & $1869 \mathrm{ppm}(5 \mathrm{~d})$ \\
\hline DDT & house sparrow & & & & $1500 \mathrm{ppm}(3 \mathrm{~d})$ & \\
\hline DDT & $\begin{array}{l}\text { white-throated } \\
\text { sparrow }\end{array}$ & 5 ppm (11 wk) & $\begin{array}{l}\text { behavior; } \\
\text { physiology }\end{array}$ & & & \\
\hline DDT & earthworm & $5 \mathrm{lb} / \mathrm{acre}$ & $\begin{array}{l}\text { decreased } \\
\text { population }\end{array}$ & & & \\
\hline Di-butyl phthalate & mallard duck & & $\begin{array}{l}\text { 5-d lethal } \\
\text { concentration }\end{array}$ & & $>5000 \mathrm{ppm}$ & \\
\hline Di-butyl phthalate & ring dove & $10 \mathrm{ppm}$ & thin egg shells & & & \\
\hline
\end{tabular}




\section{C-7}

\begin{tabular}{|c|c|c|c|c|c|c|}
\hline \multirow[b]{2}{*}{ Chemical } & \multirow[b]{2}{*}{ Species } & \multicolumn{2}{|c|}{ LOAEL } & \multirow{2}{*}{$\frac{\text { NOAEL }}{\text { Dose or Conc. }^{b}}$} & \multirow{2}{*}{$\begin{array}{c}\text { Acute or } \\
\text { Lethal } \\
\text { Dose/Conc. }\end{array}$} & \multirow{2}{*}{$\begin{array}{l}L^{L} D_{50} \text { or } \\
L C_{50}\end{array}$} \\
\hline & & Dose or Conc. ${ }^{b}$ & Effect & & & \\
\hline $\begin{array}{l}\text { 2,4-Dichlorophenyl-p- } \\
\text { nitrophenyl ether }\end{array}$ & rat & $100 \mathrm{ppm}(97 \mathrm{wk})$ & reproduction & 10 ppm (3 gen.) & & $2600 \mathrm{ppm}$ \\
\hline $\begin{array}{l}\text { 2,4-Dichlorophenyl-p- } \\
\text { nitrophenyl ether }\end{array}$ & $\operatorname{dog}$ & & & $2000 \mathrm{ppm}(2 \mathrm{yr})$ & & \\
\hline Di(2-ethylhexyl)phthalate & ferret & $\begin{array}{l}10000 \mathrm{ppm} \\
(14 \mathrm{mo})\end{array}$ & physiology & & & \\
\hline Di(2-ethylhexyl)phthalate & ring dove & & & $10 \mathrm{ppm}$ & & \\
\hline Ferrous sulfate & rat & & & & & $1187 \mathrm{mg} / \mathrm{kg}$ \\
\hline Hexachlorobenzene & Japanese quail & $20 \mathrm{ppm}(90 \mathrm{~d})$ & reproduction & & & \\
\hline Hexachlorobenzene & Japanese quail & & & & $\begin{array}{l}1 \mathrm{ppm} \\
(90 \mathrm{~d})\end{array}$ & \\
\hline Hexachlorobenzene & mallard duck & & $30 \%$ mortality & & $5000 \mathrm{ppm}$ & $>5000 \mathrm{ppm}$ \\
\hline Hexachlorobutadiene & Japanese quail & $0.3 \mathrm{ppm}(90 \mathrm{~d})$ & & & & \\
\hline Hexachlorophene & rat & 100 ppm (3 gen.) & reproduction & 20 ppm (3 gen.) & & \\
\hline $\begin{array}{l}\text { Hexamethylphosphoric } \\
\text { triamide }\end{array}$ & rat & $\begin{array}{l}2 \mathrm{mg} / \mathrm{kg} / \mathrm{d} \\
(169 \mathrm{~d})\end{array}$ & reproduction & & & \\
\hline Kepone & Japanese quail & & & & $\begin{array}{r}200 \mathrm{ppm} \\
(240 \mathrm{~d}) \\
\end{array}$ & \\
\hline Lead & bobwhite quail & & & $\begin{array}{l}2000 \mathrm{ppm}(6 \\
\mathrm{wk})\end{array}$ & & \\
\hline Lead acetate & Japanese quail & $1 \mathrm{ppm}(12 \mathrm{wk})$ & reproductiion & & & \\
\hline
\end{tabular}


C-8

\begin{tabular}{|c|c|c|c|c|c|c|}
\hline \multirow[b]{3}{*}{ Chemical } & \multicolumn{6}{|c|}{ Appendix C. Selected Toxicity Data for Avian and Mammalian Wildlife } \\
\hline & \multirow[b]{2}{*}{ Species } & \multicolumn{2}{|c|}{ LOAEL } & \multirow{2}{*}{$\frac{\text { NOAEL }}{\text { Dose or Conc. }}$} & \multirow{2}{*}{$\begin{array}{l}\text { Acute or } \\
\text { Lethal } \\
\text { Dose/Conc. }\end{array}$} & \multirow{2}{*}{$\begin{array}{l}\mathrm{LD}_{50} \text { or } \\
\mathrm{LC}_{50}\end{array}$} \\
\hline & & Dose or Conc. ${ }^{b}$ & Effect & & & \\
\hline Lead acetate & bobwhite quail & $1000 \mathrm{ppm}(6 \mathrm{wk})$ & growth & & & \\
\hline Lead arsenate & rat & & & & & $1545 \mathrm{mg} / \mathrm{kg}$ \\
\hline Lead arsonate & Japanese quail & & & & & $4185 \mathrm{ppm}(5 \mathrm{~d})$ \\
\hline Lead arsonate & $\begin{array}{l}\text { ring-necked } \\
\text { pheasant }\end{array}$ & & & & & 4989 ppm (5 d) \\
\hline Lead, tetraethyl & mallard duck & & & & $6 \mathrm{mg} / \mathrm{kg}$ & \\
\hline Lithium chloride & $\begin{array}{l}\text { red-winged } \\
\text { blackbird }\end{array}$ & & & & $\begin{array}{c}15000 \mathrm{ppm} \\
(4 \mathrm{~d})\end{array}$ & \\
\hline Magnesium & Japanese quail & $\begin{array}{l}1500 \mathrm{ppm} \\
(2 \mathrm{wk})\end{array}$ & physiology & $\begin{array}{l}1000 \mathrm{ppm} \\
(2 \mathrm{wk})\end{array}$ & & \\
\hline Mercuric chloride & Japanese quail & & & $2 \mathrm{ppm}(1 \mathrm{yr})$ & & \\
\hline Mercuric chloride & Japanese quail & $4 \mathrm{ppm}(12 \mathrm{wk})$ & physiology & $2 \mathrm{ppm}$ & & \\
\hline Mercuric chloride & chicken & 100 ppm (8 wk) & reproduction & & & \\
\hline Mercuric sulfate & chicken & $100 \mathrm{ppm}(8 \mathrm{wk})$ & reproduction & & & \\
\hline Methyl mercury chloride & mallard duck & & & $5 \mathrm{ppm}(3 \mathrm{mo})$ & & \\
\hline Methyl mercury chloride & chicken & $5 \mathrm{ppm}(8 \mathrm{wk})$ & reproduction & & & \\
\hline $\begin{array}{l}\text { Methyl mercury } \\
\text { dicyandiamide }\end{array}$ & mallard duck & $0.5 \mathrm{ppm}(1 \mathrm{yr})$ & reproduction & & & \\
\hline $\begin{array}{l}\text { Methyl mercury } \\
\text { dicyandiamide }\end{array}$ & black duck & $\begin{array}{l}3 \mathrm{ppm} \\
(28 \mathrm{wk} / \mathrm{yr}, 2 \mathrm{yr})\end{array}$ & reproduction & & & \\
\hline
\end{tabular}


C-9

\begin{tabular}{|c|c|c|c|c|c|c|}
\hline \multirow[b]{2}{*}{ Chemical } & \multirow[b]{2}{*}{ Species } & \multicolumn{2}{|c|}{ LOAEL } & \multirow{2}{*}{$\frac{\text { NOAEL }}{\text { Dose or Conc. }}$} & \multirow{2}{*}{$\begin{array}{l}\text { Acute or } \\
\text { Lethal } \\
\text { Dose/Conc. }\end{array}$} & \multirow{2}{*}{$\begin{array}{l}\mathrm{LD}_{50} \text { or } \\
\mathrm{LC}_{50}\end{array}$} \\
\hline & & Dose or Conc. ${ }^{b}$ & Effect & & & \\
\hline $\begin{array}{l}\text { Monosodium } \\
\text { methanearsonate }\end{array}$ & $\begin{array}{l}\text { white-footed } \\
\text { mouse }\end{array}$ & $1000 \mathrm{ppm}(30 \mathrm{~d})$ & physiology & & & $300 \mathrm{mg} / \mathrm{kg}$ \\
\hline $\begin{array}{l}\text { Octochlorodibenzo-p- } \\
\text { dioxin }\end{array}$ & rat & $\begin{array}{l}0.5 \mathrm{mg} / \mathrm{kg} \\
(2 \mathrm{wk})\end{array}$ & pathology & $\begin{array}{l}0.1 \mathrm{mg} / \mathrm{kg} \\
(2 \mathrm{wk})\end{array}$ & & \\
\hline $\begin{array}{l}\text { PBB } \\
\text { (hexabromo biphenyl) }\end{array}$ & Japanese quail & $100 \mathrm{ppm}(9 \mathrm{wk})$ & reproduction & $20 \mathrm{ppm}(9 \mathrm{wk})$ & & \\
\hline $\begin{array}{l}\text { PBB } \\
\text { (polybrominated } \\
\text { biphenyl) }\end{array}$ & mink & $1 \mathrm{ppm}(10 \mathrm{mo})$ & reproduction & & & $\begin{array}{c}179 \mathrm{mg} / \mathrm{kg} \\
3.95 \mathrm{ppm}\end{array}$ \\
\hline PBB & Japanese quail & $25 \mathrm{ppm}(7 \mathrm{~d})$ & $\begin{array}{l}\text { blood } \\
\text { chemistry }\end{array}$ & & & \\
\hline Sodium arsenite & mallard duck & $100 \mathrm{mg} / \mathrm{kg}(1 \mathrm{~d})$ & thin eggshells & & & \\
\hline Sodium cyanide & coyote & $4 \mathrm{mg} / \mathrm{kg}$ & physiology & & & \\
\hline $\begin{array}{l}\text { Sodium } \\
\text { monofluoroacetate }\end{array}$ & mallard duck & & & & & $3.71 \mathrm{mg} / \mathrm{kg}$ \\
\hline $\begin{array}{l}\text { Sodium } \\
\text { monofluoroacetate }\end{array}$ & mallard duck & & & & $9.11 \mathrm{mg} / \mathrm{kg}$ & \\
\hline $\begin{array}{l}\text { Sodium } \\
\text { monofluoroacetate }\end{array}$ & $\begin{array}{l}\text { ring-necked } \\
\text { pheasant }\end{array}$ & & & & $6.46 \mathrm{mg} / \mathrm{kg}$ & \\
\hline $\begin{array}{l}\text { Sodium } \\
\text { monofluoroacetate }\end{array}$ & chukar partridge & & & & $3.51 \mathrm{mg} / \mathrm{kg}$ & \\
\hline $\begin{array}{l}\text { Sodium } \\
\text { monofluoroacetate }\end{array}$ & quail & & & & $17.7 \mathrm{mg} / \mathrm{kg}$ & \\
\hline
\end{tabular}


C-10

\begin{tabular}{|c|c|c|c|c|c|c|}
\hline \multirow[b]{2}{*}{ Chemical } & \multirow[b]{2}{*}{ Species } & \multicolumn{2}{|c|}{ LOAEL } & \multirow{2}{*}{$\frac{\text { NOAEL }}{\text { Dose or Conc. }^{6}}$} & \multirow{2}{*}{$\begin{array}{l}\text { Acute or } \\
\text { Lethal } \\
\text { Dose/Conc. }\end{array}$} & \multirow{2}{*}{$\begin{array}{l}L^{L D_{50}} \text { or } \\
L C_{50}\end{array}$} \\
\hline & & Dose or Conc. ${ }^{b}$ & Effect & & & \\
\hline $\begin{array}{l}\text { Sodium } \\
\text { monofluoroacetate }\end{array}$ & pigeon & & & & $4.24 \mathrm{mg} / \mathrm{kg}$ & \\
\hline $\begin{array}{l}\text { Sodium } \\
\text { monofluoroacetate }\end{array}$ & house sparrow & & & & $3.00 \mathrm{mg} / \mathrm{kg}$ & \\
\hline $\begin{array}{l}\text { Sodium } \\
\text { monofluoroacetate }\end{array}$ & kit fox & & & & & $0.22 \mathrm{mg} / \mathrm{kg}$ \\
\hline Sodium nitrate & Japanese quail & & & & $3300 \mathrm{ppm}(7 \mathrm{~d})$ & \\
\hline Sodium nitrate & Japanese quail & & & & $\begin{array}{c}660 \mathrm{ppm}(15 \\
\mathrm{wk})\end{array}$ & \\
\hline Thallium sulfate & golden eagle & & & & & $120 \mathrm{mg} / \mathrm{kg}$ \\
\hline Tribromoethanol & mallard duck & & & & $150 \mathrm{mg} / \mathrm{kg}$ & \\
\hline Vanadyl sulfate & mallard duck & $\begin{array}{r}100 \mathrm{ppm} \\
(12 \mathrm{wk}) \\
\end{array}$ & $\begin{array}{l}\text { blood } \\
\text { chemistry }\end{array}$ & 10 ppm (12 wk) & & \\
\hline Zinc phosphide & kit fox & & & & & $93 \mathrm{mg} / \mathrm{kg}$ \\
\hline Zinc phosphide & red fox & & & & $\begin{array}{c}10.64 \mathrm{mg} / \mathrm{kg} / \mathrm{d} \\
(3 \mathrm{~d})\end{array}$ & \\
\hline Zine phosphide & grey fox & & & & $\begin{array}{c}8.6 \mathrm{mg} / \mathrm{kg} / \mathrm{d} \\
(3 \mathrm{~d})\end{array}$ & \\
\hline Zinc phosphide & great horned owl & & & & $\begin{array}{c}22.31 \mathrm{mg} / \mathrm{kg} / \mathrm{d} \\
(3 \mathrm{~d})\end{array}$ & \\
\hline
\end{tabular}

- Data extracted from TERRE-TOX database (Meyers and Schiller 1986). Complete citations for these data are not currently available.

' Dose in $\mathrm{mg} / \mathrm{kg} /$ day; dietary concentration in $\mathrm{ppm}$; water concentration in $\mathrm{mg} / \mathrm{L}$. 


\section{DISTRIBUTION}

ES/ER/TM-86/R1

1. J. Archer

2. L. W. Barnthouse

3. L. Baron

4. B. G. Blaylock

5. R. R. Bonczek

6. M. Clauberg

7. J. Dee

8. J. R. Duncan

9. M. Ferré

10. D. Gonzales

11. R. N. Hull

12. D. S. Jones

13. R. C. Kramel

14. S. Lampkins

15. M. Leslie

16. R. Mathis

17-9. D. M. Matteo

20. C. W. McGinn

21. D. Mentzer

22. P. D. Miller

23. D. B. Miller
24. B. D. Nourse

25-6. P. T. Owen

27. S. Pack

28. S. T. Purucker

29. Sue Reith

30. B. E. Sample

31. D. M. Steinhauff

32. G. Stephens

33. G. W. Suter

34. Andrea Temeshy

35. C. C. Travis

36. C. J. E. Welch

37. R. K. White

38. Don Wilkes

39. E. Will

40. Central Research Library

41-2. ER Document Management Center

43. Laboratory Records

44. ORNL Patent Section

45. D. M. Opresko

46. Office of Assistant Manager for Energy Research and Development, DOE Oak Ridge Field Office, P.O. Box 2001, Oak Ridge, TN 37831-8600.

47. Office of Scientific and Technical Information (OSTI), P.O. Box 62, Oak Ridge, TN, 37831. 